\title{
UNDERSTANDING THE ROLE OF SHAFT STIFFNESS IN THE GOLF \\ SWING
}

\author{
A Thesis Submitted to the College of \\ Graduate Studies and Research in Partial \\ Fulfillment of the Requirements for the \\ Degree of Doctor of Philosophy in the \\ College of Kinesiology \\ University of Saskatchewan \\ Saskatoon, Saskatchewan
}

By

Sasho J. MacKenzie

(C) Copyright Sasho J. MacKenzie, December 2005. All rights reserved. 


\section{PERMISSION TO USE}

In presenting this thesis in partial fulfillment of the requirements for a Postgraduate degree from the University of Saskatchewan, I agree that the Libraries of the University may make it freely available for inspection. I further agree that permission for copying of this thesis in any manner, in whole or in part, for scholarly purposes may be granted by the professor or professors who supervised my thesis work or, in their absence, by the Head of the Department or the Dean of the College in which this thesis work was done. It is understood that any copying or publication or use of this thesis or parts thereof for financial gain shall not be allowed without my written permission. It is also understood that due recognition shall be given to me and the University of Saskatchewan in any scholarly use which may be made of any material in my thesis.

Requests for permission to copy or to make any other use of material in this thesis in whole or in part should be addressed to:

The Dean

College of Kinesiology

University of Saskatchewan

87 Campus Drive

Saskatoon, Saskatchewan, Canada, S7N 5B2 


\section{ACKNOWLEDGEMENT}

I would like to thank my supervisor, Dr. Eric Sprigings and my committee members, Dr. Gordon Binsted, Dr. Denise Stilling, and Dr. Glen Watson for their support and advice throughout the preparation and completion of this thesis. I would also like to thank Dr. Mont Hubbard for acting as the external examiner.

I would like to thank Dr. Graham Caldwell and Dr. Joseph Hamill for providing me with the opportunity to further my understanding of biomechanics in their research laboratory. Your welcome was warm, expertise invaluable, and guidance appreciated.

I would like to acknowledge the Natural Sciences and Engineering Research Council of Canada for providing funding support during the completion of this thesis. 


\begin{abstract}
The purpose of this thesis was to determine how shaft stiffness affects clubhead speed and how it alters clubhead orientation at impact. For the first time, a 3D, sixsegment forward dynamics model of a golfer and club was developed and optimized to answer these questions. A range of shaft stiffness levels from flexible to stiff were evaluated at three levels of swing speed (38, 45 and $53 \mathrm{~m} / \mathrm{s})$. At any level of swing speed, the difference in clubhead speed did not exceed $0.1 \mathrm{~m} / \mathrm{s}$ across levels of shaft stiffness. Therefore, it was concluded that customizing the stiffness of a golf club shaft to perfectly suit a particular swing will not increase clubhead speed sufficiently to have any meaningful effect on performance. The magnitude of lead deflection at impact increased as shaft stiffness decreased. The magnitude of lead deflection at impact also increased as swing speed increased. For an optimized swing that generated a clubhead speed of $45 \mathrm{~m} / \mathrm{s}$, with a shaft of regular stiffness, lead deflection of the shaft at impact was $6.25 \mathrm{~cm}$. The same simulation resulted in a toe-down shaft deflection of $2.27 \mathrm{~cm}$ at impact. Using the model, it was estimated that for each centimeter of lead deflection of the shaft, dynamic loft increased by approximately $0.8^{\circ}$. Toe-down shaft deflection had relatively no influence on dynamic loft. For every centimeter increase in lead deflection of the shaft, dynamic closing of the clubface increased by approximately $0.7^{\circ}$. For every centimeter increase in toe-down shaft deflection, dynamic closing of the clubface decreased by approximately $0.5^{\circ}$. The results from this thesis indicate that improvements in driving distance brought about by altering shaft stiffness are the result of altered clubhead orientation at impact and not increased clubhead speed.
\end{abstract}




\section{TABLE OF CONTENTS}

PERMISSION TO USE $\quad$ i

ACKNOWLEDGEMENT

ABSTRACT

TABLE OF CONTENTS iv

LIST OF TABLES viii

LIST OF FIGURES IX

CHAPTER 1 INTRODUCTION TO THE STUDY 1

$\begin{array}{lll}1.1 & \text { Introduction } & 2\end{array}$

1.2 Literature Review of Shaft Bending 4

1.2.1 Shaft Bending During the Swing 4

1.2.2 Utilization of Energy Stored in the Shaft 9

1.2.3 Variations in Force and Torque Patterns applied to the Club 14

1.2.4 Changes in Clubhead Orientation due to Shaft Deflection 16

1.2.5 Cause of Shaft Bending from a Newtonian Perspective 16

1.2.5.1 Tangential Force Component 17

1.2.5.2 Radial Force Component 18

1.2.6 Optimization of Shaft Behaviour 20

$\begin{array}{ll}\text { 1.3 Statement of Problem and Hypotheses } & 22\end{array}$

1.3.1 The Problem 22

1.3.2 Research Hypotheses 22

1.4 Review of Optimized Forward Dynamic Simulations 23 
1.4.1 Develop the Physical Properties of each Segment 25

1.4.2 Provide the Model with the Ability to Generate Motion 28

1.4.3 Develop Equations that will Govern the Motion of the Model 32

1.4.4 Determine How the Equations will be Solved 40

1.4.5 Implement an Optimization Scheme $\quad 42$

CHAPTER 2 METHODS 50

2.1 Mathematical Model 51

2.1.1 General Description of Golfer-Club Model 51

2.1.2 Method for Measuring Shaft Deflection 56

2.1.3 Determining Shaft Stiffness and Damping Parameters 57

$\begin{array}{lll}2.1 .4 & \text { Equations of Motion } & 61\end{array}$

2.1.5 Generalized Coordinates of the Golfer Model 61

2.2 Optimization of the Golfer-Club Model 63

2.2.1 Customizing the Golfer to Fit the Club 64

2.2.2 Customizing the Club to Fit the Golfer 66

2.3 Simulated Thought Experiments Using the Golfer-Club Model 67

2.3.1 Use of a Perfectly Rigid Shaft 67

2.3.2 Repositioning the Clubhead Center of Mass 68

2.3.3 Removal and Isolation of Radial Force 69

2.3.4 Effect of Shaft Deflection on Clubhead Orientation 71

$\begin{array}{lll}2.4 & \text { Model Validation } & 72\end{array}$

$\begin{array}{lll}2.4 .1 & \text { External Validity } & 72\end{array}$

2.4.1.1 Live Golfer Testing $\quad 72$ 
3.1 Optimization of the Golfer-Club Model

3.1.1 Typical Swing Results

3.1.2 Customizing the Golfer to Fit the Club

3.1.3 Customizing the Club to Fit the Golfer

3.2 Simulated Thought Experiments Using the Golfer-Club Model

3.2.1 Use of a Perfectly Rigid Shaft

3.2.2 Repositioning the Clubhead Center of Mass

3.2.3 Removal and Isolation of Radial Force

3.2.4 Effect of Shaft Deflection on Clubhead Orientation

3.3 Model Validation

3.3.1 External Validity

3.3.1.1 Live Golfer Testing

3.3.1.2 Error and Reliability of Live Golfer Testing

4.1 The Relationship Between Shaft Stiffness and Clubhead Speed

4.2 The Relationship Between Shaft Stiffness and Clubhead Orientation 
4.4 The Role of Radial Force

4.5 Limitations of the Model

130

4.6 Limitations of the Live Golfer Testing

4.7 Conclusions

4.8 Future Directions

REFERENCES

APPENDIX A Consent Form, Ethical Approval

APPENDIX B Inertial Properties of Simulated Club Segments

APPENDIX C $\quad$ Autolev $^{\circledR}$ Code: 3DGOLFG.AL 


\section{LIST OF TABLES}

Table 1.4.1.1 Parameter values for two segment golfer model.

Table 2.1.1.1 Six-segment golfer-club model segment parameters.

Table 2.1.3.1 Stiffness coefficients for rotational springs of simulated drivers.

Table 3.1.2.1 Measures of shaft bending during the swing and at impact for 9 optimized golfer-club models.

Table 3.2.1.1 Clubhead speeds attained with rigid shafts and the highest clubhead speeds attained with any of the three flexible shafts for each of the three golfer models.

Table 3.3.1.1.1 Lead deflections and clubhead speeds at impact as determined from Perspective 1. Values are the average and standard deviations from three swings. Lead is presented in centimetres, and clubhead speed is given in meters per second.

Table 3.3.1.1.2 Toe-down deflections at impact as determined from

Perspective 2. Values are given in centimetres, and are the average and standard deviations for three swings. 


\section{LIST OF FIGURES}

Figure 1.2.1.1 Shaft deflection in the lead and toe-down directions.

Figure 1.2.5.1.1 Demonstration of the effect of tangential force on shaft bending.

Figure 1.2.5.1.2 Demonstration of the effect of radial force on shaft bending.

Figure 1.4.3.1

Schematic of two-segment golfer model.

Figure 1.4.3.2

Free-body diagram of two-segment golfer model.

Figure 1.4.5.1

Search space for a single variable optimization problem.

Figure 2.1.1.1

Six segment 3D golfer-club model.

Figure 2.1.1.2

The torso segment and arm segment were constrained to rotate in specific planes.

Figure 2.1.2.1 Calculation of lead/lag shaft deflection along the $y$ axis of the shaft. Identical methods were used for calculating toe-up/ toe-down deflection along the $x$ axis of the shaft.

Figure 2.1.3.1

Experimental set-up for determining shaft stiffness.

Figure 2.1.5.1 Graphical depiction of the generalized coordinates used in Autolev $^{\circledR}$ for developing the 3D golfer model. Diagram A is from a perspective looking along the negative TILT3> axis. Diagram B is from a perspective looking along the positive TILT1> axis. 
Figure 2.4.1.1.1 Set-up of live golfer testing from Perspective 1.

Figure 2.4.1.1.2 Set-up of live golfer testing from Perspective 2.

Figure 3.1.1.1 Output from the four muscle torque generators during the optimized execution of Golfer-Medium with Club-Regular.

82

Figure 3.1.1.2 Generalized coordinates of the golfer model as a function

of time. Shoulder rotation is provided relative to the angular position of the trunk and not in absolute terms as described in section 2.1.5 and in Appendix C.

Figure 3.1.2.1 Dynamic loft at impact from the 9 optimized simulations in which the timing of each golfer model's swing was fit to each of the clubs.

Figure 3.1.2.2 Dynamic close at impact from the 9 optimized simulations in which the timing of each golfer model's swing was fit to each of the clubs.

Figure 3.1.2.3 Toe-up/toe-down and lead/lag deflections during the optimized execution of Golfer-Medium with Club-Regular. The shaded area represents the time during which the clubhead was deflected in the lag direction.

Figure 3.1.2.4 Kick velocity during the optimized execution of GolferMedium with Club-Regular.

Figure 3.1.3.1 Resulting clubhead speed and clubhead speed minus penalties over a complete range of shaft stiffness for a single swing pattern from Golfer-Medium. 
Figure 3.1.3.2

Figure 3.1.3.3

Figure 3.2.1.1

Figure 3.2.2.1

Figure 3.2.2.2
Resulting clubhead speed and clubhead speed minus penalties over a complete range of shaft stiffness for a single swing pattern from Golfer-Slow.

Resulting clubhead speed and clubhead speed minus penalties over a complete range of shaft stiffness for a single swing pattern from Golfer-Fast.

Angular velocity of the most proximal club segment for the optimized simulations of Golfer-Medium with Club-Stiff and Golfer-Medium with Club-Rigid.

Toe-up/toe-down deflections for three different clubhead center of mass positions. Golfer-Medium/Club-Regular was used for all three conditions. Normal refers to a standard driver that has the center of mass located in the toe-up direction relative to the shaft insertion point. In-line refers to having the center of mass collinear with the shaft. Reversed refers to having the center of mass in the toe-down direction relative to the shaft.

Lead/lag deflections for three different clubhead center of mass positions. Golfer-Medium/Club-Regular was used for all three conditions. Normal refers to a standard driver that has the center of mass located in the lag direction relative to the shaft insertion point. In-line refers to having the center of mass collinear with the shaft. Reversed refers to having 
the center of mass in the lead direction relative to the shaft. $\quad 100$

Figure 3.2.3.1 Comparison of shaft deflections between the simulated swing of Golfer-Medium with Club-Regular and the same swing with

radial force removed.

Figure 3.2.3.2 Shaft deflection when radial force acted in isolation of all other forces and torques supplied by Golfer-Medium to Club-Regular during the optimized swing.

Figure 3.2.3.3 Radial force acting along the $z$ axis of the most proximal club segment during the optimized swing of Golfer-Medium with Club-Regular.

Figure 3.2.4.1 Three-dimensional plot of dynamic loft as a function of both lead and toe-down deflections.

Figure 3.2.4.2 Three-dimensional plot of dynamic close as a function of both lead and toe-down deflections.

Figure 3.3.1.1.1 In-plane shaft deflection during the downswing of Live Golfer 4 with Club-Regular as captured from Perspective 1. 113

Figure 3.3.1.3.1 Angle of the swing plane relative to the horizontal for the optimized swing of Golfer-Medium with Club-Regular.

Figure 4.7.1
A. Position at start of downswing. B. Torso rotation.
C. Shoulder abduction. D. Wrist adduction. E. Wrist
adduction from behind. F. Rotation of the lead arm
about its longitudinal axis. Note: the above sequence 
is not from an optimized swing, but rather was generated solely to demonstrate specific movements.

Figure 4.7.2

A. At the start of the downswing, gravity tends to pull the club below the plane formed by arm abduction. B. Midway through the downswing, a component of force acting within the arm abduction plane produces an angular impulse on the club about the longitudinal axis of the lead arm.

Figure 4.7.3

If the club is above the arm abduction plane midway through the downswing, a component of force acting within the arm abduction plane produces an angular impulse on the club about the longitudinal axis of the lead arm which tends to rotate the club back down into the plane. 
1

INTRODUCTION TO THE STUDY 


\subsection{Introduction}

Supplying golfers with equipment is a lucrative business. Since the beginning of golf, the golf club has been modified to improve performance. Currently, large golf club manufacturers are leading the search for new club technologies. This is a very competitive market and companies are required to continuously provide improved designs to attract the consumer. In response to the evolution of design changes, governing bodies such as the United States Golf Association have imposed regulations limiting the performance enhancing modifications to golf clubs (USGA, 2004). Golf club manufacturers will no longer be able to claim that their club will hit the ball further than the competition. Without their main selling point, manufacturers will have to use new strategies to attract consumers.

One strategy focuses on customizing the stiffness of a golf club's shaft to an individual's swing in order to attain maximum shot distance. Maximum shot distance is achieved by generating the highest possible ball speed while imparting the optimal trajectory and spin rate for the particular ball speed attained. Shaft stiffness influences all three of these parameters. The predominant factor in generating maximum ball speed is attaining maximum clubhead speed at impact (Van Gheluwe, Deporte, \& Ballegeer, 1990). Theoretically, clubhead speed can be increased without changing the golfer's swing if the behaviour of the golf shaft is optimized. During the downswing, the shaft could bend backwards storing strain energy. This strain energy could then be converted to kinetic energy at impact. This kinetic energy would result in additional clubhead speed. The trajectory and spin rate of the golf ball after impact are strongly influenced by the orientation of the clubhead at impact. The orientation of the clubhead can be 
changed by altering the stiffness of the shaft. Therefore, theoretically, the optimal trajectory and spin rate can be generated by finding the shaft stiffness that produces the optimal clubhead orientation at impact.

A complete understanding of the shaft's role in executing a golf shot has been hindered by two factors. The first is a continuous barrage of performance claims by manufacturers that seem to be based more in marketing hype than scientific research. The second is the logistical difficulties that arise when attempting to quantify the influence of the shaft on the resulting flight of the golf ball. The main focus of this thesis is to gain an understanding of the role that shaft stiffness plays during the golf swing. This will predominantly be attempted through the use of mathematical modeling and computer simulation techniques in an effort to circumvent the difficulties of controlling extraneous factors when using live experimental testing. The influences of other shaft characteristics seem to be well defined. For example, lighter shafts can be swung with higher swing speeds than heavier shafts of the same length. Also, for a given mass and moment of inertia, a longer shaft will result in higher clubhead speed. The function of shaft bending in the golf swing is the least understood.

The stiffness of a shaft can, in theory, exert its influence on the resulting ball flight in two ways. The first involves the shaft's ability to store and subsequently release energy. During the down swing the shaft has the ability to bend and store strain energy which could be returned later in the swing in the form of kinetic energy, and potentially result in an increase in clubhead speed at impact with the ball. This increase in clubhead speed would increase ball speed and, therefore, shot distance. The second way the shaft can influence the resulting flight of the ball is by altering the orientation of 
the clubhead relative to the ball at impact. The orientation of the clubhead will not only affect the direction of ball flight, but will also affect the distance the ball travels by changing the launch angle relative to the horizontal, and the spin rate of the ball.

\subsection{Literature Review of Shaft Bending}

\subsubsection{Shaft Bending During the Swing}

Prior to impact with the ball, the shaft can be measured bending about three orthogonal axes fixed at the grip end of the club (Fig. 1.2.1.1). The $y$ axis is oriented from the back to the face of the clubhead, and the $x$ axis from the heel to toe of the clubhead. Twisting about the longitudinal axis ( $\mathrm{z}$ axis) of the shaft can also occur. Compared to the magnitude of deflection about the other axes, twisting about the longitudinal axis has a negligible influence on both the orientation of the clubhead (Butler \& Winfield, 1994) and its velocity at impact and therefore will not be considered

in this thesis. Further, only deflection occurring along the $y$ axis from the back to the face of the clubhead can contribute to clubhead speed. 


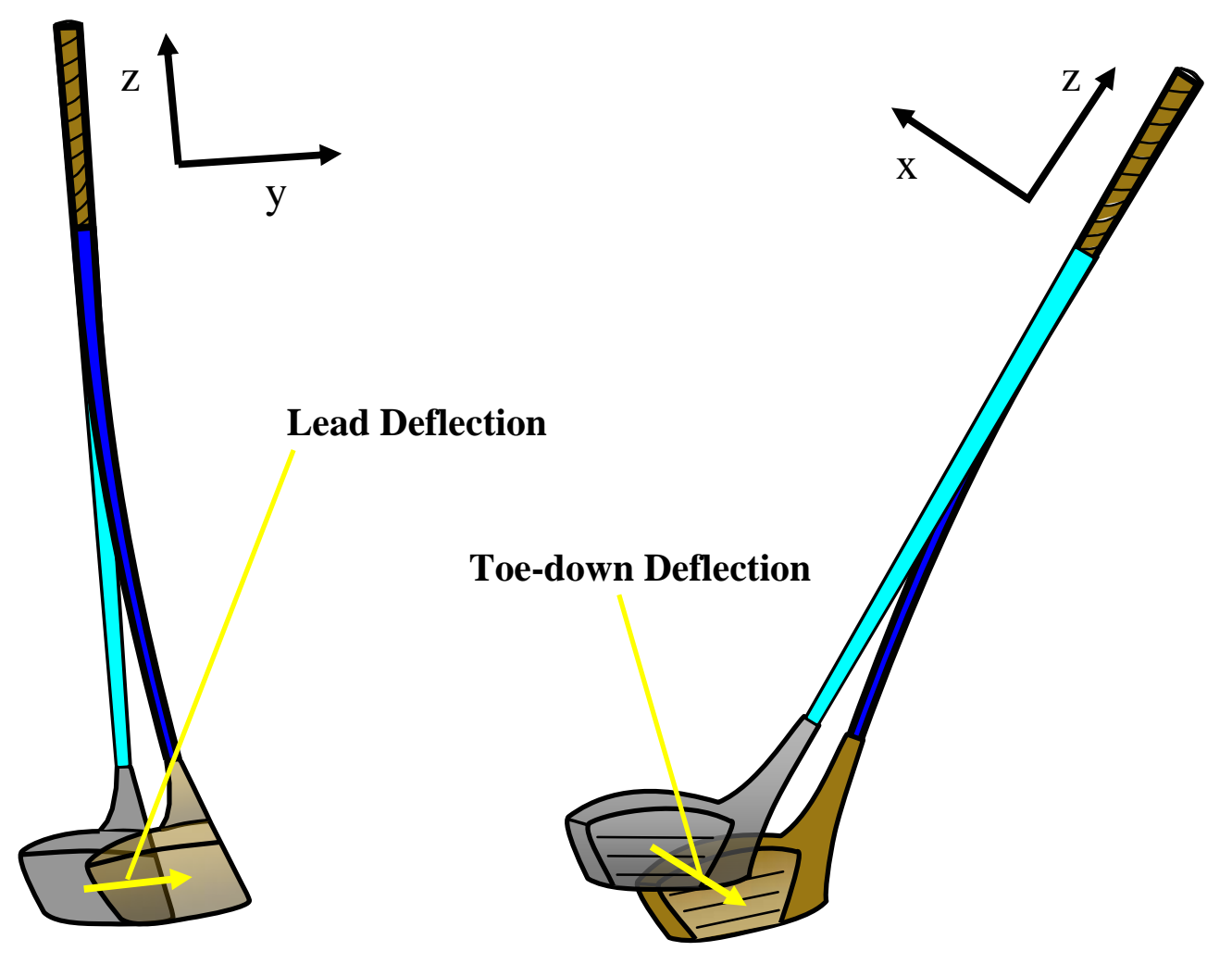

Figure 1.2.1.1 Shaft deflection in the lead and toe-down directions.

For the following descriptions, the club should be pictured in its address position as shown in Figure 1.2.1.1. The term lag will be used to describe the clubhead if it is deflected in the negative $y$ direction relative to the neutral shaft position. The term lead will be used to describe the clubhead if it is deflected in the positive $y$ direction relative to the neutral shaft position (Fig. 1.2.1.1). Although shaft deflection along the heel/toe axis cannot contribute to clubhead speed, it can have an influence on ball flight trajectory. The term toe-down will be used to describe the clubhead if it is deflected in the negative $x$ direction relative to the neutral shaft position. This is often referred to as droop in the golfing literature. The term toe-up will be used to describe the clubhead if it is deflected in the positive $x$ direction relative to the neutral shaft position. It should 
be emphasized that these conventions are defined relative to a three-dimensional coordinate axis fixed in the grip end of the club.

Butler and Winfield (1994) used strain gauges attached to the shaft near the grip end to measure bending during the swing. The strain gauges were calibrated so that a measure of shaft deflection could be predicted from the strain values in the shaft. This procedure was used to determine shaft bending during the swings of several golfers. They measured peak deflection values in the lag direction as large as $7 \mathrm{~cm}$, and peak deflections in the toe-up direction greater than $15 \mathrm{~cm}$. For three golfers swinging the same club, and each with a clubhead speed of $46 \mathrm{~m} / \mathrm{s}$, toe-down deflections at impact ranged from approximately $0.5-5 \mathrm{~cm}$, while lead deflections at impact ranged from approximately $0.3-4 \mathrm{~cm}$.

Mather and Cooper (1994) employing a photogrammetric procedure found that, for a good player swinging a driver, both the lead and toe-down deflections at impact can be as large as $5 \mathrm{~cm}$. They also reported that during the swing of a poor golfer the shaft tends to bend a greater amount in both directions when compared to a good golfer due to greater torque amplitudes applied to the club by the poor golfer. This implies that the shaft bending occurring during the poor player's swing would be greater than $5 \mathrm{~cm}$ due to the larger torque amplitudes. However, Mather and Cooper did not state in which direction relative to the shaft that the greater amounts of bending would occur, or during which point in the downswing.

Horwood (1994) measured bending and stress amplitudes "in the plane of the swing” with the use of strain gauges. He found that during the backswing the shaft was bent forward due to a bending moment of $9 \mathrm{Nm}$ and on the downswing, the shaft was 
bent backwards due to a bending moment of $6 \mathrm{Nm}$. Horwood stated that these values are typical regardless of shaft design or golfer ability. For one golfer swinging an $\mathrm{S}$ (i.e., stiff) flex shaft with a clubhead speed of $42.5 \mathrm{~m} / \mathrm{s}$ at impact, the clubhead moved through $12.7 \mathrm{~cm}$ from its maximum lagging position into its maximum leading position. Horwood also stated that the lead deflection present at impact is primarily a function of centripetal force acting on the offset position of the center of gravity of the head and inertial forces. Horwood did not go into detail to describe the inertial forces.

There are a few criticisms that can be made regarding Horwood's (1994) findings. First, Horwood found that the bending moments in the shaft were greater during the backswing. It seems doubtful that the bending moments would be greater in the backswing. Just a qualitative analysis of the golf swing would bring this statement into question. Second, in opposition to Horwood's claim that the torque values are typical regardless of golfer ability; other researchers have found that different golfers can possess different swing characteristics which would alter the bending moments in the shaft (Mather \& Cooper, 1994; Cooper \& Mather, 1994). This will be discussed in greater detail in a later section. Finally, due to the $90^{\circ}$ rotation of the shaft about the longitudinal axis of the lead arm during the downswing, it is not clear what Horwood meant by "in the plane of the swing". The bending moments reported may be in the toeup/toe-down direction, or in the lead/lag direction. However, it is likely that Horwood combined the bending in both directions in some way to determine the bending occurring in the swing plane.

Perhaps the most cited study regarding the role of the shaft in the golf swing is that of Milne and Davis (1992). Milne and Davis examined the role of the shaft using 
both computer simulation and experimental strain gauge measurements. The presentation of their shaft bending results was ambiguous. One set of sketches, depicting shaft deformation during the computer simulation, showed maximum deflections of approximately $2.5 \mathrm{~cm}$, yet a graph of the computer simulation values clearly showed in-plane deflection values exceeding $10 \mathrm{~cm}$. It is possible that the discrepancy was due to different measurement conventions for the same phenomenon. No explanation was given regarding how values for the sketches were measured, but the paper implies that the deflection values presented in the graph were measured relative to a tangent to the butt end of the club, thus representing clubhead lead/lag deflection. This latter technique follows the same convention stated earlier in this thesis regarding lead/lag and toe-up/toe-down deflections. Milne and Davis presented no deflection values for the experimental strain gauge data, however, they did present shaft bending moment data from the live tests which closely agreed with the bending moment data generated by their simulations. Therefore, it could be interpreted that their live golfer shaft deflections also reached magnitudes of $10 \mathrm{~cm}$.

Based on the above findings it can be stated that the shaft does bend during the swing. The exact amount of deformation seems to depend primarily on the swing pattern generated by the golfer, and in part on the flexibility of the shaft. The bending occurring in the downswing suggests the shaft can store and release energy, potentially increasing clubhead speed at impact. 


\subsubsection{Utilization of Energy Stored in the Shaft}

When a flexible material, such as a golf shaft composed of graphite or steel is deformed, potential energy is stored in the material in the form of strain energy. This energy can be translated into kinetic energy. If this phenomenon does occur during the down swing, the following sequence would be expected. Early in the downswing, the golfer applies forces and torques at the grip end of the club that accelerate the club in the downswing direction. The mass of the clubhead at the distal end of the shaft would provide an inertial resistance to the motion of the shaft during the downswing. This inertial resistance would result in shaft bending and the storage of strain energy in the structure of the shaft. At some point later in the swing, the stored strain energy would be transferred into kinetic energy and the clubhead would move from lagging behind the grip end into a leading position. If this happened quickly, just prior to impact, an increase in clubhead speed over that of a rigid shaft would be expected. This addition to clubhead speed in the lead direction is referred to as kick velocity.

Milne and Davis (1992) concluded that shaft flexibility does not play an important dynamic role in the golf swing. It is unclear how this decisive conclusion was reached. As stated previously, their results suggest the clubhead is deflected considerably during the downswing, which implies the storage of strain energy and the possibility of kick velocity adding to the overall clubhead speed. However, there is no mention of kick velocity in the paper or how the aforementioned bending affects clubhead speed at all. Certainly, there should be some form of operational definition that can be used as the measuring stick to determine the importance of shaft flexibility. Milne and Davis provide no such device. The obvious variable would be kick velocity. 
It could be stated a priori, that kick velocity needs to exceed a nominal percentage of total clubhead speed in order for the shaft to be considered as playing an important role in producing higher ball speed. If this percentage were not reached, then the shaft's role in producing higher clubhead speed would be deemed negligible.

There is an important validity concern with the mathematical model developed by Milne and Davis (1992) that stems from their attempt to model the 3D nature of shaft dynamics. Milne and Davis realized that an essential requirement of a simulation of shaft bending was that it be 3D. They stated that the main reason for this is that the center of mass of the clubhead does not lie on the shaft. Therefore, they "allow" for the rotation of the clubhead about the longitudinal axis of the lead arm in the plane of the swing. Although presented in a vague fashion, it appears that clubhead rotation about the longitudinal axis of the lead arm was incorporated into the simulation in the following way. From live golfer tests, the distance of the center of mass of the clubhead from the shaft in the swing plane was determined as a function of the angular position of the shaft relative to the vertical. The center of mass of the clubhead was then constrained in their 2D simulations to change its position relative to the shaft as a function of wrist angle. Although Milne and Davis should be commended for being the first to make an attempt at representing the 3D nature of the golf swing, I will describe how their incomplete attempt at 3D modeling inevitably led to their unfounded conclusion.

Milne and Davis (1992) developed a pseudo-forward dynamics model which resulted in an incomplete simulation of shaft dynamics during the swing. A true forward dynamics model would have forces and torques acting as inputs on a system 
whose inertial properties are sufficiently defined for the number of dimensions that the system was intended to represent. The kinematics of every point, particle or body will be completely determined by the input kinetics according to the laws of classical dynamics. Milne and Davis developed a 2D forward dynamics model in an attempt to resolve a 3D dynamics problem. The applied torques in the system acted in a single plane, and the inertial properties of the system's segments were only expressed for motion in a single plane. According to classical dynamics, the change in motion of a body does not occur without the application of a force. In reality, some mechanism must cause the clubhead to rotate about the longitudinal axis of the lead arm in the plane of the swing. This mechanism would also have an effect on shaft bending. This mechanism was not represented in the model employed by Milne and Davis and therefore its effect on shaft bending cannot be evaluated. A hypothetical thought experiment will further clarify my point.

Consider the position of a golfer-club system at three-quarters of the way into the downswing, just as the shaft begins to rotate about the longitudinal axis of the lead arm through $90^{\circ}$ to square up the clubface for impact. At this point, the center of mass of the clubhead will be at some distance away from the longitudinal axis of the lead arm. Assume now, that the golfer exerts a torque on the club about the longitudinal axis of the lead arm in an attempt to square the clubhead for impact. This torque will generate a bending moment in the shaft and likewise create some magnitude of shaft deflection not evident in the plane of the swing, but still in the lead/lag direction relative to the clubface. The dynamic effects of this action on shaft bending cannot be ignored and will certainly influence shaft deflection in the lead/lag direction as the clubhead moves 
into a square position at impact. It is precisely a mechanism such as this that cannot be represented by the model of Milne and Davis.

Therefore Milne and Davis (1992) concluded that shaft bending was solely a result of centripetal forces acting on an eccentrically placed center of mass. This is not surprising since their model was not capable of arriving at any other conclusion. In summary, the study conducted by Milne and Davis lacks the methodological structure to support their hypothesis and therefore, a sound objective conclusion could not have been reached with their model.

Miao, Watari, Kawaguchi, and Ikeda (1998) investigated clubhead speed as a function of grip speed for a variety of shaft flexibility. Three sets of tests were conducted; computer simulation, robotic, and live golfer. Separate graphs of clubhead speed versus grip speed were plotted for each shaft flex. For both, the simulation and machine results, clubhead speed oscillated in a systematic fashion above and below a line representing the linear relationship between grip speed and clubhead speed assuming a rigid shaft. For a given grip speed, clubhead speed at impact varied depending on the shaft stiffness. In the live golfer tests, Miao et al. reported that clubhead speeds reached a peak at sub maximum grip speed values. On the surface, the results of Miao et al. seem definitive, however, several criticisms of both their methods and their interpretation of results can be argued.

The simulation tests conducted by Miao et al. (1998) suffer from the same limitations as Milne and Davis (1992) in addition to some other concerns. Their mathematical model is completely 2D in nature and makes no attempt at incorporating the $90^{\circ}$ rotation of the club, about the lead arm, during the downswing. Therefore, the 
effects of lead/lag deflections occurring prior to and during the rotation of the clubhead into a square position cannot be represented. Their model has also incorporated two further assumptions that stem from an attempt to match their simulated data to their robotic tests. First the acceleration of the grip end of the club was fixed at a constant value based on the robotic tests. It is doubtful that many golfers accelerate the club at a constant value during the downswing. Mather and Cooper (1994) showed that the angular acceleration of the club in the plane of the swing ranged from 0 to approximately $400 \mathrm{rad} / \mathrm{s}^{2}$ during the downswing. Their second assumption was that the shaft of the club was considered to have no damping properties. While this assumption is likely reasonable for a club rigidly fixed at the grip end (such as a robotic test), it is not so reasonable considering the grip of a live golfer. The hands of the golfer would certainly introduce some damping into the system (Jorgensen, 1994; Mather \& Cooper, 1994; Okubo \& Simada, 1990). This concern about the damping effects of a human grip also applies to their machine tests. It was also not clear if their swing machine incorporated the rotation of the club about the longitudinal axis of the lead arm during the downswing. A final point regarding their interpretation of results is directed at their live golfer tests. Miao et al. (1998) reported that clubhead speeds reached peak values at sub maximal grip speeds. However, there was no mention of the magnitude of error associated with their measurement methods. This is especially questionable after inspection of their scatter plots of grip speed versus clubhead speed. It is evident that the "peak" clubhead speeds differed from the clubhead speeds attained at the highest grip speeds by no more than $0.2 \mathrm{~m} / \mathrm{s}$. Yet, from these same plots, it is clear that clubhead speed varied by as much as $3 \mathrm{~m} / \mathrm{s}$ for a given grip speed with the same club. 
Since a difference of $0.2 \mathrm{~m} / \mathrm{s}$ seems to fall within the range of error, the live golfer results presented by Miao et al. are inconclusive. However, other researchers have attempted to quantify the actual contribution of kick velocity to clubhead speed at impact.

Using experimental strain gauge data, Butler and Winfield (1994) calculated kick velocities at impact that ranged from $2.27 \mathrm{~m} / \mathrm{s}$ to $2.48 \mathrm{~m} / \mathrm{s}$. This was approximately $5 \%$ of the total clubhead speed. There appeared to be no obvious relationship between lead/lag deflection at impact and the corresponding kick velocity reported by Butler and Winfield. Horwood (1994) had similar findings using strain gauge data. He determined that the maximum kick velocity was $5 \%(2.01 \mathrm{~m} / \mathrm{s})$ of the total clubhead speed of 42.5 m/s. However, Horwood theorized from his data that kick velocity would not significantly contribute to clubhead speed. He reasoned that since the clubhead had close to its largest leading deflection at impact, the kick velocity at this point would be

close to zero. It should be noted that neither of these studies made any attempt to customize the flex of the golf shafts to the test subjects' swings. It seems plausible that if the shaft behaviour was optimized to the individual swings, the kick velocities found might be higher than $5 \%$ and could be timed to occur at impact with the ball.

\subsubsection{Variations in Force and Torque Patterns applied to the Club}

If all golfers had swings that produced the same kinetic patterns to the golf club, then only one shaft stiffness would be required for maximized kick velocity. A shaft's behaviour is a reflection of the kinetic profile applied throughout the swing. If golfers swing the club differently, then this results in varying kinetic applications. These varied 
force and torque applications would result in varied shaft behaviours. However, these varied shaft behaviours could theoretically be assimilated into the optimized condition, of attaining maximum kick velocity at impact, by altering shaft stiffness to match the torque profile of the golfer. Several studies provide evidence that kinetic patterns are specific to the golfer, and therefore, shaft stiffness can potentially be altered to optimize kick velocity.

Butler and Winfield (1994) measured the shaft bending profiles of three golfers using the same club. The profiles were different, yet all swings generated a clubhead speed of $46 \mathrm{~m} / \mathrm{s}$. Three variables measured were load up time, peak deflection, and time at peak deflection. Load-up time is defined as the time from impact to the start of loading of the shaft. Peak deflection is the maximum deflection of the shaft in the toe up/down direction and the time at peak deflection is the amount of time for the shaft to reach its peak deflection during the downswing. For the three golfers, load up time ranged from $0.39 \mathrm{~s}$ to $0.63 \mathrm{~s}$, peak deflection ranged from $9.2 \mathrm{~cm}$ to $15.7 \mathrm{~cm}$, and time at peak deflection ranged from $0.23 \mathrm{~s}$ to $0.51 \mathrm{~s}$. These values indicate that the timing and amplitude of the kinetics applied by the three golfers are very different. In response to these different torque patterns, the golf club behaved differently for each golfer.

Other researchers support the contention that golfers swing differently and that in general, a poor golfer shows greater amplitudes and more fluctuations in torque than a good golfer (Cooper and Mather, 1994; Mather and Cooper, 1994). According to Mather et al. (2000) the professional golfer generates as much as $80 \%$ of their clubhead speed in the last stages of the swing. In contrast, the high handicap golfer reaches $120 \%$ of their final impact velocity early in the downswing, slowing appreciably before 
impact. If this is the case, then a golf shaft will behave differently depending on the skill of the golfer swinging the club. A good golfer might smoothly accelerate the club, while a poor golfer would produce acceleration initially, followed by deceleration prior to impact.

\subsubsection{Changes in Clubhead Orientation due to Shaft Deflection}

Although it is generally accepted that the orientation of the clubhead relative to the ball is altered due to shaft bending near impact, very few studies have attempted to quantify the effects. Mather and Cooper (1994) stated that depending on the geometry of the shaft, a lead deflection of $5 \mathrm{~cm}$ can result in a $5^{\circ}$ increase in the loft of the club. They refer to this added loft as dynamic loft. Horwood (1994) explained that increasing the lead deflection at impact would increase the dynamic loft at impact and result in a higher ball trajectory. Although not explicitly reported by any researchers, bending in the toe-up/toe-down direction will also result in altered ball flight. However, these effects have not been reported.

\subsubsection{Cause of Shaft Bending from a Newtonian Perspective}

The resultant force and torque vectors applied by the golfer at the grip end of the club are continually changing in both magnitude and direction in 3D space during the downswing. It is solely these two factors, along with gravity, that cause the shaft to bend during the downswing. To simplify the situation, given a point of rotation, torques can be replaced by appropriately placed forces without any changes in the dynamics of the system. Therefore, it can be stated that the forces applied at the grip end of the club 
are responsible for shaft bending during the downswing. It is my opinion that a clearer understanding of the source of shaft bending can be gained by resolving the resultant force, applied at the grip end of the club, into a tangential component and a radial component. The tangential component would act in a plane formed by the $x$ and $y$ axes, while the radial component would act along the $z$ axis (Fig. 1.2.1.1).

\subsubsection{Tangential Force Component}

The tangential force component acts perpendicularly to the shaft at the grip end of the club. This component serves to linearly accelerate the golf club into impact with the ball. Consider a simplified model of a golf club. Two uniform rigid links connected by a revolute joint that is spanned by a rotational spring-damper element (Fig. 1.2.5.1.1). A tangential force is applied at the top of link 1 to accelerate the club, while a constraint torque is also applied at the top of link 1 to prevent its rotation. As a result, the top of link 2 will experience a positive tangential force. This force at the top of link 2 will result in torque acting about the center of mass of link 2 which will tend to rotate the link clockwise. The link rotates because the mass of the clubhead at the distal end of the link provides an inertial resistance to the linear motion of the shaft. The rotation of link 2 will stretch the rotational spring-damper until an equilibrium position is reached with link 2 fixed in some "lagging” position behind link 1. If at some point the tangential force at the top of link 1 is removed, the torque from the spring will briefly rotate link 2 into a "leading" position ahead of link 1 before eventually returning link 2 into a straight line with link 1. From a work-energy perspective, the stretching of the spring would result in the storage of strain energy that would be converted into kinetic energy once 
the tangential force at the top of link 1 was removed. The findings of Miao et al. (1998) and Jorgensen (1994) were primarily based on the effects of the tangential force applied at the grip. This was a result of the mathematical models they employed in generating their findings. Their mathematical models did not incorporate an off-set clubhead center of mass and therefore, the effects of a radial force component could not have been fully realized.

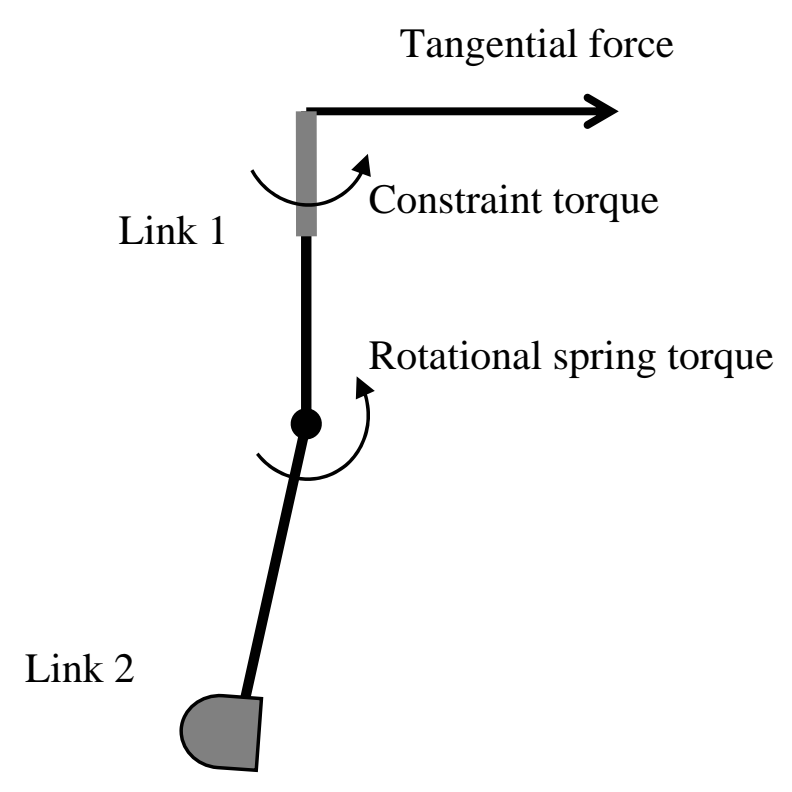

Figure 1.2.5.1.1 Demonstration of the effect of tangential force on shaft bending

\subsubsection{Radial Force Component}

Force is not just applied at a tangent to the shaft. Radial force is also applied along the longitudinal axis of the shaft to maintain the club's circular path during the downswing. This radial force applied by the hands at the grip end of the club can also cause the shaft to bend (store strain energy). This bending is due to the offset of the 
center of gravity of the clubhead relative to the line of action of the radial force along the longitudinal axis of the shaft. Consider a similar scenario to the one explained in section 1.2.5.1. Two uniform rigid links are connected by a revolute joint that is spanned by a rotational spring-damper element (Fig. 1.2.5.2.1). A vertical (representing radial) force is applied at the top of link 1 to accelerate the club, while a constraint torque is also applied at the top of link 1 to prevent its rotation. As a result, the top of link 2 will experience a positive vertical force. This force at the top of link 2 will result in torque acting about the center of mass of link 2, due to its offset, which will tend to rotate the link counter-clockwise. This rotation of link 2 will stretch the rotational spring-damper until the torque from the spring equals the torque due to the radial force. Note that it is not possible for the radial force to pull the center of mass of link 2 into a stable position passed the line of action of the radial force. The nature of this bending will tend to pull the clubhead into a leading and toe-down position as impact approaches. An increase in clubhead speed at impact due to the release of this stored energy is not possible. The strain energy, stored as a result of the radial force, could only be released prior to impact if the golfer were to let go of the club. Even if the stored energy could be released, it would result in a negative kick velocity. It should be noted that the comments in this section describe how the shaft would behave if the radial force acted in isolation of any tangential force applied to the grip end of the club. 


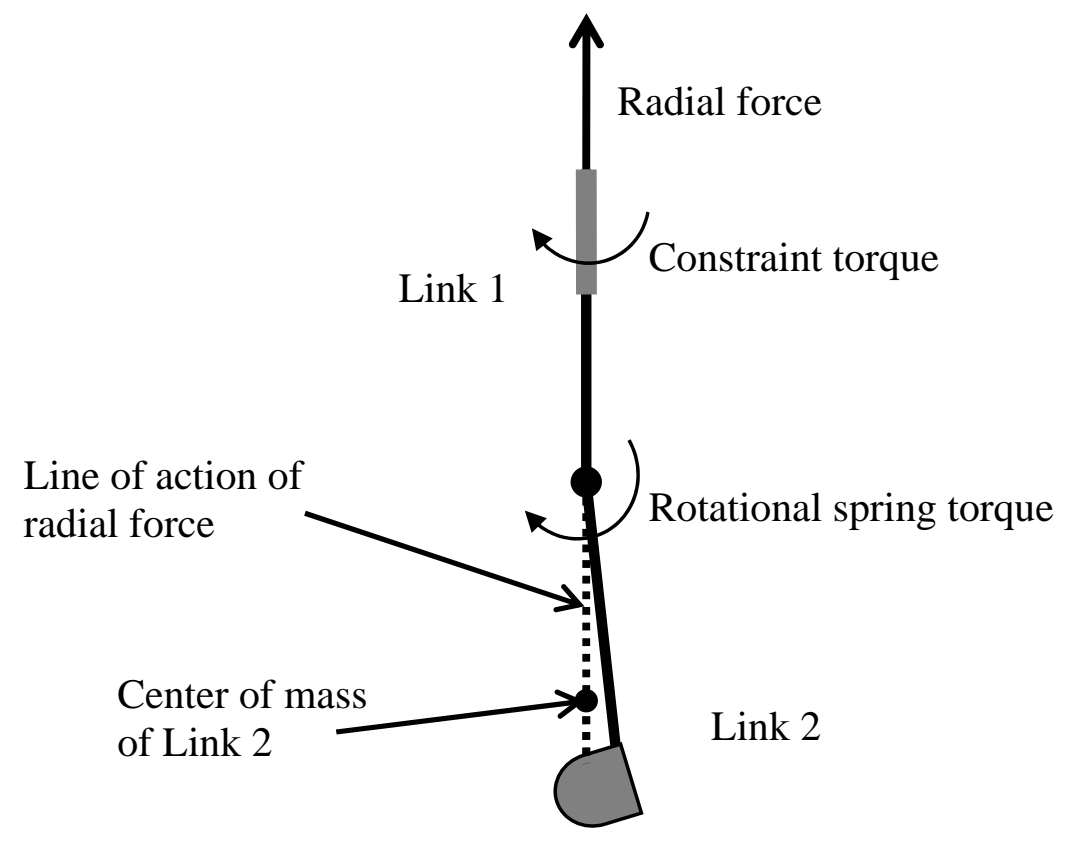

Figure 1.2.5.1.2 Demonstration of the effect of radial force on shaft bending.

\subsubsection{Optimization of Shaft Behaviour}

Optimization is a process that results in a best case outcome of a dependent variable through the manipulation of one or more independent variables. For the purpose of the golf drive, maximum horizontal clubhead speed at impact is desired (Van Gheluwe, Deporte, \& Ballegeer, 1990). Other factors such as impact quality, ball launch angle, and ball spin rate also play a role. Theoretically, the shaft can contribute to clubhead speed by providing a maximum amount of kick velocity at impact. Thus, shaft behaviour is optimized when the dependent variable, kick velocity, is maximized at impact.

According to Butler and Winfield (1994), kick velocity is greatest when the shaft is straight at impact because the kinetic energy is maximized. This statement is in agreement with the characteristics of an oscillating spring system and is supported by 
other researchers (Horwood, 1994). Jorgensen (1994) in his book 'The Physics of Golf' states that the shaft does not have to be straight in order for kick velocity to be maximized. He determined this through the use of a mathematical model. His conclusion lacks an adequate description of methodology and a presentation of the results supporting the claim. Jorgensen also fails to provide a theory for how this physical phenomenon could occur. One possible explanation of Jorgensen's finding could be related to the contribution of shaft bending due to the off-set position of the clubhead center of mass relative to the shaft. In combination with some 'spring-back' effect, this may lead to a kick velocity that increases in magnitude past the mid point of the shaft's oscillation. Unfortunately, Jorgensen's did not incorporate an offset clubhead center of mass into his model. Jorgensen explained that bending due to the offset center of mass was a function of centripetal acceleration. He then curiously states that it will only have an influence early in the swing, while later its effect will be negligible. He therefore neglects incorporating it into his model.

A second factor that should be considered when optimizing the behaviour of a shaft is its influence on the orientation of the clubhead at impact with the ball. Based on the aerodynamics of the golf ball, there will be an optimal launch angle and spin rate that the ball should possess for it to travel the maximum horizontal distance. The angle of the clubface, relative to a horizontal line extending to the target, at impact will affect both the launch angle and spin rate of the golf ball after impact. 


\subsection{Statement of Problem and Hypothesis}

\subsubsection{The Problem}

How does the stiffness of a golf club shaft affect clubhead speed and clubhead orientation at impact with the ball?

\subsubsection{Research Hypotheses}

1. A golfer-club system can be simulated with a 3D, six-segment mathematical model driven by muscle torque generators.

2. Golf shafts bend during the golf swing as a result of both tangential and radial forces relative to the grip end of the club during the downswing.

3. Customizing the flexibility of a golf shaft to a specific swing will increase clubhead speed at impact, but not by a meaningful amount $(<1 \mathrm{~m} / \mathrm{s})$.

4. Clubhead orientation at impact will depend upon both swing speed and shaft stiffness.

5. Altering the position of the center of mass of the clubhead relative to the shaft will affect the dynamics of the golf club during the downswing.

From the review of literature, computer modeling appears to be the best research tool available to address these hypotheses. The following section will provide a conceptual understanding and literature review of the modeling methods used in this thesis. 


\subsection{Review of Optimized Forward Dynamic Simulations}

Forward dynamic simulations provide an alternative to researchers attempting to answer questions about human movement. Forward dynamic simulations offer some advantages over more traditional methods of human movement inquiry.

One goal for biomechanists is to gain an understanding of how the coordinated contraction of the body's muscles can lead to the best execution of a skill. In achieving this goal the kinematics of the movement must be measured and attempts made at quantifying the underlying kinetics. A major stumbling block is the error associated with the techniques biomechanists use in their measurements. Often the associated error can obscure true measurement values to a point that renders findings inconclusive.

To answer specific questions, the biomechanist might want to measure the effect of changing one variable while holding all other variables constant. For example, if a golf swing is perfectly executed every time, what effect will the flexibility of the club's shaft have on the resulting clubhead speed at impact? It is difficult for even an elite performer to execute a skill in an identical manner over many trials. In particular, if relatively small changes in the outcome are practically significant, then the performer must have even more precision of repeatability in executing the skill. Further, considering the golfing example, it is even more difficult for the researcher to know with certainty that the swing was executed in an identical manner.

A forward dynamics model overcomes some of the issues involved with live experimentation. Relative to live testing, there is essentially no error in measuring the outcome of a model, and due to a model's repeatability in executing a movement; it is 
very easy to isolate the effects of changing a single variable. However, forward dynamic models are particularly vulnerable to problems of external validity. In general, a more detailed model (incorporating more aspects of the real system) is more resilient to external validity threats. However, a more detailed model is more complex which inhibits the interpretation of the results. As such, there will always be a subjective estimate as to the best balance between parsimony, on one side of the scale, and goodness of fit on the other. It is easier to interpret the results of a simple model, but the 'correctness' of those results depend on the validity (internal and external) of the model. The level of complexity required by a model will depend upon the specific questions the researcher wants to answer. It is the nature of these questions that should dictate how the model is developed.

The development of an optimized forward dynamics model can be accomplished in five phases.

1. Develop the physical properties of each segment

2. Provide the model with the ability to generate motion

3. Develop equations that will govern the motion of the model

4. Determine how the equations will be solved

5. Implement an optimization scheme

The specific methods that were applied in developing the model used in this thesis may prove to be unnecessarily difficult for the reader to gain a conceptual understanding of an optimized forward dynamics model. Therefore, the development of a simpler 2D, 2-segment model will be illustrated. In each of the five phases, the 
simplest methods, conceptually, will be described, and other techniques previously used in research will be reviewed.

The development details of a model should be based on the specific question the researcher wants to answer. In this case, assume the researcher is interested in determining the optimal muscular control strategy in the execution of a golf swing. Specifically, what muscle coordination strategy at the shoulder and wrist should a golfer implement to achieve maximum clubhead velocity at impact with the ball? The purpose of this section is solely to provide a conceptual framework for optimized forward dynamic simulations.

\subsubsection{Develop the Physical Properties of each Segment}

First, the number of segments must be decided. Theoretically, the researcher should at least include all segments that show motion during execution of the real skill. However, this could result in motion equations that are too large for the current capabilities of standard desktop computers, and/or optimization search times that are not practical. Therefore, the researcher must make certain concessions based on educated assumptions. For example, the assumption could be made that the motion of the lower body and trunk has little influence on the muscle control strategy used at the shoulder and wrist. Although this may not be a realistic assumption, it allows the golfer to be represented by a model employing just the lead upper arm, forearm, and club. This supposition allows the model to be reduced to three segments. Perhaps, the researcher notices that during an actual golf swing, the forearm has no motion relative to the upper arm. This permits a second assumption, that the forearm and upper arm could be 
modeled together as a single segment. The model has now been reduced to two segments. The researcher must decide on the degrees of freedom that each segment of the model should possess.

The specific degrees of freedom chosen, will dictate whether the system can be modeled in 2D or 3D. The added complexity of a 3D model over a 2D model cannot be overstated. If a question can be answered with a 2D model, then a 3D model should not be employed just for the sake of comprehensiveness. Although it is likely that a golf swing does not occur perfectly in a single plane, it could be assumed that its deviation from a plane is not large enough to warrant the added complexity of a 3D simulation. Therefore, even though, an actual shoulder joint has three degrees of freedom, and a wrist joint has two degrees of freedom, their motion during a golf swing only requires a single degree of freedom from each. The assumption could be made that the motion of segment 1 (upper arm + forearm) and segment 2 (golf club) are both constrained to a single plane.

The decision on the model's degrees of freedom allows the inertial parameters of each segment to be estimated. Since only a 2D representation of the model is required, each segment can be represented by a single moment of inertia value that corresponds to an axis perpendicular to the plane of motion. If a segment moved in $3 \mathrm{D}$, then the moment of inertia must be represented about all three of the segment's principal axes. Lengths, masses, and distances to the center of mass for each segment must also be defined. There are several ways to calculate these physical characteristics for each segment. 
The easiest method for obtaining the necessary physical properties of segments is to simply refer to a reference that directly supplies these values in the form of means and standard deviations for a large group of individuals. Zatsiorsky (2002) supplies such data in tabular form. If the researcher wants to make some attempt at basing the segment's inertial properties on a particular individual, then regression equations based on the height and/or weight of an individual are also available (De Leva, 1996). The potential of more accurate inertial estimates of individuals is provided by the means of geometrical modeling (Hanavan, 1964; Hatze, 1980; Yeadon, 1990). Employing these methods, the body is broken down into segments that are represented as geometrical solids. Measurements are taken on actual individuals to determine the size and shape of each segment. Density values taken from the literature are then used to calculate moment of inertia values for each segment.

Suppose we are not interested in our model exactly representing a specific individual, and that we are satisfied with the knowledge that our model's physical properties easily fit within the norms of the human population. Therefore, an arbitrary golfer is chosen and three measurements are taken: height, mass, and arm length. Based on these measurements, regression equations are used to determine the moment of inertia and the position of the arm segment's center of mass relative to its proximal end (Table 1.4.1.1). Our model may not exactly represent the particular individual but its inertial values are certainly a reasonable approximation of the athlete's inertial characteristics for their arm. The physical properties for the golf club can be determined experimentally. Now that we have a physical representation of our model, we must provide our model with a means for motion. 
Table 1.4.1.1 Parameter values for two segment golfer model

\begin{tabular}{|c|c|c|c|c|}
\hline & $\begin{array}{c}\text { Mass (kg) } \\
\mathbf{M}_{\mathrm{i}}\end{array}$ & $\begin{array}{c}\text { Length (m) } \\
\mathbf{L}_{\mathrm{i}}\end{array}$ & $\begin{array}{c}\text { Center of Mass (m) } \\
\mathbf{r}_{\mathrm{i}}\end{array}$ & $\begin{array}{c}\text { Moment of } \\
\text { Inertia } \mathbf{( k g}^{*} \mathbf{m}^{\mathbf{2}} \mathbf{)} \\
\mathbf{I}_{\mathrm{i}}\end{array}$ \\
\hline $\begin{array}{c}\text { Segment 1 } \\
\text { Arm }\end{array}$ & 6.12 & .64 & .37 & .72 \\
\hline $\begin{array}{c}\text { Segment 2 } \\
\text { Club }\end{array}$ & 0.34 & 1.12 & .86 & .057 \\
\hline
\end{tabular}

$r_{i}$ is measured from the proximal end of the segment. $I_{i}$ is measured about the center of mass of the segment

\subsubsection{Provide the Model with the Ability to Generate Motion}

The researcher can exert varying levels of control over the means of motion generation. As the level of control increases, the depth at which the model provides insight into the real system decreases. For example, the researcher can specify an angular velocity function for each segment in the model. Essentially, this limits the researcher to only being able to predict geometric positions of the system and linear velocities as a function of simulation time. To take full advantage of a forward dynamics model, motion should be generated by forces and/or torques acting on the inertial properties of the segments.

Newton's second law explains that forces are the cause of changes in motion. The resultant force acting on an object produces an acceleration that is inversely proportional to the mass of the object. Over time, an acceleration results in a change in the object's velocity and an object's velocity determines its displacement. 
Conceptually, the same ideas hold true for angular kinetics and kinematics. The preceding statements in this paragraph are the foundation of forward dynamics. Aside from gravity, only the muscular activity at the shoulder and wrist will have the ability to provide energy for the golfer model through the generation of forces and/or torques. Forces and/or torques can be represented in a forward dynamics model with varying degrees of complexity.

At the simplest level, it is possible to represent the combined muscle actions at a joint with a single torque. Theoretically, this torque will produce the same motion as the combined effect of all individual muscles acting across the joint. A further simplification would be to assume that once activated, this joint torque instantaneously rises to its maximum value and remains at this value until it is deactivated. Similarly, constant muscle forces from individual muscles could be represented if the model was also provided with estimates for the muscle moment arm lengths. At this level of complexity, aside from estimating maximal force or torque values, no attempt is made at representing the behaviour of human muscle.

It has been previously determined that the tendon tension produced by a muscle depends on four factors: activation level, sarcomere length, velocity of muscle contraction, and previous contraction history. The level of muscle activation is time dependent. Essentially, it takes skeletal muscle a certain amount of time to reach its maximum potential force (Dudel, 1978). The amount of force production capable from a muscle also depends upon its length, or more specifically, the length of the sarcomeres that comprise the muscle. This can be explained using the sliding filament theory (Gordon, Huxley, \& Julian, 1966) which contends that maximal force occurs at a 
sarcomere's resting length when actin and myosin are optimally overlapped. In practice, it is difficult to determine at what joint configuration an individual muscle's sarcomeres are at resting length. Wilkie (1949) demonstrated that muscle force production was also a function of a muscle's rate of shortening. For concentric contractions, a muscle is capable of producing the most force at slow contraction velocities, while for eccentric contractions the greatest forces are produced at high contraction velocities. It has been demonstrated by Cavagna (1974) and Edman (1978) that a muscle will produce a greater concentric force of contraction if the muscle is immediately put on stretch prior to the concentric motion. This is referred to as the force enhancement phenomenon. Previously, researchers have attempted to incorporate these measured muscle behaviours into their forward dynamic models.

The most popular approach to modeling the aforementioned behaviours has been through the use of Hill-type muscle models (Hill, 1938; Hill, 1970; Houk, 1963; Sprigings, 1986; Caldwell, 1995). The majority of Hill models are two-component mathematical representations of muscle. The model consists of a contractile component (CC) and a series elastic component (SEC). Although there are no specific anatomical correlates to these components, the CC can be thought to represent the force producing muscles fibres, while the SEC mainly corresponds to the tendon. The SEC serves to modulate the force of the contractile component via its passive force-extension properties. As shown by Sprigings (1986), the muscle model's force output can be expressed as in Equation 1.4.2.1.

$$
F(t)=F_{m}\left(1-e^{-K t / B}\right)
$$


Where,

$$
\begin{aligned}
& \mathrm{F}_{\mathrm{m}}=\text { maximum isometric force of the represented muscle } \\
& \mathrm{K}=\text { Hookean spring stiffness } \\
& \mathrm{B}=\text { damping coefficient of viscosity } \\
& \mathrm{t}=\text { total time of maximal muscle activation }
\end{aligned}
$$

This basic two-component Hill model incorporates the activation rate property of muscle, but further additions must be made to model the additional three behaviours. Incorporating the length-tension characteristics of muscle can be accomplished by calculating $F_{m}$ as a function of resting length. Caldwell (1995) determined $F_{m}$ as a symmetrical parabolic function of muscle length. Maximum isometric force was produced when the model was at $100 \%$ of its resting length. $F_{m}$ fell to zero at both 60 and $140 \%$ of resting length. Caldwell's model was not designed to represent a specific muscle. If detailed experimental data on the force-length characteristics of a specific muscle were known, then a tailored force-length function could be formulated.

The force-velocity property of muscle can be incorporated by scaling the value of $\mathrm{F}(\mathrm{t})$ after the activation rate and force-length characteristics have been factored into the muscle model. According to methods used by Alexander (1990) in his model of human jumping, the following equation (Eq. 1.4.2.2) calculates a new value of force output $\left(\mathrm{F}(\mathrm{t})_{\text {new }}\right)$ based on the muscle's current rate of shortening.

$\mathrm{F}(\mathrm{t})_{\text {new }}=\mathrm{F}(\mathrm{t}) *\left(\left(\mathrm{~V}_{\max }-\mathrm{V}\right) /\left(\mathrm{V}_{\max }+\mathrm{G}^{*} \mathrm{~V}\right)\right)$

Eq. 1.4.2.2

Where, 


$$
\begin{aligned}
& \mathrm{V}_{\text {max }}=\text { the muscle's maximum unloaded rate of shortening } \\
& \mathrm{V} \quad=\text { the muscle's current rate of shortening } \\
& \mathrm{G} \quad=\text { a constant }
\end{aligned}
$$

Sprigings (1986) successfully demonstrated that the force enhancement phenomenon of muscle could be modeled using the two-component Hill model described above. In order to simulate force enhancement, the Hill model required that the pre-stretch of the muscle be entered as an input function. Capturing the force enhancement behaviour has also been attempted by altering the value of $F_{m}$ (Alexander, 1990). Instead of using the maximum isometric force capable of a muscle, Alexander employed the larger maximum eccentric force capability.

It has been previously stated that, for the purposes of section 1.4, the simplest methods in each of the model's development phases would be used. The most straightforward method of powering the 2D, 2-segment golfer model is through the use of constant torques. Therefore, for the conceptual model under development in this section, torque actuators will be inserted at the proximal end of each segment. The actuators will represent the sum of all muscle action across each joint. In their activated state, the actuators will produce constant values equivalent to the maximum torques possible at both the shoulder $(150 \mathrm{~N})$ and wrist $(40 \mathrm{~N})$.

\subsubsection{Develop Equations that will Govern the Motion of the Model}

It is in this phase of development that the principles of classical dynamics are imposed on the golfer model. There are only a few methods that are used to develop these equations and at the heart of each method are the basic mechanical principles. 
Generally, the equations are formulated from either an impulse-momentum framework or a work-energy perspective. Each of the methods has its advantages and disadvantages, but the questions that are asked of the model will dictate which method is best suited for the development of the equations of motion. The methods can be loosely grouped into Newton, D’Alambert, Lagrange, Hamilton, and Kane formulations.

The Newtonian method is based on creating a free-body diagram for every segment in the model and applying the standard $\Sigma \mathrm{F}=$ ma and $\Sigma \mathrm{T}=\mathrm{I} \alpha$ equations. Motion is assumed to be occurring in an inertial reference frame and the center of mass for each segment serves as the point to sum the moments. For the individual with only a basic knowledge of mechanics and mathematics, the Newtonian method is conceptually the simplest. Since each segment is dealt with individually, a deeper understanding of the system's mechanics can be garnered. However, the method compels the resolution of all inter-segmental forces whether they are desired or not. This inevitably leads to much lengthier motion equations than required if only the kinematics and external forces of the system were of interest. In an attempt to simplify the complexities of dynamics, D'Alembert's method considers the inertial terms "ma" as a force and moves it to the left side of the equation allowing a dynamic problem to be solved statically (Yamaguchi, 2001). This simplifies the situation mathematically, but results in fictitious forces and thus the potential for confusion of the underlying mechanics. However, D’Alembert's method can be applied in a non-inertial setting. This results in potentially shorter equations of motion in comparison to the Newtonian method.

An alternative to the Newtonian formulation is the Lagrangian method for developing the equations of motion. While still based on Newton's Laws of Motion, the 
Lagrangian method focuses on the total amount of physical energy in the system. The Lagrangian is defined as the difference between the kinetic and potential energy of the system (Andrews, 1995). Unlike the Newtonian method, the Lagrangian approach does not determine the inter-segmental forces, and therefore generates more compact equations of motion. However, the Lagrangian method still produces $n$ second order equations for an $n$ degree of freedom model. $\quad$ For a model comprised of as little as 4 segments moving in 3D, the second order equations become complex and difficult to solve. The Hamiltonian formulation which uses the Lagrangian formulation as its starting point overcomes this difficulty. By applying a Legendre transformation to the Lagrangian formulation, the equations of motion for an $\mathrm{n}$ degree of freedom model can be expressed by $2 n$ first order differential equations (Hogarth, 2002). This transformation results in the Hamiltonian formulation representing the generalized momenta of the system. However, if certain inter-segmental forces are desired, then the Hamiltonian approach, like its Lagrangian precursor, is not suitable.

Although researchers had a sound understanding of classical mechanics for at least a couple of centuries prior to the advent of the computer, few attempts were made at solving the equations of motions for multi-body system. When brute computational power became available, the methods previously described to develop the equations of motion were put to use. Even taking into account the considerable leaps in computing power over the past 50 years, the computational efficiency for a set of motion equations is still an issue. Kane's method for developing the equations of motion for rigid body systems greatly improves the computational efficiency of forward dynamic models (Mitiguy \& Kane, 1996). 
As a starting point, Kane's method is based on D'Alembert's reformulation of Newton's second law. Kane's method differs from the other traditional methods. According to Yamaguchi (2001),

“...Kane’s Method views the system together as a whole, creates auxiliary quantities called partial angular velocities and partial velocities, and uses them to form dot products from quantities called the generalized active forces and generalized inertia forces, which are simplified forms of the forces and moments used to write the dynamic equations of motion. It is in forming these dot products that the main advantage of Kane's Method becomes apparent, because only the forces and torques that actually create motion will survive.”

Kane's method results in $n$ first order dynamic equations describing the motions of a system with $n$ degrees of freedom. If specific inter-segmental forces and torques are of interest, then they can be determined by generating expressions for them in the same manner as the expressions for the other generalized forces. Kane's systematic mathematical treatment of Newton's laws makes his method computationally superior for use in forward dynamic applications. However, the same attributes that makes Kane’s method computationally superior, also make it conceptually more challenging than the Newtonian formulation. Therefore, for the basic conceptual purposes of this section, the equations of motion for the $2 \mathrm{D}$ golfer model will be developed using a Newtonian formulation.

The Newtonian method is initiated by determining the kinematic equations of motion for the model. These equations are also referred to as constraint equations, as 
they dictate the configuration of the system at any point in time. The nature of our system (a planar double pendulum fixed at one end) suggests that the angular orientations of the each segment $\left(\theta_{1}\right.$ and $\left.\theta_{2}\right)$ should serve as the two configuration variables that define the orientation of the model (Fig. 1.4.3.1).

The position of the mass centers of the arm (A) and club (B) can be determined from the segment lengths, the distances from the proximal joint centers to the centers of mass of each segment, and the angle each segment makes with the positive $\mathrm{X}$-axis. For each segment, the center of mass positions can be represented by separate expressions for both their $\mathrm{X}$ and $\mathrm{Y}$ coordinates.

$$
\begin{array}{ll}
\mathbf{p}_{\mathrm{x}}{ }^{\mathrm{OA}}=\mathrm{r}_{1} \cos \left(\theta_{1}\right) & \text { Eq. 1.4.3.1 } \\
\mathbf{p}_{\mathrm{y}}{ }^{\mathrm{OA}}=\mathrm{r}_{1} \sin \left(\theta_{1}\right) & \text { Eq. 1.4.3.2 } \\
\mathbf{p}_{\mathrm{x}}{ }^{\mathrm{OB}}=\mathrm{L}_{1} \cos \left(\theta_{1}\right)+\mathrm{r} 3 \cos \left(\theta_{2}\right) & \text { Eq. 1.4.3.3 } \\
\mathbf{p}_{\mathrm{y}}{ }^{\mathrm{OB}}=\mathrm{L}_{1} \sin \left(\theta_{1}\right)+\mathrm{r} 3 \sin \left(\theta_{2}\right) & \text { Eq. 1.4.3.4 }
\end{array}
$$

Where $\mathbf{p}_{\mathrm{x}}{ }^{\mathrm{OA}}$ stands for the $\mathrm{X}$ component of the position vector from point $\mathrm{O}$ to point $\mathrm{A}$. 


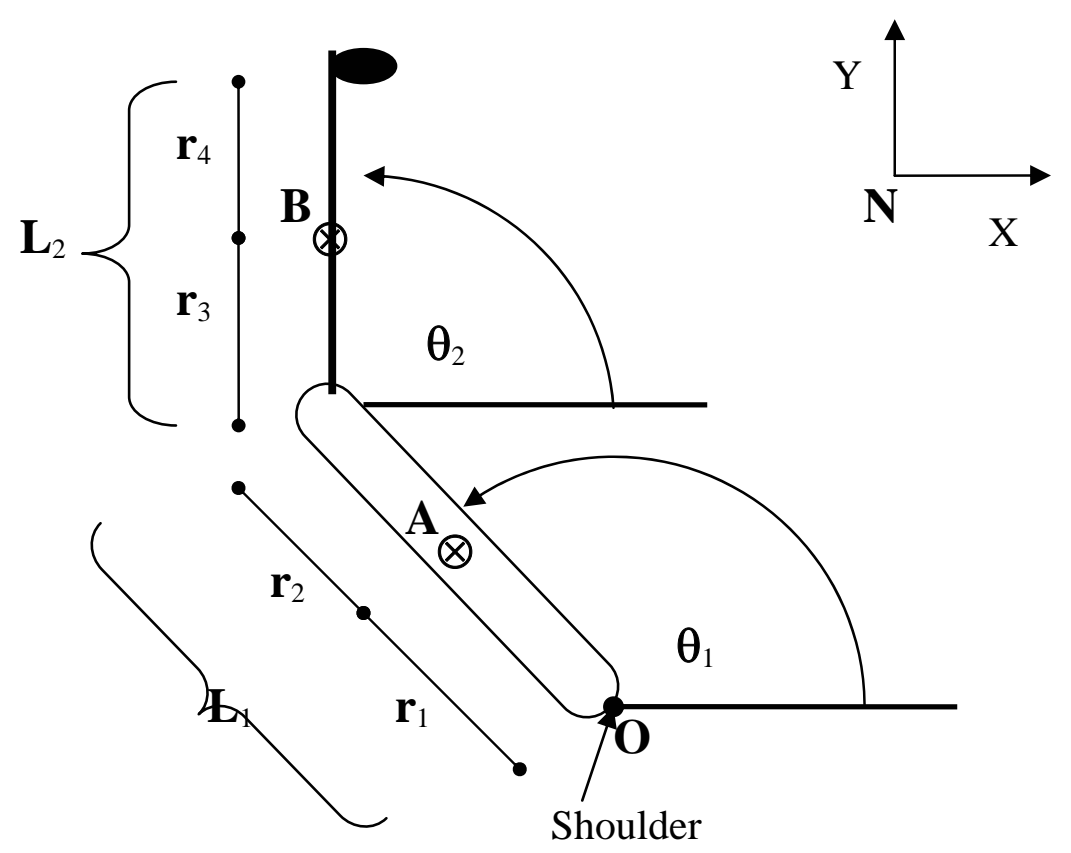

Figure 1.4.3.1 Schematic of two-segment golfer model.

Taking the second derivative of each position vector with respect to time yields the following equations for the linear accelerations of the centers of mass for each segment in the inertial reference frame $\mathrm{N}$. These are the kinematical equations for the model.

$$
\begin{aligned}
& \mathrm{N}_{\mathbf{a}_{\mathrm{x}}}^{\mathrm{A}}=-\alpha_{1} \mathrm{r}_{1} \sin \left(\theta_{1}\right)-\omega_{1}^{2} \mathrm{r}_{1} \cos \left(\theta_{1}\right) \\
& \mathrm{N}_{\mathbf{a}_{\mathrm{y}}}^{\mathrm{A}}=\alpha_{1} \mathrm{r}_{1} \cos \left(\theta_{1}\right)-\omega_{1}^{2} \mathrm{r}_{1} \sin \left(\theta_{1}\right) \\
& \mathrm{N}_{\mathbf{a}_{\mathrm{x}}}{ }^{\mathrm{B}}=-\alpha_{1} \mathrm{~L}_{1} \sin \left(\theta_{1}\right)-\omega_{1}{ }^{2} \mathrm{~L}_{1} \cos \left(\theta_{1}\right)-\alpha_{2} \mathrm{r}_{3} \sin \left(\theta_{2}\right)-\omega_{2}{ }^{2} \mathrm{r}_{3} \cos \left(\theta_{2}\right) \quad \text { Eq. 1.4.3.7 } \\
& \mathrm{N}_{\mathbf{a}_{\mathrm{y}}}{ }^{\mathrm{B}}=\alpha_{1} \mathrm{~L}_{1} \cos \left(\theta_{1}\right)-\omega_{1}{ }^{2} \mathrm{~L}_{1} \sin \left(\theta_{1}\right)+\alpha_{2} \mathrm{r}_{3} \cos \left(\theta_{2}\right)-\omega_{2}{ }^{2} \mathrm{r}_{3} \sin \left(\theta_{2}\right) \quad \text { Eq. 1.4.3.8 } \\
& \text { Where, } \\
& { }^{\mathrm{N}} \mathbf{a}_{\mathrm{x}}{ }^{\mathrm{A}} \text { stands for the acceleration of } \mathrm{A} \text { in the } \mathrm{X} \text { direction with respect to } \mathrm{N} \\
& \omega_{1} \text { is the angular velocity of segment } 1 \text { (arm) with respect to } \mathrm{N} \\
& \alpha_{1} \text { is the angular acceleration of segment } 1 \text { (arm) with respect to } \mathrm{N}
\end{aligned}
$$


The next step is to derive equations representing the sum of the forces, and the sum of the moments acting on each segment in the model. Constructing separate freebody diagrams for each segment in the model is essential for correctly deriving the equations (Fig. 1.4.3.2)

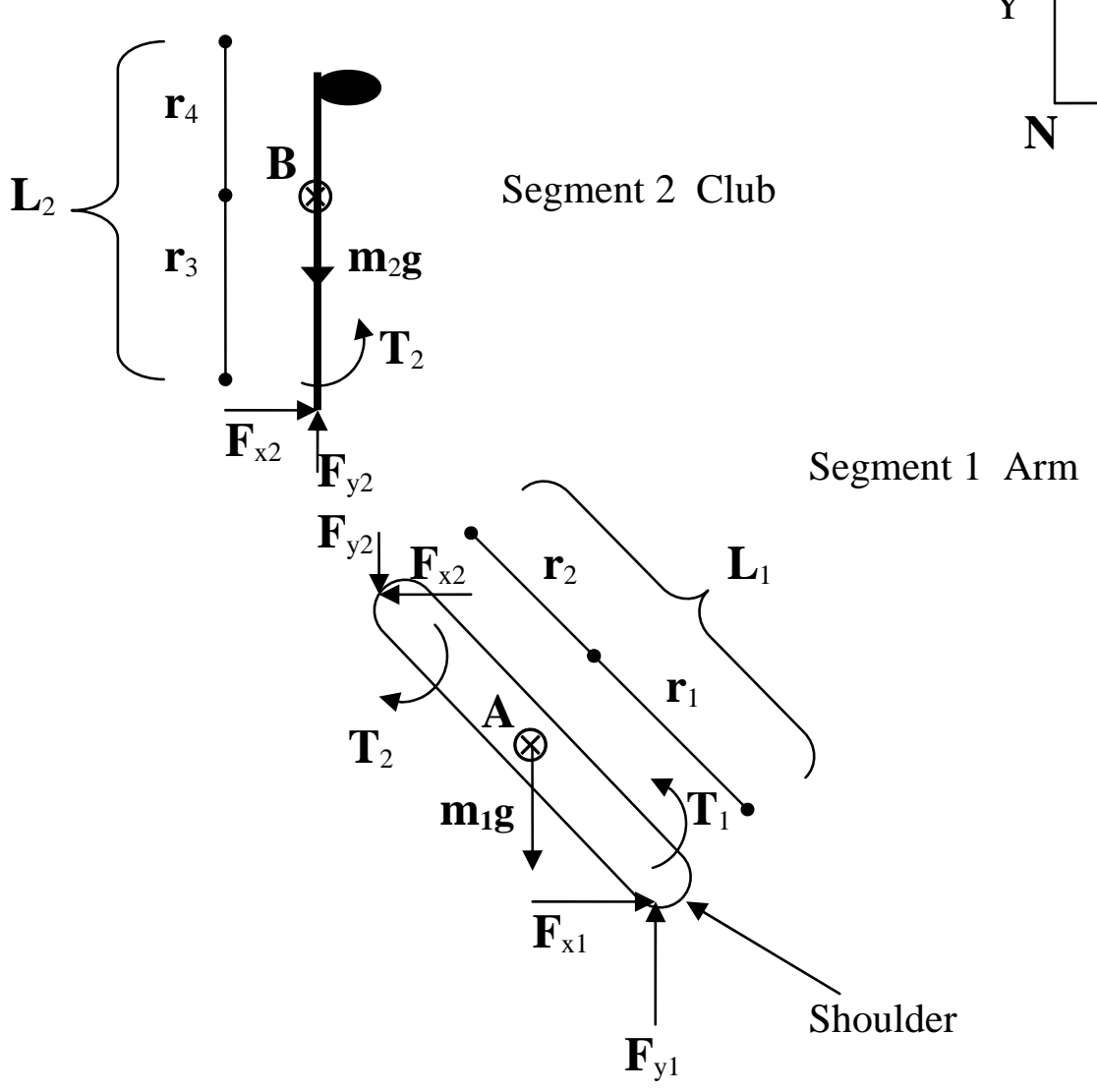

Figure 1.4.3.2 Free-body diagram of two-segment golfer model.

Each segment will have three kinetic equations; one for the sum of the forces in the $\mathrm{X}$ direction, one for the sum of the forces in the $\mathrm{Y}$ direction, and one for the sum of 
the moments about the center of mass of the segment. These equations are presented in a general form suitable for implementation into a computer program.

For segment 1,

$$
\begin{aligned}
& \mathbf{F}_{\mathrm{x} 1}-\mathbf{F}_{\mathrm{x} 2}=\mathrm{m}_{1}{ }^{\mathrm{N}} \mathbf{a}_{\mathrm{x}}{ }^{\mathrm{A}} \\
& \mathbf{F}_{\mathrm{y} 1}-\mathbf{F}_{\mathrm{y} 2}-\mathrm{m}_{1} \mathrm{~g}=\mathrm{m}_{1}{ }^{\mathrm{N}} \mathbf{a}_{\mathrm{y}}{ }^{\mathrm{A}} \\
& \Sigma \mathrm{M}_{\mathrm{A}}=\mathbf{F}_{\mathrm{x} 1} \mathrm{r}_{1} \sin \left(\theta_{1}\right)-\mathbf{F}_{\mathrm{y} 1} \mathrm{r}_{1} \cos \left(\theta_{1}\right)+\mathbf{F}_{\mathrm{x} 2} \mathrm{r}_{2} \sin \left(\theta_{1}\right) \\
& \quad-\mathbf{F}_{\mathrm{y} 2} \mathrm{r}_{2} \cos \left(\theta_{1}\right)+\mathbf{T}_{1}-\mathbf{T}_{2}=\mathrm{I}_{1} \alpha_{1}
\end{aligned}
$$

For segment 2,

$$
\begin{aligned}
& \mathbf{F}_{\mathrm{x} 2}=\mathrm{m}_{2}{ }^{\mathrm{N}} \mathbf{a}_{\mathrm{x}}{ }^{\mathrm{B}} \\
& \mathbf{F}_{\mathrm{y} 2}-\mathrm{m}_{2} \mathrm{~g}=\mathrm{m}_{2}{ }^{\mathrm{N}} \mathbf{a}_{\mathrm{y}}{ }^{\mathrm{B}} \\
& \Sigma \mathrm{M}_{\mathrm{B}}=\mathbf{F}_{\mathrm{x} 2} \mathrm{r}_{3} \sin \left(\theta_{2}\right)-\mathbf{F}_{\mathrm{y} 2} \mathrm{r}_{3} \cos \left(\theta_{2}\right)+\mathbf{T}_{2}=\mathrm{I}_{2} \alpha_{2}
\end{aligned}
$$

The kinetic equations can be combined with the kinematic constraint equations allowing for separate expressions for the angular acceleration of each segment to be determined. Usually, a mathematical software package such as Mathematica ${ }^{\circledR}$ or Maple $^{\odot}$ is employed to generate the relatively lengthy closed-form expressions for the angular accelerations in terms of $\theta_{1}, \theta_{2}, \omega_{1}, \omega_{2}, T_{1}$, and $T_{2}$. All other variables enter the expression as known quantities. An expression for the segment acceleration permits the calculation of the segment velocity and position, through the process of numerical integration. 


\subsubsection{Determine How the Equations will be Solved}

For most musculoskeletal models, the equations of motion are a set of second order differential equations that need to be solved numerically. This is an iterative process that solves for the corresponding first and zeroth order equations of motion at each time step. The most straightforward method of numerically solving differential equations is known as Euler's method. The following is a description of its implementation with the 2D model.

Given the chance, muscle torques and gravity will act on the golfer model resulting in acceleration. In the previous section, expressions were developed for the angular accelerations of each segment in the model. It is possible to determine angular acceleration for each segment at $\mathrm{t}=0$ given initial position and velocity data. The total simulation time for our model, from initiation of movement until impact with the ball, can be divided up into a series of smaller time intervals. Assuming that the accelerations determined at $\mathrm{t}=0$ remain constant during the entire first time interval, Euler's method can be used to determine both velocity and displacement at the end of this interval. These new velocity and displacement values along with new muscle torques are entered into the equations of motion to determine accelerations for the next time interval. The following two equations demonstrate how the angular kinematics are stepped through in a sequential manner.

$$
\begin{aligned}
& \omega_{\mathrm{I}+1}=\omega_{\mathrm{I}}+\alpha_{\mathrm{I}} \Delta \mathrm{t} \\
& \theta_{\mathrm{I}+1}=\theta_{\mathrm{I}}+\omega_{\mathrm{I}} \Delta \mathrm{t}
\end{aligned}
$$

Where,

I indicates the present time interval 
I +1 indicates the next time interval

$\theta$ is the angular position

$\omega$ is the angular velocity

$\alpha$ is the angular acceleration

$\Delta \mathrm{t}$ is the length of the time interval

This procedure is repeated until a predetermined condition is reached signifying the end of the simulation such as contact with the ball.

The extension from Euler's method to other forms of numerical solution is one of technical sophistication not conception. Two commonly used numerical solution methods in forward dynamic models are versions of the Runge-Kutta and Kutta-Merson algorithms. Remember that the Euler method assumed that the acceleration determined at the start of a time interval remained constant for the duration of that time interval. This assumption allows the motion equations to be solved a single time for each time interval. While computationally efficient, it can lead to large errors due to poor approximation of the 'true' acceleration curve. That is, the acceleration curve that would be generated if infinitely small time intervals were used. Kutta methods attempt to evaluate how much the acceleration function is changing during the time interval. This is accomplished by evaluating the motion equations at several points over the time interval and using an average acceleration. Advanced Kutta algorithms use variable time intervals throughout the simulation. For example, assume the acceleration function remained constant for the first half of the simulation and became erratic over the second half of the simulation. Advanced algorithms would employ large time intervals over the 
first half and very small time intervals over the second half. This allows a balance between computational efficiency and accuracy.

The forward dynamics model is now functional. Given specific inputs from the muscle torque generators the behaviour of the model can be predicted. However, the goal of the model was to determine the muscle coordination strategy a golfer should implement to achieve maximum clubhead velocity at impact with the ball. This requires knowing when to activate the muscle torques and if necessary when to deactivate them. This knowledge can be gained by optimizing the forward dynamics model.

\subsubsection{Implement an Optimization Scheme}

Optimization entails maximizing or minimizing the value of a function by searching for the best combination of that function's independent variables. In the context of our research question from section 1.4, clubhead velocity at impact is the function whose value needs to be maximized. Clubhead velocity at impact is determined by the muscle control strategy used at the shoulder and wrist during the downswing. Specifically, the start and duration times for the torque generators at the shoulder and wrist are the independent or control variables for the optimization. There exists an optimal pattern of torque generator activation that will lead to maximum clubhead velocity at impact with the ball. A scheme needs to be employed that will search for and return the values of the control variables that will generate the optimal pattern of torque generator activation. It is possible to conduct an optimization by either maximizing or minimizing a function's value. Since we are interested in larger 
clubhead speeds, optimization approaches will only be discussed in the context of maximizing a function.

To help acquire an understanding of optimization methods, let's temporarily consider a simplified scenario under which the golfer model requires optimization. Assume that the time of onset of the shoulder torque requires no control and is fully activated throughout the entire simulation. Further, assume that once the wrist torque is activated, it remains active for the duration of the simulation. This leaves only a single control variable; the start time for the wrist torque. In this simplified state, clubhead velocity is only a function of wrist torque start time. In terms of optimization, this is considered a one-dimensional search space. A hypothetical graph of clubhead velocity at impact as a function of wrist torque start times is shown in Fig. 1.4.5.1. With only a single control variable it is easy to visualize the search space. The goal of the optimization algorithm is to find, as quickly as possible the wrist torque start time associated with the largest clubhead velocity which is represented by the global maximum. 


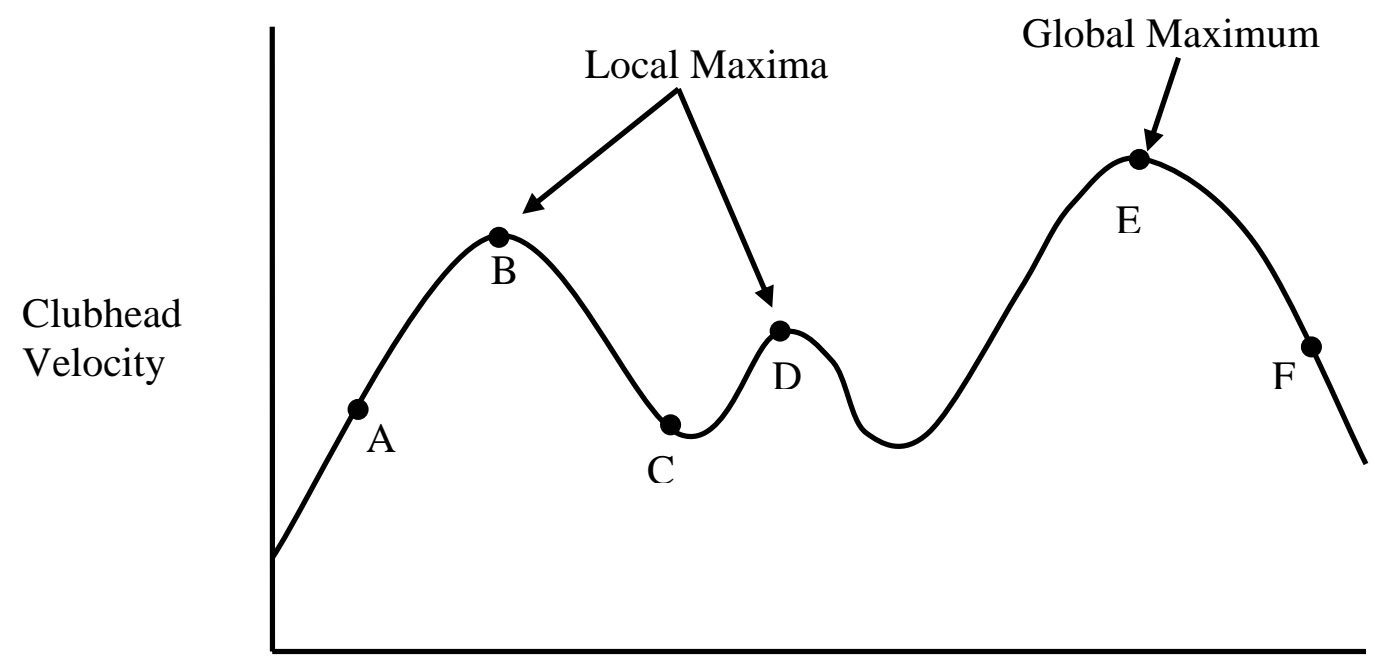

Wrist Torque Start Time

Figure 1.4.5.1 Search space for a single variable optimization problem.

The following overly generalized categories of optimization are not based on their underlying mathematical implementations but rather on what they are intending to accomplish conceptually.

Perhaps the most obvious way of finding the global maximum of a function is to evaluate the function at all possible locations. This is referred to as an exhaustive search. This is truly the only method that will provide, with absolute certainly, the global maximum. However, this is also the method that will require the most computation time, and in most cases, this computation time is far greater than is feasible. It would be beneficial if our optimization scheme had a better way to move around the search space and perhaps determine if it reached a maximum before having to evaluate all possible locations. 
To save on computational time a local search method could be used. The general schema for a local search algorithm is as follows. Randomly pick a point in the search space from which to start and evaluate the function at that point. Perform some transformation to the current point's coordinates, so that a new point nearby is selected for evaluation. If the new point has a higher function value, then the new point becomes the current point. If the new point has a lower function value, then another new point is selected based on another transformation of the current point's coordinates. This pattern is repeated until no improvements in the function's value can be found from the search's current position. When this happens, the function's value at the current position becomes the maximum returned by the optimization algorithm. Local searches, such as the hill-climbing method just described, differ only in how the transformation is applied to the current position. The problem with hill-climbers and local searches in general, is that they tend to get trapped on local maximums. Consider the diagram in Fig. 1.4.5.1, if the search started at point A, then the algorithm would find its way to B and realize that all nearby points result in smaller function values and conclude that B is the optimal solution. If the starting point was $\mathrm{C}$, then the search would likely conclude at either B or D. As you can see, the success of a local search depends on the initial starting point. If the search started at F, then the algorithm would have serendipitously found the global maximum at E. This dependency on an initial starting point requires that local search algorithms must be started several times from random locations in the search space to have a chance at finding the global maximum. Essentially, the downfall of local searches is that they don't have the ability to climb down from relatively short hills. 
The first method to have improved success at finding a global optimum during a single run was simulated annealing. Simulated annealing can be thought of as a local search method with a twist. The twist is the ability for the method to climb down from local maximums and continue to look for higher peaks. It can climb down because its choice of whether or not to move to a new point is probabilistic in nature. The hillclimber moved to a new point if it was better, if the new point wasn't better, then it maintained its current position and evaluated another new point for comparison. Eventually if it stayed in the same spot long enough, the optimization would conclude. This would happen if it was at the top of a hill, but not necessarily a global maximum. When the simulated annealing algorithm evaluates a new position, it will move to that position if it is better, but there is a chance it will move to that new position even if it results in a lower function value. This probability is based on a parameter, $\mathrm{T}$, and on how much lower the function value is at the new position compared to the current one. The probability that a poorer position will be accepted is determined by the following equation (Eq. 1.4.5.1).

$$
\text { Probabilty }=\frac{1}{1+\mathrm{e}^{\text {(current function value - new function value }) / \mathrm{T}}}
$$

Notice that if the function value at the current position equals that of the new position then there is a $50 \%$ chance that either position will be selected. If the new position has a much lower function value than the current position, then the chance of the new position being selected as the current position is low. The parameter $\mathrm{T}$ signifies why this type of optimization is referred to as simulated annealing which hails from the field of thermodynamics. 
To grow a crystal, you want to start with the material in a very hot liquid state and slowly cool the substance until it becomes locked into a crystal form. Problems occur if the liquid is cooled to quickly. If cooled quickly, the crystal will have irregularities and the energy level is higher than a perfectly ordered crystal. The control parameter T signifies the rate at which the solution to the optimization is "cooled". The variable $\mathrm{T}$ essentially controls the ability of the algorithm to climb down from peaks. At the start of the optimization, $\mathrm{T}$ is large and the simulated annealing algorithm behaves like a random search, as the temperature cools ( $\mathrm{T}$ decreases in value) new points that are worse than the current point have less chance on being selected. Near the end of the optimization, the simulated annealing algorithm behaves like a regular hill-climber, but hopefully, the previous iterations have led it to the hill with the highest peak.

The three optimization methods discussed thus far evaluate a single solution to the optimization during each iteration. Optimization methods have been developed that allow a population of solutions to be evaluated at each step during the optimization. This branch of optimization has been referred to as genetic or evolutionary programming. From the initial statements in this paragraph, it might appear as though an evolutionary program could simply be several hill-climbers, or simulated annealing programs running at the same time. This would be no more successful than repeating the optimization scheme several times, and accepting the best answer found. However, evolutionary programs actually have the solutions competing and interacting with each other during every iteration of the optimization. Based on Darwin's theories of evolution, solutions compete against each other and the fittest individuals have the best chance of surviving to pass on their attributes to future generations. In addition, just like 
in natural evolution, a random mutation component can be added to the solutions that survive into the next generation (iteration). Suppose that 6 points (each point represents an individual) were randomly picked for evaluation from the search space shown in Figure 1.4.5.1. After evaluation each individual was given a chance on being selected to survive into the next iteration (generation). The magnitude of the probability of being selected is directly proportional to their fitness (function value at that point) relative to all the other individuals. For example, if Individual $\# 1$ had a fitness of $45 \mathrm{~m} / \mathrm{s}$, and the sum of all six individuals' fitness values was $90 \mathrm{~m} / \mathrm{s}$, then Individual \#1 would have a 50 \% (45/90) chance of being selected. Each individual is given a slot on a roulette wheel whose area is proportional to their relative fitness, i.e. Individual \#1's area occupies $50 \%$ of the roulette wheel. In selecting 6 individuals that will survive into the next generation, the roulette wheel is spun 6 times. If the die lands in a particular individual's space, then that individual survives. This system allows really fit individuals to be selected more than once. Once selected, their genetic code (the control variables), or in this case wrist torque start time is mutated, and these mutated individuals are evaluated in the next generation.

An evolutionary optimization scheme was employed in the development and implementation of the model used in this thesis. Since evolutionary programming is conceptually straightforward, a more detailed discussion on the program's structure will be saved until the next chapter.

This concludes the general explanation and review of forward dynamic simulations. The description of the methods that were used in the development of the golfer model used in this thesis will describe only the finer points of the methods and 
not how the methods actually work or were employed. The reader should refer back to this chapter for conceptual insight into the specific workings of an optimized forward dynamics model. 
2

METHODS 


\subsection{Mathematical Model}

\subsubsection{General Description of Golfer-Club Model}

A representative mathematical model of a golfer was constructed using a sixsegment (torso, arm, and four club segments), 3D, linked system (Fig. 2.1.1.1). Based on the hypotheses of this thesis, the purpose of the golfer portion of the model was to generate realistic kinetic profiles on the club segment. It was decided that the exclusion of additional segments from the model would not prevent the golfer portion of the model from fulfilling its purpose. An in depth discussion on this topic is provided in sections 4.5 and 4.8 of this thesis.

The torso segment was constrained to rotate in a plane angled at $30^{\circ}$ above the horizontal. The arm segment was constrained to move in a plane that was angled at $50^{\circ}$ above the horizontal (Fig. 2.1.1.2). These values were chosen based on static measurements taken from golf professionals featured in Golf Digest Magazine. An alternative to constraining the segments to move in planes would have been to use control torques. However, this would have made the optimization process needlessly more complex. In addition, it would have been very difficult to compare results between separate simulations that featured varying swing planes. Due to the high variability in swing plane among golfers (Williams \& Sih, 2002), choosing a representative swing plane within this range seems to be a reasonable approach. Further, the validity of this assumption was checked through comparison to the swing planes measured by Coleman and Rankin (2005). 


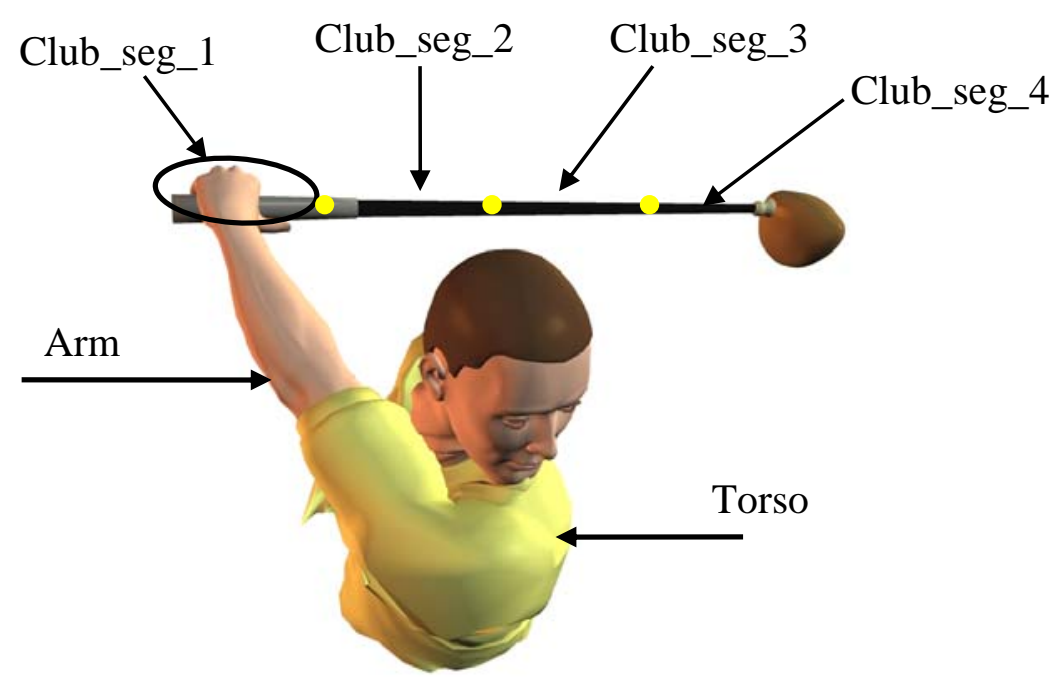

Figure 2.1.1.1 Six segment 3D golfer-club model.

The torso segment was modelled as a rigid body representing the entire torso rotating about its longitudinal axis. The arm segment was modelled as a rigid body encompassing both the upper arm and forearm with the elbow angle fixed at $180^{\circ}$. In addition to moving in a plane, the arm segment was free to rotate about its longitudinal axis representing both internal-external rotation at the shoulder and supination-pronation at the elbow. The golf club was modelled as four rigid segments with the proximal end 
representing both the grip end of the club and hand of the golfer. The club segments were connected by spring-damper elements that allowed the shaft to deflect in both the lead/lag and toe up/toe down directions. The most proximal club segment, connected to the arm, was constrained to have motion only in a representative adduction-abduction plane relative to the arm segment. This is in agreement with anatomical constraints that prevent rotation at the wrist about the longitudinal axis of the forearm and a proper golf swing in which the lead wrist is kept in an approximately neutral position with regards to flexion and extension. The initial configuration of the golfer-club model for every simulation was as depicted in Figure 2.1.1.1. An initial angular velocity of $5 \mathrm{rad} / \mathrm{s}$, in the backswing direction, about the torso axis was also given to all segments of the model, so as to simulate the dynamic transition from the backswing into the downswing. 


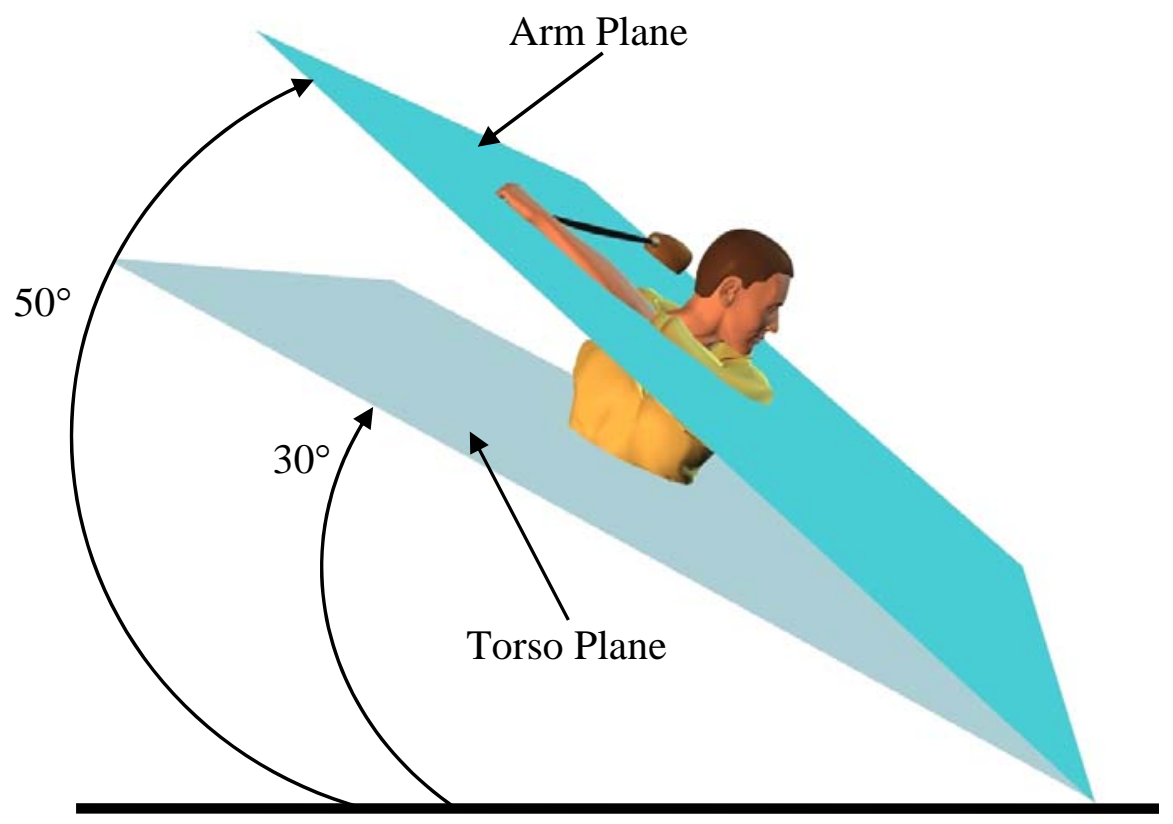

Figure 2.1.1.2 The torso segment and arm segment were constrained to rotate in specific planes.

Four torque generators that adhered to the activation rates and force-velocity properties of human muscle were inserted at the proximal end of each segment, and provided the model with the capability of controlling energy to the system (Springing \& Neal, 2000). The force-length property of muscle was not incorporated into the model as it was expected to play a second order role (Caldwell, 1995). This will be discussed in more detail in section 4.5. One torque generator produced rotation of the torso segment (torso torque generator), a second produced extension/abduction of the arm (shoulder torque generator), a third created rotation about the longitudinal axis of the 
arm (arm rotation torque generator), and a fourth produced adduction of the hand-club segment (wrist torque generator).

Parameter values for segment length, moment of inertia, and mass for a representative golfer with a body mass of $80 \mathrm{~kg}$, and a standing height of $1.83 \mathrm{~m}$, were calculated using the regression equations provided by Zatsiorsky (2002). Parameter values for the club segments were based on taking direct measurements of a standard driver designed in 2001. Moment of inertia values for the shaft segments were determined by modelling the segments as hollow cylinders (Table 2.1.1.1). The moment of inertia values for the clubhead were determined geometrically by modelling the head as a semi-ellipsoid (Appendix B). Providing Autolev ${ }^{\circledR}$ with the moments of inertia about the three principal axes, of those segments not constrained to move in a plane, allowed Autolev ${ }^{\odot}$ to generate the full inertia tensors necessary for the 3D dynamical equations.

Table 2.1.1.1 Six-segment golfer-club model segment parameters

\begin{tabular}{|l|l|l|l|l|l|l|l|l|}
\hline Segment & $\begin{array}{l}\text { Mass } \\
(\mathrm{kg})\end{array}$ & $\begin{array}{l}\text { Length } \\
(\mathrm{cm})\end{array}$ & $\begin{array}{l}\text { CM_x } \\
(\mathrm{cm})\end{array}$ & $\begin{array}{l}\text { CM_y } \\
(\mathrm{cm})\end{array}$ & $\begin{array}{l}\text { CM_z } \\
(\mathrm{cm})\end{array}$ & $\begin{array}{c}\text { I_x } \\
\left(\mathrm{kg}^{*} \mathrm{~cm}^{2}\right)\end{array}$ & $\begin{array}{c}\text { I_y } \\
\left(\mathrm{kg}^{*} \mathrm{~cm}^{2}\right)\end{array}$ & $\begin{array}{c}\text { I_z } \\
\left(\mathrm{kg}^{*} \mathrm{~cm}^{2}\right)\end{array}$ \\
\hline Torso & 34.61 & 40.0 & - & 20.0 & - & - & - & 3655 \\
\hline Arm & 3.431 & 60.0 & 0.0 & 0.0 & 26.1 & 1076 & 1096 & 58.06 \\
\hline Club_seg_1 & 0.534 & 30.0 & 0.0 & 0.0 & 5.6 & 11.81 & 11.81 & 6.287 \\
\hline Club_seg_2 & 0.021 & 30.0 & 0.0 & 0.0 & 14.9 & 1.579 & 1.579 & 0.009 \\
\hline Club_seg_3 & 0.020 & 30.0 & 0.0 & 0.0 & 14.9 & 1.509 & 1.509 & 0.005 \\
\hline
\end{tabular}


Three separate golfer-club models were developed. They differed only with regards to the maximum torque outputs and maximum speeds of contraction capable from the four torque generators. This allowed the role of shaft flexibility to be evaluated over a range of swing speeds. In this thesis, swing speed refers to the potential of the golfer to generate clubhead speed. The model representing the slowest swing speed capabilities will be referred to as Golfer-Slow, the model representing mid swing speed capabilities will be referred to as Golfer-Medium, and the model with the capability for

the highest swing speeds will be known as Golfer-Fast. The word capability is used because these models must still be optimized with regards to the timing of their torque generators to determine exactly how much swing speed they can generate.

\subsubsection{Method for Measuring Shaft Deflection}

The magnitude of shaft deflection used in this thesis was calculated in the following manner. Consider a golf club broken into segments defined by points along its length. The two most proximal points are at the top and bottom of the grip, while the most distal point is at the position of the center of mass of the clubhead along the $z$ axis of the shaft. It was assumed that minimal bending occurred between the two most proximal points on the shaft. As such, a vector (V1) representing the position of a rigid shaft was constructed using the coordinates from the two most proximal markers. A unit vector (V2) perpendicular to the rigid shaft vector was also formed along the $y$ axis. A third vector (V3) was defined from the most proximal point, to the most distal point. A measurement of lead/lag deflection was found by taking the dot product of V2 and V3 (Fig. 2.1.2.1). A similar process was used for calculating toe-up/toe-down deflections. 
However, for toe-up/toe-down values, the V2 vector was formed along the $x$ axis instead of the $y$ axis.

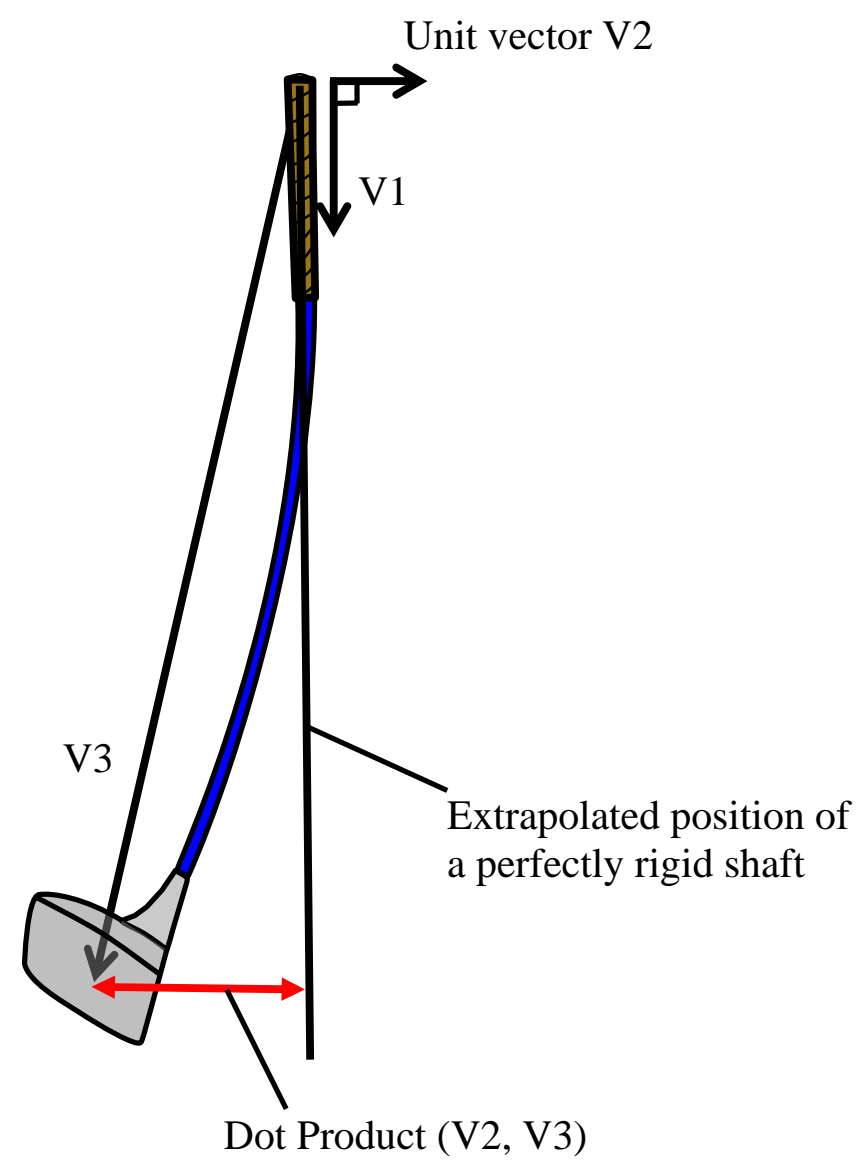

Figure 2.1.2.1 Calculation of lead/lag shaft deflection along the $y$ axis of the shaft. Identical methods were used for calculating toe-up/toe-down deflection along the $x$ axis of the shaft.

\subsubsection{Determining Shaft Stiffness and Damping Parameters}

The four segments of the modelled club were connected by three rotational spring-damper elements. Separate stiffness constants for each spring were 
experimentally determined so that three shafts of varying stiffness could be represented in the model.

Three drivers identical in all physical aspects with the exception of shaft stiffness were used to create three separate simulated clubs for use in the golfer-club model. According to the shaft specifications put forth by the manufacturer, the shafts represented three level of stiffness: flexible, regular, and stiff. The following procedure was used to determine the three stiffness constants for each of the three clubs. Each club was rigidly fixed at its grip end up to a point $30 \mathrm{~cm}$ down the shaft. This simulated the modelled club which was perfectly rigid for the first $30 \mathrm{~cm}$. Markers were placed on the shaft according to the segments defined by the mathematical club model. Specifically, in addition to a marker at the top of the grip, and a marker on the hozel, markers were located at the same positions as the spring elements in the modelled clubs. This partitioned the club into four segments (Fig. 2.1.3.1). A $1 \mathrm{~kg}$ weight was suspended from the hozel resulting in shaft deflection. The deflection was video recorded and the coordinates of the markers were determined using the motional analysis software package HU-M-AN ${ }^{\odot}$. From these coordinates, the relative angles between adjacent links were determined. This procedure resulted in three sets of three angles, with each set of angles representing a different shaft. 


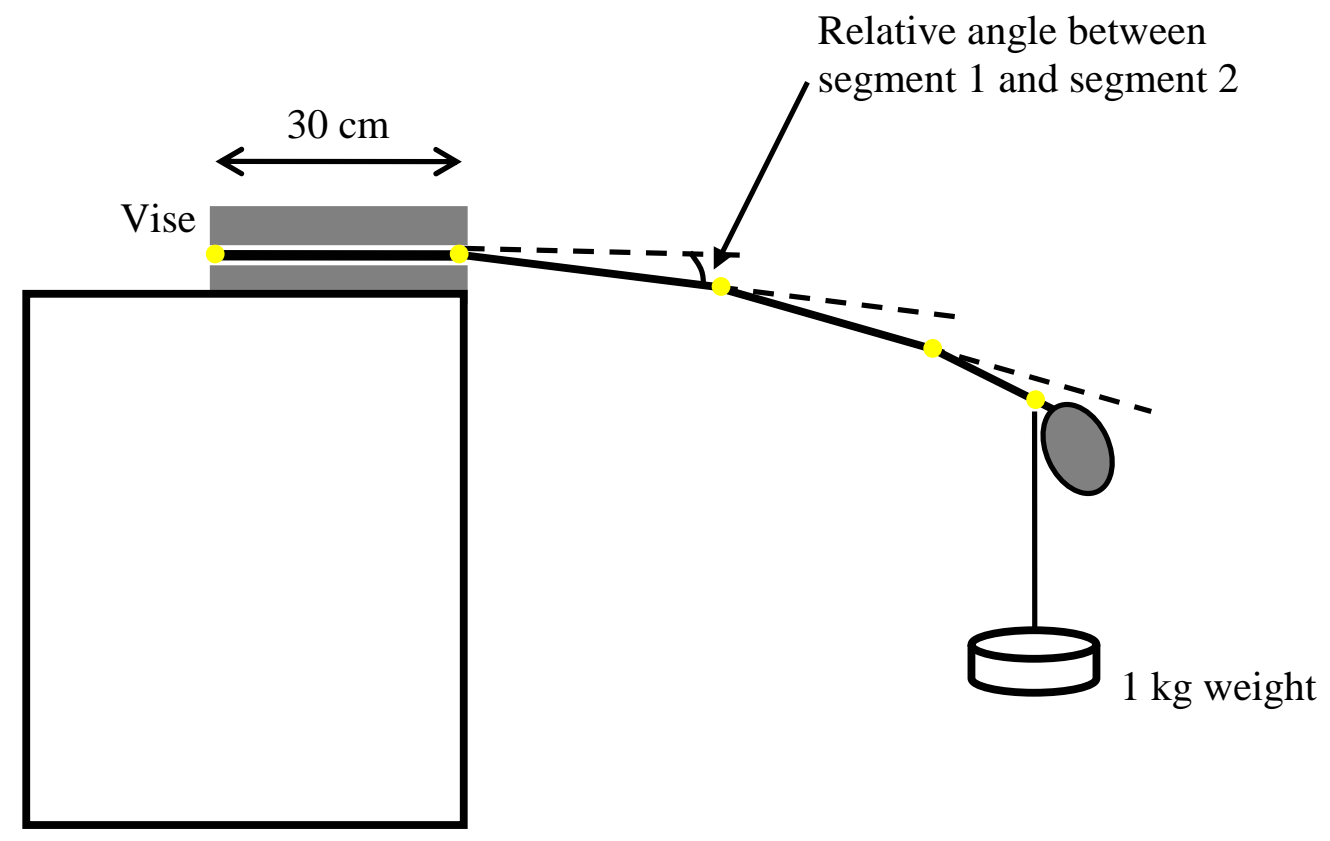

Figure 2.1.3.1 Experimental set-up for determining shaft stiffness.

This experimental setup was simulated using the mathematical model of the club. Using an optimization scheme similar to the one described below in section 2.2, stiffness constants for each of the three spring elements were adjusted until the relative angles between segments in the mathematical model matched a set of relative angles determined experimentally. This procedure was repeated for each of the three clubs measured experimentally resulting in three sets of three stiffness parameters (Table 2.1.3.1). Each set of stiffness parameters then represented a specific club with a certain shaft stiffness. The most flexible club will be referred to as Club-Flexible, the regular flex club will be known as Club-Regular, and the stiffest club will be referred to as Club-Stiff. 
Table 2.1.3.1 Stiffness coefficients for rotational springs of simulated drivers

\begin{tabular}{|l|c|c|c|}
\hline & Club-Flexible (Nm/rad) & Club-Regular (Nm/rad) & Club-Stiff (Nm/rad) \\
\hline Spring 1 & 501 & 536 & 625 \\
\hline Spring 2 & 135 & 159 & 194 \\
\hline Spring 3 & 81 & 87 & 148 \\
\hline
\end{tabular}

The damping coefficient for the rotational spring-damper elements could not be experimentally determined. Determining a damping coefficient experimentally, would require that the club be gripped by a golfer's hands and put into an oscillation. Unfortunately, a golfer cannot simulate the grip characteristics that are applied during the swing in the type of experimental set-up that would be required to determine a damping coefficient. Therefore, a level of damping was chosen that resulted in the best agreement between simulation and live golfer testing results. The damping coefficient that provided the best level of agreement was $10(\mathrm{Nm}) /(\mathrm{rad} / \mathrm{s})$. This meant for a given axis of rotation, a rotational spring-damper element took the form:

$$
\text { Torque }=(-\mathrm{K} \theta)-(\mathrm{C} \omega)
$$

Where,

$\mathrm{K}$ is the stiffness coefficient

$\theta$ is the relative angular displacement of the distal segment

$\mathrm{C}$ is the damping coefficient

$\omega$ is the relative angular velocity of the distal segment 


\subsubsection{Equations of Motion}

The equations governing the motion of the mathematical model were based on Newton's Laws of Motion and were formulated according to Kane's method (Yamaguchi, 2001). The software program Autolev ${ }^{\odot}$ was used to facilitate the generation of the 3D dynamical equations into FORTRAN ${ }^{\odot}$ programming code. To solve the set of first order differential equations determining the motion of the system, numerical integration was performed using a modified Kutta-Merson algorithm provided by Autolev ${ }^{\odot}$. The Autolev ${ }^{\odot}$ code is provided in Appendix C.

\subsubsection{Generalized Coordinates of the Golfer Model}

The following section was included to supply the reader with information that would facilitate replication of the mathematical model used in this thesis. This information is intended to be used in conjunction with the Autolev ${ }^{\odot}$ code provided in Appendix C. Knowledge of the following material is not necessary for comprehending the results presented in this thesis; rather it simply offers a connection to the Autolev ${ }^{\odot}$ code. For those not familiar with Autolev ${ }^{\odot}$, proceed to section 2.2. 

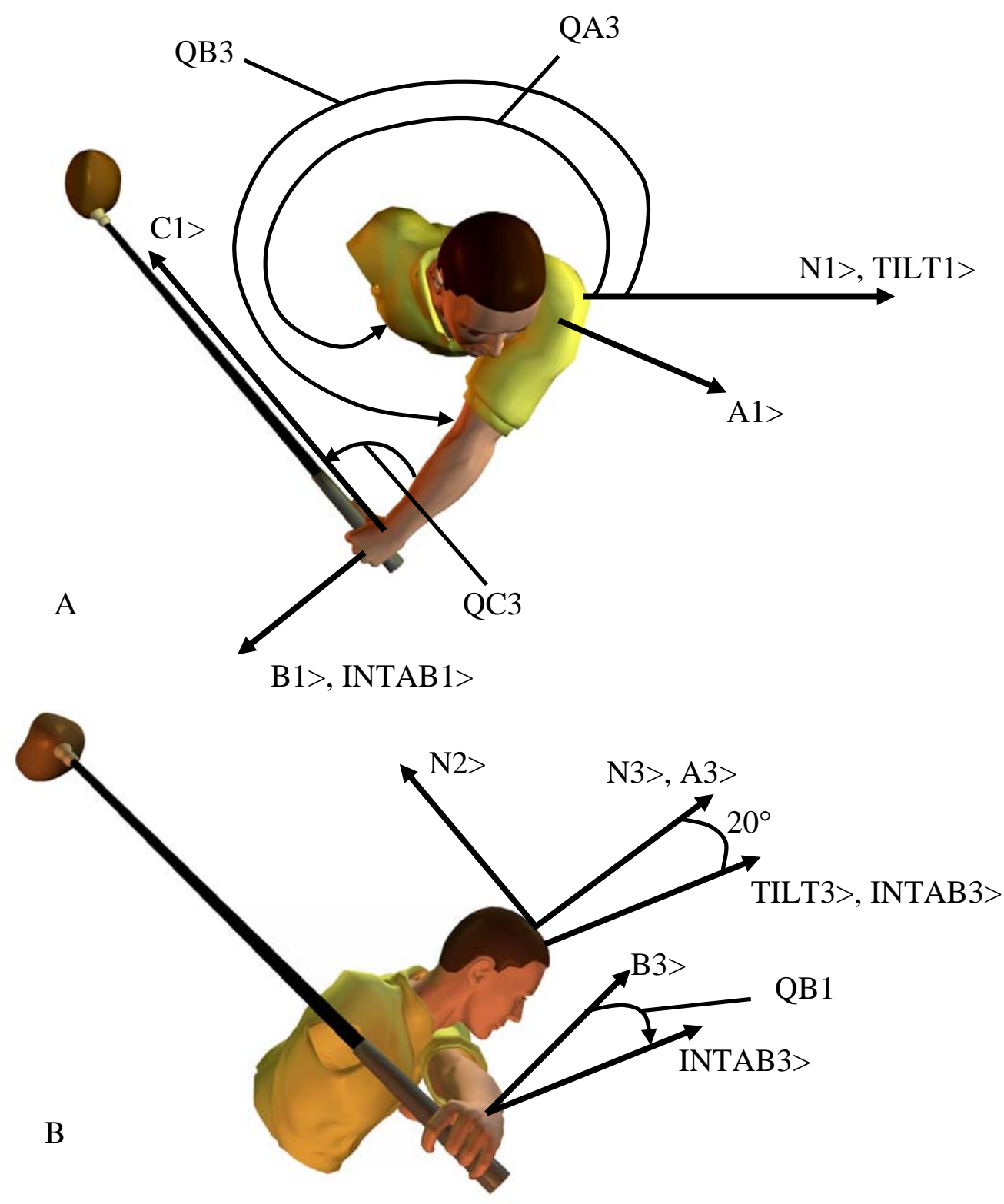

Figure 2.1.5.1 Graphical depiction of the generalized coordinates used in Autolev ${ }^{\odot}$ for developing the $3 \mathrm{D}$ golfer model. Diagram $\mathrm{A}$ is from a perspective looking along the negative TILT3> axis. Diagram B is from a perspective looking along the positive TILT1> axis.

In the Autolev ${ }^{\odot}$ code, the torso segment is referred to as Body A. Body A was constrained to rotate about the third axis (N3>) of a Newtonian reference frame fixed in 
the Autolev ${ }^{\odot}$ environment. The angular position of the torso was defined by the angle QA3. In order to have the arm segment move in a plane that was $20^{\circ}$ steeper than the torso plane, two reference frames (TILT, INTAB) were introduced. The TILT frame was rotated $20^{\circ}$ about N1> as shown in Figure 2.1.5.1B and was constrained to maintain this orientation relative to $\mathrm{N}$ during all simulations. The INTAB reference frame was constrained to rotate about the TILT3> axis. The arm segment, Body B, was attached to INTAB, and therefore also rotated about TILT3>. The angular position of the arm, in terms of horizontal abduction, was defined by the angle QB3 as shown in Figure 2.1.5.1A. To allow the arm segment to rotate about its longitudinal axis, Body B was permitted to rotate about INTAB1>. The angular position of the arm, in terms of external rotation about its longitudinal axis, was defined by the angle QB1 as shown in Figure 2.1.5.1B. The most proximal segment of the club (which includes the hand), Body C, was constrained to rotate about the third axis of the arm segment (B3>). The angular position of the most proximal club segment was defined by the angle QC3 as shown in Figure 2.1.5.1A. The reader is referred to Appendix $\mathrm{C}$ for the equations relating the velocities of the various points on the model to the generalized coordinates.

\subsection{Optimization of the Golfer-Club Model}

Two separate sets of optimization schemes were conducted to fully explore the concept of determining a precise shaft stiffness for a particular swing. The first scheme involved manipulating the swing of the model to suit a particular level of shaft stiffness. The second scheme manipulated the stiffness of the shaft to suit a particular model's 
swing. For both schemes, the objective in optimizing the golfer-club model was to maximize horizontal clubhead speed at impact with the golf ball.

\subsubsection{Customizing the Golfer to Fit the Club}

The objective in customizing the golfer model to fit the club was to maximize horizontal clubhead speed at impact with the golf ball. The objective function was equal to horizontal clubhead speed at impact minus any penalty variables accumulated during the simulated golf swing. Penalties were incurred if the model performed movements that were not executable by a human golfer, such as having the arm segment pass through the torso segment. Penalties were also incurred if the model was not in a proper position at impact, such as having the clubface misaligned with the target. The optimization scheme employed a single activation muscle control strategy where the onset of voluntary torque at each joint was controlled separately. The control variables were the onset and duration times for the four torque generators. This resulted in a total of eight control variables that were optimized to determine maximum horizontal clubhead speed at impact.

The optimization search engine was developed by myself and employed an evolutionary algorithm approach as generally expressed by Michalewicz (1996). The method was based on Darwin's theories of evolution and natural selection in which the fit individuals survive and pass on their genetic code to the next generation. In the case of the golfer-club model, fitness was measured using the objective function (clubhead speed minus penalties) and an individual was represented by a set of specific values for

the eight control variables. At the start of the optimization procedure, a population of 
individuals was randomly generated and their fitness was evaluated. The higher an individual's fitness relative to the population, the greater the chance that individual had in passing their genetic code (control variables) on to the next generation. All offspring underwent a mutation in which one or more of their control variables were modified by a random amount within a certain range of values. The range of values decreased as the generation number increased so as to facilitate narrowing in on a maximum. In addition to passing on their genetic code for mutation, the individual with the greatest relative fitness persisted unaltered into the next generation. The programming language Matlab $^{\odot}$ was used to create the optimization algorithm (Appendix D), which communicated with the golfer-club model, developed in FORTRAN ${ }^{\odot}$ (Appendix E). To evaluate each individual, values for the eight control variables were sent to the golferclub model. The objective function was evaluated in FORTRAN ${ }^{\odot}$ (a simulated swing was executed) and the value (clubhead speed minus penalties) was returned to the optimization algorithm where it represented an individual's fitness in the population. The optimization process maintained a population of 200 individuals that continually evolved over 2000 generations. The fittest individual after the $2000^{\text {th }}$ generation represented the optimal set of control variables (muscle start and stop times) for the particular model that was being optimized. Pilot work confirmed that population and generation values of 200 and 2000 consistently returned the same set of control variables. This process was repeated for each of the three golfer-club models incorporating each level of shaft stiffness. It was previously stated that three models were developed, each with a different ability to generate torque. This resulted in 9 
optimized simulations. Essentially, the process resulted in 9 separate golfer models that were each perfectly fitted to one of three clubs.

\subsubsection{Customizing the Club to Fit the Golfer}

In the previous section, methods were used to fine tune the swing of a golfer to suit a particular shaft. The purpose of this section was to fine tune shaft stiffness to suit a particular swing. It was possible that none of the three simulated clubs used in the previous section were particularly suited to any of the three golfer models. A larger and more complete spectrum of shaft stiffness was investigated in order to obtain a clearer picture of the role that shaft stiffness plays in generating clubhead speed during the golf swing.

The objective in customizing the stiffness of the shaft to the golfer model was also to maximize horizontal clubhead speed at impact with the golf ball. A single control variable, that regulated shaft stiffness, was used in the optimization scheme. The control variable represented a percentage of the original stiffness of the rotational spring-damper elements for Club-Regular. For example, Club-Regular had stiffness coefficients of 536, 159, and $87 \mathrm{Nm} / \mathrm{rad}$. Therefore, at the 50\% stiffness level, the stiffness coefficients were $268,79.5$, and $43.5 \mathrm{Nm} / \mathrm{rad}$. An exhaustive search was performed by evaluating all stiffness levels from 50 to $150 \%$ in $1 \%$ increments. This range of stiffness was chosen to ensure that it encompassed the maximum range of stiffness values that could be practically developed by manufacturers. For every evaluation in the exhaustive search, the muscle control strategy used was that found for 
the optimization of Golfer-Medium with Club-Regular in the previous section. This procedure was also completed for Golfer-Slow and Golfer-Fast with Club-Regular.

\subsection{Simulated Thought Experiments using the Golfer-Club Model}

One of the big advantages of a mathematical model is that it permits experimentation that would not be practical or in some cases not even possible in the real world. Although it may have no direct application, information gained from thought experiments can certainly help develop a deeper understanding of a phenomenon. It is for this reason that additional simulations with the golfer-club model were conducted.

\subsubsection{Use of a Perfectly Rigid Shaft}

It is not known to what extent a shaft can store and release energy in a useful way during the golf swing. Even though it is not possible with live golfers, a comparison of clubhead speeds attained with a flexible shaft versus a perfectly rigid shaft would help answer the question. Once again, the three golfer models that only differed in their ability to generate swing speed were employed. Each of the three golfer models had the timing of their control torques optimized using the same method detailed in section 2.2.1. However, for these optimizations, the golfer models were matched with a perfectly rigid shaft. This shaft will be referred to as Club-Rigid. The shaft was modelled as a single rigid link by incorporating motion constraints at the three joints that connected the four club segments. The equations of motion used in the model were reformulated in order to represent these constraints. Clubhead speeds attained by the 
three rigid shaft models were compared to the clubhead speeds generated by their respective non-rigid shaft models.

\subsubsection{Repositioning the Clubhead Center of Mass}

Several authors have stated that the shaft is deflected in the lead direction at impact due to the radial force acting on the center of mass of the clubhead which is offset from the axis of the shaft (Horwood, 1994; Jorgensen, 1994; Milne \& Davis, 1992). The effect of the radial force will be to pull the center of mass of the clubhead into a position that is collinear with the line of action of the radial force. If the radial force is the dominating factor controlling shaft deflection at impact, then reversing the position of the clubhead's center of mass should reverse the direction of shaft bending. If the center of mass is geometrically moved into a position in front of (positive $y$ direction) the longitudinal axis of the shaft (Fig. 1.2.1.1), then theoretically, the shaft should be deflected in the lag direction at impact. This would be analogous to a lefthanded golfer swinging a right-handed driver. Similarly, if the center of mass of the clubhead is positioned in-line with the shaft axis, then no deflection in the lead/lag directions should be evident at impact with the ball.

The same theory holds for shaft deflection in the toe-up/toe-down direction. It has been suggested that, at impact, the shaft is deflected in the toe-down direction due to the action of radial force acting on the off-set center of mass of the clubhead (Horwood, 1994; Jorgensen, 1994; Milne \& Davis, 1992). According to this theory, if the center of mass is moved into a position below (negative $x$ direction) the longitudinal axis of the shaft (Fig. 1.2.1.1), then the shaft would be deflected in the toe-up direction at impact. 
Likewise, if the center of mass was positioned in-line with the shaft axis, then the shaft should be in a neutral toe-up/toe-down position at impact.

The above scenarios were tested using the optimized swing of Golfer-Medium with Club-Regular. For each condition, the position of the center of mass of Club_seg_4 (the most distal club segment) was only changed along either the $x$ axis or the $y$ axis, and not both simultaneously. Shaft deflections were compared for three positions of the center of mass along each axis: normal, in-line, and reversed. Normal refers to the position of the center of mass in a typical driver. In-line refers to the center of mass being collinear with the longitudinal axis of the shaft. Reversed refers to the placement of the center of mass in the exact opposite location along a particular axis. For example, normally the center of mass of Club_seg_ 4 is located $4.7 \mathrm{~cm}$ in the negative $y$ direction (Fig. 1.2.1.1 and Table 2.1.1.1). Therefore the reversed condition would be to place the center of mass at $4.7 \mathrm{~cm}$ in the positive $y$ direction. These tests were conducted to gain a better understanding of the role of radial force in affecting shaft deflection during the swing, and altering clubhead orientation at impact.

\subsubsection{Removal and Isolation of Radial Force}

Changing the position of the clubhead's center of mass does help foster an understanding of the influence of radial force on shaft bending. However, even if the center of mass is geometrically placed in-line with the shaft, the shaft can still bend due to tangential forces resulting in the clubhead center of mass no longer being collinear and resulting in radial force once again exerting its influence. 
Earlier, in section 1.2.5, I reasoned that resolving the forces applied to the club by the golfer into tangential and radial components would help garner a better understanding of shaft bending. I then described simple experiments that would demonstrate the effects of these forces when acting in isolation. However, during the golf swing, these forces never act in isolation and it would be a very difficult task to tease out the specific effects of each force at any point in the swing. To gain a further comprehension of the influence of radial force, shaft deflection could be compared between a club swung in a normal fashion and a club swung in the absence of radial force. In reality, it is not possible to swing a real golf club without applying a radial force at the grip end, but it is possible to conduct such an experiment with a mathematical model.

A simulation was conducted with the optimized Golfer-Medium।Club-Regular combination. The force and torque values applied to the club during the swing were recorded at each time step. The force and torque vectors were each broken down into three components based on a reference frame fixed in the grip end of the club (Fig. 1.2.1.1). A second simulation was conducted with just the four segment Club-Regular model in which the six force and torque measures taken at each time step in the previous simulation served as input. As would be expected, the resulting clubhead speed and shaft deflection measurements were identical to the first simulation in which GolferMedium swung Club-Regular. A third simulation was performed in which the values for the radial force at each time step were set to $0 \mathrm{~N}$, but the other force and torque measures remained the same. This allowed the effect of radial force to be removed from the golf swing. A fourth simulation was performed in which only the values for radial 
force were input at each time step, and all other force and torque measures were set equal to zero. This allowed the effect of radial force to be isolated during the downswing.

\subsubsection{Effect of Shaft Deflection on Clubhead Orientation}

Since the clubhead is rigidly fixed to the shaft, any amount of shaft deflection will result in a change in clubhead orientation at impact and, therefore, ball flight trajectory. With respect to the successful execution of a golf shot, shaft deflection can alter the initial trajectory of the golf ball relative to two important planes. The first plane is vertical and runs along the target line. Assuming a right-handed golfer, if shaft deflection resulted in a closed clubface, then the initial ball trajectory will be to the left of this vertical plane. If shaft deflection resulted in an open clubface, then the initial ball trajectory will be to the right of the vertical plane. The second important plane is simply represented by the horizontal surface of the ground. Relative to this plane, shaft deflection can either result in a higher or lower ball flight trajectory. Of course, closing the clubface, or adding more loft can also result from the position in which the golfer orients the club at impact. To avoid ambiguity, any change in clubhead orientation at impact relative to the two aforementioned planes that resulted entirely from shaft deflection will be referred to as dynamic. For example, if shaft deflection results in an additional two degrees of loft, then that two degrees will be referred to as dynamic loft. Likewise, if shaft deflection results in the clubface being directed two degrees to the left of the target line, then that two degrees will be referred to as dynamic close. 
Shaft deflection about either the $x$ or $y$ axis (Fig. 1.2.1.1) alone will have an effect on both dynamic loft and dynamic close. In other words, both dynamic loft and dynamic close are a function of two variables, namely, lead deflection and toe-down deflection. In order to quantify the effects that shaft deflection has on clubhead orientation, two 3D plots were generated. One plotted dynamic loft versus toe-down and lead deflections, while the second plotted dynamic close versus toe-down and lead deflections. The range of lead and toe-down deflections used for graphing dynamic loft and dynamic close were based on the maximum shaft deflection values attained during the optimized swing of Golfer-Medium with Club-Regular.

\subsection{Model Validation}

\subsubsection{External Validity}

\subsubsection{Live Golfer Testing}

Four golfers with handicaps ranging from 3 to 30 were recruited to have their swings filmed using high-speed video. Each golfer used three separate drivers; three times each, from two perspectives for a total of 18 swings per golfer. From Perspective 1, participants were filmed with the camera perpendicular to the plane of the golf swing. In order to orient the camera in a position that was approximately perpendicular to the swing plane, the camera was placed $5.1 \mathrm{~m}$ away horizontally, and $5.5 \mathrm{~m}$ above ground level (Fig. 2.4.1.1.1). From Perspective 2, the camera was positioned $4 \mathrm{~m}$ behind the golfer pointing in the intended ball flight direction, such that at impact, the longitudinal axis of the shaft would be perpendicular to the camera (Fig. 2.4.1.1.2). The three drivers used during testing differed only in the flexibility of their shafts. These were the same 
drivers used to determine the stiffness parameters for the simulated golf clubs. One shaft was flexible (Club-Flexible), a second shaft had regular stiffness (Club-Regular), and the third shaft was stiff (Club-Stiff). Each shaft was $110.5 \mathrm{~cm}$ long from the top of the grip to its insertion point into the clubhead, and each club had a total mass of $310 \mathrm{~g}$. The participants were blinded to shaft flexibility and the order in which each driver was swung was randomized. Blinding was accomplished by using clubs that had no markings that would allow the participants to distinguish shaft stiffness. Further, participants were not permitted to "waggle" or test the stiffness of the shaft at any point during data collection. 


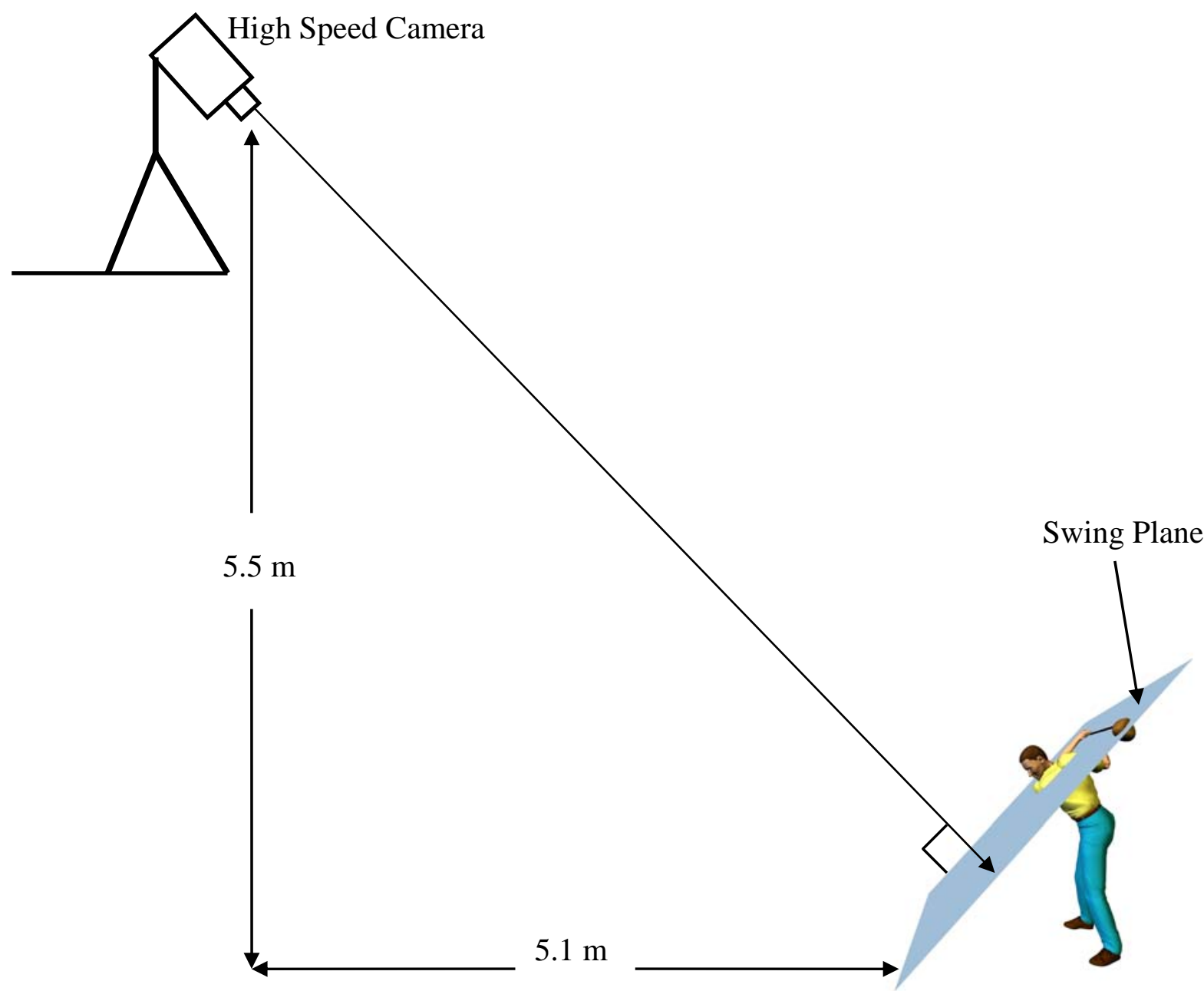

Figure 2.4.1.1.1 Set-up of live golfer testing from Perspective 1. 


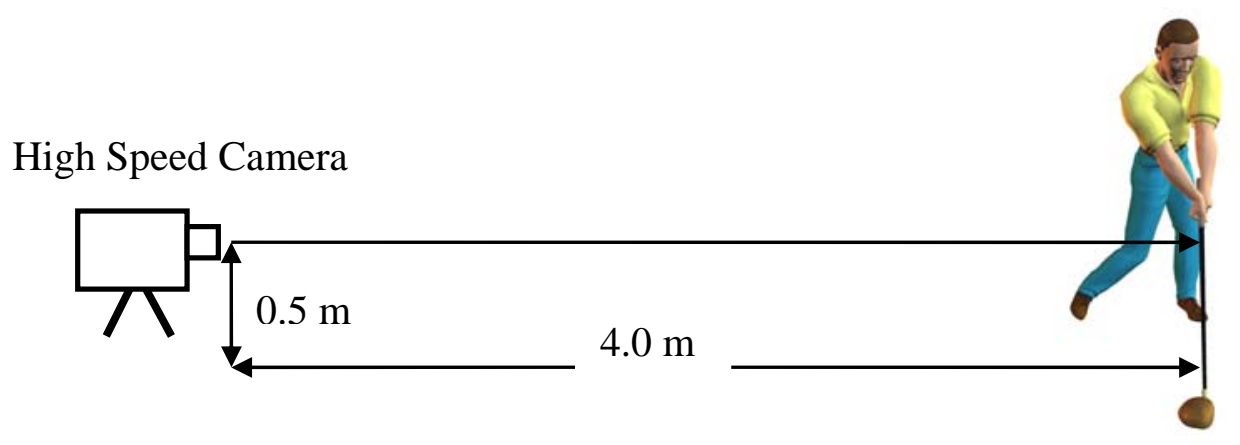

Figure 2.4.1.1.2 Set-up of live golfer testing from Perspective 2.

Each participant completed a warm-up consisting of ten swings with a fourth driver not used in the study. Participants were able to hit a golf ball into a net for all practice and actual swings. Participants were instructed to swing consistently from trial to trial and to swing as they normally would on the golf course.

Four thin strips of reflective tape were wrapped around the shaft at equal intervals along its length. The first strip was placed immediately after the grip with the final strip being placed at the insertion of the shaft into the clubhead. This enabled the shaft to be modeled as three segments. All swings were captured using a high-speed digital video camera (MotionScope PCI 1000). From Perspective 1, the sampling rate was 500 fps with a shutter speed of $1 / 1500$ s. In order to maintain a high level of resolution, the camera had a built in function that reduced the field of view as sampling rate increased. Since the field of view shrunk with increases in sampling rate, $500 \mathrm{fps}$ 
was the maximum sampling rate that could be used without excluding sections of the golf swing. Also, due to size constraints of the testing lab, it was not possible to move the camera further away from the participants, so as to increase the field of view. From Perspective 2, the sampling rate was $1000 \mathrm{fps}$ with a shutter speed of $1 / 3000 \mathrm{~s}$. Only three of the four golfers participated in the filming from Perspective 2. Also, a fifth golfer (left-handed), videoed from Perspective 1, completed three swings with ClubRegular while swinging left-handed.

Each swing was analyzed using the motion analysis software HU-M-AN ${ }^{\odot}$. Displacement data throughout the swing for each strip of reflective tape was generated by manually digitizing the center of each strip in each frame. The raw coordinate data were smoothed using HU-M-AN's built-in $4^{\text {th }}$ order recursive Butterworth filter. Cutoff frequencies, ranging from 15 to $19 \mathrm{~Hz}$, were individually selected for each of the $\mathrm{X}$ and Y coordinate data sets for each marker based on their residual plots. The smoothed displacement data were numerically differentiated to determine velocity data for each marked point on the shaft.

Shaft deflection for the live golfer testing was calculated in the same manner described in section 1.2.1 and demonstrated in Figure 1.2.1.2. However, there were two differences. First, from Perspective 1, shaft bending was only measured within the plane of the swing and without any distinction between lead/lag and toe-up/toe-down deflections. However, for the first three fifths of the downswing, the in-plane shaft bending is almost completely in the toe-up/toe-down direction. While during the final tenth of the downswing, in-plane bending is primarily in the lead/lag direction. Due to the club rotating through $90^{\circ}$ about the longitudinal axis of the lead arm, shaft deflection 
during the later part of the downswing is some combination of lead/lag and toe-up/toedown deflection. The second difference concerns the placement of the points along the shaft used for forming the vectors in determining the magnitude and direction of shaft deflection. In the modeled golf swing, the most proximal point on the shaft was the top of the grip. Since this point was obscured during the live golfer testing, the most proximal point was at the bottom of the grip, and the second most proximal point was approximately $27 \mathrm{~cm}$ further down the shaft. This second difference assumes that there is negligible bending in the first $27 \mathrm{~cm}$ of the shaft below the grip. For this reason, the magnitude of shaft deflections measured during the live golfer testing will likely be an underestimate. Also, the most distal point was at the hozel and not at the center of mass of the clubhead, as used during the simulated swings. This would also likely result in smaller measured shaft deflections.

\subsubsection{Error and Reliability of Live Golfer Testing}

To estimate the error associated with the videoing and digitizing process, the length of the first segment of the club was calculated for every frame of every swing. A mean, standard deviation, total range and coefficient of variation were then determined based on all of the length measurements for each swing. An average of each of these statistics was then determined across each swing. These parameters were compared with the true length of the first segment.

An estimate of the reliability in determining shaft deflection was calculated using the 95\% limits of agreement method put forth by Altman and Bland (1983) and endorsed by Atkinson and Nevill (1998). Aside from the data presented in this thesis, 
each measure of shaft deflection was calculated on two other occasions. The standard deviation of the difference scores between the two occasions was multiplied by 1.96 to determine the $95 \%$ limits of agreement for a measurement of shaft deflection. Essentially this means that if the same video data were analyzed twice, to determine shaft deflection, then there would be a 95\% chance that the difference between the two deflection values would fall with-in the limits of agreement.

\subsubsection{Swing Plane Comparison}

Shaft bending aside, the ability of the model to move the club in a similar pattern to actual golfers is an important validity concern. A good measure of comparison is the swing plane. Currently, a mathematical model of golfer with varying swing plane does not exist in the literature. The popular method of modeling the downswing as a $2 \mathrm{D}$ motion was likely initially brought about by the necessity of a computationally simple system due to the technology available at the time (Cochran \& Stobbs, 1968; Jorgensen, 1970; Lampsa, 1975; Williams, 1967). Vaughn (1981), upon conducting a 3D kinematic analysis of expert golfers, determined that the plane of the downswing varies throughout the movement. This was more recently confirmed by Coleman and Rankin (2005). A swing plane generated by the mathematical model used in this thesis was compared to the swing plane measurements made by Coleman and Rankin in section 3.9. 


\subsubsection{Internal Validity}

To ensure that the 3D equations of motion were correct and behaving in accordance with Newton's Laws of Motion a simple simulation was performed. The potential and kinetic energies of the model's six segments were calculated as they rotated about a horizontal axis that passed through the middle of the torso segment. For this simulation, no constraints were placed on the motion of the segments. Essentially, the model behaved like a multi-segment pendulum solely under the influence of gravity. If the sum of the potential and kinetic energies of the model remained constant during the simulation, then it would be a strong indication that the equations of motion were generated correctly. Also, an integration checking function provided by Autolev ${ }^{\circledR}$ was implemented during each simulation to ensure that the integration was accurate to within seven significant digits. Basically, the integration checking function determined if the total force and torque inputs into the system balanced out with the resulting kinematics. 
3

RESULTS 
The format of the results section mirrors the sequence of experiments described in the methods section. For each methods section there is a corresponding results section with all pertinent findings that emerged from the experiments presented. Due to the size of this document, it is recommended that a review of the corresponding method section is made prior to reading each specific result section.

\subsection{Optimization of the Golfer-Club Model}

\subsubsection{Typical Swing Results}

The torque profiles for each of the 9 optimized simulations demonstrated the same proximal to distal pattern of coordination (Fig. 3.1.1.1). The torso muscle torque generator was always activated at $\mathrm{t}=0$, and was followed in sequence by the shoulder, wrist, and arm rotation torques. Note that arm rotation refers to the external rotation of the lead arm about its longitudinal axis during the downswing. For all simulations, the torso, shoulder, and arm rotation torques remained active for the entire simulation. The arm's external rotation torque dropped to zero before the end of the simulation (Fig. 3.1.1.1). This occurred due to the force-velocity property imposed on the muscle torque generator. As impact approached, the external rotation velocity of the arm segment was too large for the muscle torque generator to apply an external rotation moment to the segment. 


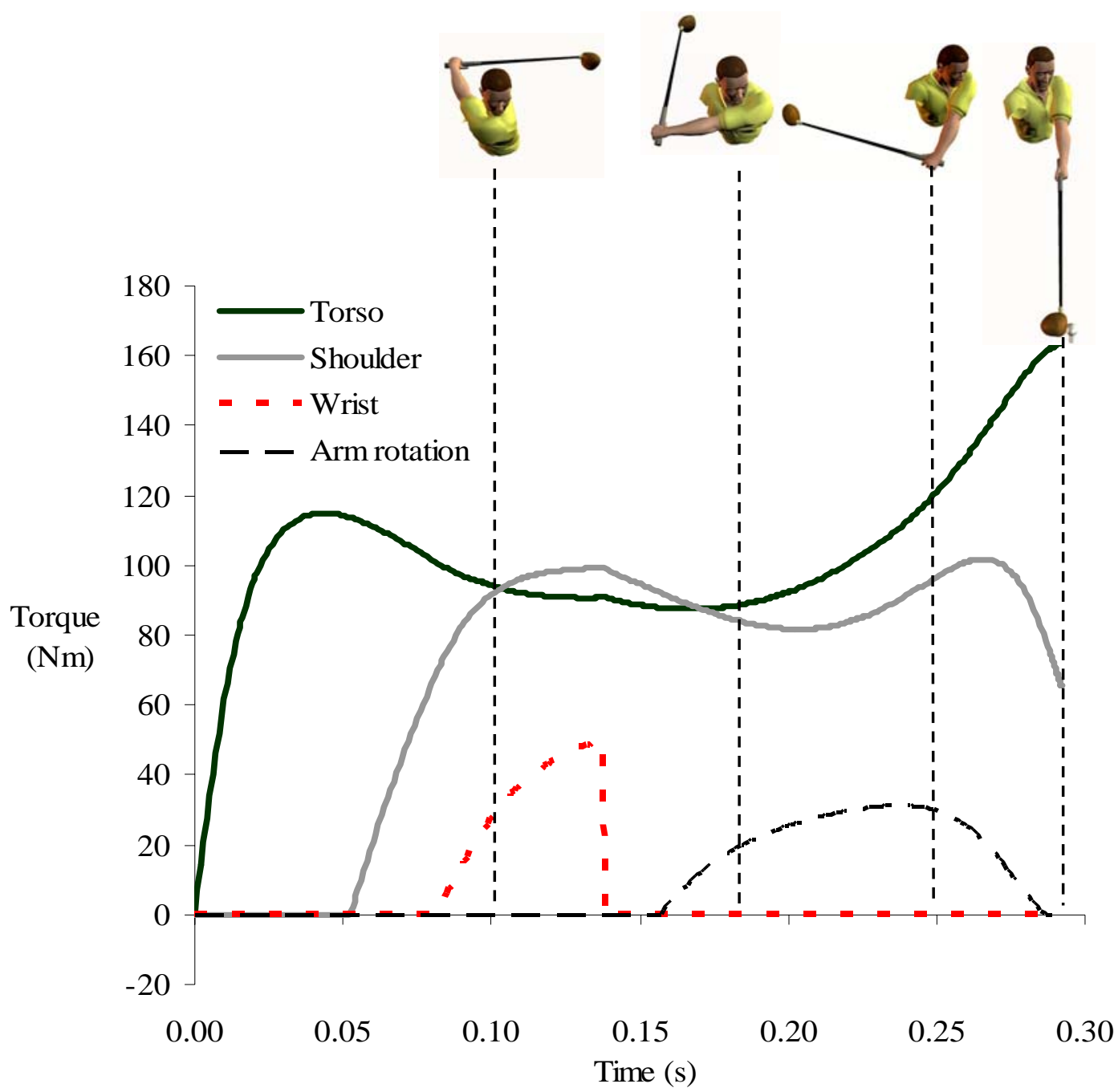

Figure 3.1.1.1 Output from the four muscle torque generators during the optimized execution of Golfer-Medium with Club-Regular.

The generalized coordinates, which define the angular positions of the segments of the golfer model, followed the same proximal to distal pattern as the torque generators (Fig. 3.1.1.2). Torso rotation, which had an initial angular position of $270^{\circ}$, showed an initial move in the negative direction. This negative rotation is supported by the fact that the torso segment was given initial angular velocity in the backswing direction. The torso began rotating into the downswing at approximately $0.05 \mathrm{~s}$. Shoulder rotation, or the angular position of the arm in the horizontal abduction plane, is 
depicted in terms of its relative angular position to the torso segment (Fig. 3.1.1.2), instead of its absolute angular position as shown in Figure 2.1.5.1. Shoulder rotation, relative to the torso segment, was initiated approximately 0.10 seconds into the simulation. The angular position of the hand relative to the arm segment begins to change next at approximately 0.12 seconds into the simulation. The final relative movement to be initiated is the rotation of the lead arm about its longitudinal axis which is responsible for squaring the clubface at impact. This rotation doesn't occur until approximately 0.20 seconds into the downswing. 


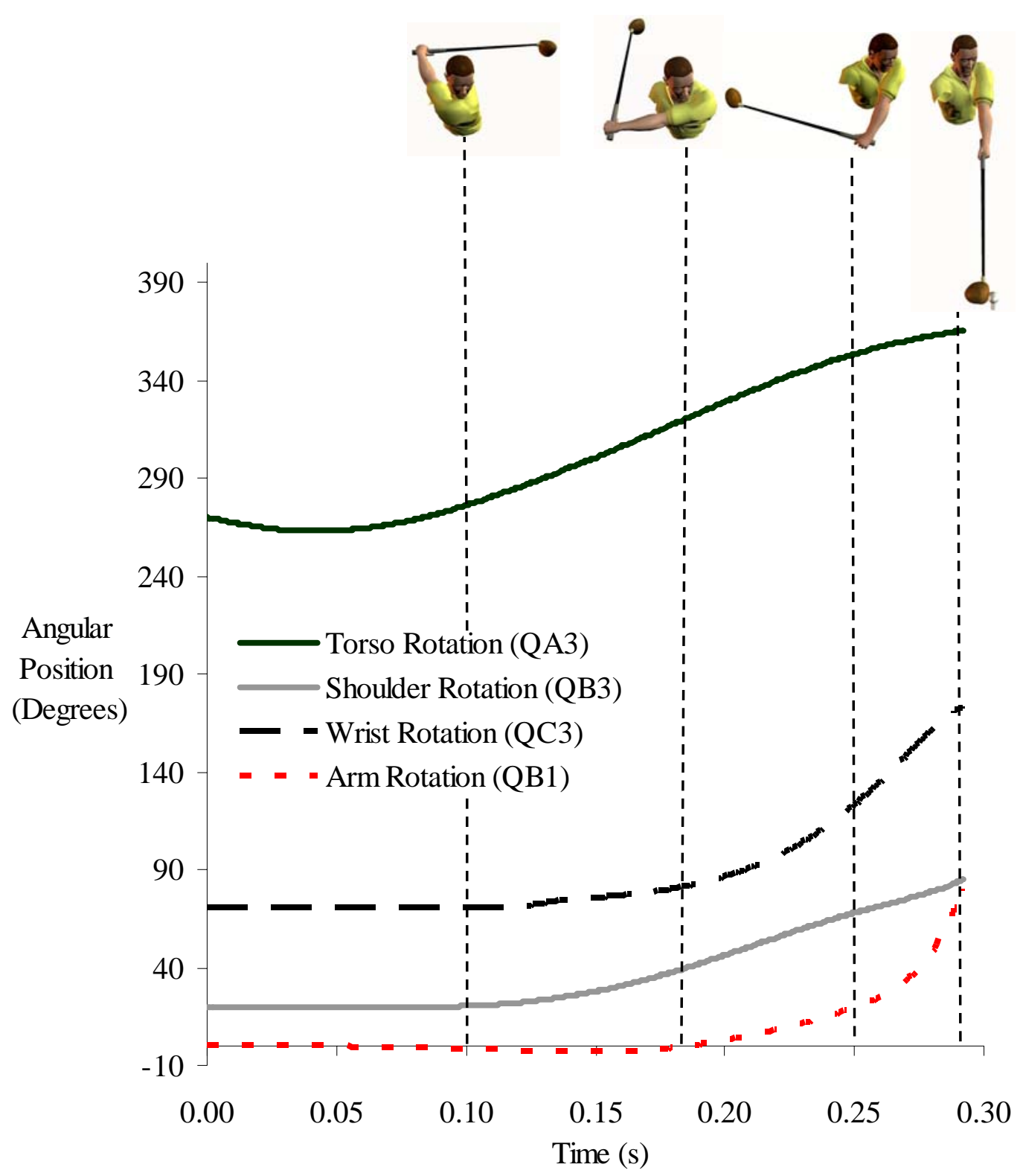

Figure 3.1.1.2 Generalized coordinates of the golfer-model as a function of time. Shoulder rotation is provided relative to the angular position of the trunk and not in absolute terms as described in section 2.1.5 and in Appendix C.

\subsubsection{Customizing the Golfer to Fit the Club}

Regardless of shaft stiffness, if the same torque generator parameters were used, the clubhead speeds attained by the golfer models were similar (Table 3.1.2.1). The largest difference in clubhead speed $(0.08 \mathrm{~m} / \mathrm{s})$ for a particular level of swing speed 
occurred with the Golfer-Medium model between Club-Stiff (45.04 m/s) and ClubFlexible (44.96 m/s).

The constraints imposed on the optimization routine by the penalty variables resulted in some imprecise relationships for the quantities presented in Table 3.1.2.1. For example, a clear relationship did not exist for the 'max toe-up’ measurement across levels of shaft stiffness for the Golfer-Medium model (Table 3.1.2.1). The optimized muscle coordination strategy that was determined for the Golfer-Medium/Club-Flexible arrangement would have resulted in poor clubface alignment at impact for the GolferMedium/Club-Regular pairing. Therefore, the optimization algorithm determined a different optimal coordination strategy for that particular pairing which deflected the shaft differently, but still resulted in a similar clubhead speed at impact. If identical swings were used for each shaft, then specific mathematical relationships would have become apparent, but this would have come at the expense of an optimized swing with a correct impact position. This idea is explained more fully in the next section. The toedown deflections at impact also failed to demonstrate a clear relationship across conditions. This was also related to the slight variations in muscle coordination strategies between conditions.

Even with the confounding nature of the penalty variables, some clear relationships amongst the shaft deflection measurements emerged across swing speed and shaft stiffness conditions.

For every optimized swing, the maximum lead deflection of the shaft occurred at impact. It was also apparent that lead deflection increased as shaft stiffness decreased and that lead deflection decreased as swing speed decreased. For example, the largest 
lead deflection occurred with the Golfer-FastlClub-Flexible pairing $(7.20 \mathrm{~cm})$, while the smallest lead deflection was measured when Golfer-Slow was matched with Club-Stiff $(5.74 \mathrm{~cm})$. The same pattern materialized with the 'max lag' data. The largest lag measurement was recorded during the Golfer-FastlClub-Flexible simulation $(-4.79 \mathrm{~cm})$, while the smallest lag value was found during the Golfer-SlowiClub-Stiff simulation ($2.70 \mathrm{~cm})$.

Table 3.1.2.1 Measures of shaft bending during the swing and at impact for 9 optimized golfer-club models.

\begin{tabular}{|c|c|c|c|c|c|c|c|c|c|}
\hline Swing Speed Level & \multicolumn{3}{|c|}{ Golfer-Slow } & \multicolumn{3}{|c|}{ Golfer-Medium } & \multicolumn{3}{|c|}{ Golfer-Fast } \\
\hline Shaft Stiffness Level & $\begin{array}{l}\text { Club } \\
\text { Flex. }\end{array}$ & $\begin{array}{l}\text { Club } \\
\text { Reg. } \\
\end{array}$ & $\begin{array}{l}\text { Club } \\
\text { Stiff } \\
\end{array}$ & \begin{tabular}{|l|} 
Club \\
Flex.
\end{tabular} & \begin{tabular}{|l|} 
Club \\
Reg. \\
\end{tabular} & $\begin{array}{l}\text { Club } \\
\text { Stiff } \\
\end{array}$ & $\begin{array}{l}\text { Club } \\
\text { Flex. }\end{array}$ & \begin{tabular}{|l|} 
Club \\
Reg. \\
\end{tabular} & $\begin{array}{l}\text { Club } \\
\text { Stiff } \\
\end{array}$ \\
\hline Clubhead Speed (m/s) & 38.02 & 38.05 & 37.98 & 44.96 & 44.98 & 45.04 & 52.94 & 52.96 & 52.94 \\
\hline Kick Velocity (m/s) & 5.04 & 4.84 & 4.89 & 7.14 & 6.95 & 6.88 & 10.51 & 9.65 & 9.55 \\
\hline Max Kick Velocity (m/s) & 5.07 & 4.88 & 4.91 & 7.19 & 6.98 & 6.89 & 10.52 & 9.68 & 9.56 \\
\hline Lead (cm) & 5.83 & 5.78 & 5.74 & 6.57 & 6.25 & 6.03 & 7.20 & 6.87 & 6.66 \\
\hline Max Lead (cm) & 5.83 & 5.78 & 5.74 & 6.57 & 6.25 & 6.03 & 7.20 & 6.87 & 6.66 \\
\hline Toe-down (cm) & -1.98 & -1.95 & -1.79 & -2.25 & -2.26 & -2.11 & -2.49 & -2.54 & -2.42 \\
\hline Max Toe-down (cm) & -1.98 & -1.96 & -1.80 & -2.25 & -2.27 & -2.12 & -2.50 & -2.55 & -2.45 \\
\hline Dynamic Loft (deg) & 4.68 & 4.62 & 4.42 & 5.47 & 5.20 & 4.82 & 6.27 & 5.97 & 5.54 \\
\hline Dynamic Close (deg) & 4.19 & 4.15 & 4.01 & 4.75 & 4.49 & 4.23 & 5.17 & 4.97 & 4.65 \\
\hline Max Lag (cm) & -3.00 & -2.82 & -2.70 & -3.67 & -3.62 & -3.43 & -4.79 & -4.40 & -4.28 \\
\hline Max Toe-up (cm) & 8.37 & 8.60 & 7.19 & 8.72 & 10.2 & 9.59 & 10.69 & 10.09 & 9.74 \\
\hline Time to Max Toe-up (s) & .159 & .145 & .146 & .096 & .137 & .129 & .096 & .094 & .133 \\
\hline
\end{tabular}

The dynamic loft (Fig. 3.1.2.1) and dynamic close data (Fig. 3.1.2.2) revealed the same pattern across conditions as the lead deflection data. This finding was not surprising since dynamic loft and dynamic close are completely dependent upon the 
amount of toe-down and lead deflection present at impact. The relationship between these variables is described more fully in section 3.6. The Golfer-FastlClub-Flexible model produced the largest dynamic loft $\left(6.27^{\circ}\right)$ and dynamic close $\left(5.17^{\circ}\right)$, while Golfer-Slow $\backslash$ Club-Stiff generated the smallest dynamic loft $\left(4.42^{\circ}\right)$ and dynamic close $\left(4.01^{\circ}\right)$. It is important to note that these values of dynamic close were generated without any ability of the shaft to twist about its longitudinal axis.

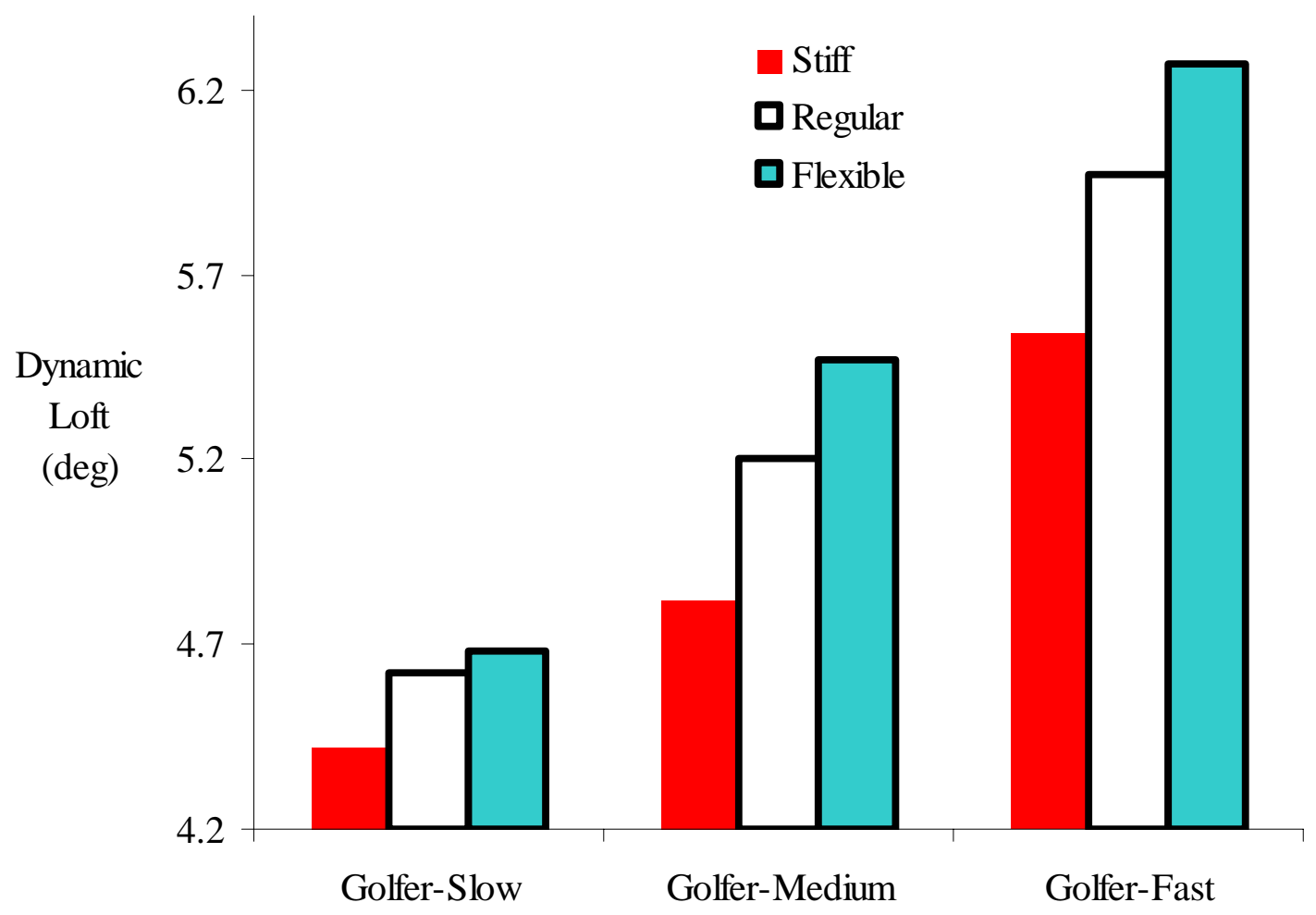

Figure 3.1.2.1 Dynamic loft at impact from the 9 optimized simulations in which the timing of each golfer model's swing was fit to each of the clubs. 


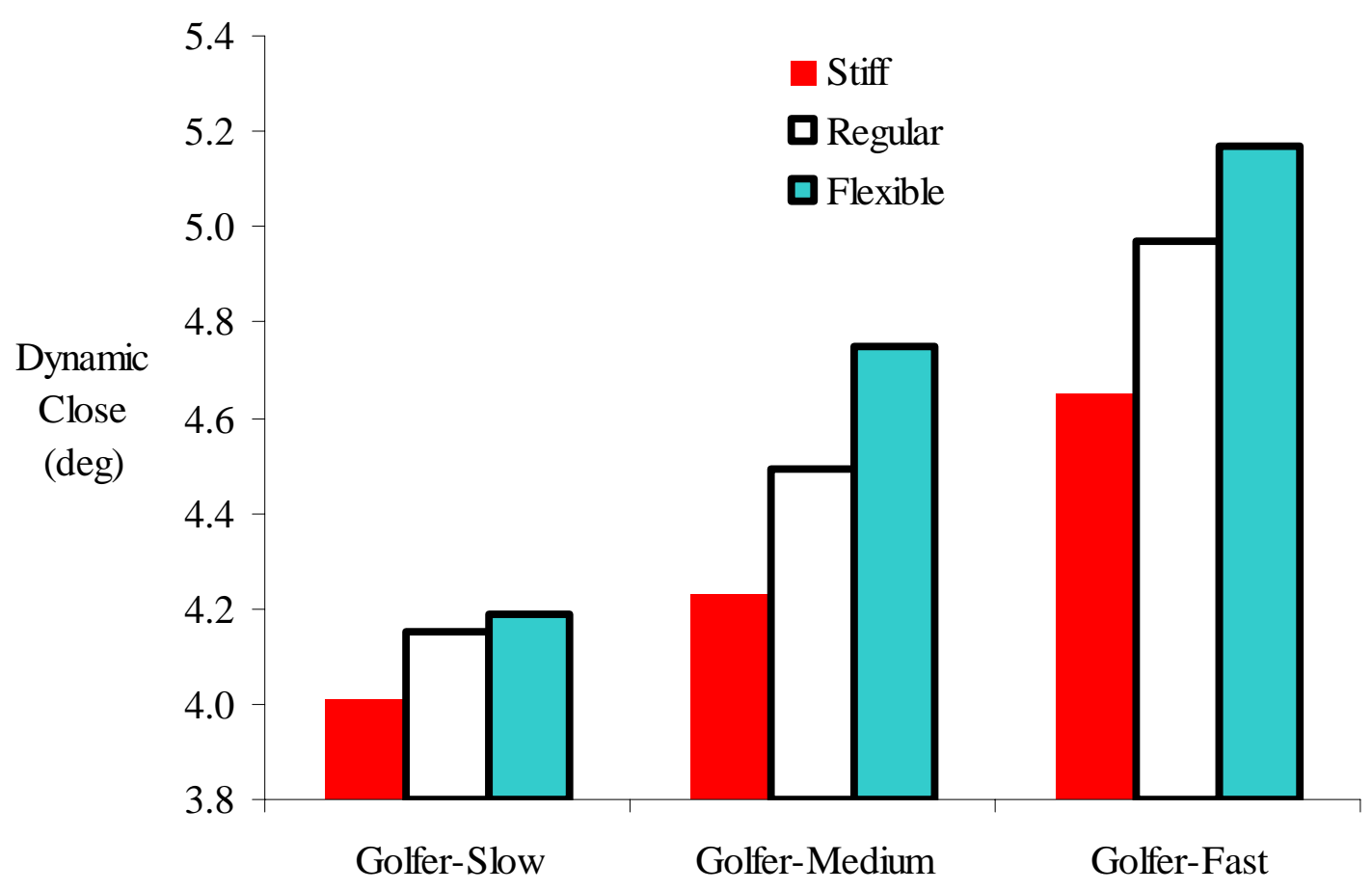

Figure 3.1.2.2 Dynamic close at impact from the 9 optimized simulations in which the timing of each golfer model's swing was fit to each of the clubs.

Shaft deflection curves in both the lead/lag and toe-up/toe-down directions showed similar shapes in all simulations (Fig. 3.1.2.3). In all simulations, maximum lead deflection occurred at impact and maximum toe-down deflection occurred very near impact. For the final third of the downswing, the club gradually bends into its maximum toe-down position. While over the final five hundredths of a second, the club quickly moves from its maximum lagging position into its maximum leading position at impact signifying a large kick velocity close to impact. The shaded area of the graph indicates the portion of the downswing in which the club was in a lagging position prior to impact. In this lag position, strain energy was stored in the shaft and therefore had 
the potential to be released and result in the kick velocity that would deflect the shaft into a leading position at impact.

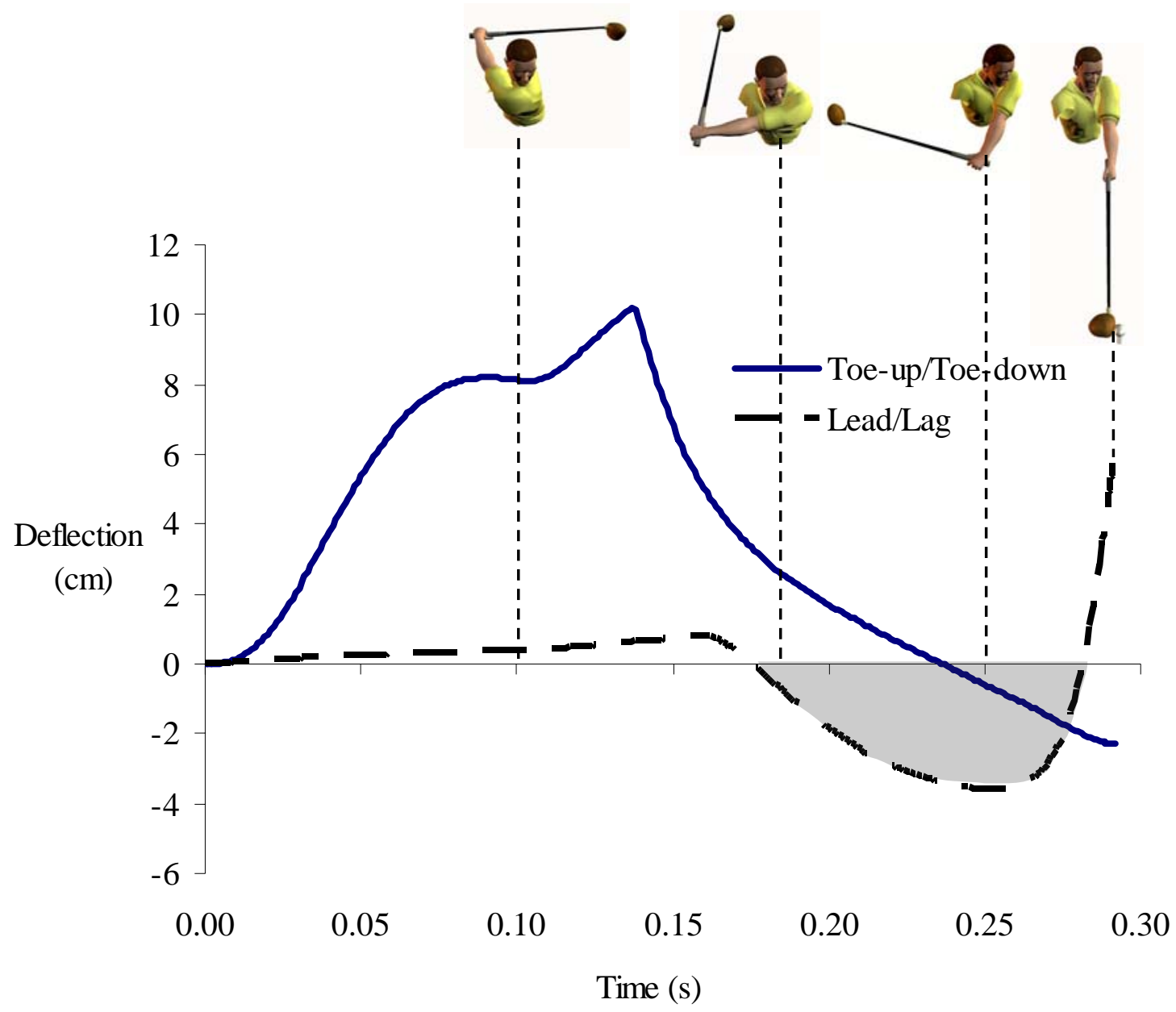

Figure 3.1.2.3 Toe-up/toe-down and lead/lag deflections during the optimized execution of Golfer-Medium with Club-Regular. The shaded area represents the time during which the clubhead was deflected in the lag direction.

Mathematically, kick velocity is defined as the derivative of lead/lag deflection with respect to time. Conceptually, it can be loosely thought of as the shaft's contribution to clubhead speed for a particular swing. For all optimized simulations, kick velocity peaked nearly simultaneously with impact (Fig. 3.1.2.4, Table 3.1.2.1). 
This suggests that kick velocity played an import role in the overall maximization of clubhead speed. For example, the Golfer-Medium\Club-Regular simulation demonstrated that kick velocity contributed approximately $7 \mathrm{~m} / \mathrm{s}$ more clubhead speed than if a perfectly rigid club was swung with the same golfer kinematics (Fig. 3.1.2.4).

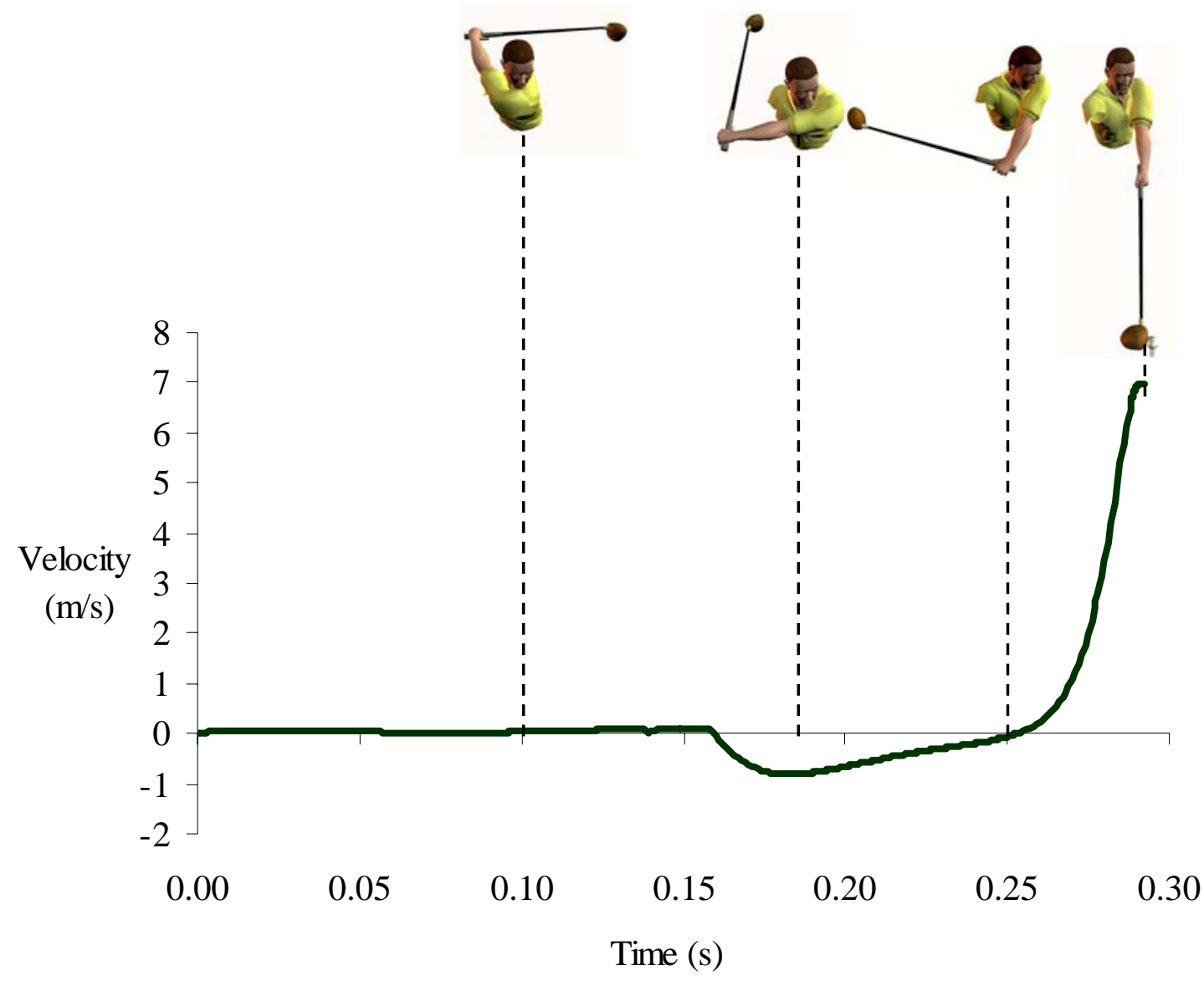

Figure 3.1.2.4 Kick velocity during the optimized execution of Golfer-Medium with Club-Regular. 


\subsubsection{Customizing the Club to Fit the Golfer}

Evaluating a complete range of shaft stiffness with a single swing pattern from Golfer-Medium revealed that clubhead speed increased as shaft stiffness decreased (Fig.

3.1.3.1). The highest horizontal clubhead speed at impact $(46.10 \mathrm{~m} / \mathrm{s})$ was attained at the most flexible shaft stiffness level of 50\%. This clubhead speed was approximately $1.1 \mathrm{~m} / \mathrm{s}$ faster than the clubhead speed attained when Golfer-Medium was optimized to Club-Regular $(44.98 \mathrm{~m} / \mathrm{s})$. However, the shaft stiffness level of 50\% also resulted in the largest accumulation of penalties. The increased clubhead speeds associated with more flexible shafts were also accompanied by improper clubhead orientations and positions at impact as demonstrated by the plot of 'clubhead speed - penalties’ (Fig. 3.1.3.1). The value of the objective function (clubhead speed - penalties) demonstrated that the best golf swing occurred at a stiffness level of 100\% (Fig. 3.1.3.1). This finding supports the results of the optimization from the previous section. The optimal shaft stiffness for Golfer-Medium, was the same shaft stiffness it was originally optimized with, namely, Club-Regular. 


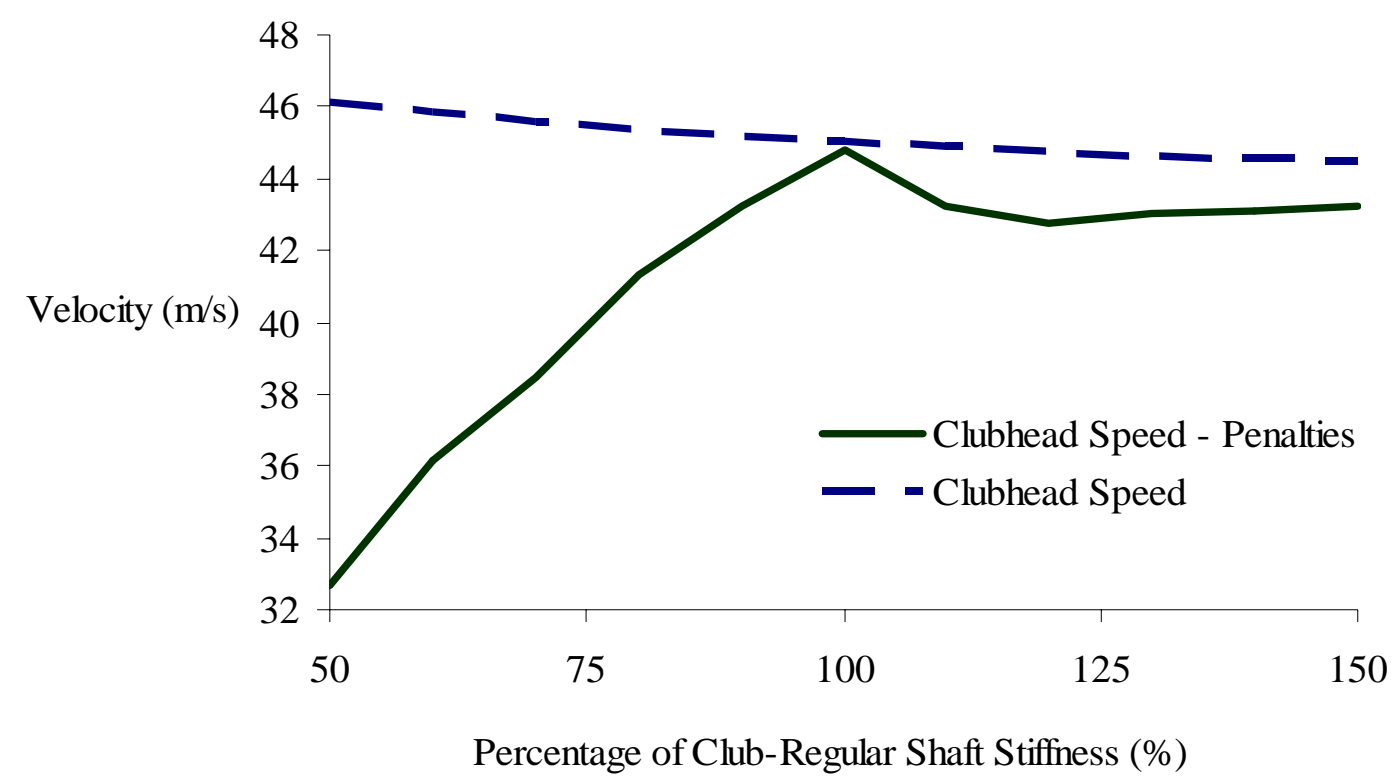

Figure 3.1.3.1 Resulting clubhead speed and clubhead speed minus penalties over a complete range of shaft stiffness for a single swing pattern from Golfer-Medium.

Similar results were found with Golfer-Slow and Golfer-Fast (Fig. 3.1.3.2 and Fig. 3.1.3.3). Clubhead speed increased as shaft stiffness decreased, but at the expense of proper impact conditions. The best swings were also found at the $100 \%$ stiffness levels. For Golfer-Fast, the peak of the 'clubhead speed - penalties' curve does not quite reach the 'clubhead speed' curve (Fig. 3.1.3.3). This happened because during the optimal swing, at the $100 \%$ level of shaft stiffness, a small sum of penalties still accumulated. The penalties resulted from the clubface being approximately half of a degree away from pointing directly down the target line. 


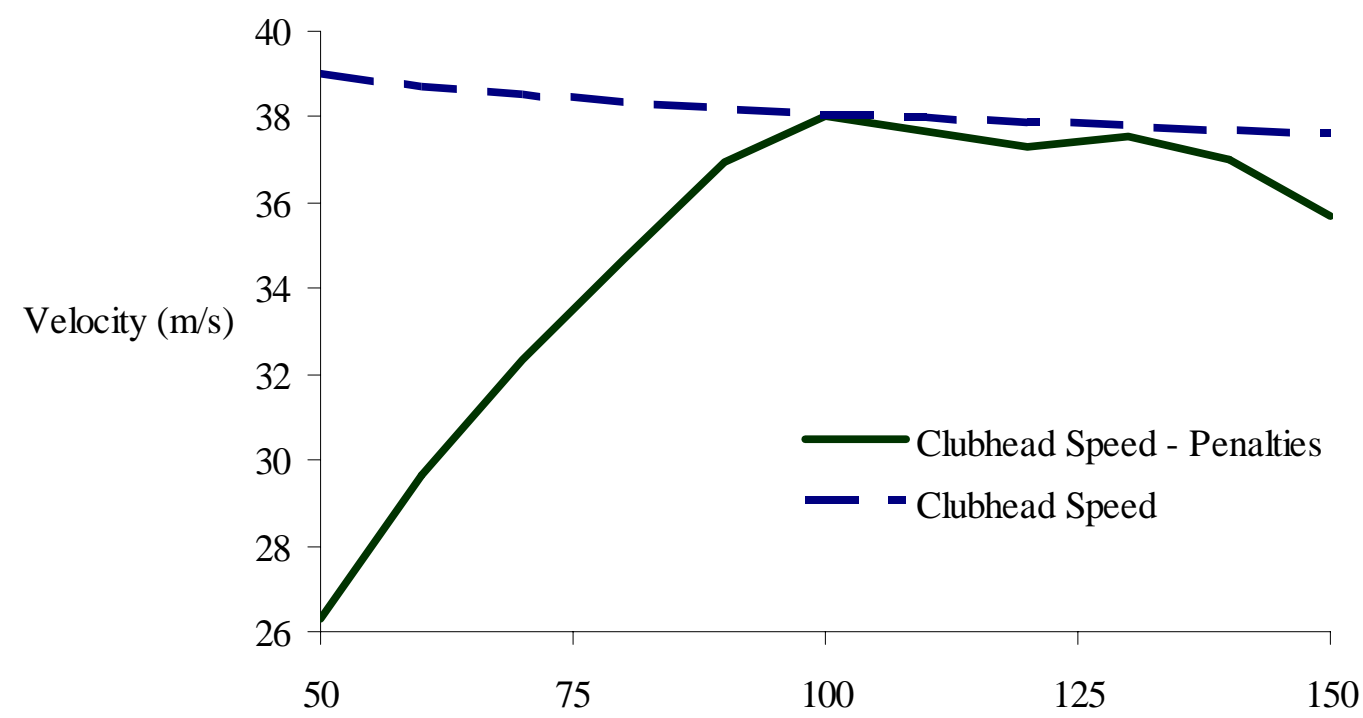

Percentage of Club-Regular Shaft Stiffness (\%)

Figure 3.1.3.2 Resulting clubhead speed and clubhead speed minus penalties over a complete range of shaft stiffness for a single swing pattern from Golfer-Slow.

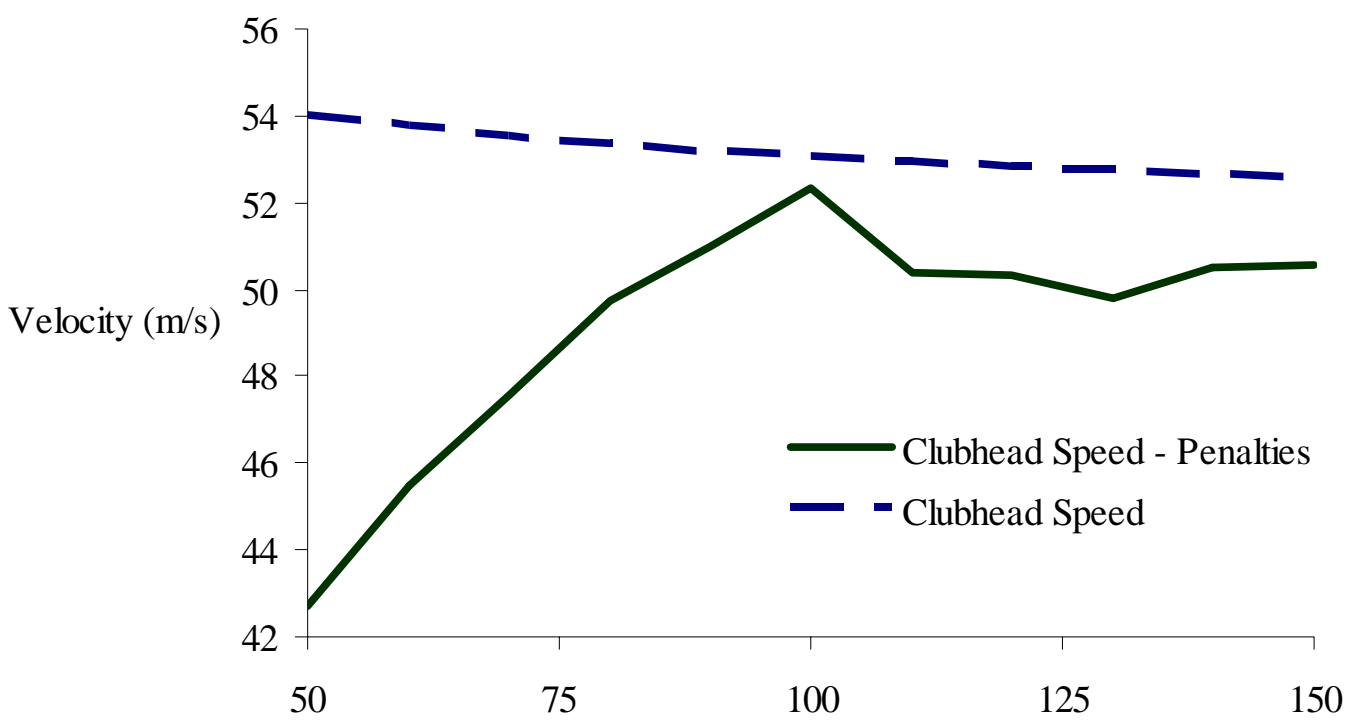

Percentage of Club-Regular Shaft Stiffness (\%)

Figure 3.1.3.3 Resulting clubhead speed and clubhead speed minus penalties over a complete range of shaft stiffness for a single swing pattern from Golfer-Fast. 


\subsection{Simulated Thought Experiments Using the Golfer-Club Model}

\subsubsection{Use of a Perfectly Rigid Shaft}

The three golfer models generated less clubhead speed at impact when optimized with rigid shafts, than when optimized with non-rigid shafts (Table 3.2.1.1). As the golfer model's ability to generate torque increased, the difference in clubhead speed between the rigid shaft simulation and non-rigid shaft simulations increased. The Golfer-Fast model showed the largest discrepancy $(1.87 \mathrm{~m} / \mathrm{s})$, while the Golfer-Slow model showed the least difference $(0.15 \mathrm{~m} / \mathrm{s})$ between clubhead speeds at impact.

Table 3.2.1.1 Clubhead speeds attained with rigid shafts and the highest clubhead speeds attained with any of the three flexible shafts for each of the three golfer models.

\begin{tabular}{|c|c|c|c|c|c|c|c|c|c|}
\hline \multicolumn{1}{|c|}{ Swing Speed Level } & \multicolumn{3}{|c|}{ Golfer-Slow } & \multicolumn{3}{c|}{ Golfer-Medium } & \multicolumn{3}{c|}{ Golfer-Fast } \\
\hline Shaft Stiffness Level & $\begin{array}{l}\text { Club } \\
\text { Rigid }\end{array}$ & $\begin{array}{l}\text { Club } \\
\text { Reg. }\end{array}$ & Diff. & $\begin{array}{l}\text { Club } \\
\text { Rigid }\end{array}$ & $\begin{array}{l}\text { Club } \\
\text { Stiff }\end{array}$ & Diff. & $\begin{array}{l}\text { Club } \\
\text { Rigid }\end{array}$ & $\begin{array}{l}\text { Club } \\
\text { Flex. }\end{array}$ & Diff \\
\hline Clubhead Speed (m/s) & 37.90 & 38.05 & 0.15 & 43.51 & 45.04 & 1.53 & 51.09 & 52.96 & 1.87 \\
Kick Velocity (m/s) & 0 & 4.84 & 4.84 & 0 & 6.88 & 6.88 & 0 & 9.65 & 9.65
\end{tabular}

The difference in clubhead speed performance between rigid and non-rigid shafts indicates that the non-rigid shafts were able to store and then subsequently release energy during the swing. However, these differences are relatively small in comparison to the kick velocities attained at impact during the non-rigid shaft simulations (Table 3.2.1.1). For example, Golfer-Medium showed a $1.53 \mathrm{~m} / \mathrm{s}$ increase in clubhead with Club-Stiff over Club-Rigid. However, the Golfer-Medium\Club-Stiff combination resulted in a kick velocity of $6.88 \mathrm{~m} / \mathrm{s}$ at impact. Superficially, this suggests that the shaft flexibility resulted in an increased clubhead speed of $6.88 \mathrm{~m} / \mathrm{s}$ over a rigid shaft. However, this statement is only true if the non-rigid shaft is replaced by a rigid shaft and 
no change in the kinematics of segments proximal to the rotational spring-damper elements occurs. In reality, as well as in the model, the kinematics of the proximal segments will change when the club is replaced. This is because the acceleration of the system is not predetermined, but rather it is dictated by the forces and torques acting on the segments. Since the two-segment golfer model dynamically interacts with the foursegment club model, a rigid club will result in different golfer kinematics than a nonrigid club. Taken together, these comments imply that, at impact, the angular velocity of the most proximal club segment will be less with a non-rigid shaft as compared to a rigid shaft. The angular velocities of the most proximal club segment, about an axis perpendicular to the swing plane, for Golfer-Medium with Club-Stiff and GolferMedium with Club-Rigid supported this view (Fig. 3.2.1.1). 


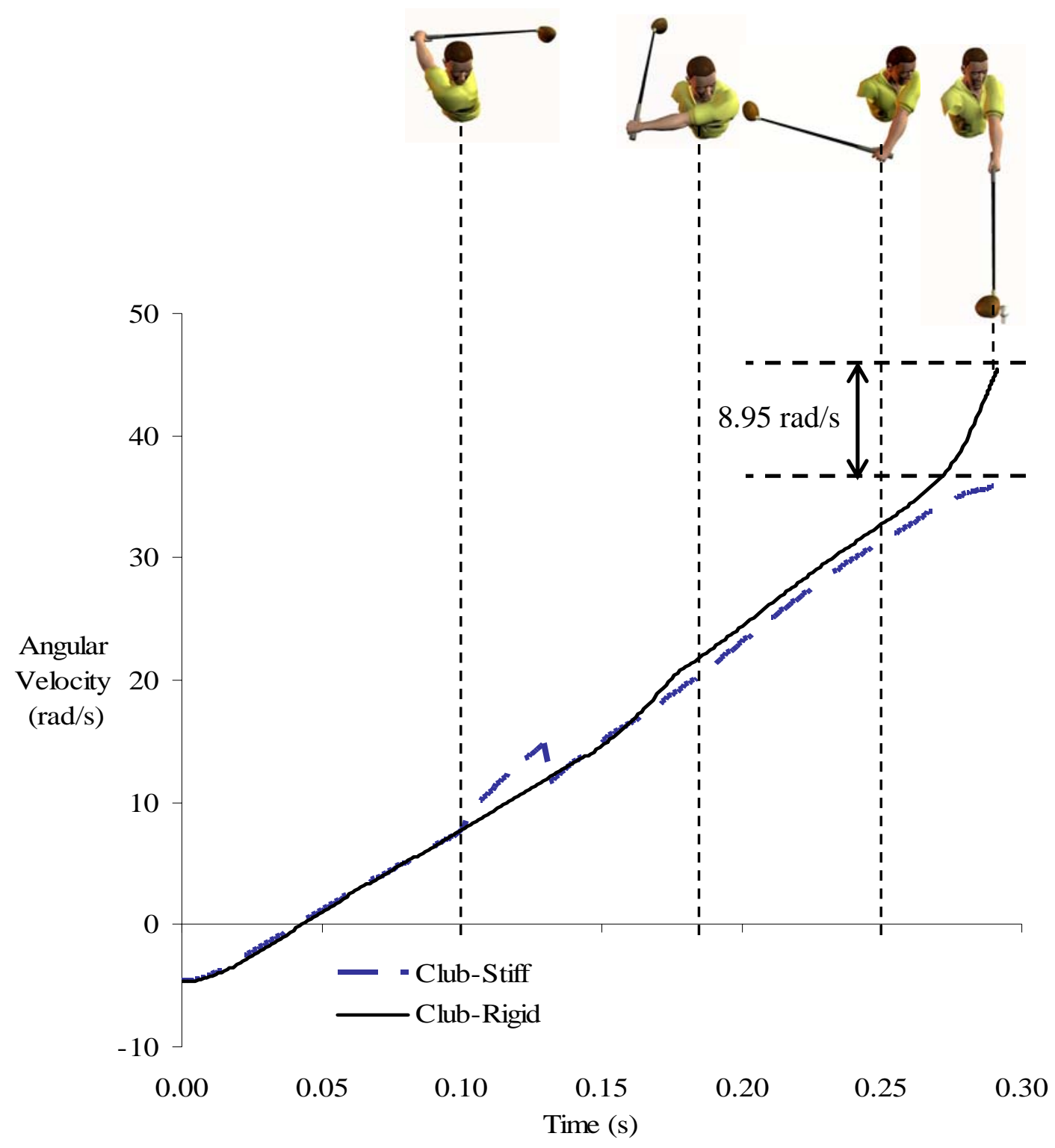

Figure 3.2.1.1 Angular velocity of the most proximal club segment for the optimized simulations of Golfer-Medium with Club-Stiff and Golfer-Medium with Club-Rigid.

At impact, the most proximal club segment of Club-Rigid had a higher angular velocity (+8.95 rad/s) than that of Club-Stiff (Fig. 3.2.1.1). This higher angular velocity provided the potential for a higher clubhead speed at impact. However, the added kick velocity $(6.88 \mathrm{~m} / \mathrm{s})$ provided by the non-rigid shaft resulted in Club-Stiff possessing a greater clubhead speed $(+1.53 \mathrm{~m} / \mathrm{s})$ at impact. The torque provided by the rotational 
spring-damper elements resulted in the added kick velocity. However, just as the torque increases the clubhead velocity, it conversely decreases the angular velocity of the most proximal club segment.

\subsubsection{Repositioning the Clubhead Center of Mass}

Repositioning the center of mass of the clubhead altered the behaviour of the shaft by an amount large enough to have a meaningful effect on clubhead orientation at impact. The effects of repositioning the center of mass along the two axes of shaft deflection ( $x, y$, see Fig. 1.2.1.1) were independent of one another. For example, changing the position of the center of mass along the $x$ axis only affected shaft deflection in the toe-up/toe-down directions and had no measurable influence on lead/lag deflection. Similarly, changing the position of the center of mass along the $y$ axis only affected shaft deflection in the lead/lag directions and had no measurable influence on toe-up/toe-down deflection. For this reason toe-up/toe-down deflections are presented separately in Figure 3.2.2.1 to demonstrate the effects of changing the center of mass along the $x$ axis. Lead/lag deflections are presented in Figure 3.2.2.2 to demonstrate the effects of repositioning the center of mass along the $y$ axis. 


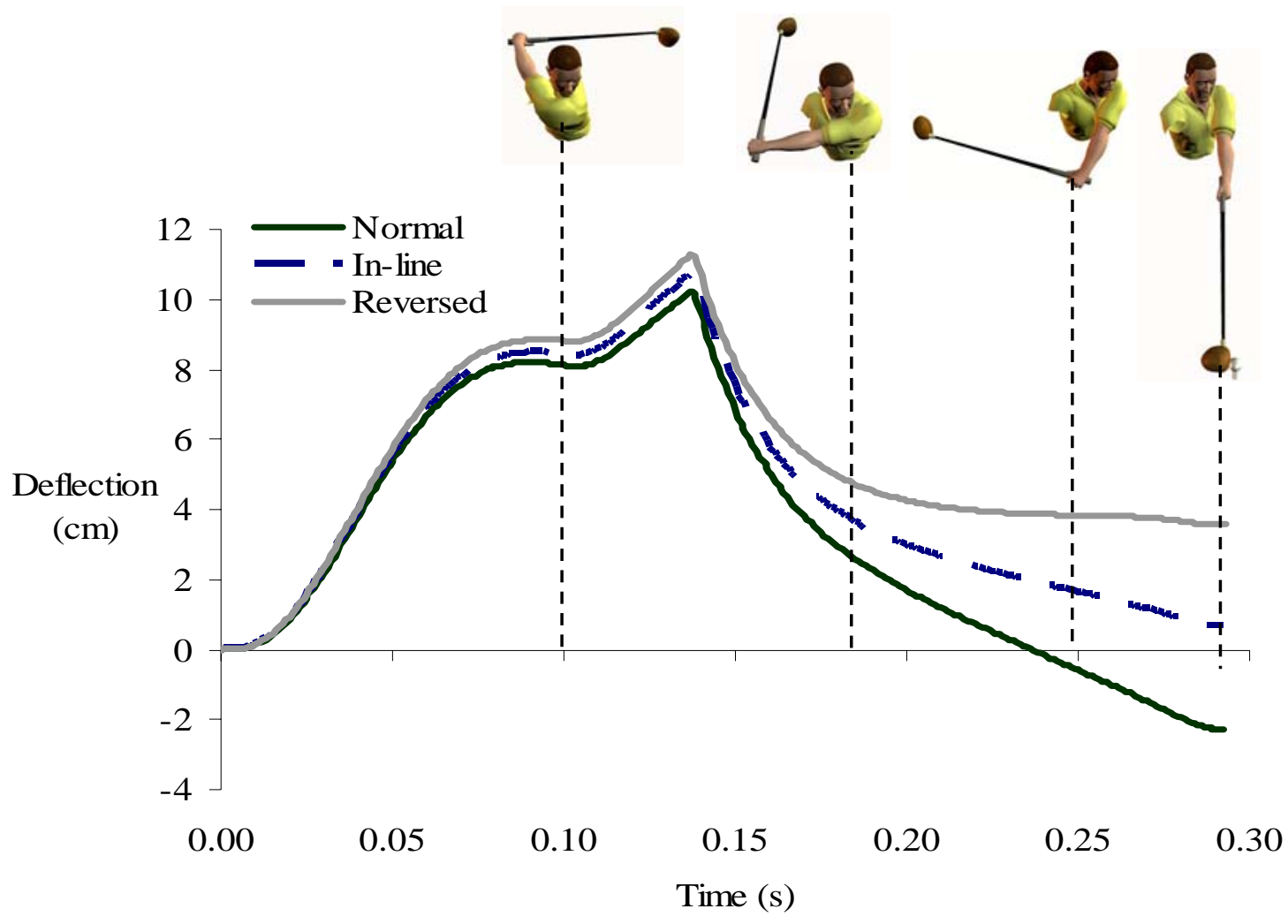

Figure 3.2.2.1 Toe-up/toe-down deflections for three different clubhead center of mass positions. Golfer-Medium/Club-Regular was used for all three conditions. Normal refers to a standard driver that has the center of mass located in the toe-up direction relative to the shaft insertion point. In-line refers to having the center of mass collinear with the shaft. Reversed refers to having the center of mass in the toe-down direction relative to the shaft.

During the first half of the downswing, tangential forces, not radial, exerted the greatest influence on the toe-up/toe-down deflections. This can be reasoned because all three conditions showed very similar deflection patterns and all had peak toe-up deflections of approximately the same magnitude $(10 \mathrm{~cm})$ during the first half of the downswing (Fig 3.2.2.1). However, as impact approached radial force became an important factor in determining shaft deflection in the toe-up/toe-down direction. As expected by theory, the Normal center of mass position condition resulted in a toe-down deflection $(-2.26 \mathrm{~cm})$ at impact. The In-line condition approached zero, but finished 
with a small toe-up deflection $(0.69 \mathrm{~cm})$ at impact. Reversing the center of mass position along the $x$ axis resulted in a relatively large toe-up deflection $(3.60 \mathrm{~cm})$ at impact. If radial force completely dictated toe-down deflection at impact, then the Inline condition would have resulted in a toe-up deflection of $0 \mathrm{~cm}$ at impact not $0.69 \mathrm{~cm}$. This toe-up deflection at impact for the In-line condition was a residual effect of the large tangential forces that occurred earlier in the downswing. The same effect is also evident in the other two conditions. For example, if $0.69 \mathrm{~cm}$ is subtracted from all three conditions $(-1.26,0.69,+3.60)$, the result is a near symmetrical relationship around zero $(-2.95,0,+2.91)$. This systematic shift suggests that the tangential forces applied earlier in the downswing had a similar effect on all three conditions. 


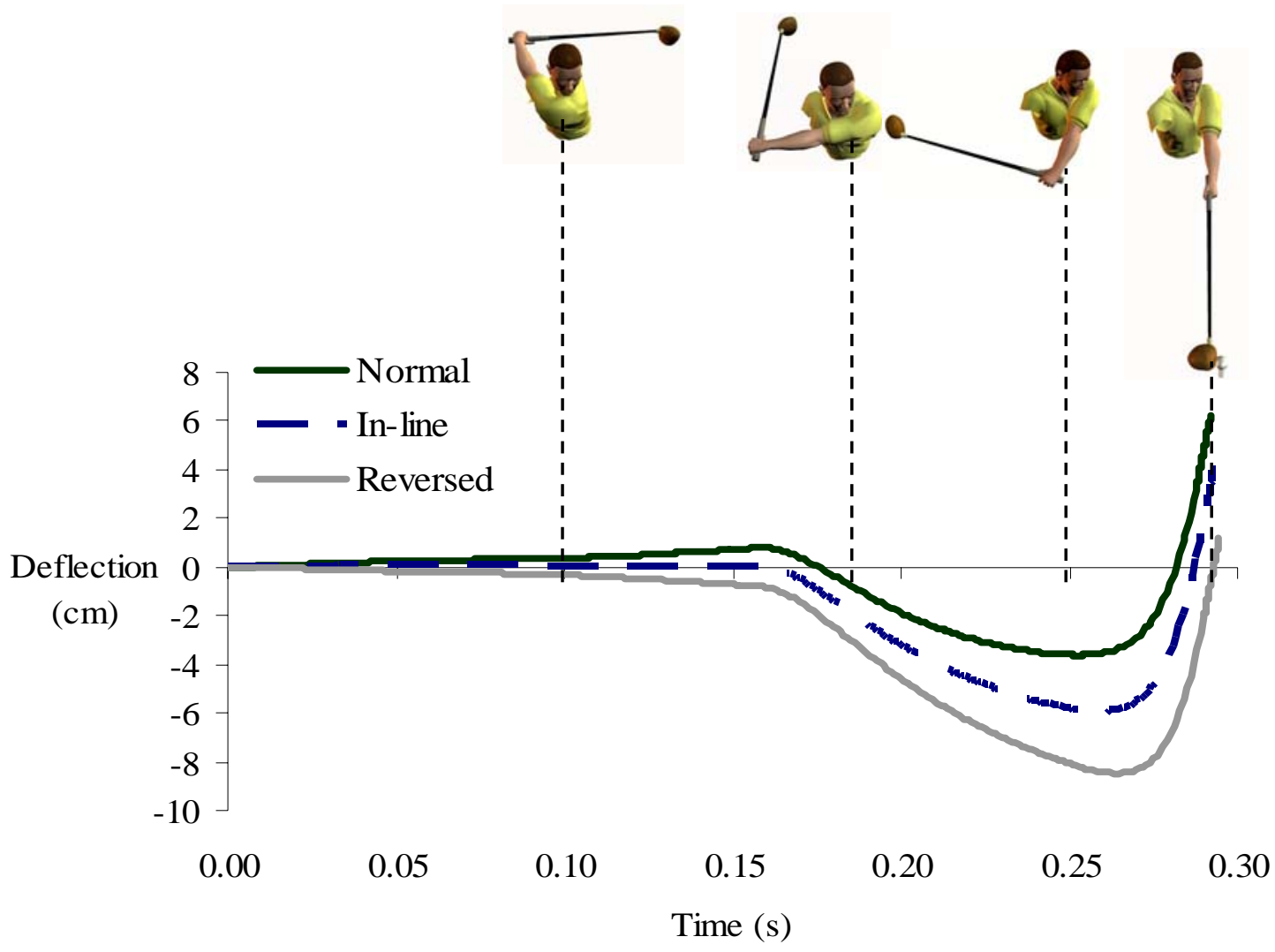

Figure 3.2.2.2 Lead/lag deflections for three different clubhead center of mass positions. Golfer-Medium/Club-Regular was used for all three conditions. Normal refers to a standard driver that has the center of mass located in the lag direction relative to the shaft insertion point. In-line refers to having the center of mass collinear with the shaft. Reversed refers to having the center of mass in the lead direction relative to the shaft.

For the first half of the downswing, the influence that radial force had on lead/lag deflection is evident (Fig. 3.2.2.2). In the Normal condition, the effect was to pull the center of mass in line with the shaft which resulted in the clubhead moving into a leading position. Reversing the position of the center of mass had an equal and opposite effect, as the clubhead was pulled into a lagging position. The In-line condition served as a verification of the radial force influence; when the center of mass 
was collinear with the shaft no bending in the lead/lag direction occurred during the first half of the downswing. Radial force had a clear influence on lead/lag deflection at impact. The Normal condition showed the greatest lead deflection at impact $(6.25 \mathrm{~cm})$ followed by the In-line condition $(3.97 \mathrm{~cm})$ and then by the Reversed condition (1.21 $\mathrm{cm})$. However, since all conditions resulted in lead deflections at impact, there was clearly another factor at work. That factor was likely the momentum generated by the torque from the rotational springs recovering the shaft from its lagging position just prior to impact. Since all conditions showed lag deflections from approximately $0.17 \mathrm{~s}$ to $0.27 \mathrm{~s}$, it seems apparent that tangential forces were the cause of this lagged state. It seems as though both tangential and radial forces play important roles in the lead/lag deflections during the downswing and that it is difficult to tease apart their influences.

\subsubsection{Removal and Isolation of Radial Force}

Complete removal of the radial force component from the optimized swing of Golfer-Medium with Club-Regular had a simple effect on shaft deflection in both the lead/lag and toe-up/toe-down directions (Fig. 3.2.3.1). The pattern of shaft deflection remained very similar when radial force was removed. Only the magnitude of deflection was affected, and the difference in magnitude between the conditions increased as impact approached. This was logical considering that radial force increased as impact approached (Fig. 3.2.3.3). Lead deflection at impact remained positive (4.72 $\mathrm{cm})$ but was reduced from its value under normal conditions $(6.25 \mathrm{~cm})$. Toe-down deflection at impact remained negative $(-1.02 \mathrm{~cm})$ but was also reduced in magnitude in 
comparison to the normal condition $(-2.26 \mathrm{~cm})$. This reduction seems reasonable when the effect of radial force on shaft deflection was isolated (Fig. 3.2.3.2).

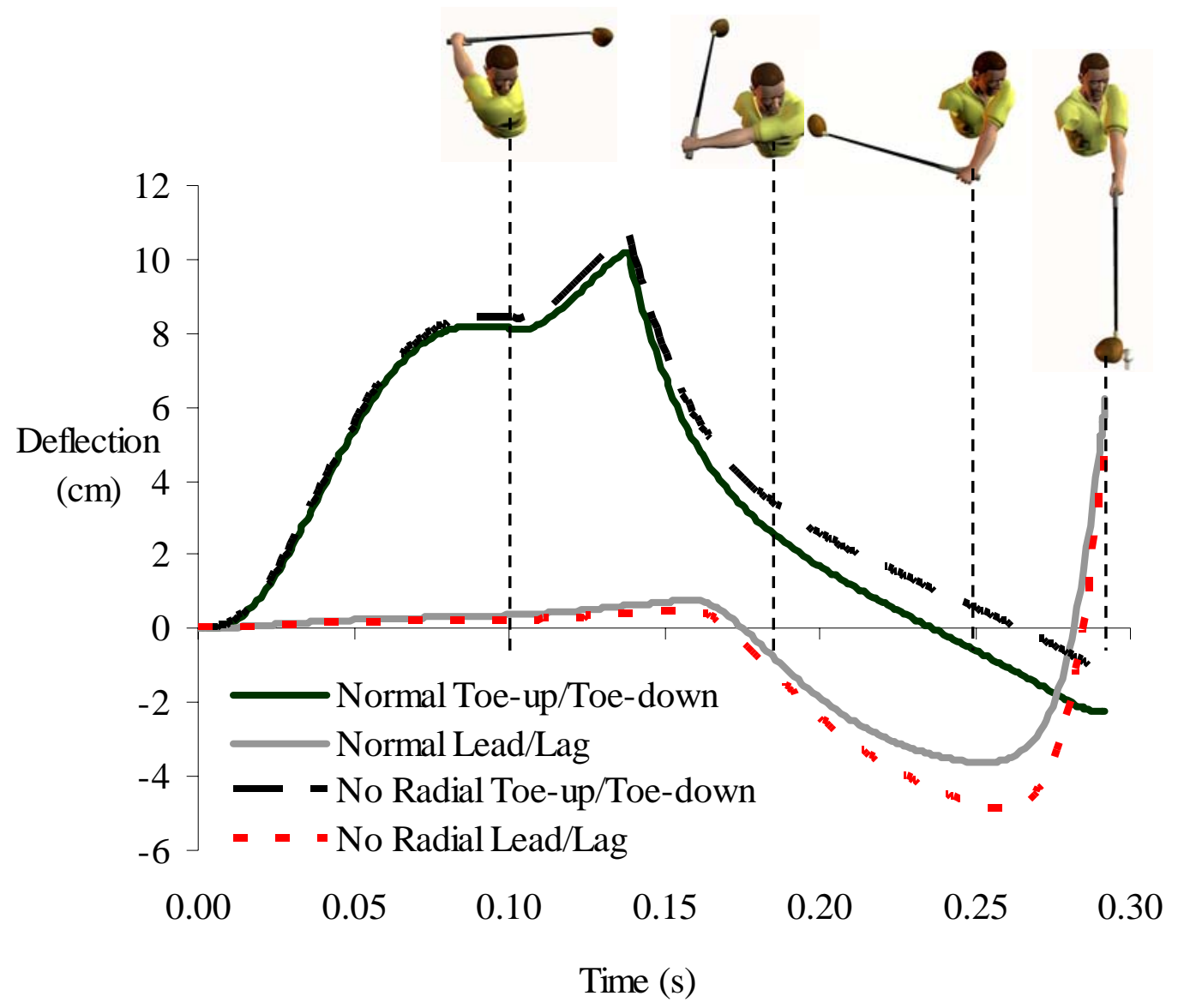

Figure 3.2.3.1 Comparison of shaft deflections between the simulated swing of GolferMedium with Club-Regular and the same swing with radial force removed.

Care should be taken in a comparison of the results from section 3.2.3 and 3.2.4. On the surface, one might predict that moving the clubhead's center of mass in line with the shaft would have the same effect on shaft deflection as removing radial force completely. However, these two conditions will not necessarily produce identical results. Consider a shaft that is considerably deflected in the toe-up direction one third 
of the way into the downswing (Fig. 3.2.3.1). Even if the clubhead center of mass was originally in the In-line position, radial force would still exert an influence because of the clubhead's current offset position. This influence would affect the dynamics of shaft deflection for the rest of the swing. If radial force was removed completely, this would not be the case.

When acting in isolation of all other forces and torques, radial force produced nearly identical patterns of shaft deflections about both axes of shaft bending (Fig. 3.2.3.2). Due to the off-set position of the clubhead's center of mass, the shaft was gradually pulled into its maximum leading position $(1.22 \mathrm{~cm})$ at impact. Similarly, the shaft was gradually pulled into its maximum toe-down position $(-1.33 \mathrm{~cm})$ at impact when radial force acted as the lone contributor to shaft deflection. The club showed more deflection in the toe-down direction than in the lead direction because the clubhead center of mass was more off-set along the $x$ axis $(5.2 \mathrm{~cm})$ than the $y$ axis $(4.7$ cm) (Table 2.1.1.1). 


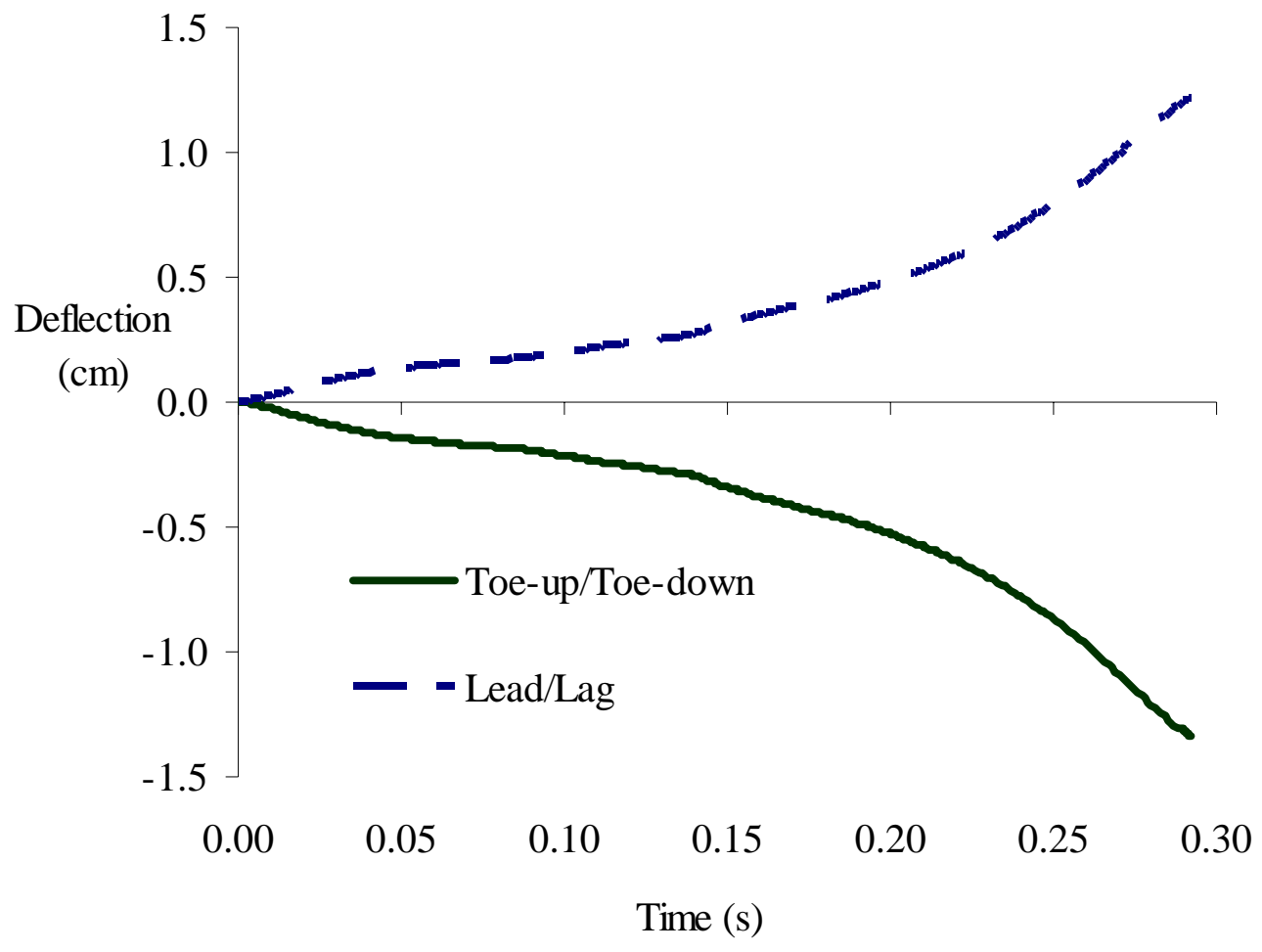

Figure 3.2.3.2 Shaft deflection when radial force acted in isolation of all other forces and torques supplied by Golfer-Medium to Club-Regular during the optimized swing.

Radial force increased in value up to a point just before impact. This is likely related to the fact that swing adjustments have to be made in order to achieve the proper impact positions with the golfer model. Radial force peaked 0.01 seconds before impact at a value of $456 \mathrm{~N}$ before dropping to $413 \mathrm{~N}$ at impact with the ball (Fig. 3.2.3.3). Aside from a negligible gravitational component, the radial force component supplied by the golfer is required to maintain circular motion of the club during the downsing. For a given tangential velocity, the amount of force required to maintain circular motion decreases as the radius associated with that circular motion increases. With regards to the model, over the final 0.01 seconds the effective radius associated with the club's 
circular motion increases. Essentially the motion of the club's center of mass becomes more linear due to the combined motion of the torso, and arm segments near impact. For this reason, radial force decreased in magnitude during the simulated downswing.

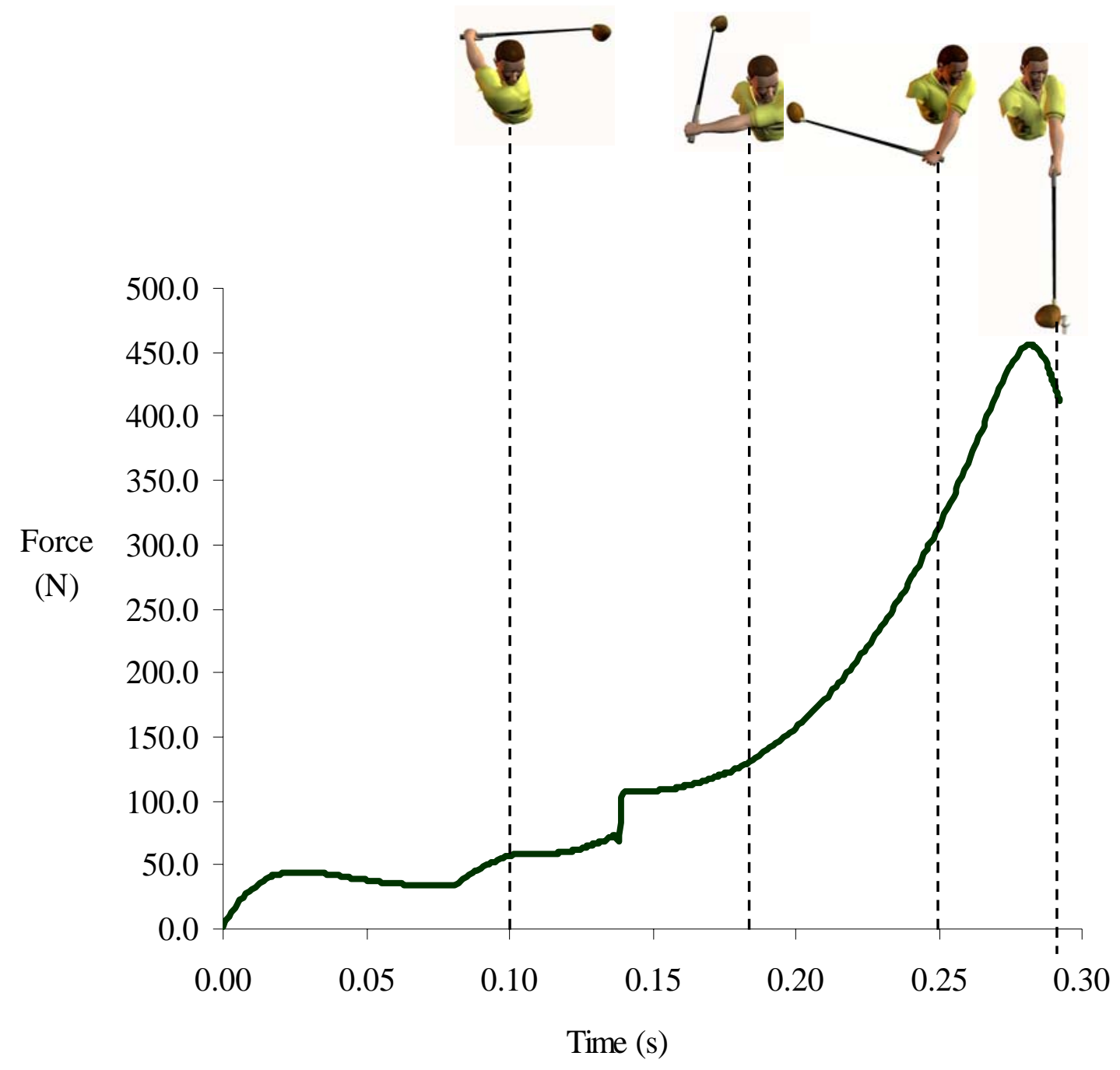

Figure 3.2.3.3 Radial force acting along the $z$ axis of the most proximal club segment during the optimized swing of Golfer-Medium with Club-Regular. 


\subsubsection{Effect of Shaft Deflection on Clubhead Orientation}

A clear linear relationship existed between the amount of lead deflection and the resulting dynamic loft (Fig. 3.2.4.1). For each centimeter of lead deflection, dynamic loft increased by approximately $0.8^{\circ}$. Toe-down deflection had relatively no influence on dynamic loft. Toe-down deflection exerted its greatest influence when accompanied by the maximum amount of lead deflection, yet still only increased dynamic loft by $0.1^{\circ}$ over the entire range of toe-down deflection values.

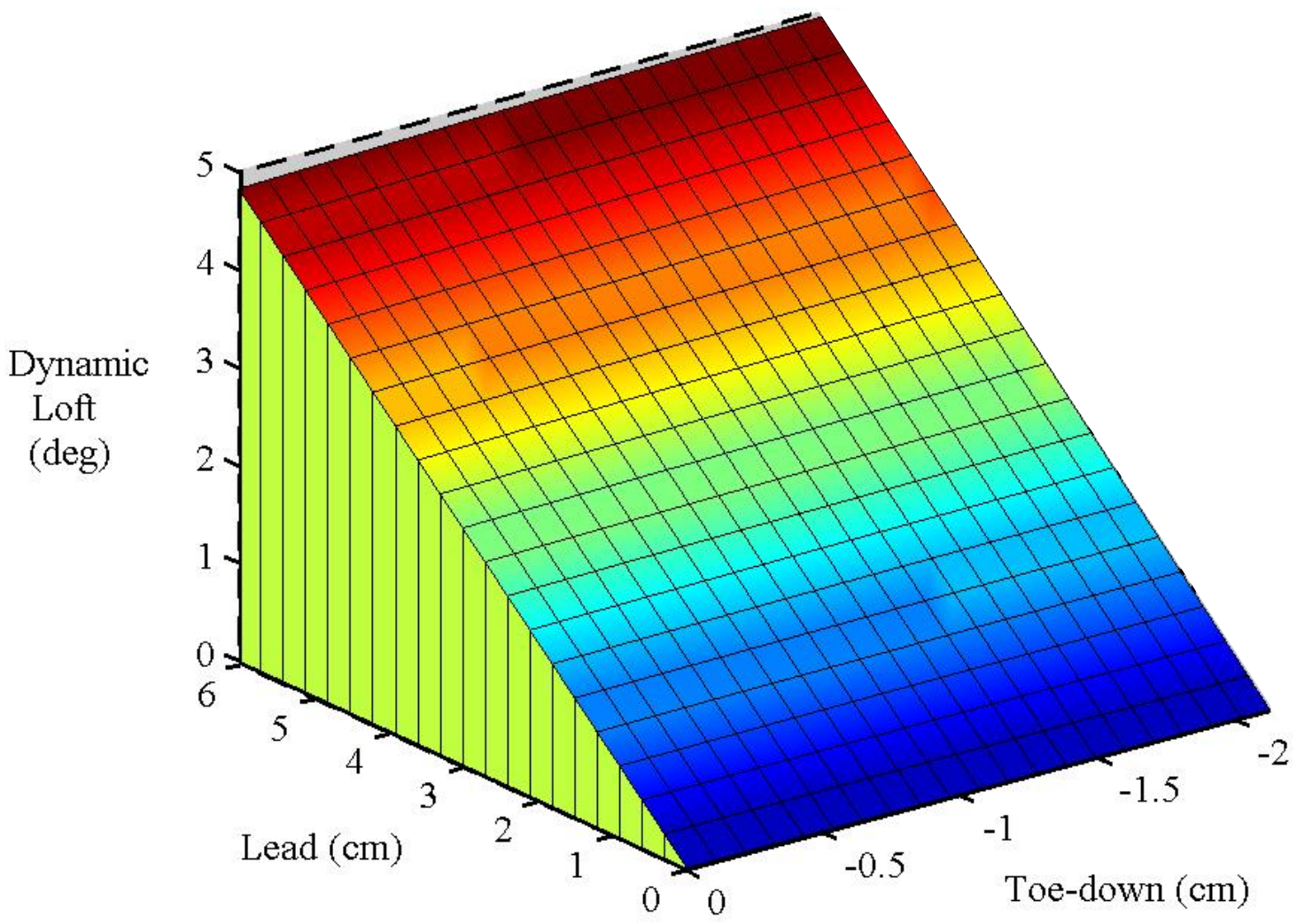

Figure 3.2.4.1 Three dimensional plot of dynamic loft as a function of both lead and toe-down deflections. 
Lead deflection exerted a greater influence on dynamic close than toe-down deflection. However, toe-down deflection did have more of an effect on dynamic close than on dynamic loft (Fig. 3.2.4.2). Lead deflection showed a similar linear relationship with dynamic close in comparison to its relationship with dynamic loft. For every centimeter increase in lead deflection, dynamic close increased by approximately $0.7^{\circ}$. Toe-down deflection served to reduce the influence of lead-deflection on dynamic close over the entire range of lead deflection values. For example at the highest amount of lead deflection graphed, there was an approximately $1^{\circ}$ change in dynamic close over the range of toe-down deflection values.

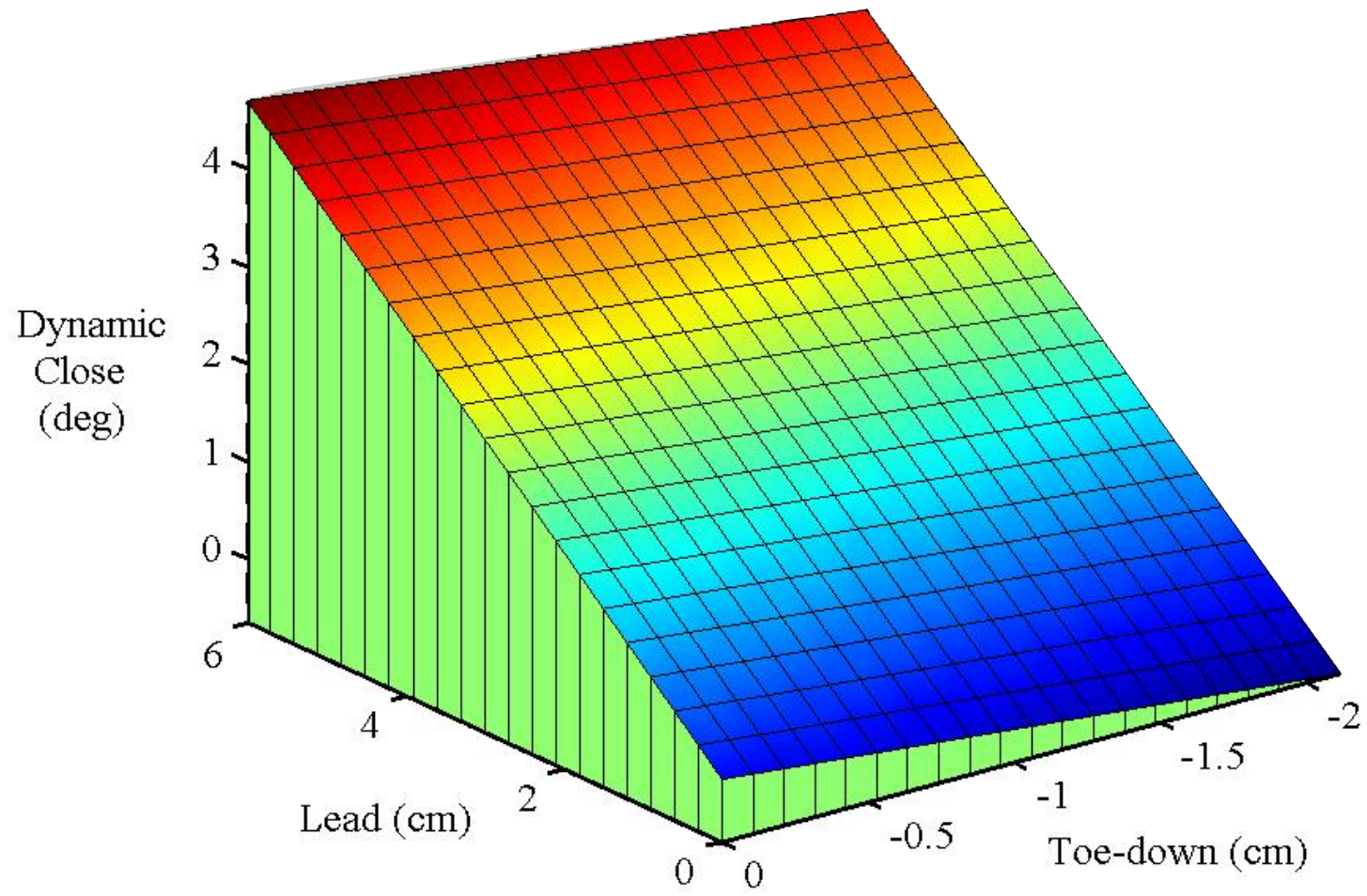

Figure 3.2.4.2 Three dimensional plot of dynamic close as a function of both lead and toe-down deflections. 


\subsection{Model Validation}

\subsubsection{External Validity}

\subsubsection{Live Golfer Testing}

There was a trend for lead deflection at impact to increase in magnitude as shaft stiffness decreased (Table 3.3.1.1.1). This finding supports the results for the lead deflections measured during the simulations. For three out of the four live golfers tested, Club-Flexible had the most lead deflection at impact followed by Club-Regular and then by Club-Stiff. The other right-handed golfer, Live Golfer 3, produced the most lead deflection with Club-Flexible, but Club-Stiff showed slightly more lead deflection $(0.76 \pm 0.57 \mathrm{~cm})$ than Club-Regular $(0.44 \pm 0.21 \mathrm{~cm})$. Some inconsistency in the live golfer testing results is to be expected. Williams and Sih (2002) reported an average within subject standard deviation of $2.94^{\circ}$ for loft angle with a single driver which is a strong indication that lead deflection was also highly variable.

There appeared to be no relationship between lead deflection and clubhead speed at impact for the live golfer testing. For example, Live Golfer 4, generated the slowest average clubhead speed with Club-Flexible $(39.13 \pm 0.67 \mathrm{~m} / \mathrm{s})$, yet produced the greatest average lead deflection $(3.64 \pm 0.10 \mathrm{~cm})$ with the same club. Further, while swinging Club-Regular, both Live Golfer 3 and Live Golfer 4 had the same average clubhead speeds at impact $(39.63 \pm 2.15 \mathrm{~m} / \mathrm{s}, 39.63 \pm 2.02 \mathrm{~m} / \mathrm{s})$, yet produced the lowest $(0.44 \pm$ $0.21 \mathrm{~cm})$ and highest $(2.90 \pm 0.61 \mathrm{~cm})$ average lead deflections respectively with the same club. 
The lack of a relationship between clubhead speed and lead deflection appears not to support the simulation data. However, the simulation data were derived from optimized swings in which the club and golfer were perfectly matched to generate the highest clubhead speed possible. Each optimized simulation also showed relatively similar patterns of muscle torque generation which were responsible for both the forces applied to the club and the generation of clubhead speed. Shaft deflection is directly influenced by the forces applied to the club, and not essentially by the velocity that results from those forces (Butler \& Winfield, 1994; Mather \& Cooper, 1994). Radial force is responsible for maintaining the circular motion of the club and approximately half of the resulting deflections at impact. For a given clubhead speed at impact, the amount of radial force required to maintain a particular curved motion will always be nearly the same. Therefore, the resulting magnitudes of shaft deflection due to that radial force will also be the same. The tangential forces are responsible for the generation of clubhead speed, the majority of the shaft deflection during the first $75 \%$ of the downswing, and at least half of the resulting deflections at impact. However, very different patterns of tangential force can result in the same clubhead speed but with very different patterns of shaft deflections during the swing and at impact. The live golfers were probably employing relatively inconsistent and sub-optimal (compared to the model) muscle coordination strategies. Therefore, it is likely that the live golfers, due to differing muscle coordination strategies, generated different patterns of tangential forces. These varying tangential force applications would be responsible for the lack of a clubhead speed - shaft deflection relationship. Further, the clubhead speeds generated from the computer simulation results ranged from 38 to $53 \mathrm{~m} / \mathrm{s}$, while the live golfers 
had a range of 37 to $43 \mathrm{~m} / \mathrm{s}$. This smaller range of clubhead speeds would also make it difficult to detect differences in shaft deflection variables as a function of clubhead speed.

While swinging the right-handed Club-Regular, lead deflections hovered around zero $(-0.11 \pm 0.08 \mathrm{~cm})$ for the left-handed golfer. It was likely that the reversed position of the clubhead's center of mass along the $y$ axis resulted in the lack of lead deflection at impact for the left-handed golfer despite a relatively high average clubhead speed (45.07 $\pm 0.24 \mathrm{~m} / \mathrm{s})$. This finding is in agreement with the simulation results generated when the clubhead center of mass position was reversed along the $y$ axis for the GolferMedium/Club-Regular pairing. This certainly suggests that radial force plays an important in role in shaft deflection at impact. However it also implies, as did the simulation results from section 3.2.2, that radial force does not completely dictate the magnitude shaft deflection at impact. If radial force was completely dictating shaft deflection at impact, then the shaft would be deflected by a larger amount in the lag direction, and not just in a relatively neutral position $(-0.11 \pm 0.08 \mathrm{~cm})$.

Two additional points should be made regarding the results from the Perspective 1 highspeed video data. First, with the exception of the left-handed golfer, every swing with every club resulted in a lead deflection at impact, as did the simulation results. This is a strong indication that the golfer model possesses a sufficient level of external validity. Second, none of the golfers demonstrated consistently higher clubhead speeds with any single club. Compared to the other participants, Live Golfer 3 had the largest difference in average clubhead speed between the two clubs he performed the best with. Live Golfer 3 had his highest average clubhead speed with Club-Flexible (41.7 \pm 1.73 
cm) and his second highest average with Club-Stiff $(39.10 \pm 3.29 \mathrm{~cm})$. The resulting difference was $2.60 \mathrm{~m} / \mathrm{s}$, yet the large standard deviations associated with both averages confirm a lack of stability in club performance and suggest that a meaningful difference did not exist.

Table 3.3.1.1.1 Lead deflections and clubhead speeds at impact as determined from Perspective 1. Values are the average and standard deviations from three swings. Lead is presented in centimetres, and clubhead speed is given in meters per second.

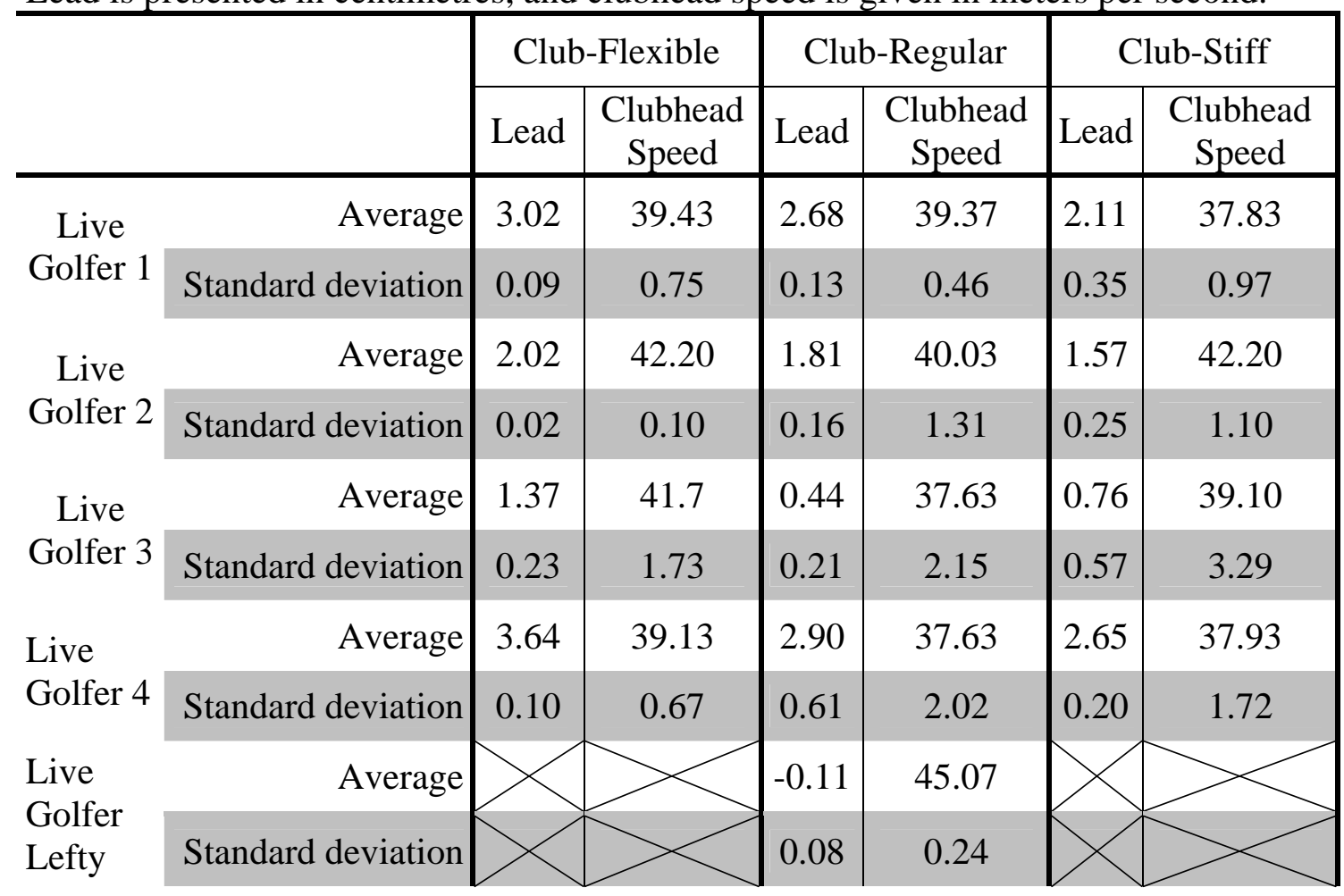

The shaft deflection curve shown for Live Golfer 4 (Fig. 3.3.1.1.1), as captured from Perspective 1, needs to be interpreted differently than the shaft deflection curves reported from the simulations. The curve should be interpreted as follows. Relative to a reference frame fixed in the swing plane, if the clubhead is lagging behind the hands, then the deflection will be negative. However, if the clubhead is leading ahead of the 
hands, then the deflection will be positive. Therefore, the toe-up deflection of the shaft at the start of the downswing was recorded as negative because the clubhead was trailing the hands relative to a reference frame fixed in the swing plane. All golfers with all three clubs demonstrated the same general pattern of shaft deflection during the downswing. That general pattern was negative deflection for $80-90 \%$ of the downswing followed by positive deflection over the final $10-20 \%$. Larger negative shaft deflections were recorded as shaft stiffness decreased. These results are in agreement with the simulation results and indicate that the mathematical model was able to sufficiently represent the pattern of shaft bending during the downswing. The magnitudes of shaft deflection measured during the live golfer testing were consistently less during all points in the downswing. This was predictable and expected based on the differences in the methods used to calculate shaft deflection in the live golfer versus simulation experiments described in section 2.4.1. 


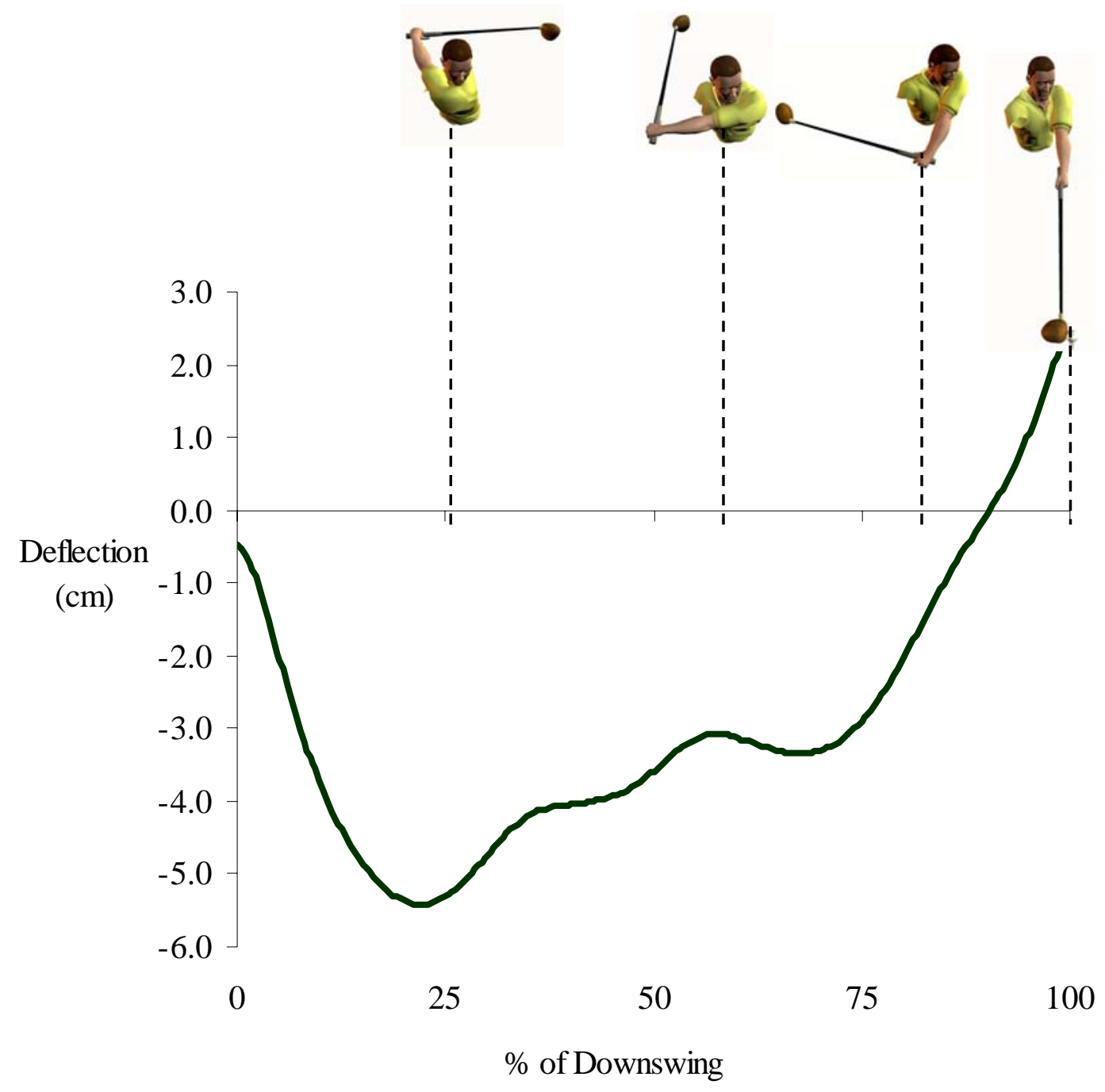

Figure 3.3.1.1.1 In-plane shaft deflection during the downswing of Live Golfer 4 with Club-Regular as captured from Perspective 1.

The relatively large variability within conditions compared to the small difference between conditions makes it difficult to infer any trends in the toe-up/toedown deflection data analyzed from Perspective 2 (Table 3.3.1.1.2). This was similar to the toe-down deflection data gathered from the simulations in terms of the variability and lack of a clear pattern across levels of shaft stiffness even with optimized swings. 
Every live golfer-swing analyzed clearly showed toe-down deflections values at impact which is in support of the simulation results.

Table 3.3.1.1.2 Toe-down deflections at impact as determined from Perspective 2. Values are given in centimetres, and are the average and standard deviations for three swings.

\begin{tabular}{|c|c|c|c|c|}
\hline & & Club-Flexible & Club-Regular & Club-Stiff \\
\hline \multirow{2}{*}{$\begin{array}{l}\text { Live } \\
\text { Golfer } 2\end{array}$} & Average & -1.94 & -2.55 & -2.84 \\
\hline & Standard deviation & 1.02 & 1.10 & 0.63 \\
\hline \multirow{2}{*}{$\begin{array}{c}\text { Live } \\
\text { Golfer } 3\end{array}$} & Average & -1.66 & -2.14 & -1.63 \\
\hline & Standard deviation & 0.90 & 0.65 & 0.55 \\
\hline \multirow{2}{*}{$\begin{array}{c}\text { Live } \\
\text { Golfer } 4\end{array}$} & Average & -3.58 & -3.79 & -3.42 \\
\hline & Standard deviation & 0.77 & 0.46 & 0.94 \\
\hline
\end{tabular}

\subsubsection{Error and Reliability of Live Golfer Testing}

The process used to gain an estimate of the error associated with the live golfer testing revealed an average length of the first club segment of $27.4 \mathrm{~cm}$ compared to the true length of $27.2 \mathrm{~cm}$. The average total range $(1.8 \mathrm{~cm})$, average standard deviation $(0.36 \mathrm{~cm})$ and average coefficient of variation (1.4\%) across all swings demonstrated that the error associated with the video and digitizing process was at an acceptable level. This level was considered acceptable because when the error was factored into the live golfer results, the conclusions remained the same. The reliability calculation revealed that if shaft deflection was measured twice using the same raw video data, then 95\% of the difference scores between measurements would fall within $\pm 0.6 \mathrm{~cm}$. This level of 
reliability does not affect the interpretation of the live golfer testing results presented above.

\subsubsection{Swing Plane Comparison}

Two allowances were made in the development of the model used in this thesis which permitted the swing plane values throughout the downswing to follow the same general pattern determined by Coleman and Rankin (2005). The first allowance was to constrain the arm to move in a plane that was $20^{\circ}$ steeper than the plane on which the torso rotated. This inherently resulted in the swing plane becoming steeper as impact approached. The second modification was to permit the arm segment to rotate about its longitudinal axis. Implementing this degree of freedom into the model permitted the potential for swing plane variations in either direction during the downswing. Coleman and Rankin showed that, for a 5 iron swung by a professional golfer (Participant 1 in their study), the swing plane showed a slight increase in angle before peaking at approximately $138^{\circ}$ and then steadily decreasing down to an angle of approximately $103^{\circ}$ at impact. The swing plane angle was measured from the right horizontal, not the left horizontal as depicted in Figure 2.1.1.2 of this thesis. Therefore, Coleman and Rankin showed that the swing plane for Participant 1 increased in steepness by approximately $35^{\circ}$ throughout the downswing. The swing plane angle for the GolferMediumlClub-Regular simulation also increased in steepness by approximately $35^{\circ}$ as it decreased from a maximum angle of $165^{\circ}$ to an angle of $130^{\circ}$ at impact (Fig. 3.3.1.3.1).

The swing plane angle followed the same general pattern as presented by Coleman and Rankin (for Participant 1 who had a 0 handicap), but is consistently flatter at all points 
during the swing. Due to the added length of the driver over a 5 iron, the swing plane should be flatter at every stage throughout the downswing. The driver swing plane angle for this study was in the range reported by Williams and Sih (2002). Through the impact area, they reported a driver swing plane of $131.5 \pm 3.9^{\circ}$ which compares closely with the swing plane measured through impact for the swing of Golfer-Medium with Club-Regular $\left(130.1^{\circ}\right)$. Therefore, even though the golfer portion of the model was limited to 4 degrees of freedom, it was still able to represent a varying swing plane that is similar to that of actual golfers. Notice that the swing plane graph begins at $\mathrm{t}=0.06 \mathrm{~s}$ (Fig. 3.3.1.3.1). Coleman and Rankin started their plots of swing plane at the point where the clubhead reversed direction at the top of the backswing. Since all simulations in this thesis began with motion still existing in the backswing, Figure 3.3.1.3.1 was adjusted to meet the conditions defined by Coleman and Rankin. Note: the swing plane angle was also calculated according to the methods of Coleman and Rankin (2005). 


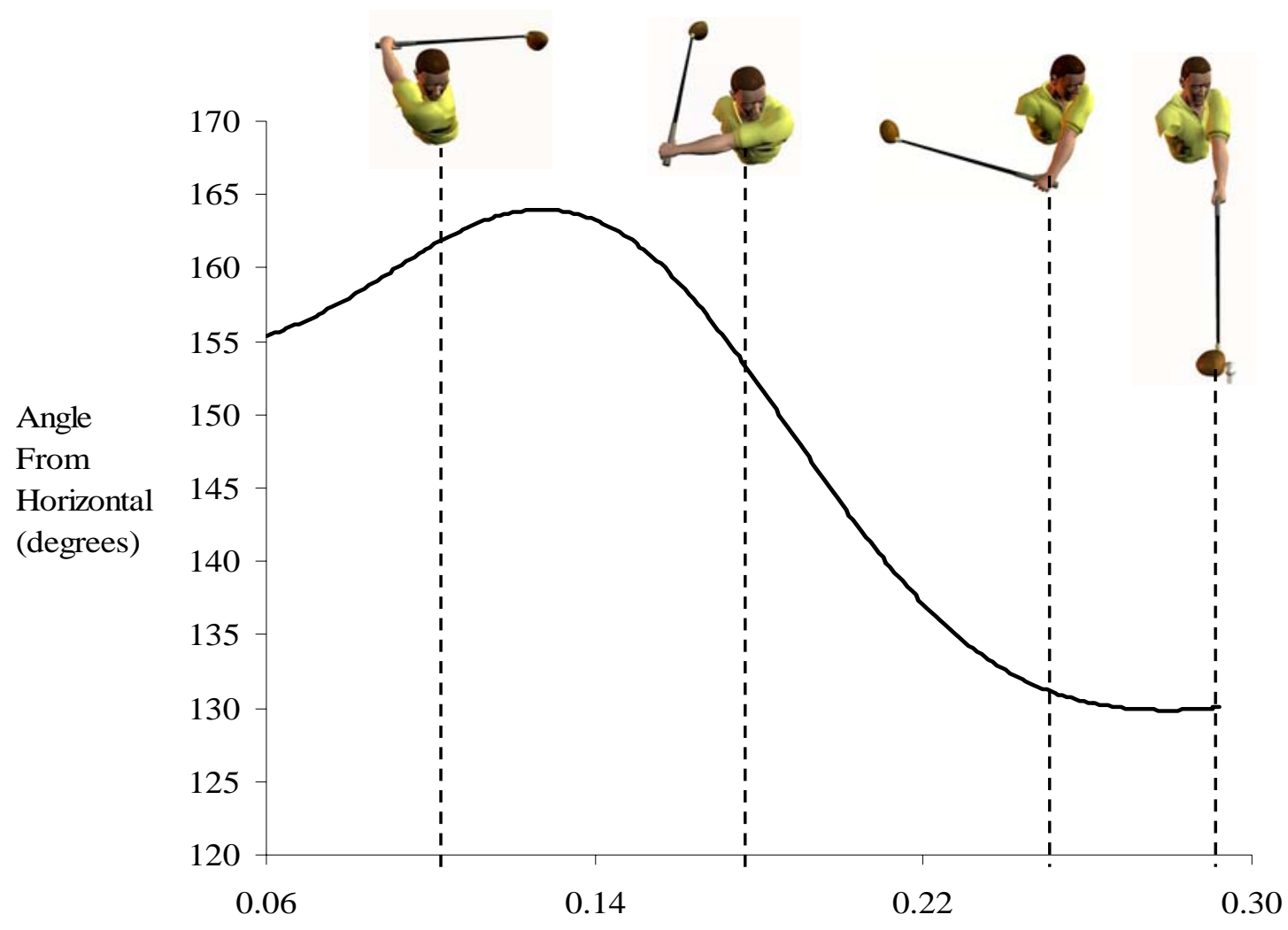

Time (s)

Figure 3.3.1.3.1 Angle of the swing plane relative to the horizontal for the optimized swing of Golfer-Medium with Club-Regular.

\subsubsection{Internal Validity}

Two separate procedures were conducted to measure the internal validity of the model. The first involved allowing the golfer model to rotate like a segmented pendulum about the center of the torso segment only under the influence of gravity. During the simulation, a function that was equal to the sum of the potential and kinetic energies of all segments in the model was calculated. The value of this function remained constant during the simulation which suggested that the equations of motion were formulated correctly. The second procedure involved the implementation of an integration checking function during all actual simulations used to generate results for 
this thesis. This integration checking function also remained at a constant value during all simulations which implies that the model was developed correctly in accordance with Newton's Laws of Motion. It also confirms that the level of error introduced by the numerical solving of the differential equations was not large enough to be a concern. 
4

DISCUSSION 
The purpose of this thesis was to further the understanding of the role of shaft stiffness in executing a golf drive. A thorough set of experiments was conducted to answer explicit questions related to this goal. Specifically, how does shaft stiffness affect clubhead speed and how does it alter clubhead orientation at impact. Additional experiments were performed to determine the underlying mechanisms responsible for the pattern of shaft deflection occurring during the downswing. For the first time, a 3D mathematical model of a golfer and club was developed and employed to answer these questions. This discussion will integrate the results from all experiments to address the hypotheses posed at the beginning of this thesis and to establish a comprehensive theory that explains the pattern of shaft deflection during the downswing.

\subsection{The Relationship Between Shaft Stiffness and Clubhead Speed}

Customizing the stiffness of a golf club shaft to perfectly suit a particular swing will not increase clubhead speed (and therefore ball speed) enough to have any meaningful effect on performance. This statement is confirmed by the clubhead speed results generated from the simulations (Table 3.1.2.1). No single shaft stiffness out performed the other two at any level of swing speed. At any given level of swing speed, the difference in clubhead speeds across levels of shaft stiffness did not exceed $0.1 \mathrm{~m} / \mathrm{s}$. An attempt was made to fine tune shaft stiffness even further by matching all possible levels of shaft stiffness with a single swing. The results indicated that regardless of swing speed, clubhead speed increased marginally as shaft flexibility decreased. 
Unfortunately, the marginal increases in clubhead speed were also accompanied by unacceptable configurations of the golfer-club models at impact.

Previous studies have shown that shaft flexibility can increase clubhead speed via the contribution from kick velocity (Butler \& Winfield, 1994; Miao et al., 1998). Even in this thesis, a kick velocity of $10.51 \mathrm{~m} / \mathrm{s}$ (Table 3.1.2.1) at impact was recorded for Golfer-Fast with Club-Regular suggesting that clubhead speed at impact would have been reduced by $10.51 \mathrm{~m} / \mathrm{s}$ if the shaft were perfectly rigid. Yet when Golfer-Fast was matched with Club-Rigid, clubhead speed was only reduced by $1.87 \mathrm{~m} / \mathrm{s}$ in comparison to Club-Regular. Also, kick velocities at impact for Golfer-Fast/Club-Flexible (10.51 $\mathrm{m} / \mathrm{s})$ and Golfer-Fast/Club-Stiff $(9.55 \mathrm{~m} / \mathrm{s})$ differed by approximately $1 \mathrm{~m} / \mathrm{s}$, yet both simulations resulted in the same clubhead speed of $52.94 \mathrm{~m} / \mathrm{s}$ at impact (Table 3.1.2.1). These findings show that kick-velocity is a misleading variable. An understanding of how kick velocity is produced is provided in the following paragraph and will explain why kick velocity does not simply add on to the clubhead speed generating capabilities of the golfer.

In an attempt to square the clubface for impact, tangential forces were applied at the grip end of the club just past the halfway point into the downswing. This resulted in the clubhead being deflected into a lagging position and the consequent storage of energy in the rotational springs joining the shaft segments. This storage of energy was accompanied by the rotational springs generating torques that tended to prevent deflection in the lag direction and support deflection in the lead direction. As impact approached, the tangential forces causing the lag deflection decreased which allowed the torque from the rotational springs to deflect the clubhead from its lagging position into a 
leading position. This could also be referred to as the release of strain energy and was facilitated by the action of radial force which was approaching its maximum value near impact. This process certainly increased clubhead speed relative to the most proximal club segment. Yet, it also served to simultaneously impede the absolute angular velocity of the most proximal club segment which was a detriment to clubhead speed. This happened because at the same time the restoring torque of the deformed rotational spring served to increase the angular velocity of the distal segment, an equal and opposite torque served to decrease the angular velocity of the proximal segment. However, the golfer model did have some ability to oppose the decrease in angular velocity of the most proximal club segment based on the properties of the muscle torque generators. This is evident when comparing the clubhead speeds attained with the rigid and non-rigid clubs (Table 3.2.1.1). Since clubhead speeds were greater with the nonrigid clubs, it shows that the shaft did have some ability to store and release energy during the swing. However, when comparing the non-rigid clubs, no particular level of shaft stiffness had a superior ability to increase clubhead speed, through the contribution of kick velocity, during the simulated swings (Table 3.1.2.1).

A golfer does not have the ability to produce constant levels of acceleration during the downswing which has important implications when considering the potential contribution from kick velocity. Researchers have developed golfer models which employed fixed levels of acceleration during the downswing in an attempt study shaft flexibility (Jorgensen, 1994; Miao et al., 1998). This is not a reasonable assumption since the golfer model must be able to interact with the dynamic properties of the club. If fixed functions of acceleration were used in this thesis in place of the muscle torque 
generators, then the importance of shaft flexibility in contributing to clubhead speed would have been greatly over estimated. If the angular acceleration of the most proximal club segment was predetermined, then the torque from the rotational springs would only serve to increase clubhead speed and not decrease the angular velocity of the most proximal club segment. This would result in spuriously large clubhead speeds. It is also likely that golfer robots may suffer from this same limitation; namely, the inability to dynamically interact with the properties of a golf club in the same way as a live golfer.

Currently, no researcher has presented conclusive results showing that different shaft flexibilities result in measurably higher clubhead speeds at impact and therefore, subsequently higher ball speeds. Miao et al. (1998) presented data which suggested that certain levels of shaft stiffness resulted in higher clubhead speeds for a particular golfer. However, as previously indicated in this thesis, it appears as though the error levels associated with the data presented by Miao et al. were greater then the differences that were measured in clubhead speeds across levels of shaft stiffness.

Based on the previous arguments it should not be inferred that certain levels of shaft stiffness cannot outperform others for a particular swing when considering increased driving yardage. Rather it should be understood that if driving distance was found to be meaningfully different across levels of shaft stiffness, then that increased driving yardage would be a result of factors other then differences in ball speed. These other factors, namely, ball launch angle and ball spin rate are influenced by clubhead orientation at impact which is in turn determined by shaft deflection. 


\subsection{The Relationship Between Shaft Stiffness and Clubhead Orientation}

Aside from golf ball speed which is primarily a function of clubhead speed, ball launch angle and spin rate are the only other two factors under the control of the golfer that can affect driving distance. For a perfectly impacted golf ball, no side spin is imparted to the ball and the flight of the ball does not deviate from a path directly above the target line. Under these conditions, launch angle refers to the angle between the velocity vector of the ball and the horizontal immediately after impact, while spin rate refers to the amount of angular velocity (back spin) imparted to the ball. With respect to the execution of a golf drive, spin rate is primarily a function of clubhead loft (including dynamic loft), while launch angle is a function of both clubhead loft and clubhead path (Winfield \& Tan, 1994). If a golfer has a consistent swing, then the path of the clubhead relative to the ball will not change from swing to swing. Therefore, by adjusting the amount of clubhead loft relative to the ball, an optimal ball launch angle and ball spin rate can be found for achieving maximum driving distance. Results from the computer simulations demonstrated that clubhead loft can change by as much as $0.65^{\circ}$ depending on shaft stiffness for a golfer with a swing speed of approximately $45 \mathrm{~m} / \mathrm{s}$ (101 mph) (Table 3.1.2.1). For example, the Golfer-Medium $\backslash$ Club-Stiff simulation resulted in $4.82^{\circ}$ of dynamic loft at impact, while the Golfer-Medium $\backslash$ Club-Flexible simulation resulted in $5.47^{\circ}$ of dynamic loft. The results from the optimization study conducted by Winfield and Tan suggest that a loft change of this magnitude may be enough to have a meaningful influence on driving distance. However a golfer must swing very consistently to take advantage of these changes. Also, larger differences in clubhead 
loft could be expected when comparing the non-optimized swings of many amateur golfers.

It should also be pointed out that the loft increases brought about as a result of shaft flexibility could also be made simply by changing the static loft of the club. For example, clubhead loft dynamically increased by $0.65^{\circ}$ when Golfer-Regular was matched with Club-Flexible $\left(5.47^{\circ}\right)$ in comparison to the Golfer-RegularlClub-Stiff $\left(4.82^{\circ}\right)$ combination (Table 3.1.2.1). These two simulations would theoretically produce different ball flight conditions because of the discrepancy in clubhead loft at impact. If the face angle of Club-Stiff was geometrically changed to increase the static loft of the club by $0.65^{\circ}$, both conditions would then produce the same theoretical ball flight. This would be the case because the clubhead paths between conditions were already not meaningful different (not previously reported), and the clubhead speeds were already very similar $(44.96 \mathrm{~m} / \mathrm{s}$ vs. $45.04 \mathrm{~m} / \mathrm{s})$. The effect of statically changing the loft of clubface was confirmed using the model. Statically increasing the loft from $10^{\circ}$ to $10.65^{\circ}$ resulted in the loft at impact increasing by $0.6496^{\circ}$. Physically altering the loft of the clubface will not change the position of the center of mass of the clubhead and therefore, will have no influence on shaft deflections during the swing.

In summary, clubhead path, loft and speed influence ball flight. As a variable, only shaft stiffness was found to have a meaningful effect on clubhead loft which can also be changed by altering the physical geometry of the club. Therefore, it seems that shaft stiffness is not a necessary variable to consider when fitting a driver to a golfer's swing. 
However, there are two other factors to consider before defining the role of shaft stiffness. First, perhaps solely manufacturing a more complete spectrum of clubhead geometries with a single shaft stiffness is not practical. It may be more economically feasible for club manufacturers to provide a range of both clubhead geometries and shaft stiffness that produce the desired impact conditions. Second, the influence of feel cannot be easily dismissed. Certainly, the feel of the driver is linked to the golfer's confidence in executing the shot which will inevitably weigh heavy on the final outcome of the swing.

\subsection{The Mechanisms Behind Shaft Bending}

Both radial and tangential forces, relative to the circular path traced by the longitudinal axis of the shaft, played important roles in shaft deflection during the downswing and in the resulting clubhead orientation at impact. The possible mechanisms behind shaft bending were previously discussed in the introduction. It was argued that using a conceptual frame work that described forces that were either radial or tangential to the longitudinal axis of the shaft would help understand the cause of shaft deflection during the downswing. The same conceptual frame work will be used in this discussion.

The largest magnitude of shaft deflection during the downswing was in the toeup direction. For the computer simulation swings of Golfer-Medium, the maximum toeup deflections were approximately $10 \mathrm{~cm}$ in magnitude and occurred during the initial part of the downswing. Tangential forces acting along the $x$ axis were the primary cause

of these deflections. Contrary to popular belief, this initial deflection had no impact on 
clubhead speed and little influence on the final orientation of the clubface at impact. Initial bending in the toe-up direction may superficially appear to be storing energy which could later be released to increase clubhead speed. However, due to the $90^{\circ}$ rotation of the club about the lead arm during the final stage of the downswing, none of the energy stored in the initial part of the swing could ever be returned to the clubhead along the intended direction of ball flight. Further, any residual effects of this deflection present at impact will only have a small effect on the dynamic loft of the club. Based on the results of this thesis, it would appear that the initial bending in the toe-up direction would only serve to increase the variability in a golf swing. This information supports the view of those golf instructors that advocate a 'smooth' transition into the downswing, as opposed to a rushed transition that would lead to larger shaft deflections early in the downswing.

The next important period of shaft deflection occurred in the lag direction over the final half of the downswing. For the computer simulation swings of GolferMedium, the maximum lag deflections were approximately $3.5 \mathrm{~cm}$ in magnitude. Tangential forces, acting along the $y$ axis were the primary cause of deflections in the lag direction. Shaft deflection in this direction resulted in the storage of strain energy that had the potential to be released near impact and result in a faster clubhead speed. This period of shaft deflection cannot be predicted from a 2D model and is essentially why a 3D simulation was needed to sufficiently model the downswing.

The final phase of shaft deflection was the most important since it described clubhead orientation at impact. It was also the most complex because both tangential and radial forces played meaningful roles, and because it occurred over a relatively short 
time period $(0.03 \mathrm{~s})$. Over the final few hundredths of a second of the downswing, the clubhead rapidly moved from its maximum lagging position into its maximum leading position at impact. For the computer simulation swings of Golfer-Medium, the lead deflections at impact were approximately 6.0 to $6.5 \mathrm{~cm}$ in magnitude. The complete removal of radial force during the downswing only reduced lead deflection to $4.72 \mathrm{~cm}$ (Fig. 3.2.3.1). Therefore, when acting in isolation, the tangential forces were responsible for a considerable portion of the lead deflection at impact. The complete isolation of radial force demonstrated that while acting alone, radial force could only result in $1.22 \mathrm{~cm}$ of lead deflection at impact (Fig. 3.2.3.2). Summing the lead deflection that occurred from the isolation of the two sets of forces results in a lead deflection of $5.94 \mathrm{~cm}$, which is $0.31 \mathrm{~cm}$ less than when radial and tangential forces acted simultaneously. This finding suggests that there is an interaction effect between the force components in terms of lead deflection.

Toe-down deflection at impact was also affected by both radial and tangential force components. Both radial and tangential force components contributed approximately equally to the magnitude of toe-down deflection at impact. When acting in isolation, radial force was shown to deflect Club-Regular by $-1.33 \mathrm{~cm}$ in the toe-down direction. For the optimized swing of Golfer-Medium with Club-Regular, toe-down deflection was $-2.26 \mathrm{~cm}$ at impact; therefore, just over half of that deflection was the direct result of radial force action. The additional deflection was the result of the club recoiling from its toe-up deflected position earlier in the downswing. 


\subsection{The Role of Radial Force}

Several researchers claim that radial force is the dominant factor producing shaft deflection at impact (Mather \& Cooper, 1994; Butler \& Winfield, 1994, Horwood, 1994; Milne \& Davis, 1992; Mather \& Jowett, 1998). The results from section 3.2.3 demonstrated that when radial force acted in isolation, shaft deflection in either the toedown or lead direction did not exceed $1.33 \mathrm{~cm}$ in magnitude. Yet, several authors (Mather \& Cooper, 1994; Butler \& Winfield, 1994, Horwood, 1994), as well as, the results from this thesis have demonstrated that shaft deflection at impact can exceed 4 $\mathrm{cm}$ in both directions. A possible explanation of these ambiguous findings is that the radial force generated by the model used in this thesis was not large enough to produce the previously reported magnitudes of shaft deflection.

However, the peak magnitude of radial force (456 N) measured during the optimized swing $(45 \mathrm{~m} / \mathrm{s})$ of Golfer-Medium with Club-Regular was within the range presented in the literature. Williams (1967) deduced that Bobby Jones generated a clubhead speed of approximately $50 \mathrm{~m} / \mathrm{s}$ and applied a radial force of $476 \mathrm{~N}$ to his driver at impact. Vaughn (1981) (360 N) and Neal and Wilson (1985) (315 N) reported reduced values for peak radial force during the downswing. However, these researchers generated their results from 3D inverse dynamic analyses of live golfers which bring into question how much their smoothing techniques reduced peak values. Miura (2001) predicted a radial force of $414 \mathrm{~N}$ from his $2 \mathrm{D}$ model which generated a clubhead speed of $46.8 \mathrm{~m} / \mathrm{s}$. Mather and Jowett (1998), state that radial force approaches $500 \mathrm{~N}$ for clubhead speeds of $45 \mathrm{~m} / \mathrm{s}$. Considering these results, it appears as though $456 \mathrm{~N}$ is a reasonable prediction of radial force. 
Another possible explanation is that the simulated clubs used in this thesis were too stiff to permit radial force to generate the previously reported magnitudes of shaft deflection. This explanation seems doubtful when the peak magnitudes of shaft deflection in the toe-up direction, and lag direction are compared to previous research. Butler and Winfield (1994) reported peak toe-up deflections (15 cm) and peak lag deflections $(7 \mathrm{~cm})$, that were greater than the respective measurements from the simulation results in this thesis $(10.69 \mathrm{~cm}$ and $4.79 \mathrm{~cm})$. Milne and Davis (1992) also reported peak toe-up deflections exceeding $10 \mathrm{~cm}$ in magnitude.

In summary, radial force plays an important role in facilitating the toe-down and lead-deflections recorded in all 'golf' swings made with a driver. However, the recoil of the shaft from its previously toe-up and lag deflected position, due to tangential forces, plays at least an equally important role in determining the final position and orientation of the clubhead.

\subsection{Limitations of the Model}

As all models do, the mathematical model used in this thesis did not completely represent every aspect of the target system. The purpose of this section is to address the limitations of the mathematical model.

The role of the golfer portion of the mathematical model was to apply a realistic temporal pattern of forces and torques to the grip end of the club model. The two segment golfer representation employed to fulfil this role was able to reproduce most of the motion of a live golfer. The fact that the torso segment was constrained to rotate around a fixed axis meant that the linear motion of the trunk, that might result from the leg action of a live golfer, was not represented in the model. The lack of linear motion 
in the trunk was not regarded as a major limitation since the golf swing is predominantly rotational. However, due to the lack of direct leg musculature contribution, it was likely that the outputs from the muscle torque generators were slightly greater than that of a live golfer with a similar clubhead speed. Related to the absence of legs from the model is the absence of hip rotation. It is generally agreed upon that the first movement into the downswing is hip rotation. In the popular golfing literature, a large difference in angular rotation between the hips and shoulders early in the downswing is often cited as a good indicator of a properly executed swing. The absence of a pelvic girdle in the model simply means the absence of another segment which could contribute to the overall motion. This would also likely lead to slightly greater muscle torque outputs. The golfer model used in this thesis also incorporated any contribution from a trail arm into the wrist adductor of the lead arm. It is possible that this representation may have influenced the clubhead speed results from section 3.1.. It was determined in section 3.1 that, for a given golfer, altering the stiffness of a golf shaft would have no meaningful effect on clubhead speed. This was the result of the golfer model dynamically interacting with the torques produced by the spring-damper elements of the shaft. The torques from the spring-damper elements served to increase kick velocity while simultaneously reducing the angular velocity of the most proximal club segment. It is possible that incorporating a trail arm into the model may have offset the effect of the bending moments in the shaft on the most proximal club segment's angular velocity. However, I feel that this is not likely for two reasons. First, the torque generating capabilities of the wrist adductor were increased to represent both the wrist adductor and trail arm contributions to the downswing. Second, the torques responsible for increases 
in kick velocity occurred very late in the downswing. This would place the trail arm in a compromised position from which to apply forces to the club.

Although, the muscle representation used in the golfer model is more encompassing than those used in previous golfer models, it does not match the complexity of similar models used to simulate jumping and walking. Essentially the potential limitations of the muscle models are that they represent overall joint moments and not individual muscle forces, neural inputs were not incorporated, and the lengthtension property of muscle was not modelled. The important question is how do these simplifications affect the pattern of forces and torques experienced by the golf club?

Employing individual muscle models is important if the purpose of the research is to understand the role of individual muscles to the overall movement pattern. This is often the case in studies that model jumping. However, if the purpose is merely to generate an accurate representation of the real system's kinematic pattern, then torque generators are sufficient. Therefore, the lack of individual muscle force representation has little bearing on the results generated to investigate the hypotheses posed in this thesis. From the viewpoint of the golf club, it makes no difference if four separate muscle force generators add energy into the arm segment, or if that role is fulfilled by a single muscle torque generator. The resulting motion of the golfer model and consequently the kinetics imposed on the club remain the same.

Incorporating neural stimulation into the model allows graded levels of "muscular effort” to be achieved during the simulation. This is obviously important in models such as walking in which muscular effort is always supplied to the skeletal system at sub- maximal levels. However, one goal for a golf drive is to displace the golf 
ball as far off the tee as possible. This requires maximal clubhead speed at impact which in turn necessitates a near maximum level of muscular effort during the downswing given the optimal technique. Based on this argument, it is doubtful that integrating neural stimulation would provide a more accurate representation of the overall joint torque profiles generated during a golf drive for maximum distance. The muscle torque generators were also able to instantaneously deactivate. Although actual muscle cannot instantaneously deactivate, this was thought to have little influence on the pattern of shaft deflection.

The length-tension property of skeletal muscle certainly plays an important role in modulating the level force capable from an activated muscle. However, according to Caldwell (1995), the length-tension property is not as important for determining muscle force output as either the activation level, or force-velocity characteristics of muscle. It is also likely that during the execution of a golf drive, the sarcomeres of the active muscles are not moved through the extreme positions on the length-tension curve. Based on this information and the difficulty of assigning a length-tension relationship to a torque generator representing several muscles it was decided to accept this as a limitation in the model.

Accurately measuring and consequently modelling the damping brought about by the connection of club with the soft tissue in the hands of the golfer is a difficult task (Brylawski, 1994; Mather \& Cooper, 1994; Milne \& Davis, 1992). In the end, this was not so much a limitation as it was a parameter that had to be estimated. Essentially, the only confirmation that the level of damping chosen was adequate was the agreement of 
the simulation results with the live golfer testing results presented in this thesis and in past research.

An important consideration in this thesis was how to provide the simulated club with the ability to deflect in the necessary directions. It was decided to divide the shaft into segments connected by rotational spring-damper elements. Perhaps a finite element model of a club would have proven to be more externally valid. However, due to software and programming limitations that wouldn't permit the amalgamation of a finite element model with a 3D musculoskeletal model, a finite element approach was not feasible. Undoubtedly, a finite element representation would have provided a better overall shape of a continuous shaft deflection along the length of the shaft. However, it was felt that a finite element model would have yielded the same results as the rotational spring-damper method with regards to kick-velocity and the magnitudes of deflection. The simulated clubs were divided into only four segments due to the size of the equations of motion that resulted. Essentially, the motion equations became too large to be incorporated into the forward dynamics program if more than four club segments were included with the two golfer segments.

\subsection{Limitations of the Live Golfer Testing}

The high velocity of the shaft through the impact area, the 3D nature of the swing, and the relatively small magnitudes of shaft deflection all make the accurate recording of the pattern of bending during the downswing difficult. It was decided that high speed video would be used to capture the shaft bending data. This was chosen over a strain gage collection method used by other researchers simply because of the visual confirmation of results. The limitation of a single camera required the implementation 
of separate set-ups to measure shaft bending in both the toe-down/toe-up and lead/lag directions. This was not ideal since deflection data for both bending directions could not be obtained from a single swing. The set-up from Perspective 2 with the camera behind the golfer would also not permit the determination of clubhead speed for those trials. This meant that a relationship between toe-down deflection and clubhead speed could not be analyzed.

\subsection{Conclusions}

The following conclusions address the purpose and hypotheses stated in the introduction to this thesis and are based on the results that emerged from the experiments described in the methods section.

(1) A six-segment, 3D mathematical model of a golfer driven by muscle torque generators can be successfully used to model the behaviour of a golf shaft during the downswing. This supports hypothesis 1.

(2) Tangential forces applied by the golfer to the club handle are the major contributors to the shaft deflections that occur during the first $90 \%$ of the downswing. Tangential forces play at least an equally important role as radial force in determining the final deflected position of the club at impact. This supports hypothesis 2 . 
(3) Radial force serves to increase the magnitudes of the toe-down and lead deflections present at impact during optimal swings with a driver.

(4) The simulation results showed that the flexibility of a golf shaft has no influence on clubhead speed for golfers of different swing speed capabilities. The supports hypothesis 3.

(5) All optimal golf swings with a driver will result in the shaft being deflected in the toe-down and lead directions at impact. For golf swings with a similar pattern of force and torque application to the club, as swing speed increases so does the magnitude of shaft deflection at impact. This supports hypothesis 4 .

(6) For an optimal golf swing, as shaft stiffness decreases the magnitude of shaft deflection at impact increases. This supports hypothesis 4 .

(7) Altering the position of the center of mass of the clubhead affects clubhead orientation at impact by an amount proportional to the size of the change in position. The change in clubhead orientation associated with altering the center of mass of the clubhead is a result of the action of radial force. This supports hypothesis 5 . 


\subsection{Future Directions}

I believe that the research conducted in completing this thesis sufficiently answered the problem posed at the outset. However, provided with additional and improved resources, enhancements to the methodology and thus quality of the results could have been made. The current model also permits the consideration of other questions related to the dynamics of the golf swing.

As mentioned in the review of forward dynamics, the external validity of the model improves as the number of segments incorporated into the model increases. This assumes that the segments added contribute to the overall motion of the system that is being modeled. In the future, attempts should be made to incorporate the action of the legs and hips. The legs do provide some linear motion to the system, and the addition of

a pelvic segment would take some of the torque generating responsibility away from the other muscle torque generators, thus resulting in more realistic overall torque profiles.

The incorporation of a trail arm would improve the representation of the golfer model as well. I believe that elbow extension of the trail arm late in the downswing is critical to the proper execution of a well timed golf swing. In the current model, the contribution from the trail arm is essentially represented by the wrist adduction torque generator. This results in muscle torque capabilities that likely exceed the ability of the wrist adductors. An interesting phenomenon that involves the force-velocity properties of muscle also comes to light if the trail arm were to be included. The relative angular velocity of the club near impact is very high. In fact, the club is likely rotating too quickly for the wrist adductors or external rotators of the lead arm to apply any force due to the force-velocity constraints of skeletal muscle. However, assuming that the 
elbow extensors of the trail arm are the kinetic cause of any torque applied to the club late in the downswing, then the force-velocity constraint is no longer a major issue. The physical geometry of the trail arm/golf club connection allows the elbow to move through a relatively small range of motion in comparison to the angular displacement of the club.

The notion of replacing the torque generators with individual muscle force generators could also be attempted. Although it is not foreseeable that this would impact the findings from this thesis, such a muscular representation would shed some light on contribution from individual muscles during the downswing.

Given the current popularity of finite element modeling and the increasing availability of software and programming information on finite element modeling, it is the most evident aspect that could be improved upon. Although I was not able to find a satisfactory method of amalgamating a 3D musculoskeletal model with a finite element model of a golf club, the potential still exists. As an initial step in this direction, perhaps the force and torque profiles applied to the club by the golfer model could serve as input into a finite element model of a club. However, this would not be completely satisfactory, as the golfer model would not be able to dynamically interact with the club during the swing. A finite element model of the impact of the club and ball would also be the next logical step in quantifying the effects of shaft stiffness on the actual ball flight characteristics.

Although it was decided that mathematical modeling was the best way to approach the problem posed in this thesis, improved experimental procedures would be invaluable in supporting the conclusions. To experimentally determine if shaft stiffness 
could meaningful influence clubhead speed, several conditions would have to be met. Professional golfers with highly repeatable swings would have to be employed. As in this study, golf clubs identical in all aspects with the exception of shaft stiffness would need to be incorporated. Perhaps most import, very accurate measurements of golfer motion and clubhead speed would have to be recorded over many trials. The measurements would have to be extremely accurate $( \pm 2 \mathrm{~mm})$. The data collection system would also need a very high sampling rate (at least $500 \mathrm{~Hz}$ ), and be able to track the complicated $3 \mathrm{D}$ motion of the club in a relatively large volume. With this experimental set-up, a researcher should be able to ascertain whether clubhead speed can be influenced by shaft stiffness.

Although not related to the purpose, a very interesting finding occurred in the development of the 3D golfer-club model used in this thesis. This finding, which I will explain below, not only provides an important direction for future investigation, but also serves as an excellent anecdote on the advantages and even necessity of forward dynamic models.

The kinematics of the golf swing are relatively complex. The kinetics that produced the kinematics are, in my opinion, inherently more complex. In considering the optimal execution of a skill requiring musculoskeletal motion, the researcher or individual instructing the skill is interested in what the performer must do to execute the skill. More explicitly, what contributions from the muscles of the performer are necessary to attain the observed optimal kinematics? This is not necessarily obvious from the measured kinematics. For example, an individual with a below the knee prosthesis is still able to perform knee flexion and extension movements during walking 
without any contribution from muscles crossing the knee. These motions are caused by the joint reaction forces at the knee and the resulting momentum. Similar principles are at work in the golf swing, but are much more difficult to visualize than the prosthetic example just described, due to the high velocity and 3D nature of the swing. A forward dynamics model permits the deciphering of what motions result from the "passive forces” occurring during the golf swing, and those motions which are a direct result of muscular action. This information is essential to teaching and the correct execution of a golf swing. The following describes such a finding that emerged from the development of the golfer model used in this thesis.

The muscle torque generators present in the golfer model can directly generate four basic movements. Three of those movements: torso rotation, shoulder abduction, and wrist adduction can move the golf club through the downswing, to the point of impact, but cannot directly cause the clubface to square up for impact (Fig. 4.7.1 A, B, C, D and E). The fourth movement, rotation of the lead arm about its longitudinal axis, would appear on first inspection to provide the only mechanism to actively square up the clubface for impact (Fig. 4.7.1 F). 

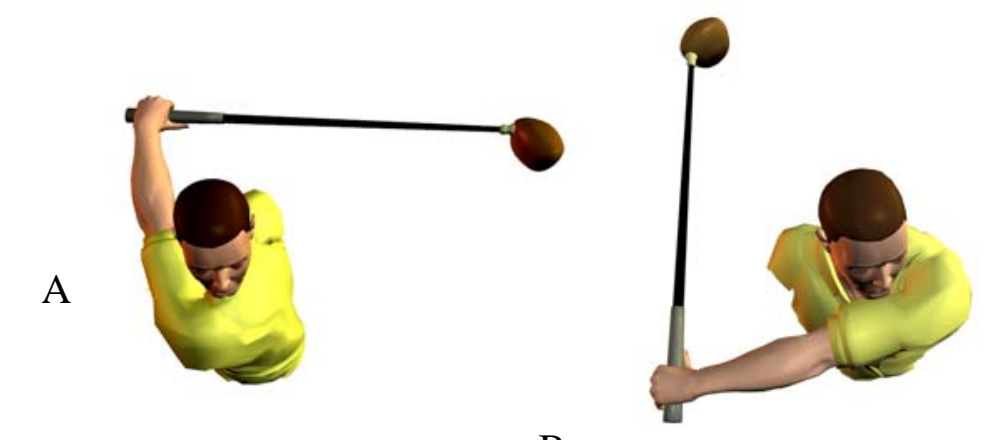

B
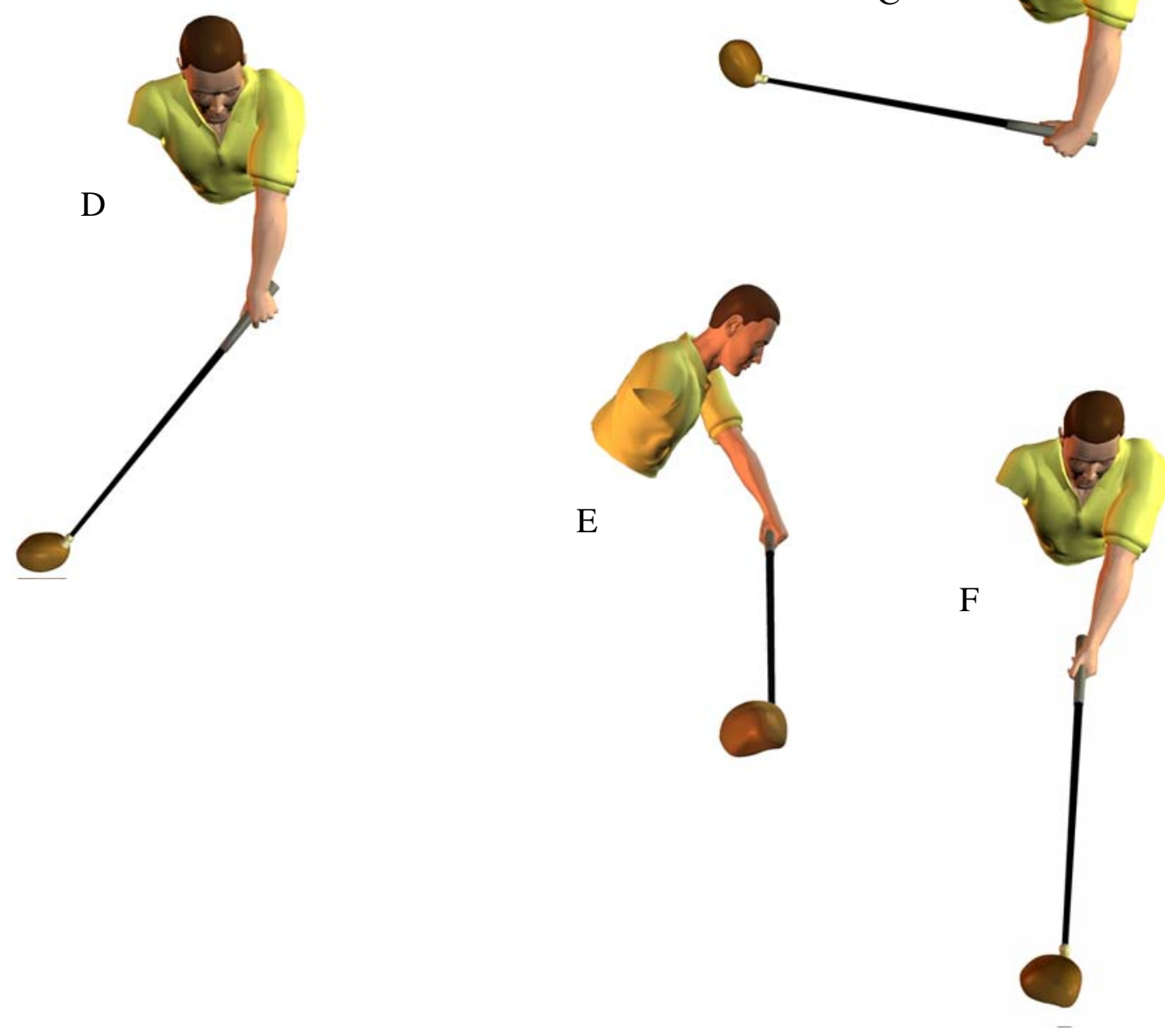

$\mathrm{C}$

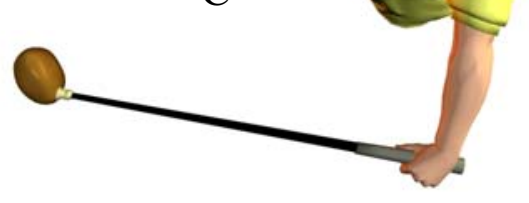

Figure 4.7.1 A. Position at start of downswing. B. Torso rotation. C. Shoulder abduction. D. Wrist adduction. E. Wrist adduction from behind. F. Rotation of the lead arm about its longitudinal axis. Note: the above sequence is not from a simulated swing, but rather was generated solely to demonstrate specific movements. 
However, in the development of the golfer model it was determined that, during the execution of a golf drive, a torque acting about the longitudinal axis of the lead arm was not necessary to square to the clubface for impact. Obviously, rotation about the longitudinal axis of the lead arm was still required, but this rotation did not occur due to a torque produced by a muscle torque generator. Rather, joint reaction forces and gravity were the kinetic factors behind the squaring of the clubhead for impact. Consider a golfer model that has muscle torque generators that would produce the movements described in the Figure 4.7.1 with the exception of a torque generator that would produce rotation about the longitudinal axis of the lead arm. The arm is free to rotate about its longitudinal axis, but no torque generator acts about its longitudinal axis of the lead arm. Since gravity acts at an angle to the plane in which shoulder abduction occurs, it will tend to cause rotation about the longitudinal axis of the lead arm due to the position of the club's center of mass (Fig. 4.7.2A). This gravitational force results in the club 'falling' below the arm abduction plane (Fig. 4.7.2B). Due to this position inside the plane, a force acting at the grip end of the club, and within the arm abduction plane, produces a torque on the club about the longitudinal axis of the lead arm. This torque will act until the center of mass of the club moves within the arm abduction plane. As the center of mass moves towards the arm abduction plane, the angular impulse generated by the torque creates angular momentum about the longitudinal axis of the lead arm. It is this angular momentum about the longitudinal axis of the lead arm that caused the clubhead to square for impact in absence of muscle torque acting about the longitudinal axis of the lead arm. Certainly, a properly timed muscle torque acting 
about the longitudinal axis of the lead arm can increase clubhead speed, but it is not necessary to attain the desired impact position with the club.

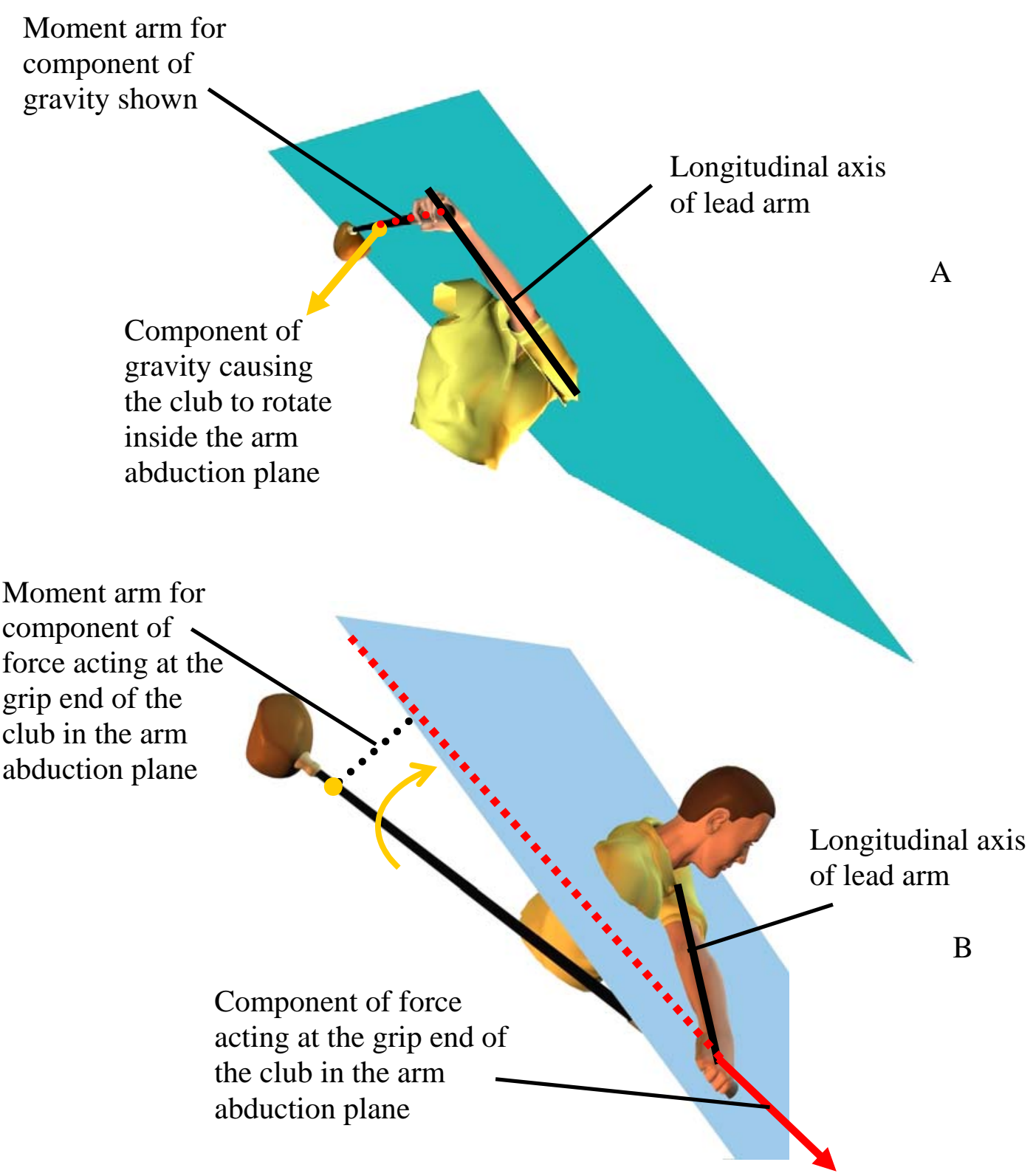

Figure 4.7.2 A. At the start of the downswing, gravity tends to pull the club below the plane formed by arm abduction. B. Midway through the downswing, a component of force acting within the arm abduction plane produces an angular impulse on the club about the longitudinal axis of the lead arm. 
Of equal interest is the converse scenario. Assume now that the golfer can exert a muscle torque about the longitudinal axis of the lead arm. If that torque is exerted too early in the downswing (the golfer attempts to square the club for impact prematurely), then the club moves into a position above the arm abduction plane (Fig. 4.7.3). From this position, the force acting on the grip end of the club will produce a torque about the longitudinal axis of the lead arm that will tend to bring the club back down towards the arm abduction plane. This torque will act until the center of mass of the club moves within the arm abduction plane. As the center of mass moves towards the arm abduction plane, the angular impulse generated by the torque creates angular momentum about the longitudinal axis of the lead arm. Unfortunately for the golfer, the angular momentum generated in this scenario tends to inhibit the squaring of the clubface for impact. This results in an open clubface at impact, and the inevitable slicing action of the golf ball trajectory. This phenomenon only becomes clear through the implementation of a forward dynamics model. 


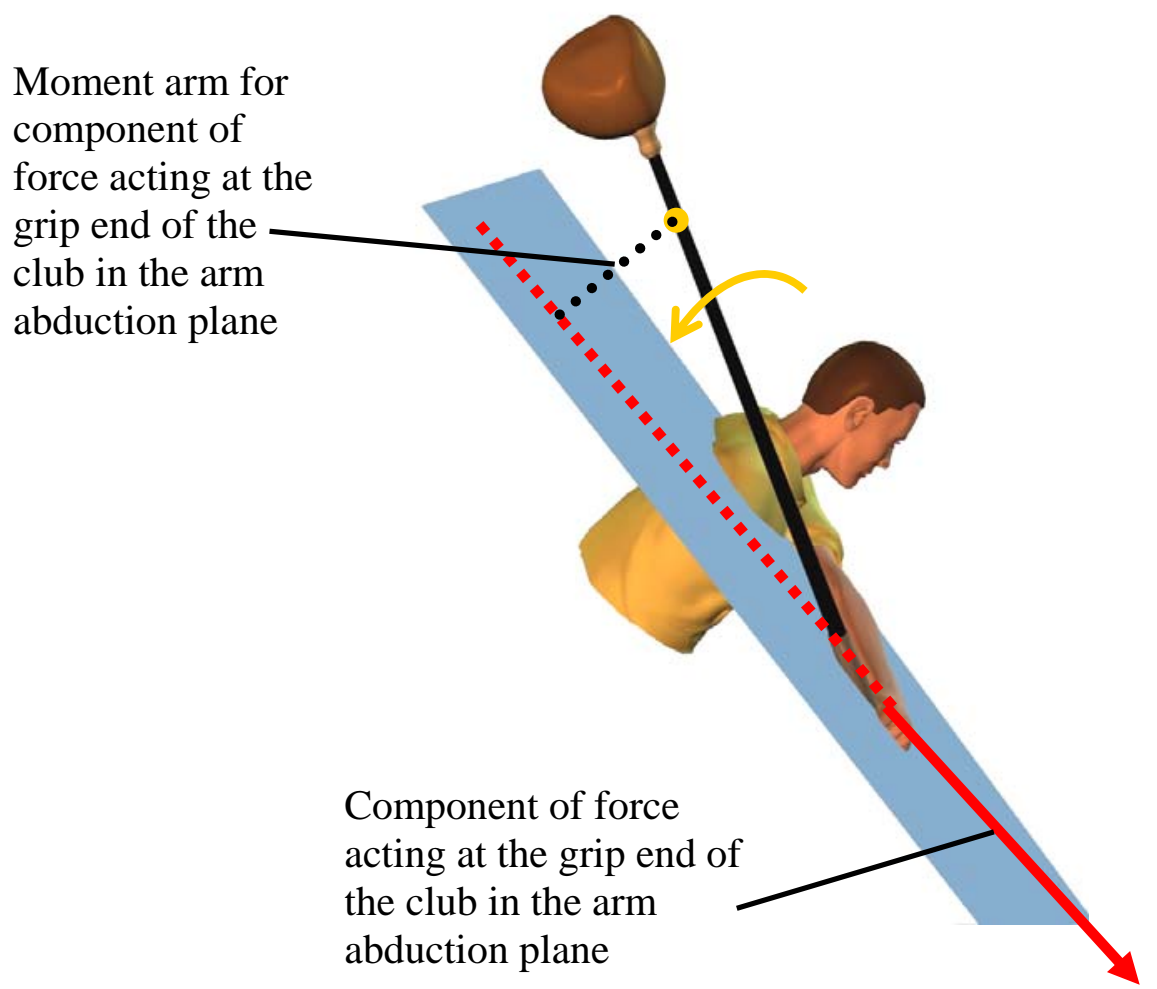

Figure 4.7.3 If the club is above the arm abduction plane midway through the downswing, a component of force acting within the arm abduction plane produces an angular impulse on the club about the longitudinal axis of the lead arm which tends to rotate the club back down into the plane. 


\section{REFERENCES}

Allard, P., Stokes, I. A. F., \& Blanchi, J. P. (1995). Three-dimensional analysis of human movement. Champaign, IL: Human Kinetics.

Altman D. G., \& Bland, J. M. (1983). Measurement in medicine: the analysis of method comparison studies. Statistician, 32, 307-317.

Andrews, J. G. (1995). Euler’s and Lagrange’s Equations for linked rigid-body models of three dimensional human motion. Chapter 8 in Three-dimensional analysis of human movement. Allard, P., Stokes, I. A. F., \& Blanchi, J. P. (Eds). Champaign, IL: Human Kinetics.

Atkinson, G., \& Nevill, A. M. (1998). Statistical methods for assessing measurement error (reliability) in variables relevant to sports medicine. Sports Medicine, 26, 217-238.

Brylawski, A. M. (1994). An investigation of three dimensional deformation of a golf club. In M. R. Farrally, \& A. J. Cochran (Ed.), Science and Golf II: Proceedings of the World Scientific Congress of Golf (pp. 265-270). United Kingdom: E \& FN Spon.

Butler, J. H., \& Winfield, D. C. (1994). The dynamic performance of the golf shaft during the downswing. In M. R. Farrally, \& A. J. Cochran (Ed.), Science and Golf 
II: Proceedings of the World Scientific Congress of Golf (pp. 259-264). United Kingdom: E \& FN Spon.

Caldwell, G. (1995). Tendon elasticity and relative length: effects on Hill twocomponent muscle model. Journal of Applied Biomechanics, 11, 1-24.

Cavanga, G. A., Citterio, G. (1974). Effect of stretching on the elastic characteristics and the contractile component of frog striated muscle. Journal of Physiology, 239, 1-14.

Cochran, A. J., \& Stobbs, J. (1968). The search for the perfect swing. London: Morrison \& Gibb Ltd.

Coleman, S. G. S., \& Rankin, A.J. (2005). A three-dimensional examination of the planar nature of the golf swing. Journal of Sports Sciences, 23, 227-234.

Cooper, M. A. J., \& Mather, J. S. B. (1994). Categorization of golf swings. In M. R. Farrally, \& A. J. Cochran (Ed.), Science and Golf II: Proceedings of the World Scientific Congress of Golf (pp. 65-70). United Kingdom: E \& FN Spon.

De Leva, P. (1996). Adjustments to Zatsiorsky-Seluyanov's segment inertia parameters. Journal of Biomechanics, 29, 1223-1230. 
Dudel, J. (1978). In R. Schmidt (Ed.), Fundamentals of Neurophysiology $2^{\text {nd }}$ Edition. New York: Springer-Verlag.

Edman, K. A., Elzinga, G., \& Noble, M. I. (1978). Enhancement of mechanical performance by stretch during titanic contraction of vertebrate skeletal muscle fibres. Journal of Physiology, 281, 139-155.

Fox, L. (1962). Numerical solutions of ordinary and partial differential equations. (pp. 24-25). Palo Alto: Addison-Wesley.

Hanavan, E. P. (1964). A mathematical model of the human body. Report no. AMRL-TR-64-102, AD-608-463. Ohio: Aerospace Medical Research Laboratories, Wright-Patterson Air Force Base.

Hatze, E. (1980). A mathematical model for the computational determination of parameter values of anthropometric segments. Journal of Biomechanics, 13, 833-843.

Hill, A. V. (1938). The heat of shortening and the dynamic constants of muscle. Proceedings of the Royal Society, 126B, 136-195.

Hill, A. V. (1970). First and Last Experiments in Muscle Mechanics. London: Cambridge University Press. 
Hogarth, K. L. (2002). An analysis of twisting dives. Ph.D. Thesis, The University of Queensland, Brisbane, Queensland, Australia.

Horwood, G. P. (1994). Golf shafts - a technical perspective. In M. R. Farrally, \& A. J. Cochran (Ed.), $\underline{\text { Science and Golf II: Proceedings of the World Scientific }}$ Congress of Golf (pp. 247-258). United Kingdom: E \& FN Spon.

Houk, J. C. (1963). A mathematical model of the stretch reflex in human muscle systems. M.S. Thesis, M.I.T., Cambridge MA.

Huxley, A. F. (1974). Muscular contraction. Journal of Physiology, 243, 1-43.

Jorgensen, T. P. (1994). The physics of golf. USA: AIP Press.

Jorgensen, T. P. (1970). On the dynamics of the swing of a golf club. American Journal of Physics, 38, 644-651.

Kirsch, B. G. (1998). The Americanization of golf: 1888-1914. In M. R. Farrally, \& A. J. Cochran (Ed.), Science and Golf III: Proceedings of the World

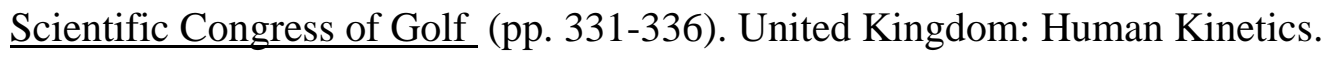


Lampsa, M. (1975). Maximal distance of the golf drive: an optimal control study. Journal of Dynamic Systems, Measurement, and Control Transactions. ASME, 362-367.

Mather, J. S. B., Jowett, S. (1998). The effect of centrifugal stiffening on the bending stiffness of a golf shaft. In M. R. Farrally, \& A. J. Cochran (Ed.), Science and Golf III: Proceedings of the World Scientific Congress of Golf (pp. 515-521). United Kingdom: Human Kinetics.

Mather, J. S. B., Smith, M. J., Jowett, S., Gibson, K. A. H., \& Moynihan, D. (2000). Application of a photogrammetric technique to golf club evaluation. $\underline{\text { Sports }}$ Engineering, 3, 37-47.

Mather, J. S. B., \& Cooper, M. A. J. (1994). The attitude of the shaft during the swing of golfers of different ability. In M. R. Farrally, \& A. J. Cochran (Ed.), Science and Golf II: Proceedings of the World Scientific Congress of Golf (pp. 271-277). United Kingdom: E \& FN Spon.

Miao, T., Watari, M., Kawaguchi, M., \& Ikeda, M. (1998). A study of clubhead speed as a function of grip speed for a variety of shaft flexibility. In M. R. Farrally, \& A. J. Cochran (Ed.), Science and Golf III: Proceedings of the World Scientific Congress of Golf (pp. 554-561). United Kingdom: Human Kinetics. 
Miura, K. (2001). Parametric acceleration - the effect of inward pull of the golf club at impact stage. Sports Engineering, 4, 75-86.

Michalewicz, Z. (1996). Genetic algorithms + data structures = evolution programs. New York: Springer.

Milne, R. D., \& Davis, J. P. (1992). The role of the shaft in the golf swing. Journal of Biomechanics, 25, 975-983.

Mitiguy, P. C., \& Kane, T. R. (1996). Motion variables leading to efficient equations of motion. The International Journal of Robotics Research, 15, 522-532.

Neal, R. J., \& Wilson, B. D. (1985). 3D kinematics and kinetics of the golf swing. International Journal of Sports Biomechanics, 1, 221-232.

Okubo, N. \& Simada, M. (1990). Application of CAE (computer aided engineering) to golf club dynamics. In A. J. Cochran (Ed.), Science and Golf: Proceedings of the First World Scientific Congress of Golf (pp. 270-273). United Kingdom: E \& FN Spon.

Pelz, D. (1998). Dave Pelz short game bible. USA: Human Kinetics. 
Press, W., Teukolsky, S., Vetterling, W. \& Flannery, B. (1992). Numerical recipes FORTRAN: the art of scientific computing, $2^{\text {nd }}$ Ed. New York: Cambridge University Press.

Sprigings, E. J. (1986). Simulation of the force enhancement phenomenon in muscle. Computers in Biology and Medicine, 16, 423-430.

Sprigings, E. J., \& Neal, R. (2000). An insight into the importance of wrist torque in driving the golfball: a simulation study. Journal of Applied Biomechanics, 16, 356-366.

United States Golf Association. (2004). Rules of golf. Canada: Royal Canadian Golf Association.

Van Gheluwe, B., Deporte, E., \& Ballegeer, K. (1990). The influence of the use of graphite shafts on golf performance and swing kinematics. In A. J. Cochran (Ed.), Science and Golf: Proceedings of the First World Scientific Congress of Golf (pp. 258263). United Kingdom: E \& FN Spon.

Vaughan, C. L. (1981). A three-dimensional analysis of the forces and torques applied by a golfer during the downswing. In A. Morecki, K. Fidelus, K. Kedzior, \& A. Wit (Ed.), International Series of Biomechanics VII-B (pp. 325-331). Poland: University Park Press. 
Williams, D. (1967). The dynamics of the golf swing. Quarterly Journal of Mechanics and Applied Mathematics, 20, 247-264.

Wilkie, D. R. (1949). The relation between force and velocity in human muscle. Journal of Physiology, 110, 249-280.

Williams, K. R., \& Sih, B. L. (2002). Changes in golf clubface orientation following impact with the ball. Sports Engineering, 5, 65-80.

Winfield, D. C., \& Tan, T. E. (1994). Optimization of clubhead loft and swing elevation angles for maximum distance of a golf drive. Computers and Structures, 53, $19-25$.

Yamaguchi, G. T. (2001). Dynamic modeling of musculoskeletal motion. A vectorized approach for biomechanical analysis in three dimensions. USA: Kluwer Academic Publishers.

Yeadon, M. R. (1990). The simulation of aerial movement: II. A mathematical inertia model of the human body. Journal of Biomechanics, 23, 67-74.

Zatsiorsky, V. M. (2002). Kinetics of human motion. Ontario: Human Kinetics. 


\section{APPENDIX A}

\section{Consent Form}

Ethical Approval 


\section{UNIVERSITY OF SASKATCHEWAN BEHAVIOURAL RESEARCH ETHICS BOARD}

http://www.usask.ca/research/ethics.shtml

NAME: Eric Sprigings

BSC\#: 02- 690

DATE: November 5, 2002

The University Advisory Committee on Ethics in Behavioural Science Research has reviewed the Application for Ethics Approval for your study "Golf Shaft Behaviour During the Downswing" (02-690).

1. Your PROGRAM OF RESEARCH has been APPROVED subject to the following minor modifications:

- Please explain how you intend to recruit the participants? If through an organization, then a cover letter is needed.

2. Please send one copy of your revisions to the Office of Research Services for our records. Please highlight or underline any changes made when resubmitting.

3. The term of this approval is for 5 years.

4. This letter serves as your certificate of approval, effective as of the time that you have

completed the requested modifications. If you require a letter of unconditional approval, please so indicate on your reply, and one will be issued to you.

5. Any significant changes to your proposed study should be reported to the Chair for Committee consideration in advance of its implementation.

6. This approval is valid for five years on the condition that a status report form is submitted annually to the Chair of the Committee. This certificate will automatically be invalidated if a status report form is not received within one month of the anniversary date. Please refer to the website for further instructions:

http://www.usask.ca/research/ethics.shtml

I wish you a successful and informative study.

Dr. John Rigby for

Dr. Valerie Thompson, Chair

Behavioural Research Ethics Board

$\mathrm{VT} / \mathrm{ck}$ 


\section{CONSENT FORM}

You are invited to participate in a study entitled Golf Club Shaft Behaviour During the Downswing. Please read this form carefully, and feel free to ask any questions.

Researchers: Dr. Eric Sprigings (Ph.D.) (966-6481) and Sasho Mackenzie (Ph.D. Candidate) (966-2328), College of Kinesiology, University of Saskatchewan.

Purpose and Procedure: The purpose of this study is to determine how the shaft of a golf club bends during the downswing in golf. Prior to data collection, participants will undergo a warm-up consisting of sub-maximal golf swings and golf specific stretches. Participants will perform their normal golf swing, four times, with each of three types of driver. A high-speed video camera will capture the motion of the club during the downswing, so as to determine the behaviour of the shaft. The total time required by a participant will be approximately 30 minutes.

Potential Risks and Benefits: There are no potential risks associated with the study. Participation may help develop new strategies for golf club design, but this is not guaranteed.

Storage of Data: Dr. Sprigings will store all data collected during this study in a locked office at the University of Saskatchewan for a minimum of five years.

Confidentiality: Attempts will be made to present the findings of this study in a journal article or conference presentation. Individual participant identity will not be revealed.

Right to Withdraw: You may withdraw from the study for any reason, at any time, without penalty of any sort. If you withdraw from the study at any time, any data that you have contributed will be destroyed.

Questions: If you have any questions concerning the study, please feel free to ask at any point; you are also free to contact the researchers at the numbers provided above if you have questions at a later time. This study has been approved on ethical grounds by the University of Saskatchewan Behavioural Sciences Research Ethics Board on May 7, 2003. Any questions regarding your rights as a participant may be addressed to that committee through the Office of Research Services (966-2084).

Consent to Participate: I have read and understood the description provided above; I have been provided with an opportunity to ask questions and my questions have been answered satisfactorily. I consent to participate in the study described above, understanding that I may withdraw this consent at any time. A copy of this consent form has been given to me for my records.

(Signature of Participant)

(Date)

(Signature of Researcher)

(Date) 


\section{APPENDIX B}

Inertial Properties of Simulated Club Segments 
The following calculations detail the principal moments of inertia for a standard driver designed in 2001 if it was divided up into 12 segments. The first 11 segments were each $10 \mathrm{~cm}$ in length and were modelled as circular cylindrical shells. The $12^{\text {th }}$ segment was the clubhead which was modelled as a semi-ellipsoid. These 12 segments were then combined in groups of three. This resulted in four club segments. The principal moments of inertia, masses, centers of mass, and lengths of these 4 segments were determined using Autolev ${ }^{\circledR}$.

\section{Segment 1}

Segment 1 represents the first $10 \mathrm{~cm}$ of the grip end of the club in addition to the hand of the golfer. Based on regression equations from Zatsiorsky (2002), a hand mass of $0.477 \mathrm{~kg}$ was added to the measured mass of the first club segment of $0.03465 \mathrm{~kg}$. This gave the following parameters for the first segment.

$$
\begin{aligned}
& \mathrm{m}_{1}=0.51165 \mathrm{~kg} \\
& \mathrm{~L}_{1}=0.1 \mathrm{~m} \\
& \mathrm{r}_{1}=0.035 \mathrm{~m} \text { (radius of cylinder) } \\
& \mathrm{I} \_\mathrm{x}=\mathrm{I} \_\mathrm{y}=\left(\mathrm{m}_{1} / 2\right)\left(\mathrm{r}_{1}^{2}\right)+\left(\mathrm{m}_{1} / 12\right)\left(\mathrm{L}_{1}^{2}\right)=0.0007398 \mathrm{~kg}^{*} \mathrm{~m}^{2} \\
& \mathrm{I} \_\mathrm{z}=\mathrm{m}_{1} \mathrm{r}_{1}^{2}=0.0006268 \mathrm{~kg}^{*} \mathrm{~m}^{2}
\end{aligned}
$$

The principal moments of inertia for segments 2 to 11 were all calculated in an identical manner. The masses and radii of each segment decreased in magnitude as the segments became further from the grip end. 


\section{Segment 2}

$$
\begin{aligned}
& \mathrm{m}_{2}=0.015 \mathrm{~kg} \\
& \mathrm{~L}_{2}=0.1 \mathrm{~m} \\
& \mathrm{r}_{2}=0.01 \mathrm{~m} \quad \text { radius of cylinder } \\
& \mathrm{I} \_\mathrm{x}=\mathrm{I} \_\mathrm{y}=\left(\mathrm{m}_{2} / 2\right)\left(\mathrm{r}_{2}{ }^{2}\right)+\left(\mathrm{m}_{2} / 12\right)\left(\mathrm{L}_{2}{ }^{2}\right)=0.00001325 \mathrm{~kg}^{*} \mathrm{~m}^{2} \\
& \mathrm{I} \_\mathrm{z}=\mathrm{m}_{2} \mathrm{r}_{2}{ }^{2}=0.0000015 \mathrm{~kg}^{*} \mathrm{~m}^{2}
\end{aligned}
$$

\section{Segment 3}

$$
\begin{aligned}
& \mathrm{m}_{3}=0.0072 \mathrm{~kg} \\
& \mathrm{~L}_{3}=0.1 \mathrm{~m} \\
& \mathrm{r}_{3}=0.0075 \mathrm{~m} \text { radius of cylinder } \\
& \mathrm{I} \_\mathrm{x}=\mathrm{I} \_\mathrm{y}=\left(\mathrm{m}_{3} / 2\right)\left(\mathrm{r}_{3}{ }^{2}\right)+\left(\mathrm{m}_{3} / 12\right)\left(\mathrm{L}_{3}{ }^{2}\right)=0.0000062 \mathrm{~kg}^{*} \mathrm{~m}^{2} \\
& \mathrm{I} \_\mathrm{z}=\mathrm{m}_{3} \mathrm{r}_{3}{ }^{2}=0.0000004 \mathrm{~kg}^{*} \mathrm{~m}^{2}
\end{aligned}
$$

\section{Segment 4}

$$
\begin{aligned}
& \mathrm{m}_{4}=0.0071 \mathrm{~kg} \\
& \mathrm{~L}_{4}=0.1 \mathrm{~m} \\
& \mathrm{r}_{4}=0.007 \mathrm{~m} \text { radius of cylinder } \\
& \mathrm{I} \_\mathrm{x}=\mathrm{I} \_\mathrm{y}=\left(\mathrm{m}_{4} / 2\right)\left(\mathrm{r}_{4}^{2}\right)+\left(\mathrm{m}_{4} / 12\right)\left(\mathrm{L}_{4}^{2}\right)=0.0000061 \mathrm{~kg}^{*} \mathrm{~m}^{2} \\
& \mathrm{I} \_\mathrm{z}=\mathrm{m}_{4} \mathrm{r}_{4}{ }^{2}=0.00000035 \mathrm{~kg}^{*} \mathrm{~m}^{2}
\end{aligned}
$$

\section{Segment 5}

$$
\mathrm{m}_{5}=0.0070 \mathrm{~kg}
$$


$\mathrm{L}_{5}=0.1 \mathrm{~m}$

$\mathrm{r}_{5}=0.0065 \mathrm{~m}$ radius of cylinder

$\mathrm{I} \_\mathrm{x}=\mathrm{I} \_\mathrm{y}=\left(\mathrm{m}_{5} / 2\right)\left(\mathrm{r}_{5}{ }^{2}\right)+\left(\mathrm{m}_{5} / 12\right)\left(\mathrm{L}_{5}{ }^{2}\right)=0.00000598 \mathrm{~kg}^{*} \mathrm{~m}^{2}$

$\mathrm{I} \_\mathrm{z}=\mathrm{m}_{5} \mathrm{r}_{5}{ }^{2}=0.00000030 \mathrm{~kg}^{*} \mathrm{~m}^{2}$

\section{Segment 6}

$\mathrm{m}_{6}=0.0069 \mathrm{~kg}$

$\mathrm{L}_{6}=0.1 \mathrm{~m}$

$\mathrm{r}_{6}=0.0060 \mathrm{~m}$ radius of cylinder

$\mathrm{I} \_\mathrm{x}=\mathrm{I} \_\mathrm{y}=\left(\mathrm{m}_{6} / 2\right)\left(\mathrm{r}_{6}{ }^{2}\right)+\left(\mathrm{m}_{6} / 12\right)\left(\mathrm{L}_{6}{ }^{2}\right)=0.00000587 \mathrm{~kg} * \mathrm{~m}^{2}$

$\mathrm{I} \_\mathrm{z}=\mathrm{m}_{6} \mathrm{r}_{6}{ }^{2}=0.00000025 \mathrm{~kg} * \mathrm{~m}^{2}$

\section{Segment 7}

$$
\begin{aligned}
& \mathrm{m}_{7}=0.0068 \mathrm{~kg} \\
& \mathrm{~L}_{7}=0.1 \mathrm{~m} \\
& \mathrm{r}_{7}=0.0055 \mathrm{~m} \text { radius of cylinder } \\
& \mathrm{I} \_\mathrm{x}=\mathrm{I} \_\mathrm{y}=\left(\mathrm{m}_{7} / 2\right)\left(\mathrm{r}_{7}^{2}\right)+\left(\mathrm{m}_{7} / 12\right)\left(\mathrm{L}_{7}^{2}\right)=0.00000577 \mathrm{~kg}^{*} \mathrm{~m}^{2} \\
& \mathrm{I} \_\mathrm{z}=\mathrm{m}_{7} \mathrm{r}_{7}^{2}=0.00000021 \mathrm{~kg}^{*} \mathrm{~m}^{2}
\end{aligned}
$$

\section{Segment 8}

$$
\begin{aligned}
& \mathrm{m}_{8}=0.0067 \mathrm{~kg} \\
& \mathrm{~L}_{8}=0.1 \mathrm{~m} \\
& \mathrm{r}_{8}=0.0050 \mathrm{~m} \text { radius of cylinder }
\end{aligned}
$$




$$
\begin{aligned}
& \mathrm{I} \_\mathrm{x}=\mathrm{I} \_\mathrm{y}=\left(\mathrm{m}_{8} / 2\right)\left(\mathrm{r}_{8}{ }^{2}\right)+\left(\mathrm{m}_{8} / 12\right)\left(\mathrm{L}_{8}{ }^{2}\right)=0.00000567 \mathrm{~kg} * \mathrm{~m}^{2} \\
& \mathrm{I} \_\mathrm{z}=\mathrm{m}_{8} \mathrm{r}_{8}{ }^{2}=0.00000017 \mathrm{~kg} * \mathrm{~m}^{2}
\end{aligned}
$$

\section{Segment 9}

$\mathrm{m}_{9}=0.0066 \mathrm{~kg}$

$\mathrm{L}_{9}=0.1 \mathrm{~m}$

$\mathrm{r}_{9}=0.0045 \mathrm{~m}$ radius of cylinder

$\mathrm{I} \_\mathrm{x}=\mathrm{I} \_\mathrm{y}=\left(\mathrm{m}_{9} / 2\right)\left(\mathrm{rg}_{9}{ }^{2}\right)+\left(\mathrm{m}_{9} / 12\right)\left(\mathrm{L}_{9}{ }^{2}\right)=0.00000557 \mathrm{~kg}^{*} \mathrm{~m}^{2}$

$\mathrm{I} \_\mathrm{z}=\mathrm{m}_{9} \mathrm{r}_{9}^{2}=0.00000013 \mathrm{~kg}^{*} \mathrm{~m}^{2}$

\section{Segment 10}

$\mathrm{m}_{10}=0.0065 \mathrm{~kg}$

$\mathrm{L}_{10}=0.1 \mathrm{~m}$

$\mathrm{r}_{10}=0.0040 \mathrm{~m}$ radius of cylinder

$\mathrm{I} \_\mathrm{x}=\mathrm{I} \_\mathrm{y}=\left(\mathrm{m}_{10} / 2\right)\left(\mathrm{r}_{10}{ }^{2}\right)+\left(\mathrm{m}_{10} / 12\right)\left(\mathrm{L}_{10}{ }^{2}\right)=0.00000547 \mathrm{~kg}^{*} \mathrm{~m}^{2}$

$\mathrm{I} \_\mathrm{z}=\mathrm{m}_{10} \mathrm{r}_{10}{ }^{2}=0.00000010 \mathrm{~kg}^{*} \mathrm{~m}^{2}$

\section{Segment 11}

$\mathrm{m}_{11}=0.0064 \mathrm{~kg}$

$\mathrm{L}_{11}=0.1 \mathrm{~m}$

$\mathrm{r}_{11}=0.0035 \mathrm{~m}$ radius of cylinder

$\mathrm{I} \_\mathrm{x}=\mathrm{I} \_\mathrm{y}=\left(\mathrm{m}_{11} / 2\right)\left(\mathrm{r}_{11}{ }^{2}\right)+\left(\mathrm{m}_{11} / 12\right)\left(\mathrm{L}_{11}{ }^{2}\right)=0.00000537 \mathrm{~kg} * \mathrm{~m}^{2}$

$\mathrm{I} \_\mathrm{z}=\mathrm{m}_{11} \mathrm{r}_{11}{ }^{2}=0.00000008 \mathrm{~kg} * \mathrm{~m}^{2}$ 


\section{Segment 12}

Segment 12 represents the clubhead. The following equations are based on modelling the clubhead as a semi-ellipsoid.

$\mathrm{m}_{12}=0.2 \mathrm{~kg}$

$\mathrm{x}=0.11 \mathrm{~m} \quad$ diameter of clubhead along the $\mathrm{x}$-axis

$\mathrm{y}=0.09 \mathrm{~m} \quad$ diameter of clubhead along the $\mathrm{y}$-axis

$\mathrm{z}=0.05 \mathrm{~m} \quad$ diameter of clubhead along the $\mathrm{z}$-axis.

$\mathrm{I} \_\mathrm{x}=\left(\mathrm{m}_{12} / 5\right)\left(\mathrm{z}^{2}+(19 / 64) \mathrm{y}^{2}\right)=0.000196 \mathrm{~kg}^{*} \mathrm{~m}^{2}$

$\mathrm{I} \_\mathrm{y}=\left(\mathrm{m}_{12} / 5\right)\left(\mathrm{z}^{2}+\mathrm{x}^{2}\right)=0.000584 \mathrm{~kg}^{*} \mathrm{~m}^{2}$

$\mathrm{I} \_\mathrm{z}=\left(\mathrm{m}_{12} / 5\right)\left(\mathrm{z}^{2}+(19 / 64) \mathrm{y}^{2}\right)=0.000580 \mathrm{~kg}^{*} \mathrm{~m}^{2}$

The following is the Autolev ${ }^{\odot}$ code that was used to generate the moments of inertia and other parameter values for a 4 segment golf club based on the 12 segment club just defined.

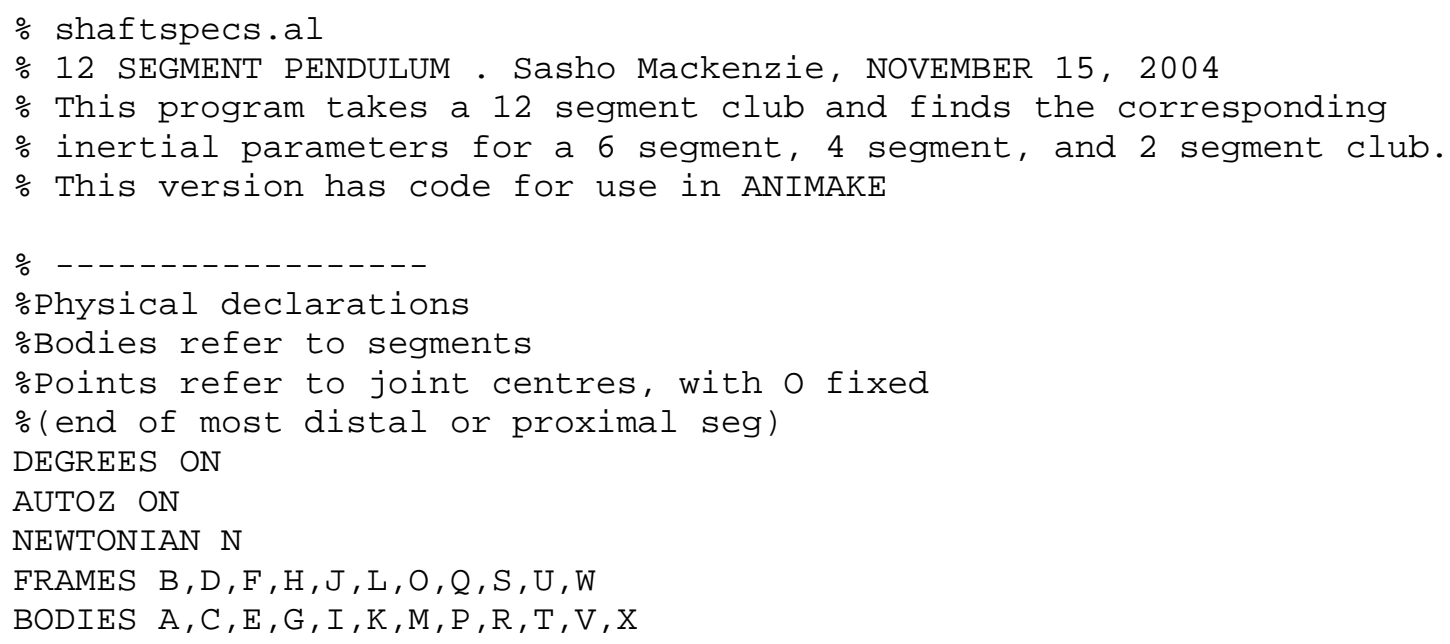




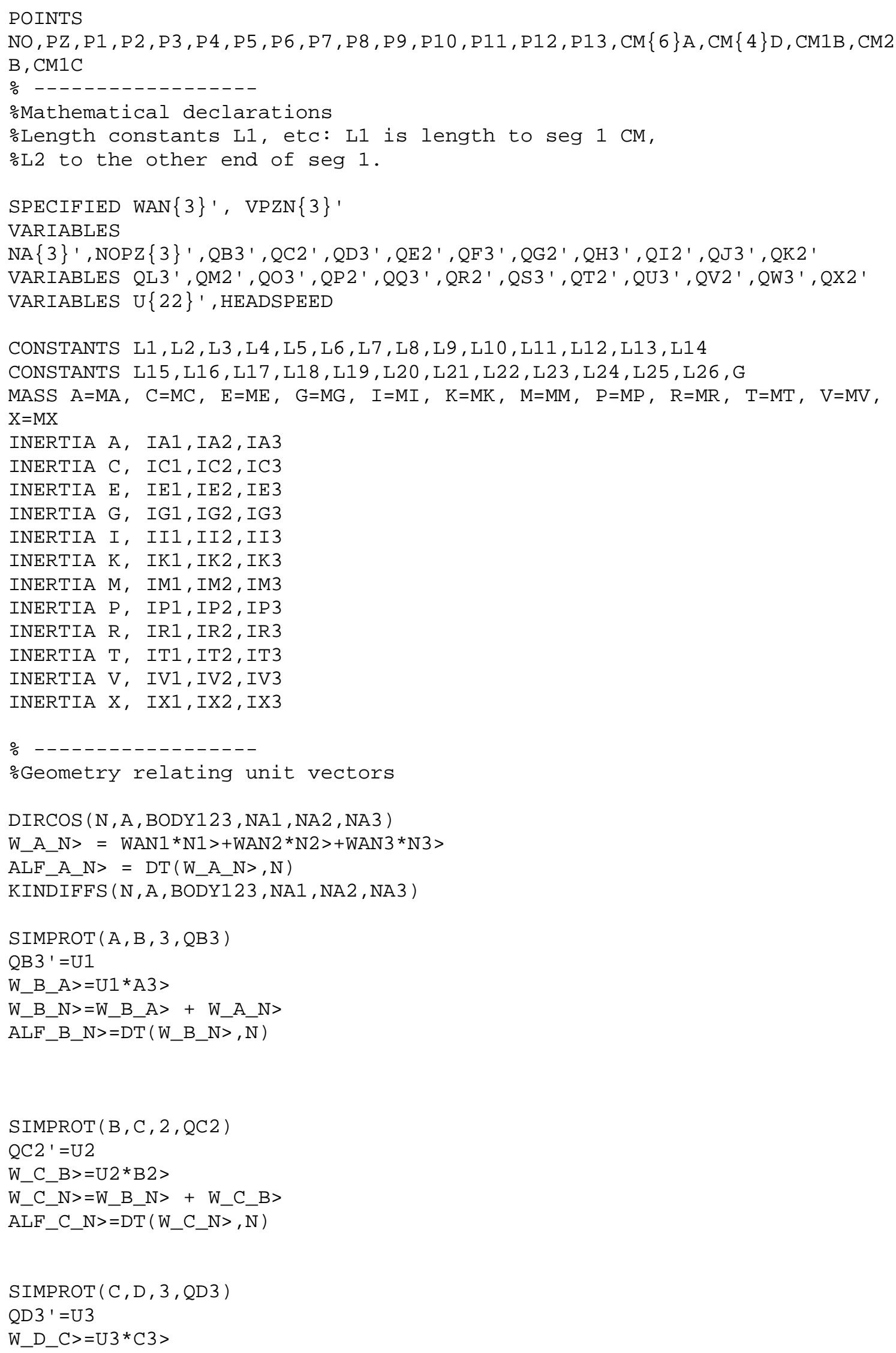




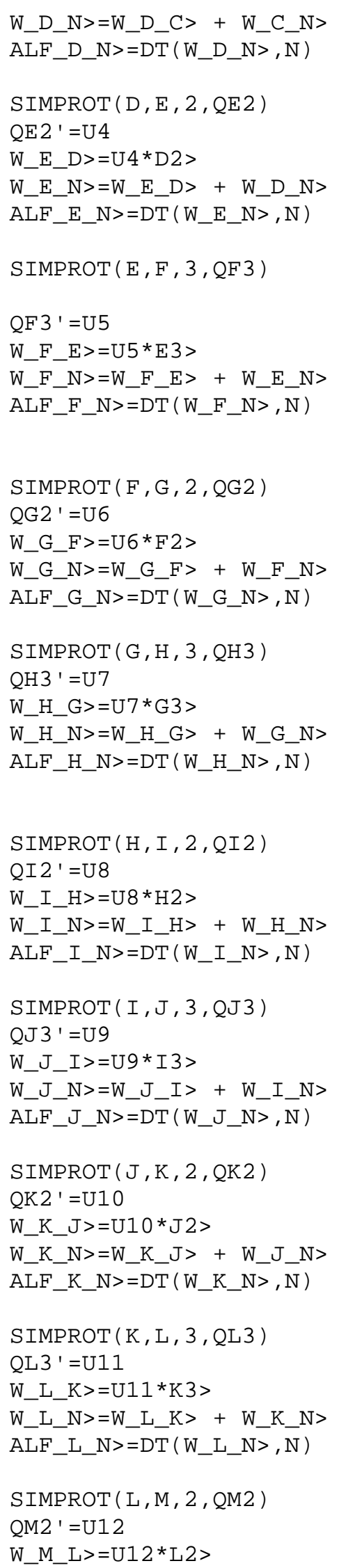




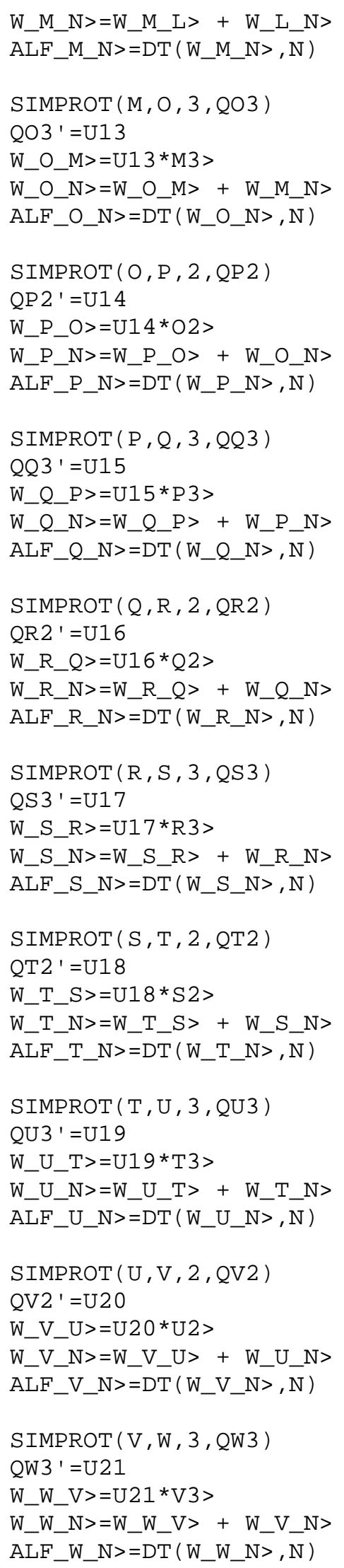




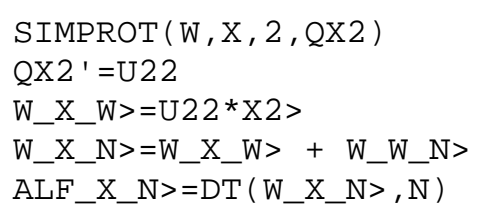

\%use vector addition to define 2 nd seg points relative to origin P_NO_AO >=P_NO_PZ> + P_PZ_AO>

P_NO_P1>=P_NO_AO> + P_AO_P1>

$P \_N O \_C 0>=P \_N 0 \_P 1>+P \_P 1 \_C 0>$

$P \_N O \_P 2>=P \_N O \_P 1>+P \_P 1 \_P 2>$

$P \_N O \_E 0>=P \_N O \_P 2>+P \_P 2 \_E 0>$

P_NO_P3 $>=P \_N O \_P 2>+P \_P 2 \_P 3>$

P_NO_GO $>=$ P_NO_P3>+P_P3_GO> 


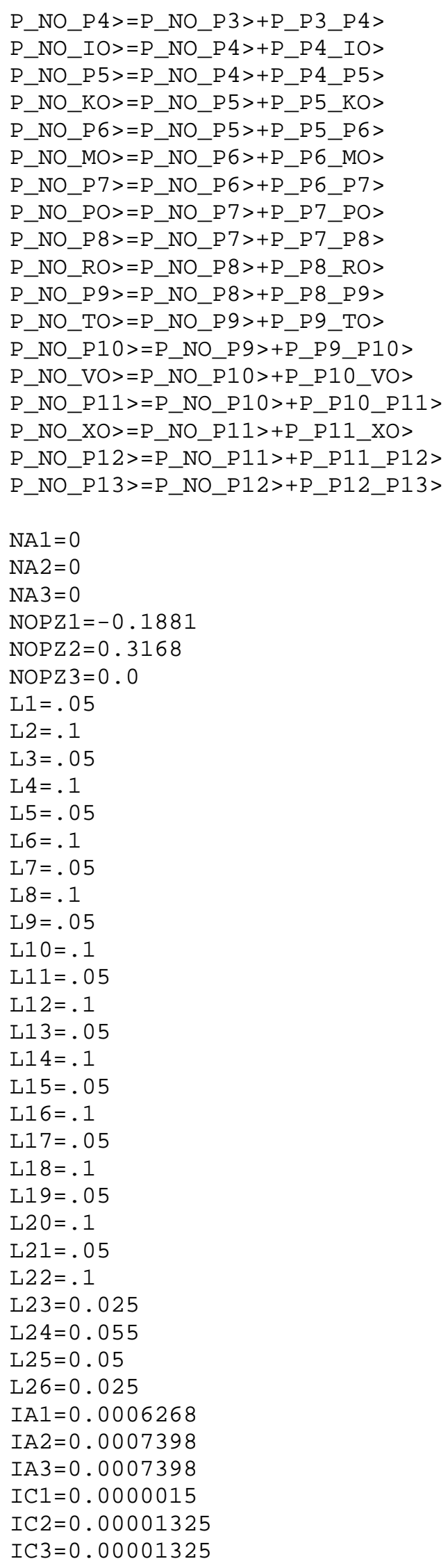




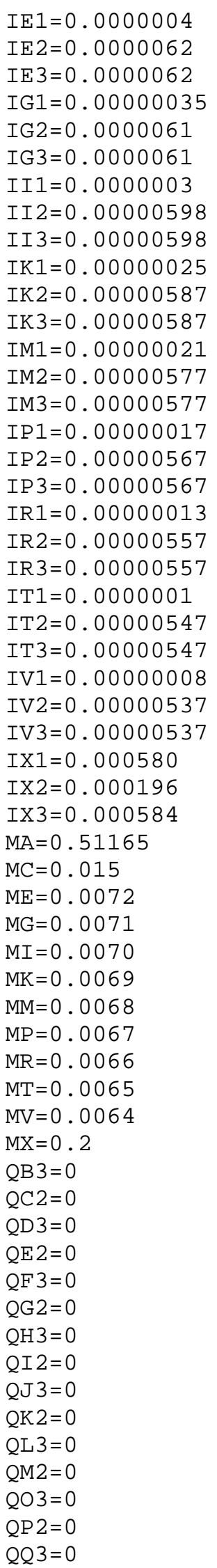




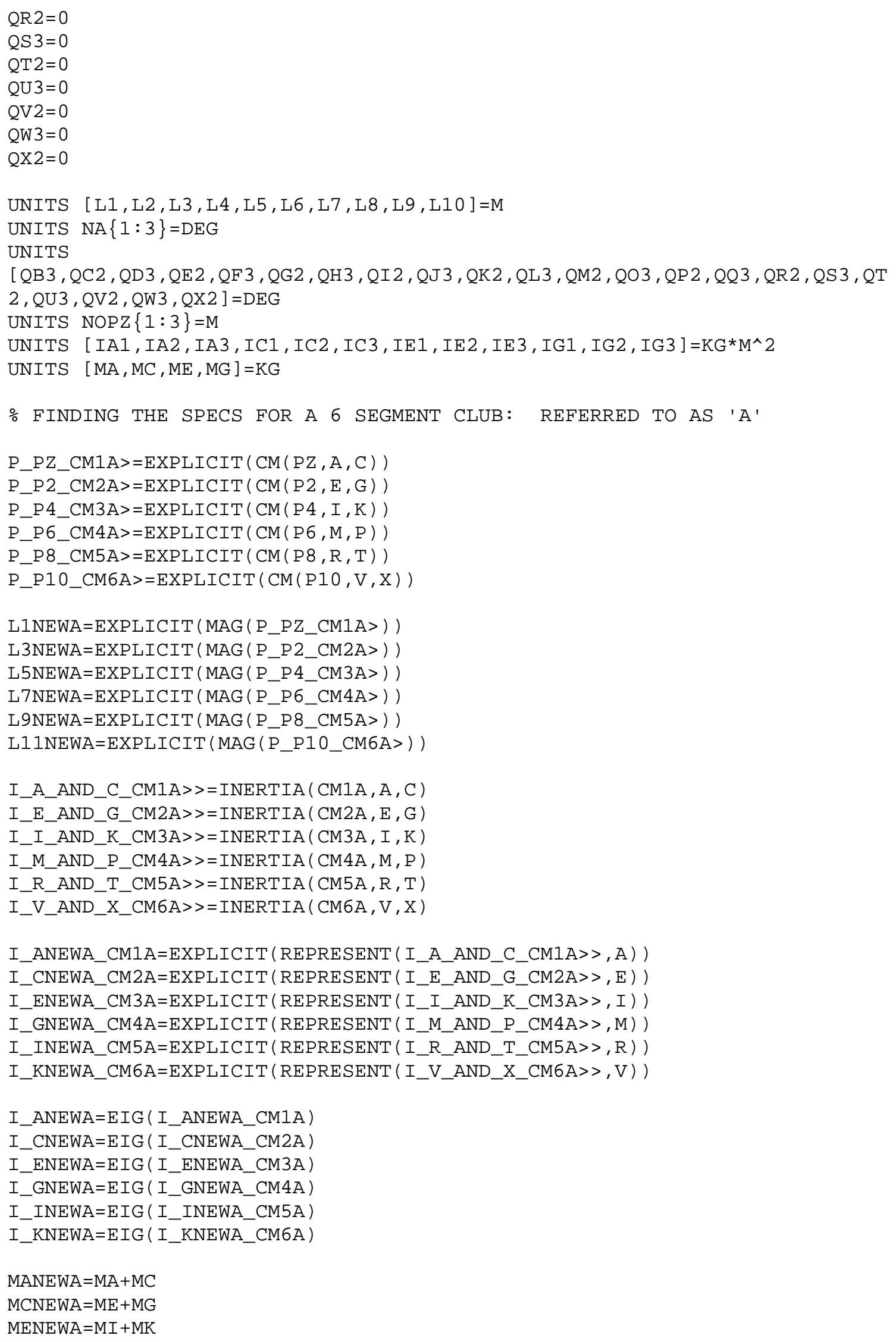




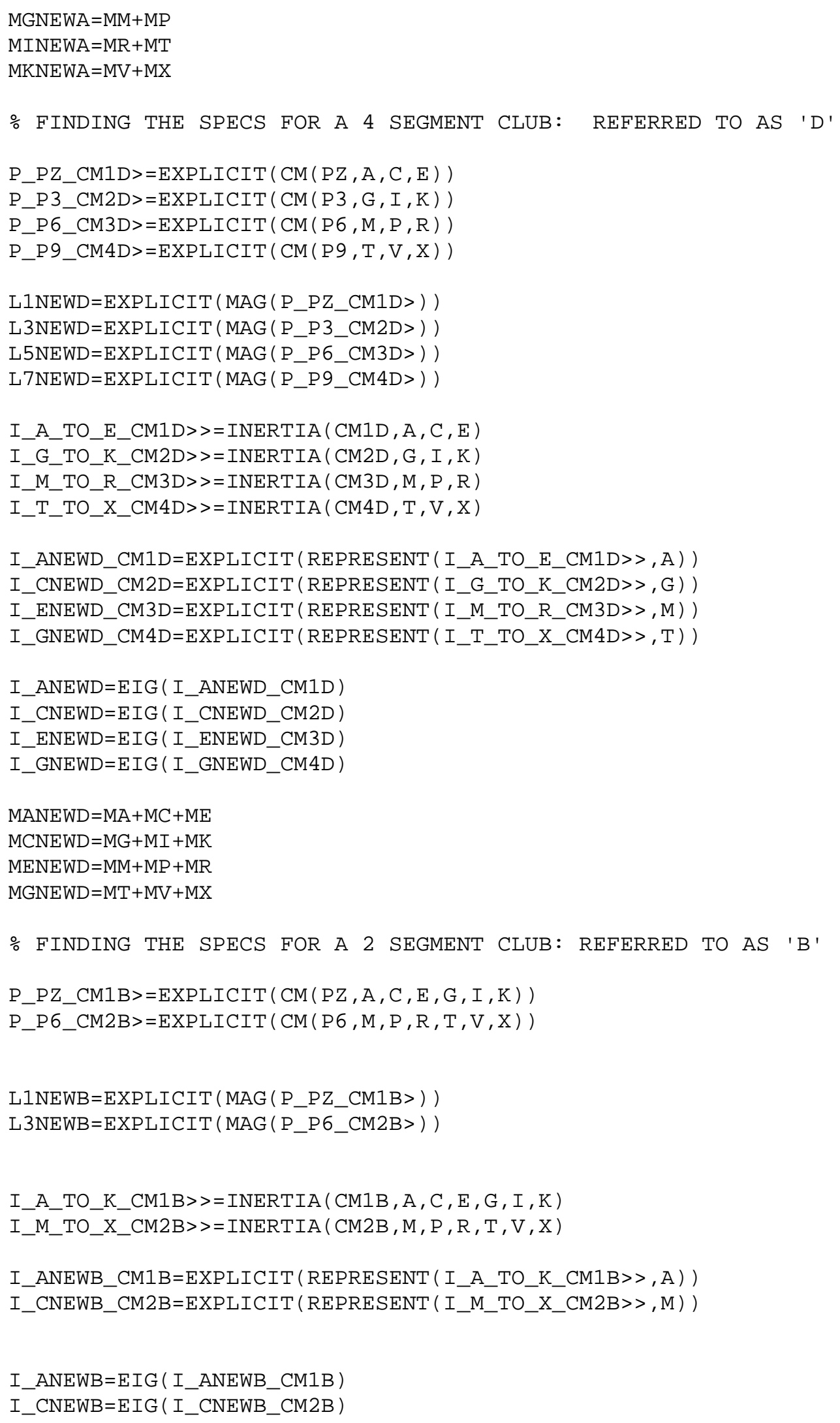


$M A N E W B=M A+M C+M E+M G+M I+M K$

$M C N E W B=M M+M P+M R+M T+M V+M X$

\% FINDING THE SPECS FOR A 1 SEgMENT CLUB: REFERRED TO AS 'C'

\%P_PZ_CM1C >=EXPLICIT (CM(PZ, A, C, E, G, I, K, M, P, R, T, V , X ) )

\%L1NEWC $=$ EXPLICIT $\left(\right.$ MAG $\left.\left(P \_P Z \_C M 1 C>\right)\right)$

\%I_A_TO_X_CM1C >>=INERTIA(CM1C,A, C, E, G , I , K , M , P, R, T, V , X)

\%I_ANEWC_CM1C=EXPLICIT (REPRESENT (I_A_TO_X_CM1C >>,A ) )

\%I_ANEWC=EIG ( I_ANEWC_CM1C $)$

\%MANEWC $=M A+M C+M E+M G+M I+M K+M M+M P+M R+M T+M V+M X$

SAVE SHAFTSPECS.ALL 
APPENDIX C

Autolev ${ }^{\circledR}$ Code: 3DGOLFG.AL 
$\%$ FILE NAME:

$\%$ CREATED BY:

$\%$ LAST MODIFIED:

$\%$

$\%$ This program is used to generate the 3D dynamical equations

$\%$ for a 6 segment golf-club model. The golfer consists of a torso

$\%$ and arm segment, while the club consists of 4 segments.

$\%$ Five other similar programs were created to develope equations

$\%$ with slightly different constraints.

$\%$

\%Physical declarations

\%Bodies refer to segments

\%Points refer to joint centres, with O fixed

DEGREES ON

AUTOZ ON

NEWTONIAN N

BODIES A,B,C,D,E,F

FRAMES TILT,INTAB,INTCD,INTDE,INTEF,HEAD,FACE,GLOBAL

POINTS O,P1,P2,P2B,P3,P4,P5,P6,P7,ALLCM

BODIES BALL

$\%$

\%Mathematical declarations

CONSTANTS L1,L2,L3,L4,L5,L6,L7,L8,L9,L10,L11,L12,L13,L14,L15,L16

CONSTANTS TNA_ON,TNA_GO,TAB1_ON,TAB3_ON,TAB3_GO,TBC3_ON

CONSTANTS TBC3_GO,T1_STARTA, T2_STARTA,T3_STARTA,T4_STARTA CONSTANTS FLAGT1A,FLAGT2A, FLAGT3A,FLAGT4A, TNAM,TAB1DYN, CONSTANTS TAB3DYN,TAB3M,TBC3DYN,TBC3M,STP,TMAX,WTRUNK CONSTANTS TAB1M W1MAX,GG,WFOREARM,W4MAX,T1,T2,T3,T4,SW,G4 CONSTANTS WARM, W2MAX,WCLUB,W3MAX CONSTANTS K1,K2,K3,D,G

SPECIFIED VBALLN $\{3\}$ ',WBALLN3'

VARIABLES U $\{15\}^{\prime}$

VARIABLES QA3', QTILT1', QB1', QB3', QC $\{3\}$ ', QD2', QD3'

VARIABLES QHEAD3,QFACE1,QGLOBAL1, QE2', QE3', QF2'

VARIABLES QF3',NOBALL $\{3\}$ ',QBALL3',QJRF $\{3\}$ ',JRFC1,JRFC2,JRFC3

VARIABLES TNA, TAB1, TAB3, TBC $\{3\}$, HEADSPEED, POP7X, BEND2,BEND3

VARIABLES ELEV,DEV,DROOP,LEAD,TH

VARIABLES TCD3=-K1*QD3-(U5*D), TCD2=-K1*QD2-(U6*D)

VARIABLES TDE3 $=-\mathrm{K} 2 * \mathrm{QE} 3-(\mathrm{U} 7 * \mathrm{D}), \mathrm{TDE} 2=-\mathrm{K} 2 * \mathrm{QE} 2-(\mathrm{U} 8 * \mathrm{D})$

VARIABLES TEF3=-K3*QF3-(U9*D), TEF2=-K3*QF2-(U10*D) 
VARIABLES X1,X2,X3,X4,X5,Y1,Y2,Y3,Y4,Y5,TH1,TH2,TH3,TH4,TORK1 VARIABLES TORK2,TORK3

ZEE_NOT $=[$ TBC1,TBC2,JRFC1,JRFC2,JRFC3 $]$

MASS A=MA, B=MB, C=MC, D=MD, E=ME, F=MF, BALL=MBALL

INERTIA A, IA1,IA2,IA3

INERTIA B, IB1,IB2,IB3

INERTIA C, IC1,IC2,IC3

INERTIA D, ID1,ID2,ID3

INERTIA E, IE1,IE2,IE3

INERTIA F, IF1,IF2,IF3

INERTIA BALL,IBALL3,IBALL3,IBALL3

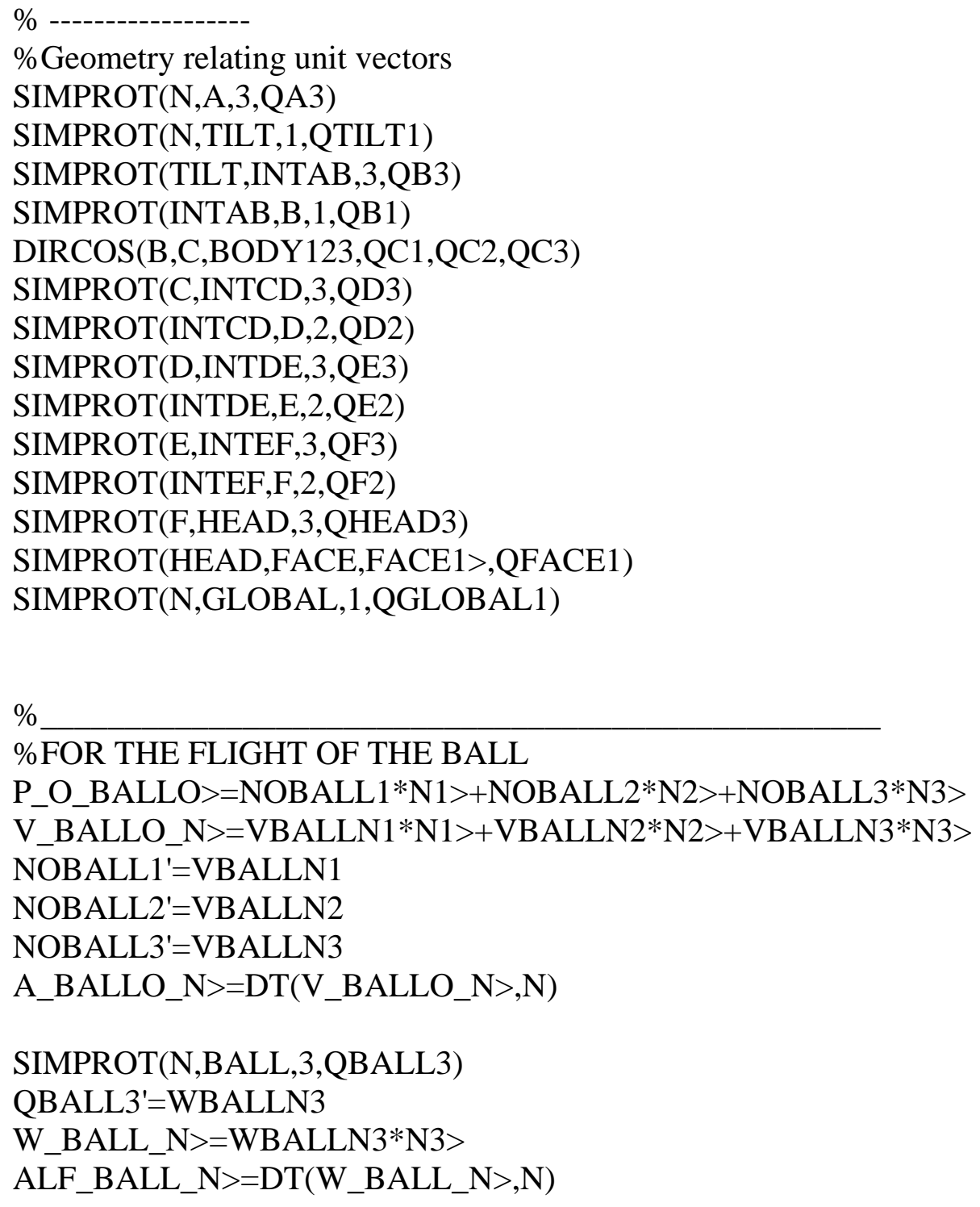


$\%$

\%Position Vectors

\%use lengths and Local Co-ord Sys (LCS) to define

P_O_AO $>=$ L $1 * A 1>$

P_O_P1 $>=$ L2*A1>

P_P1_BO $>=$ L3*B1>

P_P1_P2 $>=L 4 * B 1>$

P_P2_P2B $>=$ QJRF1*N1 $>+$ QJRF2*N2 $>+$ QJRF3*N3>

P_P2B_CO $>=$ L $5 *$ C1>

P_P2B_P3 $>=$ L6 $*$ C1 $>$

P_P3_DO $>=$ L $7 *$ D1 $>$

P_P3_P4 $>=$ L8*D1>

P_P4_EO $>=$ L9 $*$ E1 $>$

P_P4_P5 $>=$ L10*E1>

P_P5_FO $>=$ L11*F1 $>-L 13 * F 2>-L 14 * F 3>$

P_P5_P6 $>=\mathrm{L} 12 * \mathrm{~F} 1>$

P_P6_P7>=-L15*F2>-L16*F3>

P_P7_HEADO $>=0>$

P_HEADO_FACEO $>=0>$

P_O_GLOBALO $>=0>$

P_O_TILTO $>=0>$

P_P1_INTABO $>=0>$

P_P3_INTCDO $>=0>$

P_P4_INTDEO $>=0>$

P_P5_INTEFO $>=0>$

\%use vector addition to define 2nd seg points relative to origin

P_O_BO $>=$ P_O_P1 $>+P$ P1_BO $>$

P_O_P2 $>=P$ OO_P1 $>+P$ P 1 _P2 $>$

P_O_P2B $>=$ P_O_P2 $>+$ P_P2_P2B $>$

P_O_CO $>=$ P_O_P2B $>+P$ P2B_CO $>$

P_O_P3 $>=$ P_O_P2B $>+$ P_P2B_P3 $>$

P_O_DO $>=$ P_O_P3 $>+P$ P3_DO $>$

P_O_P4 $>=$ P_O_P3 $>+P$ P3_P4 $>$

P_O_EO $>=$ P_O_P4 $>+P$ P4_EO $>$

P_O_P5 $>=$ P_O_P4 $>+P$ P4_P5 $>$

P_O_FO $>=$ P_O_P5 $>+P$ P5_FO $>$

P_O_P6 $>=$ P_O_P5 $>+P$ P5_P6 $>$

P_O_P7 $>=$ P_O_P6 $>+P \_P 6 \_P 7>$ 
POP7Y=DOT(P_O_P7>,GLOBAL3>)

$\%$

\% Determing the coordinates of the shaft in the plane of the swing

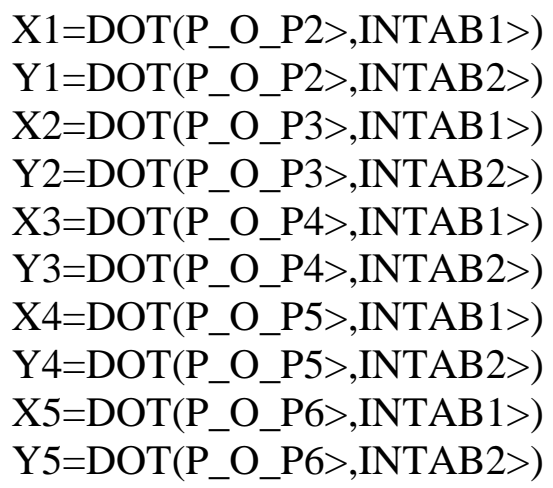

\% Determining the shaft bending angle in the plane of the swing

TH1 $=$ ATAN $($ ABS $(X 1-X 2) / A B S(Y 1-Y 2))$

$\mathrm{TH} 2=\operatorname{ATAN}(\mathrm{ABS}(\mathrm{X} 2-\mathrm{X} 3) / \mathrm{ABS}(\mathrm{Y} 2-\mathrm{Y} 3))$

TH3 $=$ ATAN $($ ABS $(X 3-X 4) / A B S(Y 3-Y 4))$

TH4= ATAN( ABS(X4-X5) / ABS(Y4-Y5) )

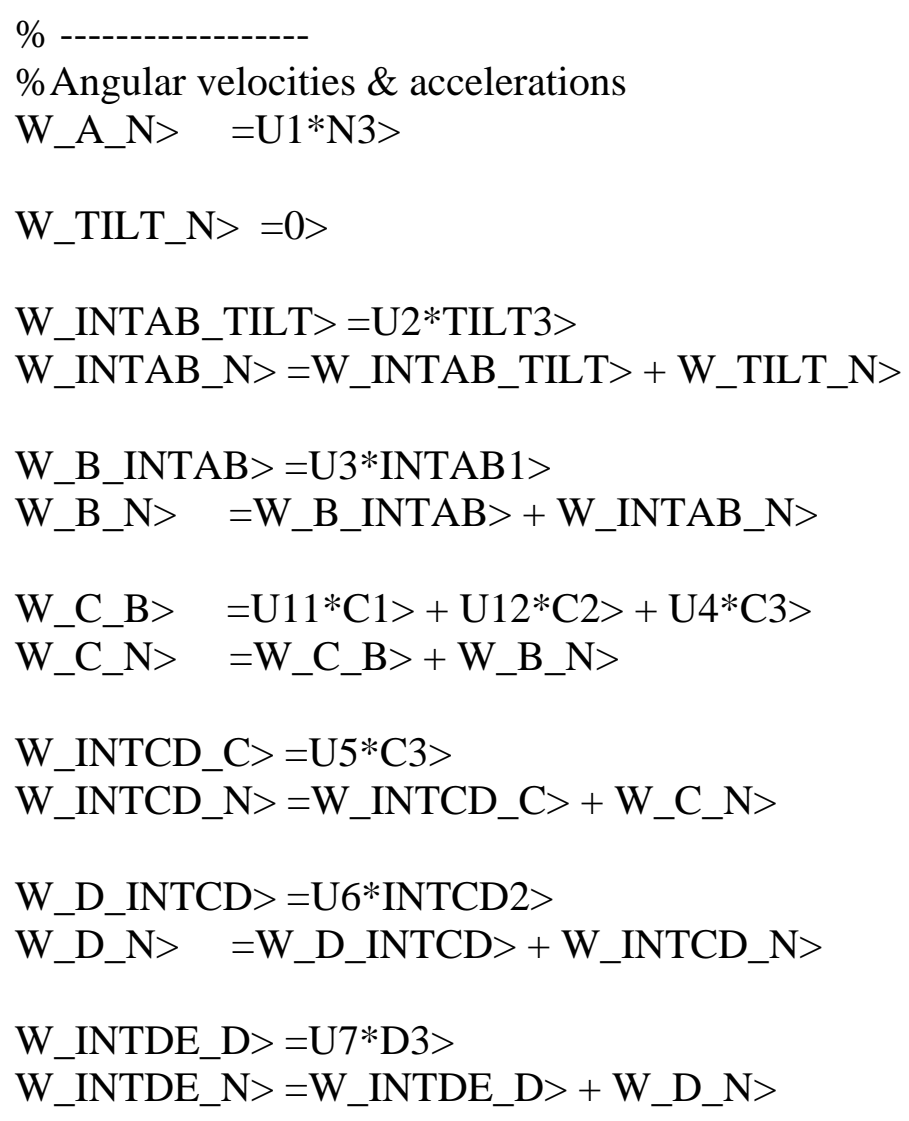

\%Angular velocities $\&$ accelerations

W_A_N $>\quad=\mathrm{U} 1 * \mathrm{~N} 3>$

W_TILT_N $>=0>$

W_INTAB_TILT $>=U 2 * T I L T 3>$

W_INTAB_N $>=$ W_INTAB_TILT $>+$ W_TILT_N $>$

W_B_INTAB $>=$ U3 $*$ INTAB1 $>$

W_B_N $>\quad$ W_B_INTAB $>+$ W_INTAB_N $>$

W_C_B $>\quad=\mathrm{U} 11 * \mathrm{C} 1>+\mathrm{U} 12 * \mathrm{C} 2>+\mathrm{U} 4 * \mathrm{C} 3>$

W_C_N $>$ W_C_B $>+$ W_B_N $>$

W_INTCD_C $>=\mathrm{U} 5 * \mathrm{C} 3>$

W_INTCD_N $>=$ W_INTCD_C $>+$ W_C_N $>$

W_D_INTCD $>=$ U6 $*$ INTCD2 $>$

W_D_N $>$ W_D_INTCD $>+$ W_INTCD_N $>$

W_INTDE_D $>=\mathrm{U} 7 * \mathrm{D} 3>$

W_INTDE_N $>=$ W_INTDE_D $>+$ W_D_N $>$ 


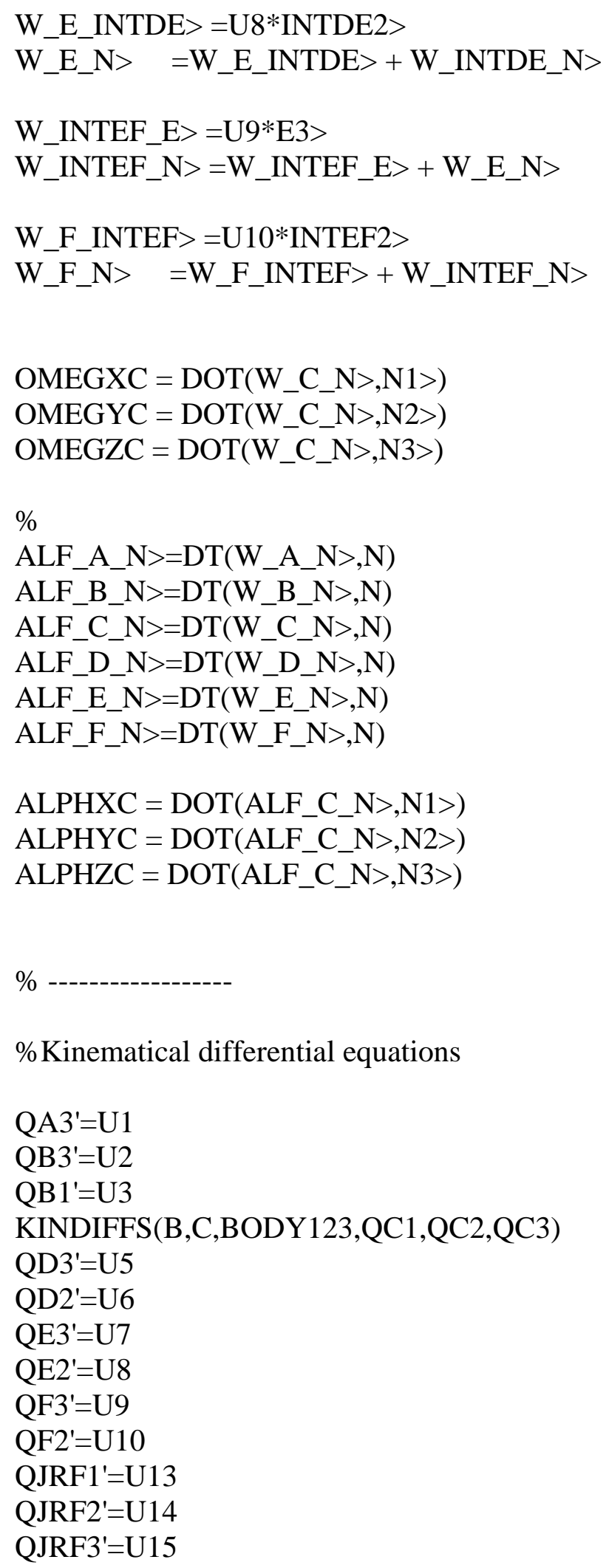




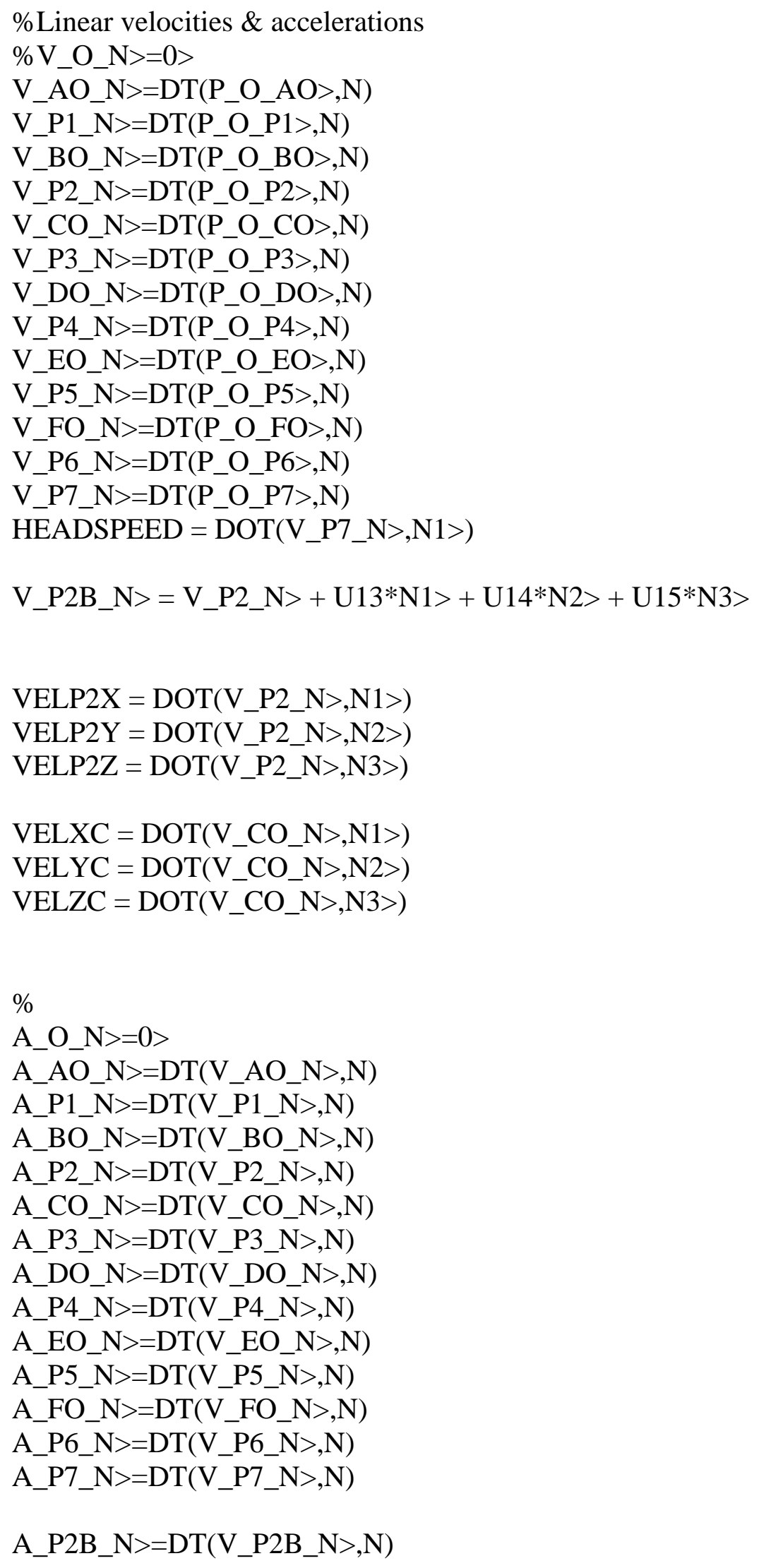




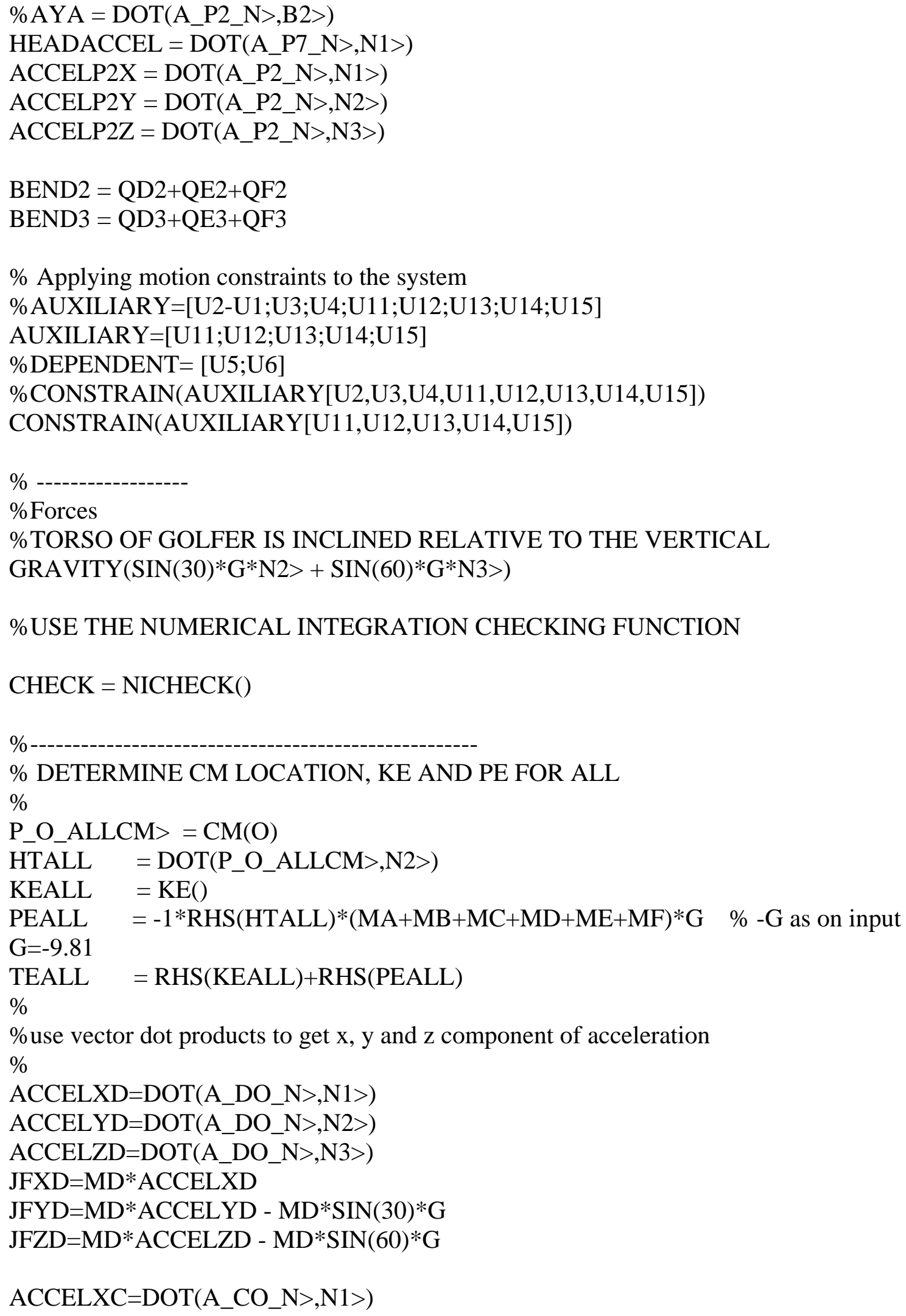




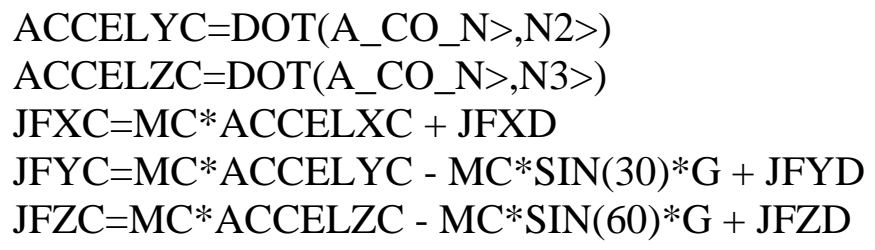

\% Calculating the displacement of the clubhead relative to the grip end

DROOP = DOT $\left(\left(\mathrm{P} \_O \_P 2>-P \_O \_P 6>\right), C 2>\right)$

LEAD = DOT $\left(\left(\mathrm{P} \_O \_P 2>-P \_O \_P 6>\right), C 3>\right)$

\% Calculating the torques AND forces acting on the gripend of the club relative to $\mathrm{N}>$ TORK1 $=$ DOT $(\mathrm{TBC} 1 * \mathrm{C} 1>+\mathrm{TBC} 2 * \mathrm{C} 2>+\mathrm{TBC} 3 * \mathrm{C} 3>$,N1 $>$ )

TORK2 $=$ DOT $(\mathrm{TBC} 1 * \mathrm{C} 1>+\mathrm{TBC} 2 * \mathrm{C} 2>+\mathrm{TBC} 3 * \mathrm{C} 3>$,N2>)

TORK3 $=$ DOT $(\mathrm{TBC} 1 * \mathrm{C} 1>+\mathrm{TBC} 2 * \mathrm{C} 2>+\mathrm{TBC} 3 * \mathrm{C} 3>$,N3 $>)$

FORC1 $1=$ DOT $(J R F C 1 *$ C1 $>+$ JRFC $2 *$ C2 $>+$ JRFC3 $*$ C3 $>, N 1>)$

FORC2 $=$ DOT $(\mathrm{JRFC} 1 * \mathrm{C} 1>+\mathrm{JRFC} 2 * \mathrm{C} 2>+\mathrm{JRFC} 3 * \mathrm{C} 3>$,N2 $>)$

FORC3 $=$ DOT $\left(\right.$ JRFC1 ${ }^{*} \mathrm{C} 1>+$ JRFC $2 * \mathrm{C} 2>+$ JRFC3 $*$ C3 $>$,N3 $>$ )

$\%$

\%Inputs

INPUT TINITIAL $=0.0$, TFINAL $=0.4$, INTEGSTP $=0.001$, PRINTINT $=1$

INPUT ABSERR=1.0E-05, RELERR=1.0E-04

INPUT TNA $=0.0, \mathrm{~K} 1=350, \mathrm{~K} 2=325, \mathrm{~K} 3=300, \mathrm{TAB} 3=0.0, \mathrm{TAB} 1=0.0, \mathrm{TBC} 3=0.0$ INPUT [QB1,QD2,QD3,QE2,QE3,QF2,QF3] =0.0, QA3=270, QTILT1=15, QB3=110, QC3=-110,QHEAD3=-45,QFACE1=10,QGLOBAL1=60

$\%$ Inertial paramenters are taken from Zatsiorsky (2002), Kinetics of Human Motion p.591

$\%$ Values are based on an $80 \mathrm{~kg}$ man with segment lengths the same as mine. See Bodyspecs.al and shaftspecs.al

INPUT L1 $=0.0, \mathrm{~L} 2=0.2$, L3=0.2613, L4 $=0.6$

INPUT L5 $=.05550716, \mathrm{~L} 7=.1490476, \mathrm{~L} 9=.149005, \mathrm{~L} 11=.2174025$

INPUT L12=.225,L13=.05166745,L14=.04697041,L15=.055,L16=.05,[L6,L8,L10]=0.3

INPUT IA $1=0.17256$,IA2 $=0.07052$, IA $3=0.36552$

INPUT IB1 $=0.005806$, IB2 $=0.1076$, IB3 $=0.1096$ 
INPUT IC1 $=0.0006287$, [IC2,IC3] $=0.001181059$

INPUT ID1 $=0.0000009$, [ID2,ID3] $=0.000157931$

INPUT IE1 $=0.00000051$, [IE2,IE3] $=0.0001509901$

INPUT IF1 $=0.0004199793$, IF2 $=0.0006621033, I F 3=0.0008792326$

INPUT MA $=34.613, \mathrm{MB}=3.431$

INPUT MC $=0.53385, \mathrm{MD}=0.021, \mathrm{ME}=0.0201, \mathrm{MF}=0.2129, \mathrm{MBALL}=0.04569$

INPUT G=-9.81

INPUT $\mathrm{D}=10$

INPUT U1 $=-5$

INPUT IBALL3 $=0.0001$

INPUT NOBALL1 $=0.29$, NOBALL2 $=-1.71$, NOBALL3 $=0.203$

INPUT

[TNA_ON,TNA_GO,TAB1_ON,TAB3_ON,TAB3_GO,TBC3_ON,TBC3_GO,T1_ST ARTA] $=0$

INPUT

[T2_STARTA,T3_STARTA,T4_STARTA,FLAGT1A,FLAGT2A,FLAGT3A,FLAGT4 $\mathrm{A}]=0$

INPUT

[TNAM,TAB1DYN,TAB1M,TAB3DYN,TAB3M,TBC3DYN,TBC3M,STP,TMAX,W

TRUNK] $=0$

INPUT [W1MAX,GG,WFOREARM,W4MAX,T1,T2,T3,T4]=0

INPUT [SW,G4,WARM,W2MAX,WCLUB,W3MAX] $=0$

$\%$

\%Outputs

\%each line defines output file FILENAME.1,FILENAME.2,etc.

\%FILENAME.1 is needed for MatLab plotting program SimPlotAL

\%FILENAME.2 is 'standard' AutoLev output file with angular kinematics

OUTPUT T, TNA, TAB1, TAB3, TBC3, TCD2, TCD3, HEADSPEED,

POP7X,POP7Y,CHECK,HEADACCEL

OUTPUT T, QA3, U1, QB3, U2, QB1, U3, QC3, U4, QD3, U5, QD2, U6,

QE3,U7,QE2,U8,QF3,U9,QF2,U10,TH,BEND2, BEND3

OUTPUT T, ELEV, DEV, DROOP, LEAD

OUTPUT T, OMEGXC, OMEGYC, OMEGZC, VELP2X, VELP2Y, VELP2Z

OUTPUT T, ALPHXC, ALPHYC, ALPHZC, ACCELP2X, ACCELP2Y, ACCELP2Z

OUTPUT T,

TBC1,TBC2,TBC3,TORK1,TORK2,TORK3,JRFC1,JRFC2,JRFC3,FORC1,FORC2,F ORC3

OUTPUT

TNA_ON,TNA_GO,TAB1_ON,TAB3_ON,TAB3_GO,TBC3_ON,TBC3_GO,T1_STA RTA

OUTPUT

T2_STARTA,T3_STARTA,T4_STARTA,FLAGT1A,FLAGT2A,FLAGT3A,FLAGT4 A 
OUTPUT

TNAM,TAB1DYN,TAB1M,TAB3DYN,TAB3M,TBC3DYN,TBC3M,STP,TMAX,WT RUNK

OUTPUT W1MAX,GG,WFOREARM,W4MAX,T1,T2,T3,T4

OUTPUT T,SW,G4,WARM,W2MAX,WCLUB,W3MAX

OUTPUT T,X1,X2,X3,X4,X5,Y1,Y2,Y3,Y4,Y5,TH1,TH2,TH3,TH4

$\%$------------------

\%Units

UNITS [L1, L2, L3, L4, L5, L6, L7, L8, L9, L10,L11,L12,L13,L14,L15,L16] = M

UNITS POP1X=M, POP1Y=M, POP2X=M, POP2Y=M, POP3X=M

UNITS

[TNA,TAB1,TAB3,TBC3,TCD2,TCD3,TDE2,TDE3,TEF2,TEF3,TBC1,TBC2,TORK1 ,TORK2,TORK3] = N*M

UNITS

[QA3,QTILT1,QB1,QB3,QC1,QC2,QC3,QD2,QD3,QE2,QE3,QF2,QF3,BEND2,BEN D3,QBALL3] = DEG

UNITS [QHEAD3,QFACE1,QGLOBAL1,ELEV,DEV,TH] = DEG

UNITS [U1, U2, U3, U4, U5, U6, U7, U8, U9, U10] = RAD/S

UNITS [IA1, IA2, IA3, IB1, IB2, IB3, IC1, IC2, IC3, ID1, ID2, ID3] = KG*M^2

UNITS [IE1, IE2, IE3, IF1, IF2, IF3, IBALL3] = KG* $\mathrm{M}^{\wedge} 2$

UNITS [MA, MB, MC, MD, ME, MF, MBALL] = KG

UNITS [NOBALL1,NOBALL2,NOBALL3,QJRF1,QJRF2,QJRF3]=M

UNITS [DROOP,LEAD] $=\mathrm{M}$

UNITS T=S, [G,HEADACCEL $]=\mathrm{M} / \mathrm{S} \wedge 2$

UNITS HEADSPEED $=\mathrm{M} / \mathrm{S}, \mathrm{POP7X}=\mathrm{M}, \mathrm{POP7Y}=\mathrm{M}$

UNITS [JRFC1,JRFC2,JRFC3,FORC1,FORC2,FORC3] $=\mathrm{N}$

$\%$

\%Directions to Autolev

SAVE 3DGOLFG.ALL

CODE DYNAMICS() 3DGOLFG.FOR, SUBS 


\section{APPENDIX D}

Matlab $^{\complement}$ Code: GOLF3D.M 
\% Program Name: Golf3D.m

\% Programmer: $\quad$ Sasho Mackenzie

\% Purpose: $\quad$ Use a genetic algorithm approach to optimize a 3D 6-

$\% \quad$ segment golfer model for maximum clubhead speed.

\% Date Modified: $\quad$ April 11, 2005

popsize $=200 ; \quad \%$ Number of individuals in each generation

maxgens=2000; \% Number of generations that will be evaluated

numnotran $=0 ; \quad \%$ Number of starting individuals that aren't random

$\%$ Non random individuals must be manually included

$\mathrm{m} 1=.00859 ; \quad \%$ Mutation parameter for the 1st third of the generations

$\mathrm{m} 2=.00234 ; \quad \%$ Mutation parameter for the 2nd third of the generations

$\mathrm{m} 3=.00118$; $\quad \%$ Mutation parameter for the 3nd third of the generations

\% Initializing arrays

bigfit $=[$ ]; \% Stores the velocity of best individual of each generation

maxfit $=[$ ]; \% Stores the velocity of the overall best individual

newpop $=[$ ]; \% Stores the mutated individuals that will represent the next generation index $=[$ ];

$\mathrm{c}=[\mathrm{]}$;

$\mathrm{r}=[\mathrm{]}$;

relfit $=[$ ]; \% Stores the relative fitness of each individual

cumfit $=[$ ]; \% Stores the cumulative fitness of eah individual

velocity $=[$ ]; \% Stores the value of the objective function for each individual

vel=[ ];

individual=[ ]; \% Stores the muscle start and go times for each individual

totalfit $=[$ ]; \% Stores the total fitness for all individuals in a specific generation

parents $=[$ ]; \% Stores the individuals that will be mutated to form the next generation newpop $=[$;

tic $\quad \%$ Used to determine the CPU time of the algorithm

for gen=1:maxgens \% Opimization continues for the specified number of generations

gen

if gen $>1$

$\%$ If it is not the first generation, the children from the last

$\%$ generation become the next generation 


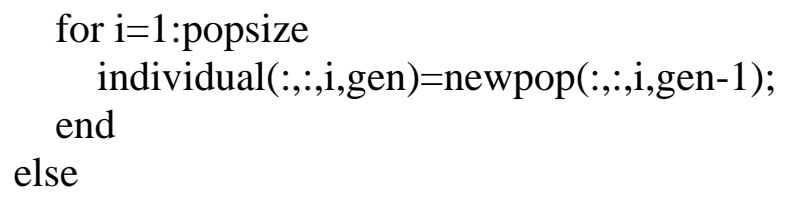

$\%$ The first generation of individuals is randomly generated.

$\%$ The first 5 individuals can be preselected

$\begin{array}{ll}\% & \text { individual(:,:,1,1)=best; } \\ \% & \text { individual(:,:,2,1)=best2; } \\ \% & \text { individual(:,:,2,1)=best3; } \\ \% & \text { individual(:,:,2,1)=best4; } \\ \% & \text { individual(:,:,2,1)=best5; }\end{array}$

for $\mathrm{i}=$ numnotran+1:popsize

\% Each individual is evaluated

for $\mathrm{i}=1$ :popsize

[velocity(i,gen), vel(i,gen)]=GOLF3DGOPT(individual(:,:,i,gen));

end

$\%$ Finding the total fitness of the population

totalfit(gen $)=\operatorname{sum}($ velocity $($,gen $))$;

$\%$ Finding the probability of selection for each chromosome

relfit(:,gen) = velocity(:,gen)/totalfit(gen);

cumfit(:,gen $)=$ cumsum(relfit(:,gen $))$;

\% Keeping track of the most fit individual for all generations

[r(gen),c(gen)] = max(velocity(:,gen));

vel(c(gen),gen)

r(gen)

\% Selecting the top chromosome to remain in the next generation [a,b] = sort(velocity (:,gen));

newpop(:,:,1,gen $)$ = individual(:,:,b(popsize),gen);

\% newpop(:,:,2,gen) = individual(:,:,b(popsize-1),gen); 
\% newpop(:,:,3,gen) = individual(:,:,b(popsize-2),gen);

\% Selecting the chromosomes that will beget the next generation

$\mathrm{x}=[\mathrm{]}$;

for $i=2$ :popsize

$$
\mathrm{x}(\mathrm{i})=\operatorname{rand}(1) \text {; }
$$

for $\mathrm{j}=2$ :popsize

if $\mathrm{x}(\mathrm{i})<\operatorname{cumfit}(1$,gen $)$

parents(:,:,i,gen) = individual(:,:,1,gen);

elseif $x(\mathrm{i})<=$ cumfit(j,gen) \& $\mathrm{x}(\mathrm{i})>=$ cumfit(j-1,gen $)$

parents(:,:,i,gen $)=$ individual(:,:,j,gen);

end

end

end

\% Mutation of chromosomes to develop next generation

$\%$ Mutation occurs by changing the gene (time) by some random amount

for $\mathrm{i}=2$ :popsize

if gen $<$ maxgens $/ 3$

newpop(:,:,i,gen $)=\operatorname{parents}(:,:, i, g e n)+m 1 * \operatorname{randint}(1,4,[0,3]) . * \operatorname{randsrc}(1,4)$;

\% Changing the mutation parameter for the 2nd third of the

$\% \quad$ generations so that smaller adjustments are made.

elseif (gen $>=$ maxgens/3) \& (gen $<$ maxgens $/ 1.5$ )

newpop $(:$, :,i,gen $)=$ parents(:,:,i,gen $)+m 2 *$ randint $(1,4,[0,3]) .{ }^{*} \operatorname{randsrc}(1,4)$;

$\% \quad$ Changing the mutation parameter for the final third of the

$\% \quad$ generations so that smaller adjustments are made.

else

newpop(:,:,i,gen $)=\operatorname{parents}(:$, ,i,gen $)+m 3 * \operatorname{randint}(1,4,[0,3]) . * \operatorname{randsrc}(1,4)$; end

end

end

[maxfit,index]=max(r);

maxfit

best=individual(:,:,c(index),index)

save ('C: Program Files $\backslash$ Autolev $\backslash 3 D G O l F \backslash$ best.txt', 'best', '-ascii', '-tabs');

toc; \% Determines the CPU time to complete the optimization

total_minutes=toc/60 


\section{APPENDIX E}

FORTRAN $^{\odot}$ Code: 3DGOLFG.FOR 
The following code is intended to provide the reader with the general flow of the program and is void of any actual equations and calculations. Due to the length of the $3 \mathrm{D}$ equations, it was not at all feasible to include them within this appendix.

C** The name of this program is 3DGOLFG.FOR

C** Created by AUTOLEV 3.2 on Sun Apr 03 17:11:21 2005

C** Modified by Sasho Mackenzie

$\begin{array}{lc}\text { IMPLICIT } & \text { DOUBLE PRECISION (A - Z) } \\ \text { INTEGER } & \text { ILOOP, IPRINT, PRINTINT } \\ \text { CHARACTER } & \text { MESSAGE(99) } \\ \text { EXTERNAL } & \text { EQNS1,EQNS2,EQNS3,EQNS4,EQNS5,EQNS6 } \\ \text { DIMENSION } & \text { VAR(32) }\end{array}$

LIST OF CONSTANTS, VARIABLES, AND PARAMETERS

C** Open input and output files

C** Read message from input file

C** Read values of constants from input file

C** Read the initial value of each variable from input file

C** Read integration parameters from input file

C** Write heading(s) to output file(s)

C** Degree to radian conversion

C** Initialize time, print counter, variables array for integrator

$\mathrm{T}=$ TINITIAL

IPRINT $=0$

C READING IN THE MOST RECENT OPTIMIZED MUSCLE TIMES FROM MATLAB

C** Initalize numerical integrator with call to EQNS5 at T=TINITIAL

CALL KUTTA(EQNS5, 29, VAR, T, INTEGSTP, ABSERR, RELERR, 0, *5920)

C** Numerically integrate; print results

5900 IF( TFINAL.GE.TINITIAL .AND. T+.01D0*INTEGSTP.GE.TFINAL) IPRINT=-7 IF( TFINAL.LE.TINITIAL .AND. T+.01D0*INTEGSTP.LE.TFINAL) IPRINT=-7 IF( IPRINT .LE. 0 ) THEN 


\author{
CALL IO(T) \\ IF( IPRINT .EQ. -7 ) GOTO 5930 \\ IPRINT $=$ PRINTINT \\ ENDIF
}

IF (SW .EQ. 1) THEN

CALL KUTTA(EQNS1, 32, VAR, T, INTEGSTP, ABSERR, RELERR, 1, *5920) ELSEIF (SW .EQ. 4) THEN

CALL KUTTA(EQNS4, 30, VAR, T, INTEGSTP, ABSERR, RELERR, 1, *5920) ELSEIF (SW .EQ. 3) THEN

CALL KUTTA(EQNS3, 29, VAR, T, INTEGSTP, ABSERR, RELERR, 1, *5920) ELSEIF (SW .EQ. 5) THEN

CALL KUTTA(EQNS5, 29, VAR, T, INTEGSTP, ABSERR, RELERR, 1, *5920) ELSEIF (SW .EQ. 6 .OR. SW .EQ. 7) THEN

CALL KUTTA(EQNS6, 30, VAR, T, INTEGSTP, ABSERR, RELERR, 1, *5920) ELSEIF (SW .EQ. 2) THEN

CALL KUTTA(EQNS2, 28, VAR, T, INTEGSTP, ABSERR, RELERR, 1, *5920) ENDIF

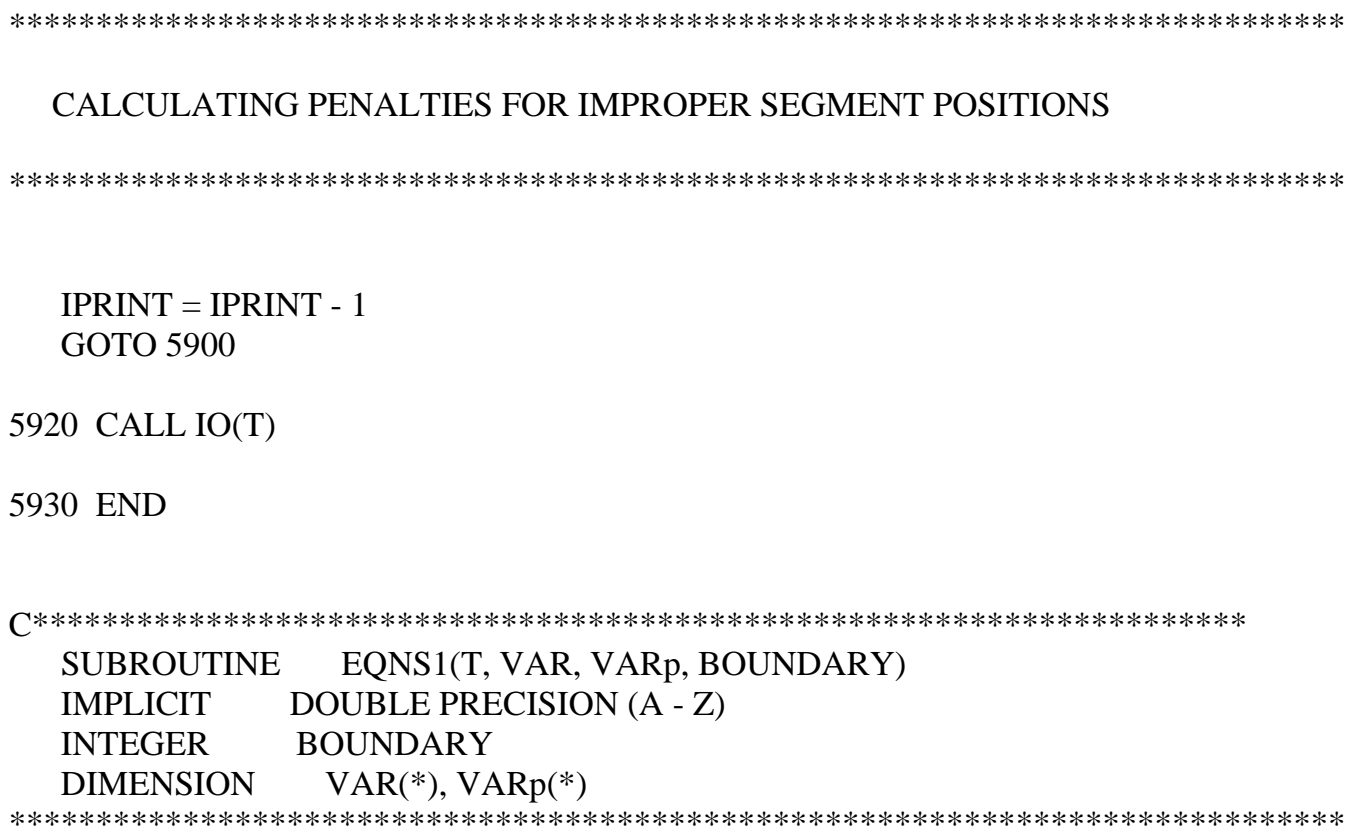

LIST OF CONSTANTS, VARIABLES, AND PARAMETERS 


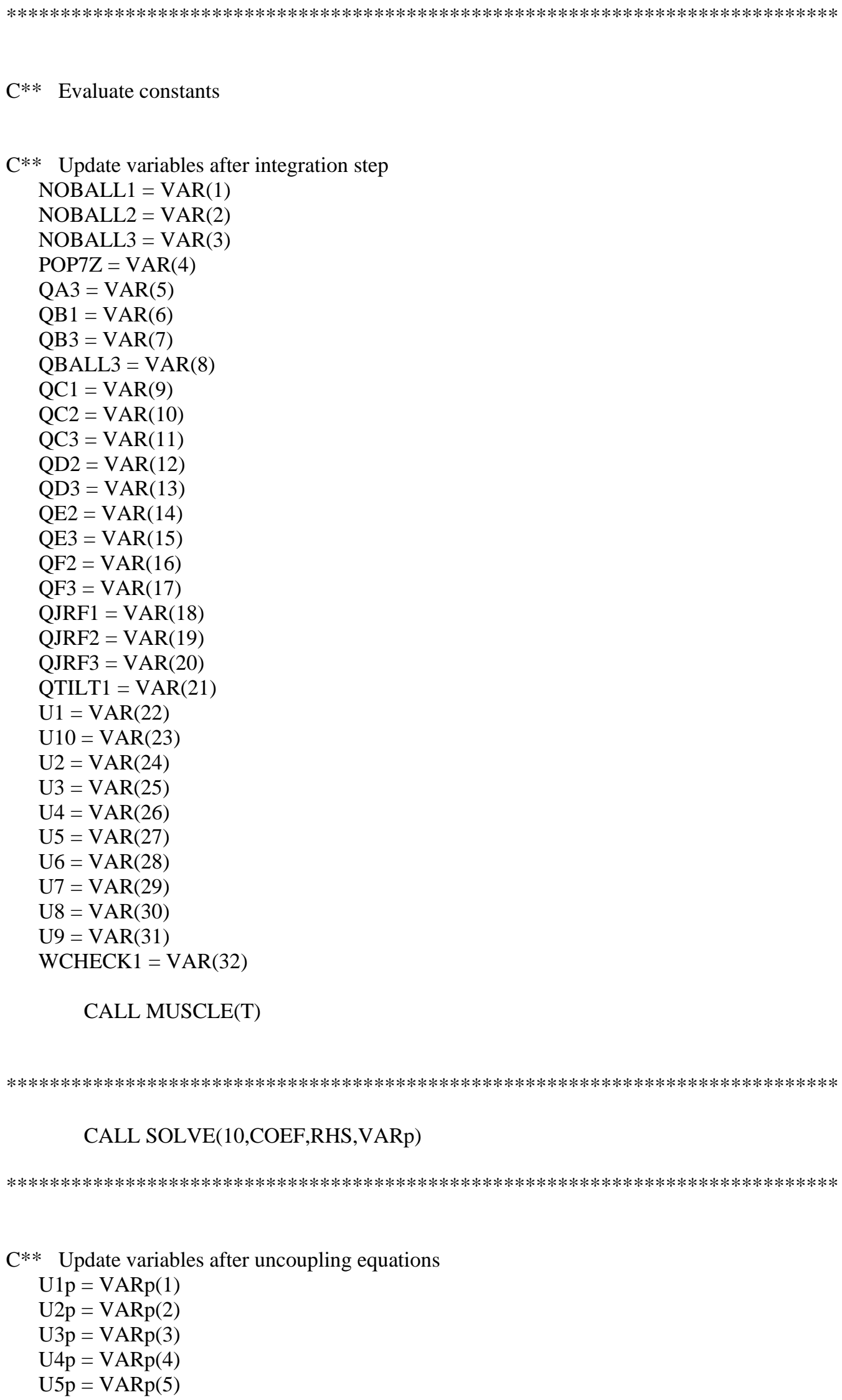

CALL MUSCLE(T)

CALL SOLVE(10,COEF,RHS,VARp)

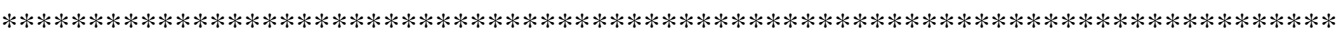

C** Update variables after uncoupling equations

$\mathrm{U} 1 \mathrm{p}=\operatorname{VARp}(1)$

$\mathrm{U} 2 \mathrm{p}=\operatorname{VARp}(2)$

$\mathrm{U} 3 \mathrm{p}=\operatorname{VARp}(3)$

$\mathrm{U} 4 \mathrm{p}=\operatorname{VARp}(4)$

$\mathrm{U} 5 \mathrm{p}=\operatorname{VAR}(5)$ 


$$
\begin{aligned}
& \text { U6p }=\text { VARp(6) } \\
& \text { U7p }=\text { VARp(7) } \\
& \text { U8p }=\text { VARp(8) } \\
& \text { U9p }=\text { VARp(9) } \\
& \text { U10p }=\text { VARp(10) }
\end{aligned}
$$

$C^{* *}$ Update derivative array prior to integration step

VARp(1) $=$ NOBALL1p

$\operatorname{VARp}(2)=$ NOBALL2p

$\operatorname{VARp}(3)=$ NOBALL3p

$\operatorname{VARp}(4)=$ POP7Zp

$\operatorname{VARp}(5)=\mathrm{QA3p}$

$\operatorname{VARp}(6)=\mathrm{QB} 1 \mathrm{p}$

$\operatorname{VARp}(7)=\mathrm{QB} 3 \mathrm{p}$

$\operatorname{VARp}(8)=$ QBALL3p

$\operatorname{VARp}(9)=\mathrm{QC} 1 \mathrm{p}$

$\operatorname{VARp}(10)=\mathrm{QC} 2 \mathrm{p}$

$\operatorname{VARp}(11)=$ QC3p

$\operatorname{VARp}(12)=$ QD2p

$\operatorname{VARp}(13)=\mathrm{QD} 3 \mathrm{p}$

$\operatorname{VARp}(14)=$ QE2p

$\operatorname{VARp}(15)=$ QE3p

$\operatorname{VARp}(16)=\mathrm{QF} 2 \mathrm{p}$

$\operatorname{VARp}(17)=$ QF3p

$\operatorname{VARp}(18)=$ QJRF1p

$\operatorname{VARp}(19)=$ QJRF2p

$\operatorname{VARp}(20)=$ QJRF3p

$\operatorname{VARp}(21)=\mathrm{QTILT} 1 \mathrm{p}$

$\operatorname{VARp}(22)=\mathrm{U} 1 \mathrm{p}$

$\operatorname{VARp}(23)=\mathrm{U} 10 \mathrm{p}$

$\operatorname{VARp}(24)=U 2 \mathrm{p}$

$\operatorname{VARp}(25)=\mathrm{U3p}$

$\operatorname{VARp}(26)=U 4 \mathrm{p}$

$\operatorname{VARp}(27)=$ U5p

$\operatorname{VARp}(28)=\mathrm{U} 6 \mathrm{p}$

$\operatorname{VARp}(29)=U 7 p$

$\operatorname{VARp}(30)=\mathrm{U} 8 \mathrm{p}$

$\operatorname{VARp}(31)=\mathrm{U9p}$

VARp(32) = WCHECK1p

$C^{* *}$ Evaluate output quantities

RETURN

END

$\mathrm{C} * * * * * * * * * * * * * * * * * * * * * * * * * * * * * * * * * * * * * * * * * * * * * * * * * * * * * * * * * * * * * * * * * * * * * * *$

C START OF EQNS2: SHOULDER, ARM, AND WRIST CONSTRAINED

\footnotetext{
$\mathrm{C} * * * * * * * * * * * * * * * * * * * * * * * * * * * * * * * * * * * * * * * * * * * * * * * * * * * * * * * * * * * * * * * * * * * * * *$

SUBROUTINE EQNS2(T, VAR, VARp, BOUNDARY)

IMPLICIT DOUBLE PRECISION (A - Z)

INTEGER BOUNDARY

DIMENSION $\operatorname{VAR}(*), \operatorname{VARp}(*)$
} 
C** Update variables after integration step

CALL MUSCLE(T)

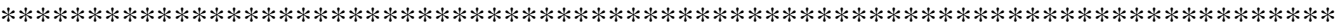

CALL SOLVE(7,COEF,RHS,VARp)

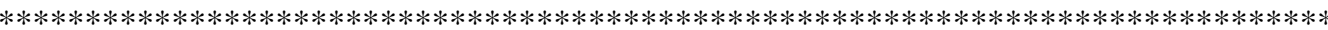

C** Update variables after uncoupling equations

C** Update derivative array prior to integration step

C** Evaluate output quantities

RETURN

END

$\mathrm{C} * * * * * * * * * * * * * * * * * * * * * * * * * * * * * * * * * * * * * * * * * * * * * * * * * * * * * * * * * * * * * * * * * * * * * * *$

C START OF EQNS3: ARM LONGITUDINAL ROTATION AND WRIST CONSTRAINED

$\mathrm{C} * * * * * * * * * * * * * * * * * * * * * * * * * * * * * * * * * * * * * * * * * * * * * * * * * * * * * * * * * * * * * * * * * * * * * * * * * * *)$

SUBROUTINE EQNS3(T, VAR, VARp, BOUNDARY)

IMPLICIT DOUBLE PRECISION (A - Z)

INTEGER BOUNDARY

DIMENSION $\operatorname{VAR}(*), \operatorname{VARp}(*)$

LIST OF CONSTANTS, VARIABLES, AND PARAMETERS

C** Evaluate constants

C** Update variables after integration step 
CALL MUSCLE(T)

\section{CALL SOLVE(8,COEF,RHS,VARp)}

C** Update variables after uncoupling equations

$C^{* *}$ Update derivative array prior to integration step

C** Evaluate output quantities

RETURN

END

$\mathrm{C} * * * * * * * * * * * * * * * * * * * * * * * * * * * * * * * * * * * * * * * * * * * * * * * * * * * * * * * * * * * * * * * * * * * * * * *$

C START OF EQNS4: ARM LONGITUDINAL ROTATION CONSTRAINED

$\mathrm{C} * * * * * * * * * * * * * * * * * * * * * * * * * * * * * * * * * * * * * * * * * * * * * * * * * * * * * * * * * * * * * * * * * * * * * * * * *$

SUBROUTINE EQNS4(T, VAR, VARp, BOUNDARY)

IMPLICIT DOUBLE PRECISION (A - Z)

INTEGER BOUNDARY

DIMENSION $\operatorname{VAR}(*), \operatorname{VARp}(*)$

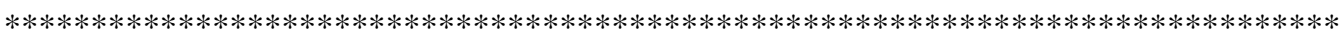

LIST OF CONSTANTS, VARIABLES, AND PARAMETERS

C** Evaluate constants

C** Update variables after integration step

CALL MUSCLE(T)

$* * * * * * * * * * * * * * * * * * * * * * * * * * * * * * * * * * * * * * * * * * * * * * * * * * * * * * * * * * * * * * * * * * * * * * * * * * * * *$

CALL SOLVE(9,COEF,RHS,VARp)

C** Update variables after uncoupling equations

C** Update derivative array prior to integration step

C** Evaluate output quantities 


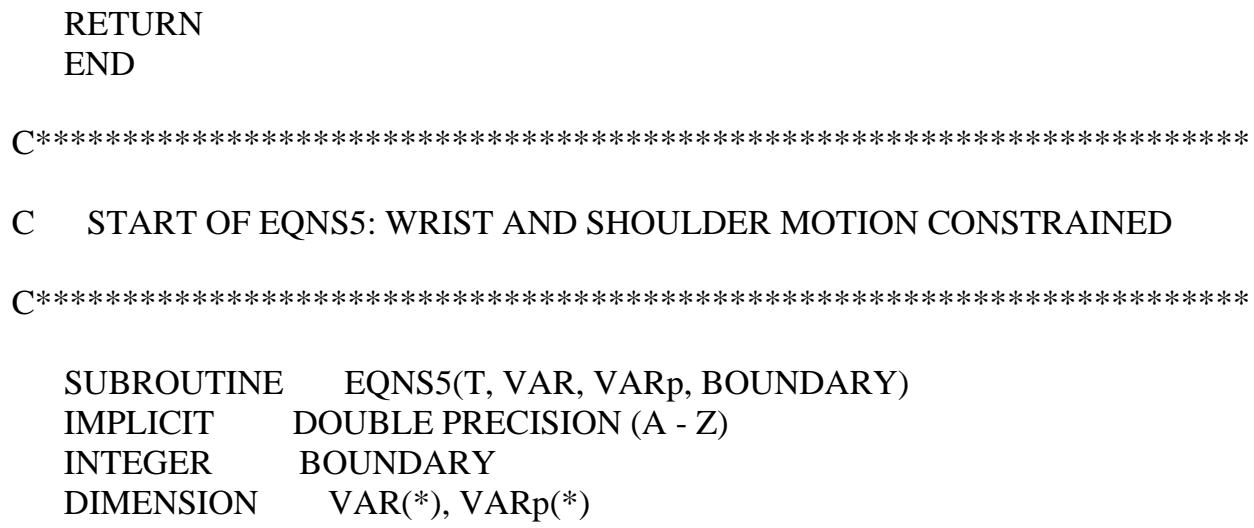

\section{LIST OF CONSTANTS, VARIABLES, AND PARAMETERS}

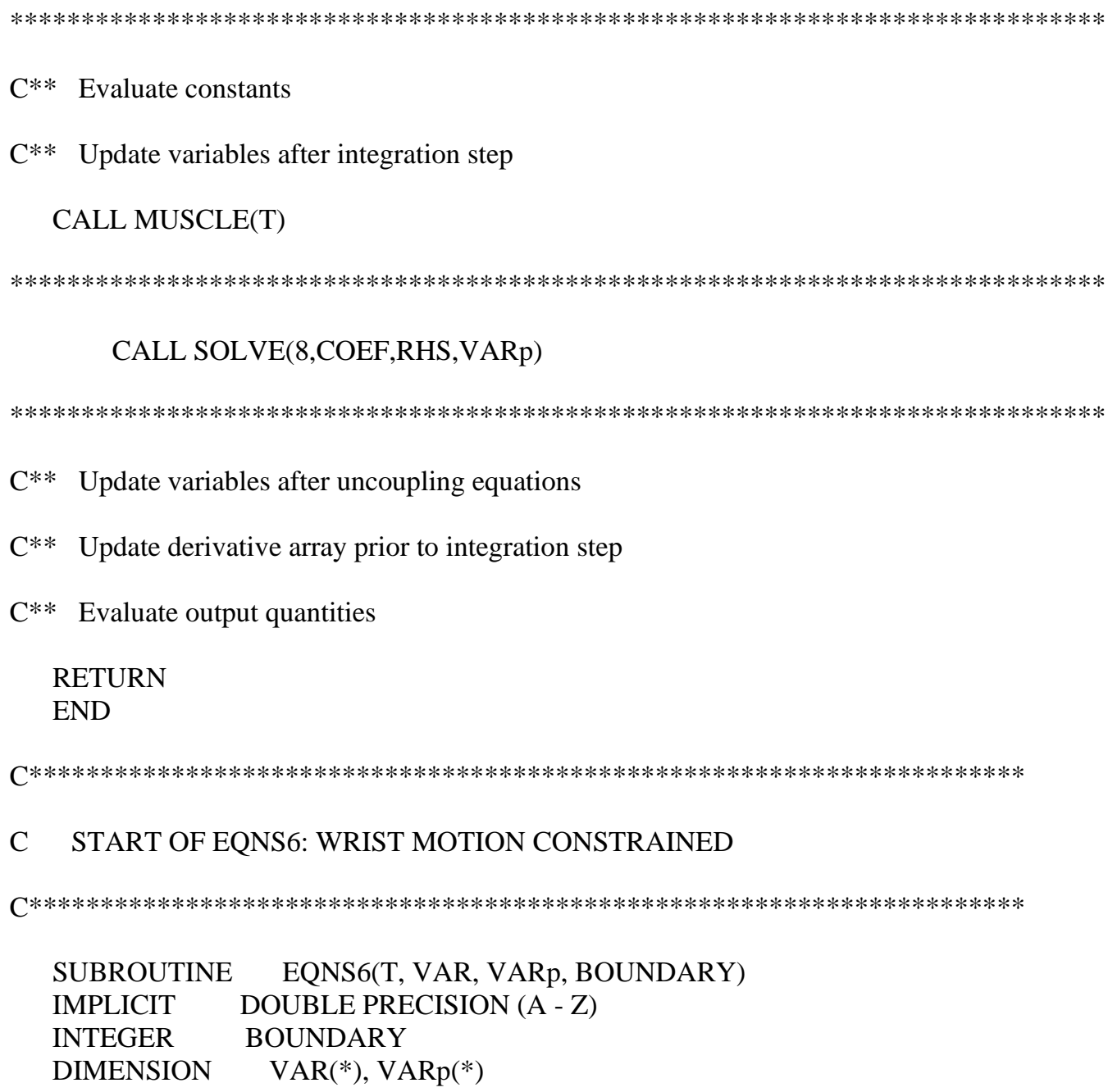




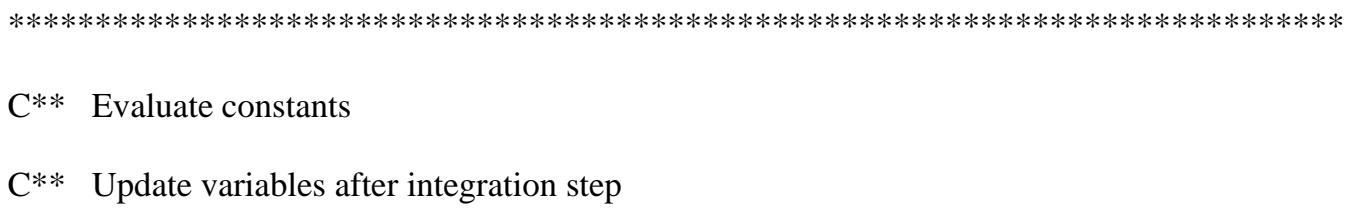

CALL MUSCLE(T)

\section{CALL SOLVE(9,COEF,RHS,VARp)}

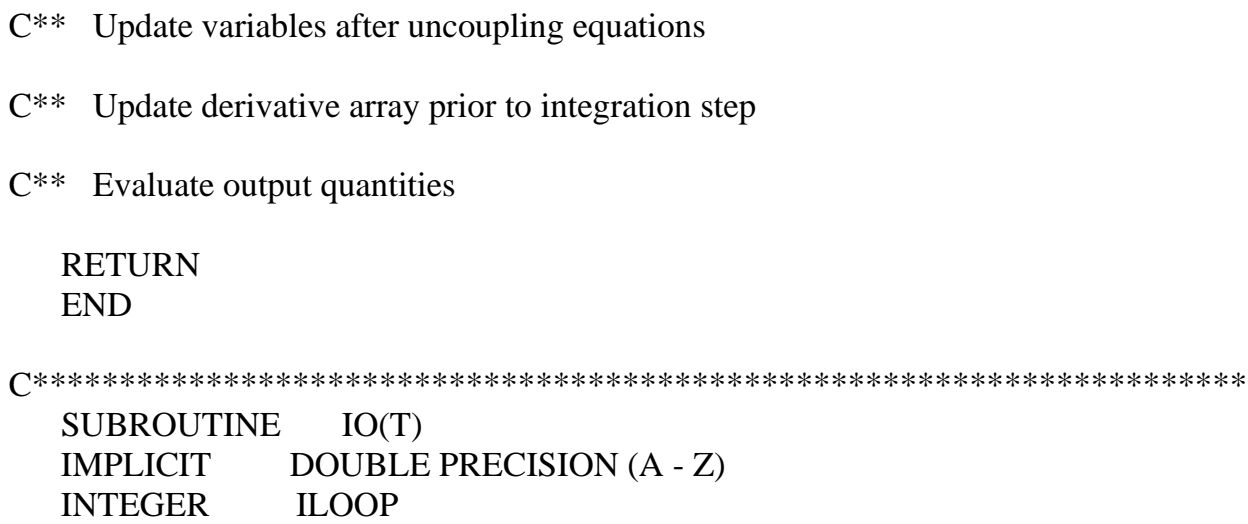

LIST OF CONSTANTS, VARIABLES, AND PARAMETERS

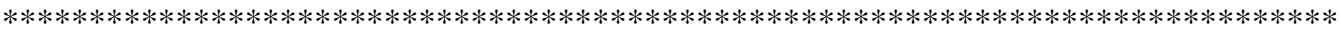

\section{WRITING DATA TO OUTPUT FILES}

RETURN

END

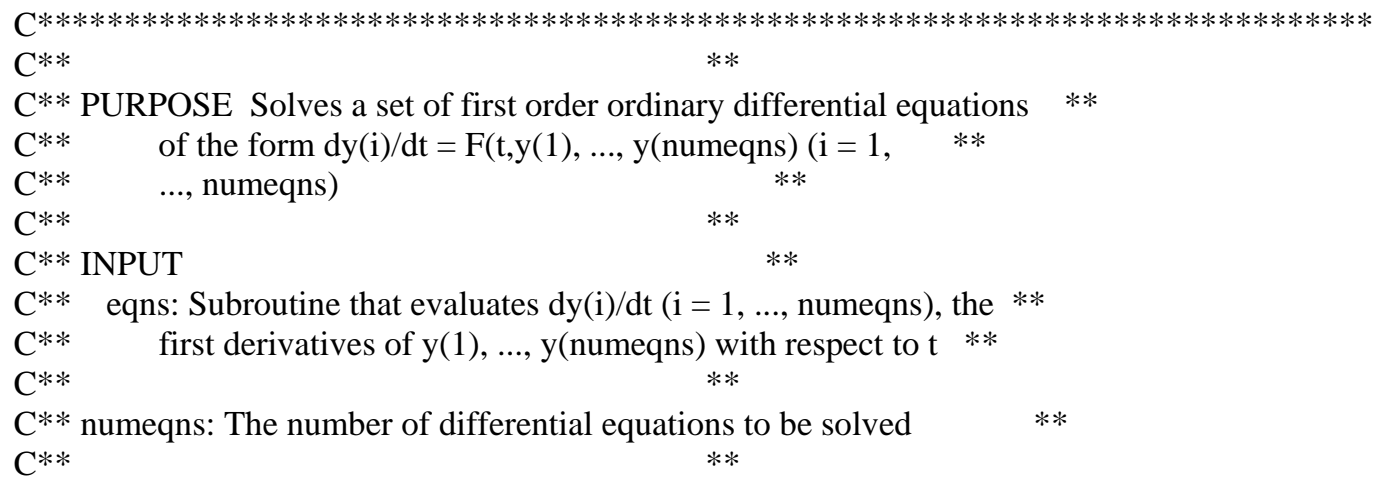




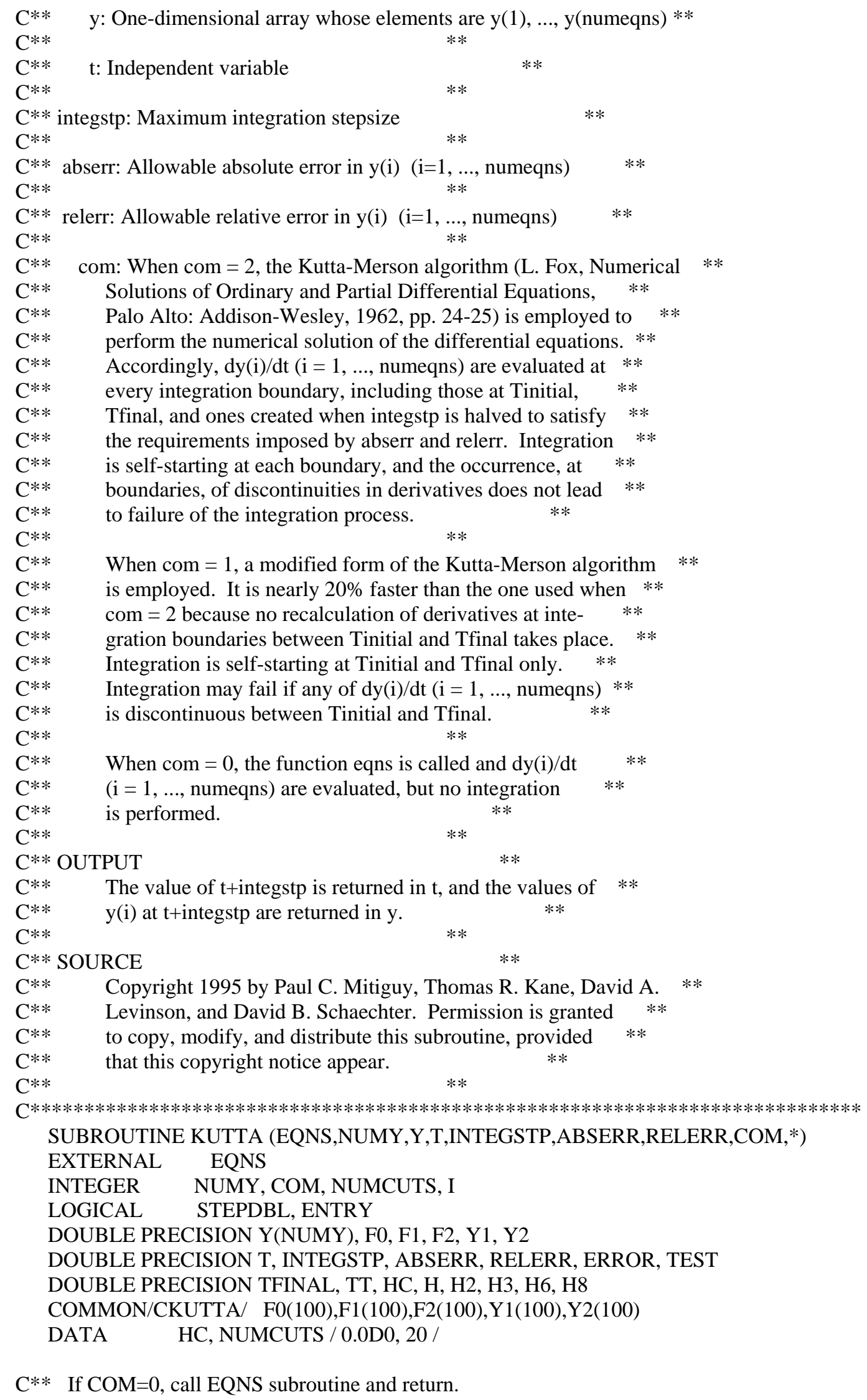




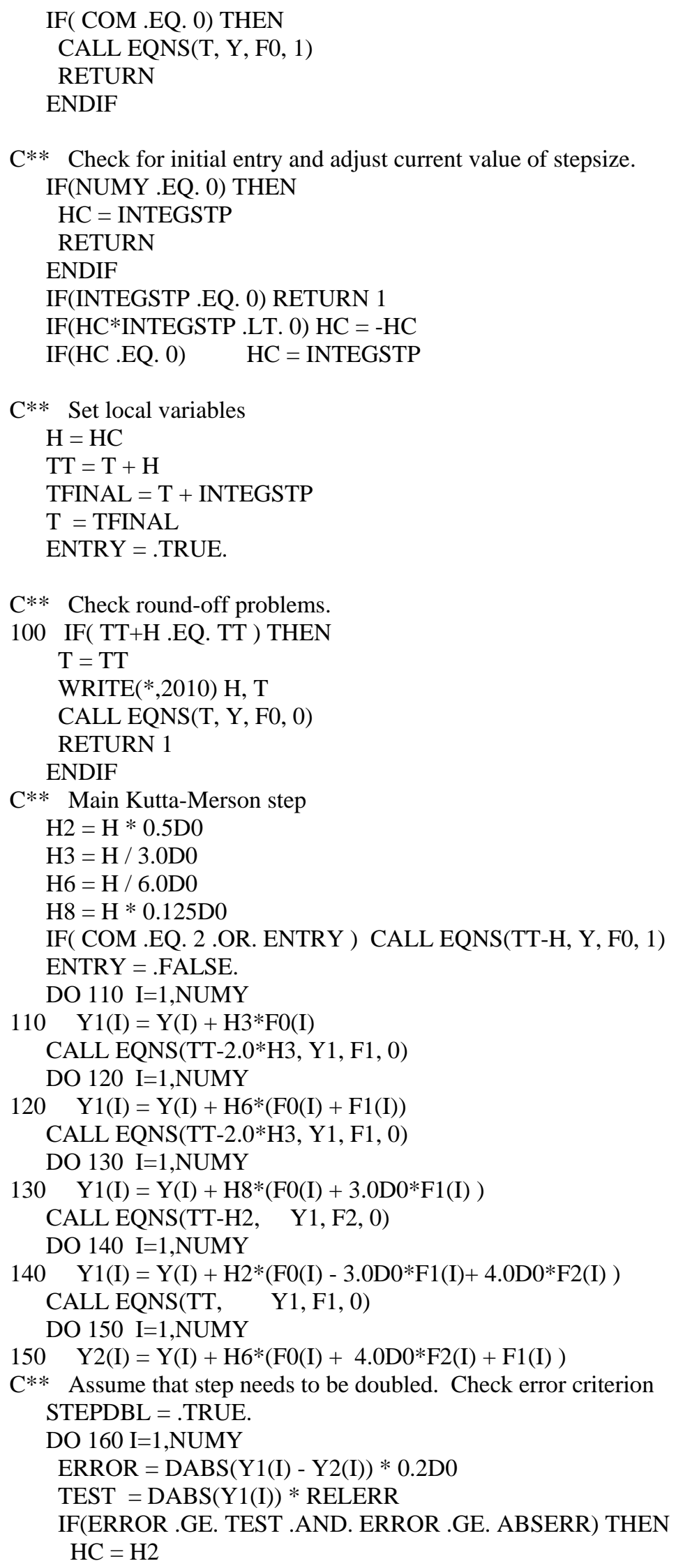




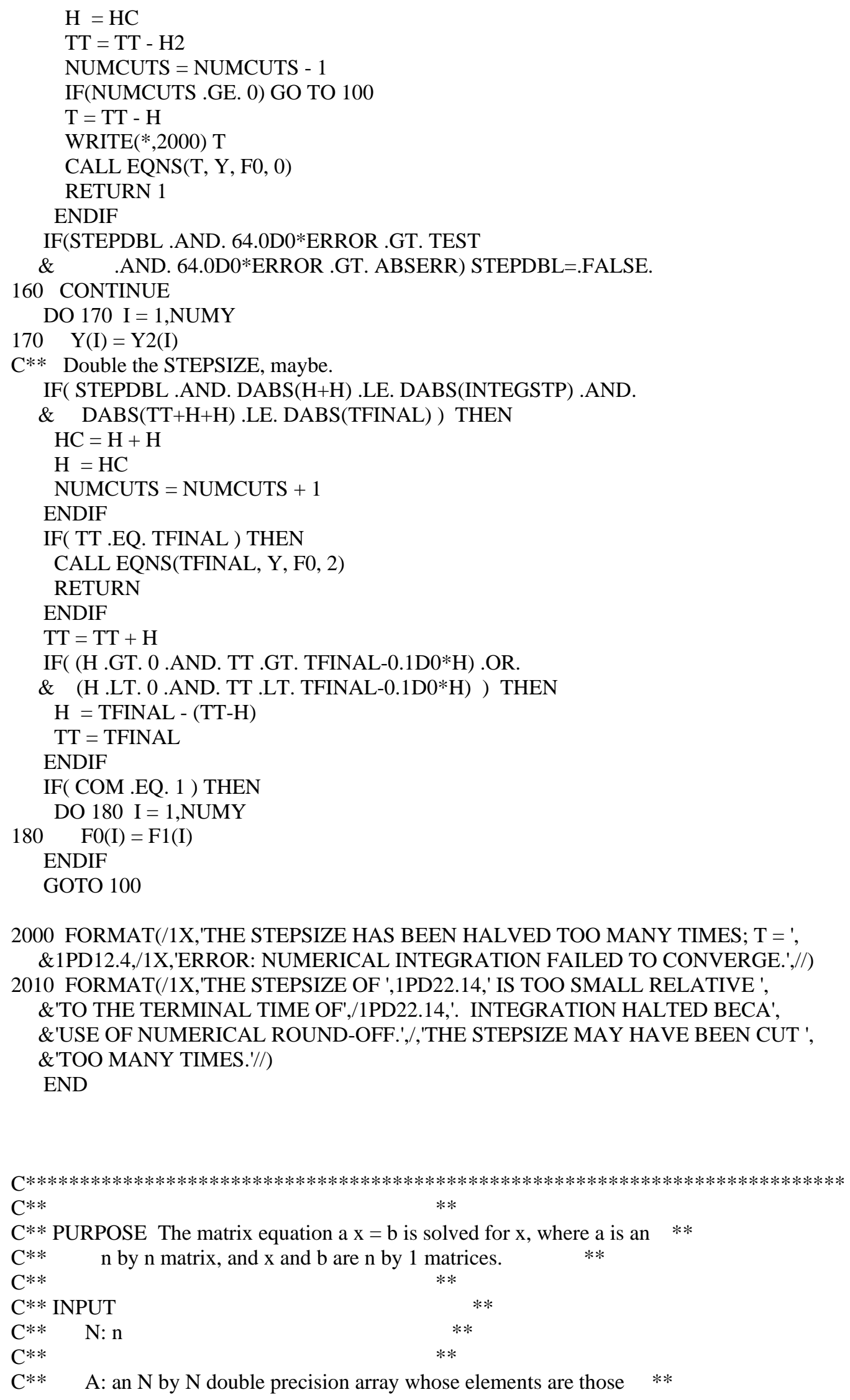




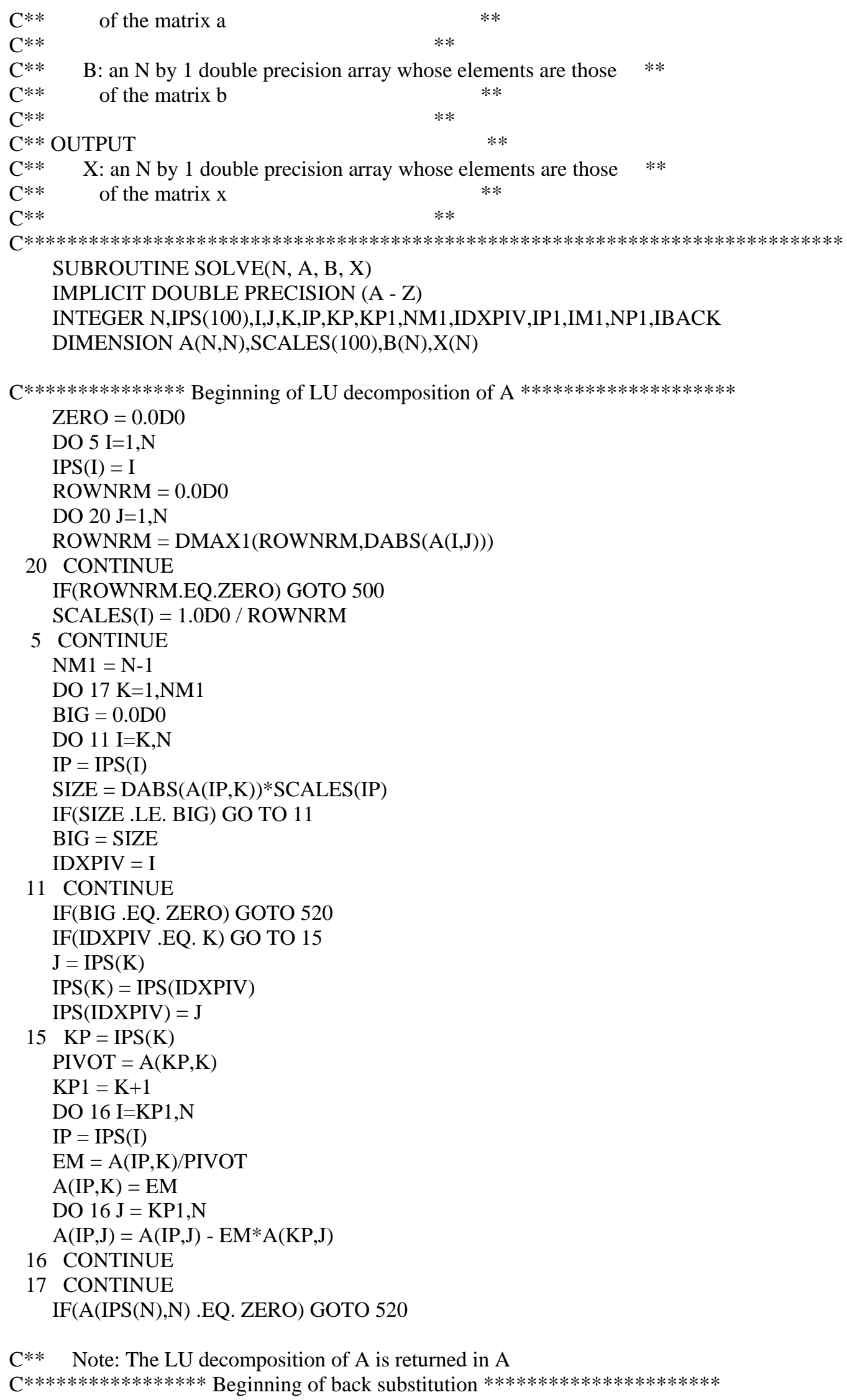




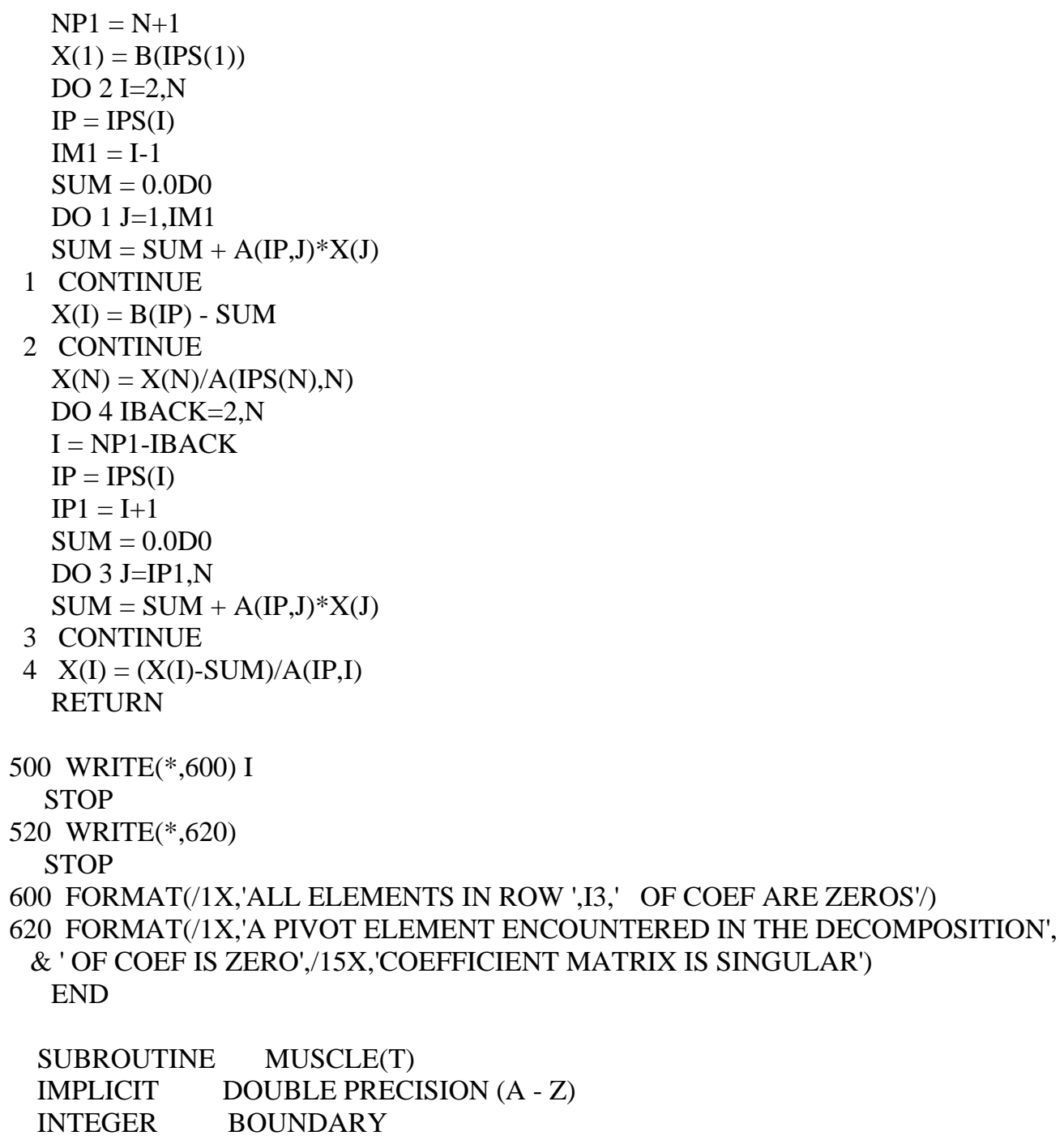

LIST OF CONSTANTS, VARIABLES, AND PARAMETERS

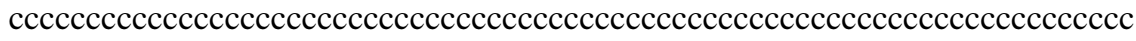

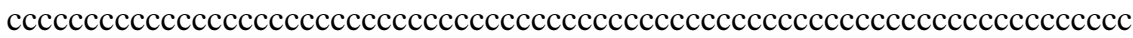

c START OF MUSCLE MODEL EQUATIONS

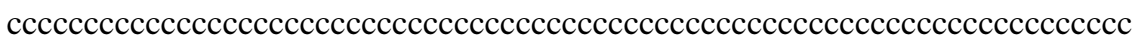

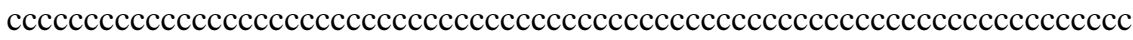

pinc $=1.0 !$ The percentage increase in the maximum torque values

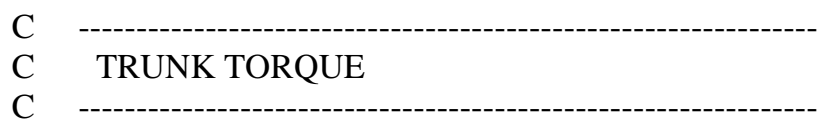




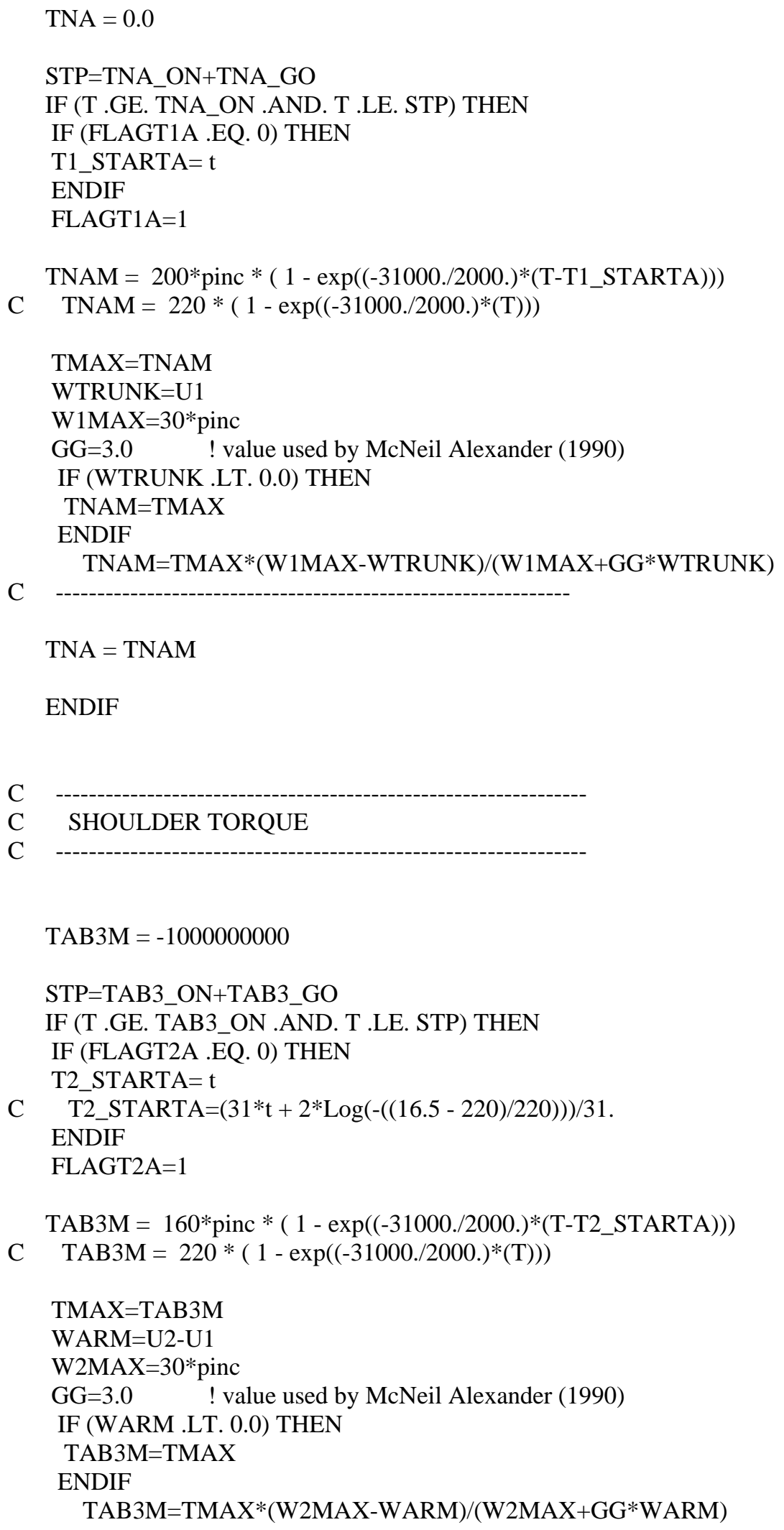


C $\quad$ TAB3 $=$ TAB3M

ENDIF

C open( 8, FILE='ANGLES.DAT',

C * STATUS='UNKNOWN', ACCESS='APPEND' )

C write( 8, '(4(f16.4,3X))' ) T,SW,TAB3M,TAB3

C close(8)

IF (SW .EQ. 2 .OR. SW .EQ. 5) THEN

IF (TAB3M .GE. TAB3) SW=6

ENDIF

$\mathrm{TAB} 3=\mathrm{TAB} 3 \mathrm{M}$

IF (T .GT. STP) TAB $3=0.0$

IF (WARM .GT. W2MAX) TAB3 $=0$

IF (TAB3 .EQ. -1000000000) TAB3=0 ! PROBLEM IS HERE!!!!!!!!!

C

C WRIST TORQUE

C

$\mathrm{TBC} 3 \mathrm{M}=0$

STP=TBC3_ON+TBC3_GO

IF (T .GE. TBC3_ON .AND. T .LE. STP) THEN

IF (FLAGT3A .EQ. 0) THEN

T3_STARTA $=\mathrm{t}$

ENDIF

FLAGT3A=1

TBC3M $=90 *$ pinc $*\left(1-\exp \left((-31000 . / 2000) *.\left(T-T 3 \_S T A R T A\right)\right)\right)$

C $\quad$ TBC3M $=220 *(1-\exp ((-31000 . / 2000) *.(T)))$

TMAX $=$ TBC3M

WCLUB $=\mathrm{U} 4$

W3MAX $=60 *$ pinc

$\mathrm{GG}=3.0$ ! value used by McNeil Alexander (1990)

IF (WCLUB .LT. 0.0) THEN

TBC3M=TMAX

ENDIF

TBC3M=TMAX*(W3MAX-WCLUB)/(W3MAX+GG*WCLUB)

C

C $\quad$ TBC3 $=$ TBC3M

ENDIF

IF (SW .GT. 1 .AND. SW .LT. 7) THEN

IF (TBC3M .GT. TBC3 .AND. T .GT. .1) SW=1 ! set $\mathrm{SW}=1$ for if no tab1 torque ENDIF

TBC3 $=$ TBC3M

IF (WCLUB .GT. W3MAX) TBC3=0

IF (T .GT. STP) TBC3 $=0.0$

c $\quad$ IF ( (QC3*57.3).GT. -20$)$ TBC3 $=-20.0$

C $\quad \operatorname{IF}((\mathrm{QC} 3 * 57.3) . \mathrm{GT} .-10) \mathrm{SW}=6$ 


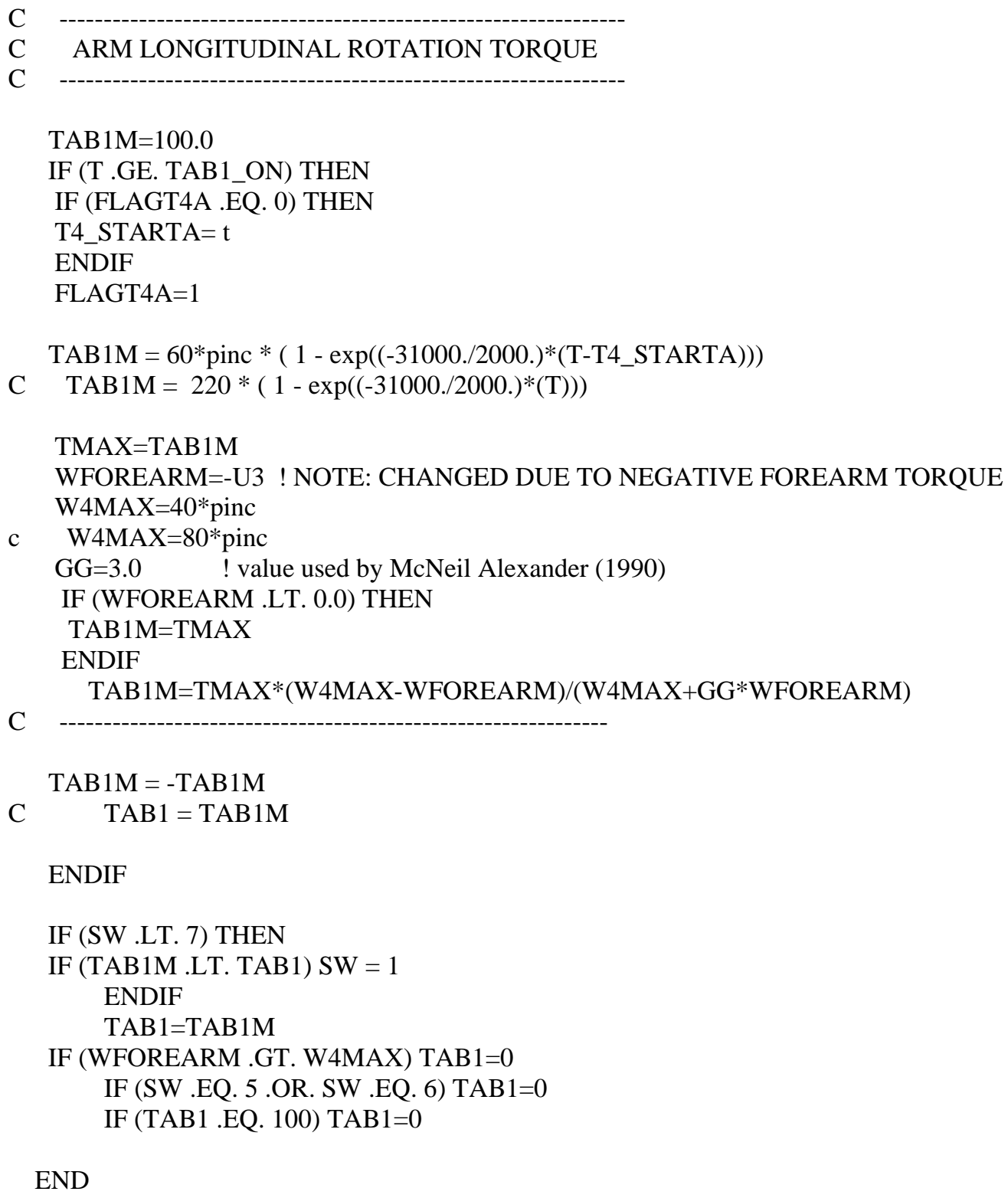

